\title{
ABSTRACT
}

\section{SEARCH FOR THE STANDARD MODEL HIGGS BOSON IN THE FOUR LEPTON FINAL STATE BY THE DO EXPERIMENT AT RUN II OF THE TEVATRON COLLIDER}

\author{
Diego Menezes, Ph.D. \\ Department of Physics \\ Northern Illinois University, 2013 \\ David Hedin, Director
}

This dissertation presents a measurement of $Z$ boson pair production in $p \bar{p}$ collisions at 1.96 $\mathrm{TeV}$ with $9.6 \mathrm{fb}^{-1}$ to $9.8 \mathrm{fb}^{-1}$ of $\mathrm{D} 0$ data. We examine the final states eeee, ee $\mu \mu$, and $\mu \mu \mu \mu$. Based on selected data, the measured cross section in the mass region $M\left(Z / \gamma^{*}\right)>30 \mathrm{GeV}$ is $\sigma\left(p \bar{p} \rightarrow Z / \gamma^{*} Z / \gamma^{*}\right)=1.26_{-0.36}^{+0.44}$ (stat) ${ }_{-0.15}^{+0.17}$ (syst) \pm 0.08 (lumi) pb. After correcting for the expected ratio of $\sigma\left(p \bar{p} \rightarrow Z / \gamma^{*} Z / \gamma^{*}\right)$ to $\sigma(p \bar{p} \rightarrow Z Z)$, we derive a cross section for $p \bar{p} \rightarrow Z Z$ production of $1.05_{-0.30}^{+0.37}$ (stat) $)_{-0.12}^{+0.14}$ (syst) \pm 0.06 (lumi) pb. This result is combined with a previous result from the $Z Z \rightarrow \ell^{+} \ell^{-} \nu \bar{\nu}$ channel resulting in a combined $p \bar{p} \rightarrow Z Z$ cross section measurement of $1.32_{-0.25}^{+0.29}$ (stat) \pm 0.12 (syst) \pm 0.04 (lumi) pb. These measurements are consistent with the standard model expectation of $1.43 \pm 0.10 \mathrm{pb}$. We extend this analysis to search for the standard model (SM) Higgs boson between 115 and $200 \mathrm{GeV}$. At a Higgs boson mass of $125 \mathrm{GeV}$, we expect to set a limit of 43 times the SM expectation at $95 \%$ C.L., and set a limit of 42 times the SM expectation at 95\% C.L. 


\title{
NORTHERN ILLINOIS UNIVERSITY
}

DE KALB, ILLINOIS

AUGUST 2013

SEARCH FOR THE STANDARD MODEL HIGGS BOSON IN THE FOUR LEPTON FINAL STATE BY THE D0 EXPERIMENT AT RUN II OF THE TEVATRON COLLIDER

\author{
BY \\ DIEGO MENEZES \\ 2013 (C) Diego Menezes \\ DEPARTMENT OF PHYSICS
}

A DISSERTATION SUBMITTED TO THE GRADUATE SCHOOL IN PARTIAL FULFILLMENT OF THE REQUIREMENTS FOR THE DEGREE DOCTOR OF PHILOSOPHY

Doctoral Director:

David Hedin 


\section{ACKNOWLEDGEMENTS}

I want to express my gratitude to the NICADD physicists whom I had the pleasure to work with for all those years.

I would like to express my immense gratitude to Dr. Sergey Uzunyan. His help goes far beyond any discussion on physics and any other related issue.

I thank all members of $\mathrm{D} \varnothing$ and the Tevatron staff whose efforts greatly contribute to make this work possible. I am especially grateful to members and convenors of the DØ Top group for their help with this analysis, specially James Krauss, Ryan Hooper and Lei Feng.

I thank my colleagues and friends, Castro Abughayada, Fayez Abu-Ajamihe, Mike Latimer, Chris Prokop, Ben Blomberg, Harsha Panuganti, Andrew Gearhart, Anthony Gee, Jon Maloney, Nilanjana Kumar, Preeti Vodnala and many others. My life in this small town would have been impossible without all of you around.

It is a pleasure to express my to gratitude Dr. Suzanne Willis for all fruitfull dicussions and enthusiasm that led to this work. Her help and support go far beyond these pages.

Through these years I always thought that in the end I would not have enough words to describe how much I am grateful to my advisor Dr. David Hedin. I was right! Since the very beginning until this moment, his help, guidance, patience and friendship 
were always there when I needed the most. I will take it all with me no matter where I go from here.

I would like to acknowledge the financial support of the National Science Foundation. 


\section{CONTENTS}

Page

LIST OF TABLES $\ldots \ldots \ldots \ldots \ldots \ldots \ldots \ldots \ldots \ldots \ldots \ldots \ldots \ldots$ viii

LIST OF FIGURES. . . . . . . . . . . . . . . . . . . . . xvi

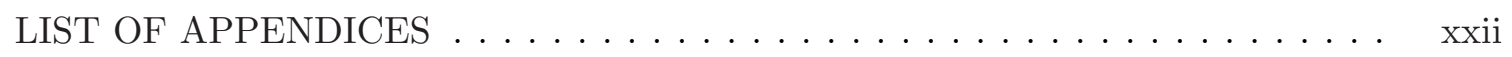

Chapters

1 INTRODUCTION ......................... 1

1.1 The Standard Model of Particle Physics. . . . . . . . . . . . . . . . . 1

1.1.1 The Electroweak Sector. . . . . . . . . . . . . . 3

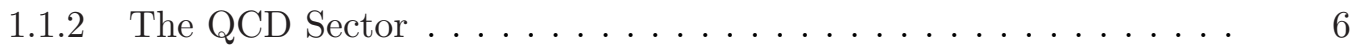

1.1.3 The Standard Model Higgs Mechanism . . . . . . . . . . . . . . 9

1.1.4 The Standard Model Higgs Boson Production . . . . . . . . . . . . . . 13

2 THE TEVATRON COLLIDER AND THE Do DETECTOR . . . . . . . . . . 18

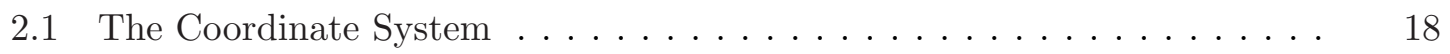

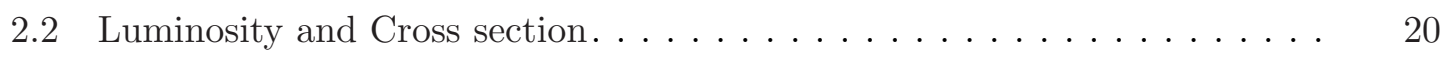

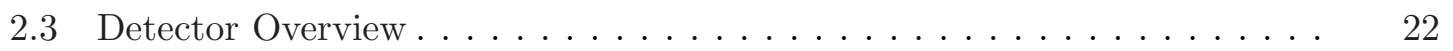

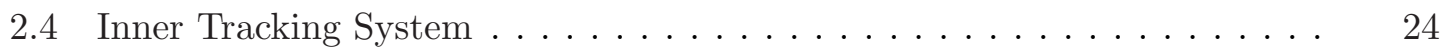

2.4.1 Silicon Microstrip Tracker . . . . . . . . . . . . . 25

2.4 .2 Central Fiber Tracker . . . . . . . . . . . . . . . 26

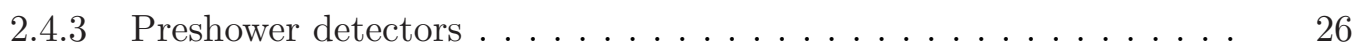




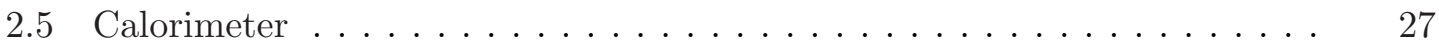

2.5.1 ICD and Massless Gaps. . . . . . . . . . . . . . . . . . . 31

2.6 Luminosity Monitor . . . . . . . . . . . . . . . . . . . . . . . . . . . 31

2.7 The Muon System . . . . . . . . . . . . . . . . . . . . . . 32

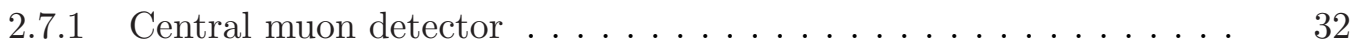

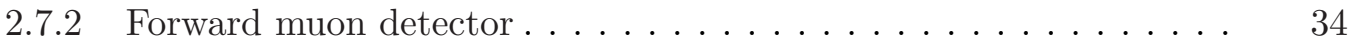

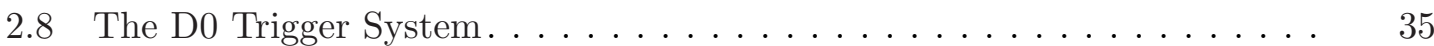

3 DATA AND MONTE CARLO SAMPLES, TRIGGER AND LUMINOSITY . . 37

3.1 Data Samples . . . . . . . . . . . . . . . . . . . . . . . . 37

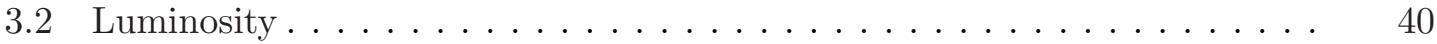

3.3 Monte Carlo Samples . . . . . . . . . . . . . . . . . . . . . . . . . . . . 41

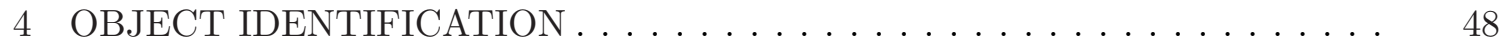

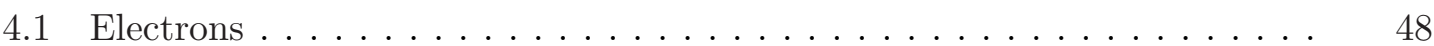

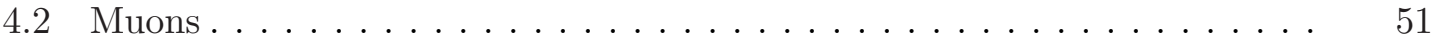

4.3 Missing Energy . . . . . . . . . . . . . . . . . . . . 55

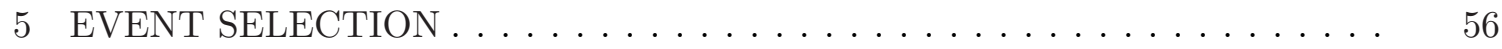

5.1 Signal eeee channel . . . . . . . . . . . . . . . . . . . . . 56

5.2 Signal $\mu \mu \mu \mu$ channel. . . . . . . . . . . . . . . . . . . . . 62

5.2 .1 Muon quality and charge.................. 68

5.3 Signal ee $\mu \mu$ channel . . . . . . . . . . . . . . . . . . . . . . 72

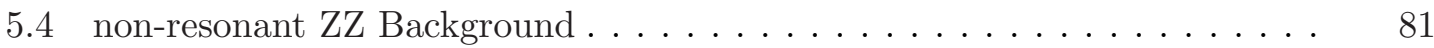

5.4 .1 eeee channel . . . . . . . . . . . . . . . . . . . 81

$5.4 .2 \mu \mu \mu \mu$ channel . . . . . . . . . . . . . . . . . 83

$5.4 .3 e е \mu \mu$ channel. . . . . . . . . . . . . . . . . 84 


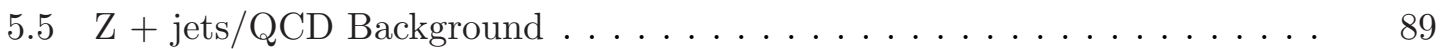

5.5.1 Electron fakerate and $\mathrm{Z}+$ jets background determination in eeee channel . . . . . . . . . . . . . . . . . . . . . . 89

5.5.2 Muon fakerate and Z + jets background determination in $\mu \mu \mu \mu$ channel ................................ 92

$5.5 .3 е е \mu \mu \mathrm{Z}+$ jets Background................. 93

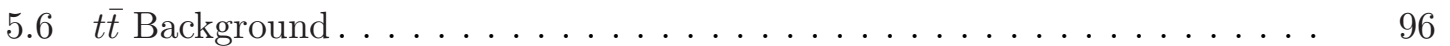

5.7 Beam Halo and Cosmic Ray Muon Background . . . . . . . . . . . . . . . 97

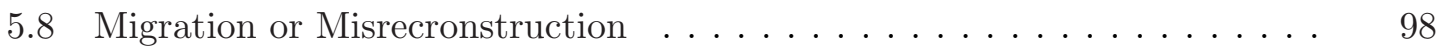

6 SELECTION RESULTS AND CROSS SECTION CALCULATION. . . . . . . 100

7 SYSTEMATICS . . . . . . . . . . . . . . . . . . . . . . . . . . . . . 109

8 LIMITS CALCULATION. . . . . . . . . . . . . . . . . . . . . . . . 125

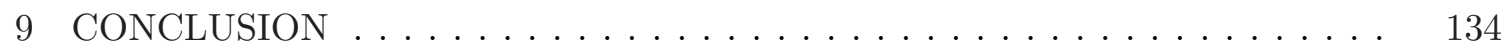

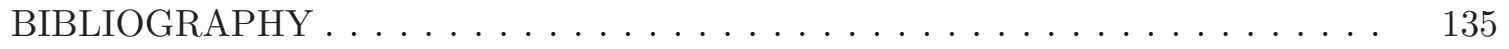

APPENDICES . . . . . . . . . . . . . . . . . . . . . . . . . . 140 


\section{LIST OF TABLES}

Table

Page

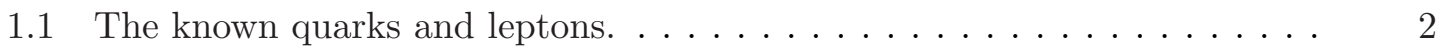

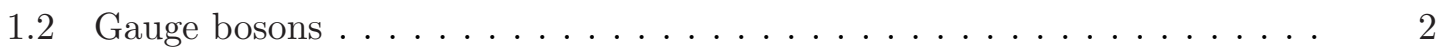

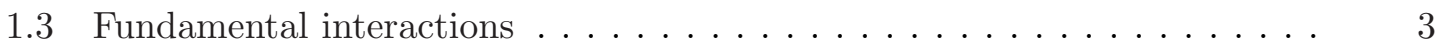

3.1 Table of luminosities used in this analysis. The error is the $6.1 \%$ standard luminosity uncertainty. . . . . . . . . . . . . . . . 41

3.2 RunIIa Pythia Higgs $g g \rightarrow H$ MC samples used in this analysis.. . . . . 43

3.3 RunIIb Pythia Higgs $g g \rightarrow H$ MC samples used in this analysis. . . . . . 44

3.4 RunIIa Pythia Higgs ZH MC samples used in this analysis. . . . . . . 45

3.5 RunIIb Pythia Higgs $Z H$ MC samples used in this analysis. . . . . . . . 46

3.6 RunIIa Pythia GEANT MC samples used in this analysis. . . . . . . . . . 47

3.7 RunIIb Pythia GEANT MC samples used in this analysis. . . . . . . . . . 47

5.1 RunIIa: Cut flow for eeee data for four subchannels $(=2,=3, \geq 4$ central electrons with no ICR electrons, and with 1 ICR electron). . . . . . . . . 59

5.2 RunIIb: Cut flow for eeee data for four subchannels $(=2,=3$ or $\geq 4$ central electrons with no ICR electrons, and with 1 ICR electron). . . . . 59

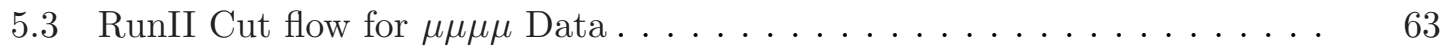


5.4 RunII Cut flow for $H \rightarrow Z Z \rightarrow \mu \mu \mu \mu$ weighted MC signal $\left(m_{H}=125\right.$

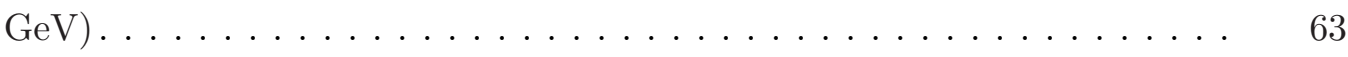

5.5 RunII Cut flow for $Z H \rightarrow \mu \mu \mu \mu$ weighted MC signal $\left(m_{H}=125 \mathrm{GeV}\right) \ldots \quad 65$

5.6 RunII Cut flow for $H \rightarrow Z Z \rightarrow \mu \mu \mu \mu$ weighted MC signal $\left(m_{H}=180\right.$

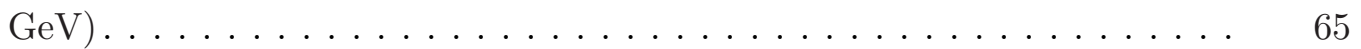

5.7 RunII Cut flow for $Z H \rightarrow \mu \mu \mu \mu$ weighted MC signal $\left(m_{H}=180 \mathrm{GeV}\right) \ldots \quad 66$

5.8 Cut flow for RunII $H \rightarrow Z Z \rightarrow \mu \mu \mu \mu$ MC signal showing cumulative acceptance times efficiency $\left(m_{H}=125 \mathrm{GeV}\right) \ldots \ldots \ldots \ldots 67$

5.9 Cut flow for RunII $Z H \rightarrow \mu \mu \mu \mu \mathrm{MC}$ signal showing cumulative acceptance times efficiency $\left(m_{H}=125 \mathrm{GeV}\right) \ldots \ldots \ldots \ldots \ldots \ldots$

5.10 Cut flow for RunII $H \rightarrow Z Z \rightarrow \mu \mu \mu \mu$ MC signal showing cumulative acceptance times efficiency $\left(m_{H}=180 \mathrm{GeV}\right) \ldots \ldots \ldots \ldots$

5.11 Cut flow for RunII $Z H \rightarrow \mu \mu \mu \mu \mathrm{MC}$ signal showing cumulative acceptance times efficiency $\left(m_{H}=180 \mathrm{GeV}\right) \ldots \ldots \ldots \ldots \ldots \ldots \ldots$

5.12 Charge distribution for RunII $\mu \mu \mu \mu$ data . . . . . . . . . . . 70

5.13 Charge distribution for RunII $\mu \mu \mu \mu \mathrm{MC}$ signal . . . . . . . . . . . . . 70

5.14 nseg distribution for RunII $\mu \mu \mu \mu$ data . . . . . . . . . . . . 71

5.15 The fraction (\%) of muon topology (nseg) distribution for RunII $\mu \mu \mu \mu \mathrm{MC}$

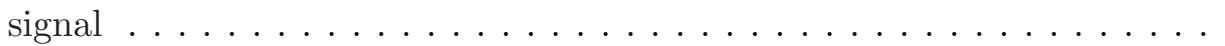

5.16 RunIIa: Cut flow for $е e \mu \mu$ data for three subchannels $(=0,=1$ or $\geq 2$

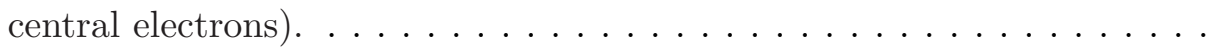

5.17 RunIIb: Cut flow for ee $\mu \mu$ data for three subchannels $(=0,=1$ or $\geq 2$

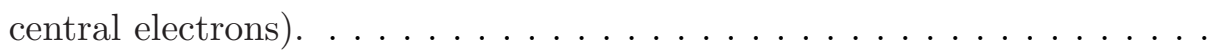


5.18 RunIIa: Cut flow for $H \rightarrow Z Z \rightarrow e e \mu \mu$ MC signal $\left(m_{H}=125 \mathrm{GeV}\right)$ showing cumulative acceptance times efficiency values for three subchannels $(=0,=1$ or $\geq 2$ central electrons). Only statistical errors are shown.

5.19 RunIIa: Cut flow for $Z H \rightarrow e e \mu \mu \mathrm{MC}$ signal $\left(m_{H}=125 \mathrm{GeV}\right)$ showing cumulative acceptance times efficiency values for three subchannels $(=0$, $=1$ or $\geq 2$ central electrons). Only statistical errors are shown. . . . . . .

5.20 RunIIa: Cut flow for $H \rightarrow Z Z \rightarrow e e \mu \mu \mathrm{MC}$ signal $\left(m_{H}=180 \mathrm{GeV}\right)$ showing cumulative acceptance times efficiency values for three subchannels $(=0,=1$ or $\geq 2$ central electrons). Only statistical errors are shown.

75

5.21 RunIIa: Cut flow for $Z H \rightarrow e е \mu \mu \mathrm{MC}$ signal $\left(m_{H}=180 \mathrm{GeV}\right)$ showing cumulative acceptance times efficiency values for three subchannels $(=0$, $=1$ or $\geq 2$ central electrons). Only statistical errors are shown. . . . . .

5.22 RunIIb: Cut flow for $H \rightarrow Z Z \rightarrow e e \mu \mu \mathrm{MC}$ signal $\left(m_{H}=125 \mathrm{GeV}\right)$ showing cumulative acceptance times efficiency values for three subchannels ( $=0,=1$ or $\geq 2$ central electrons). Only statistical errors are shown. 76

5.23 RunIIb: Cut flow for $Z H \rightarrow e e \mu \mu \mathrm{MC}$ signal $\left(m_{H}=125 \mathrm{GeV}\right)$ showing cumulative acceptance times efficiency values for three subchannels $(=0$, $=1$ or $\geq 2$ central electrons). Only statistical errors are shown. . . . . .

5.24 RunIIb: Cut flow for $H \rightarrow Z Z \rightarrow e e \mu \mu \mathrm{MC}$ signal $\left(m_{H}=180 \mathrm{GeV}\right)$ showing cumulative acceptance times efficiency values for three subchannels $(=0,=1$ or $\geq 2$ central electrons). Only statistical errors are shown. 
5.25 RunIIb: Cut flow for $Z H \rightarrow e e \mu \mu \mathrm{MC}$ signal $\left(m_{H}=180 \mathrm{GeV}\right)$ showing cumulative acceptance times efficiency values for three subchannels $(=0$, $=1$ or $\geq 2$ central electrons). Only statistical errors are shown. . . . . . .

5.26 RunIIa: Cut flow for non-resonant $Z Z \rightarrow$ eeee MC background showing cumulative acceptance times efficiency for three subchannels $(=2,=3$ or $\geq 4$ central electrons, $=1$ ICR electron) $\ldots \ldots \ldots \ldots$

5.27 RunIIb: Cut flow for non-resonant $Z Z \rightarrow$ eeee MC background showing cumulative acceptance times efficiency for three subchannels $(=2,=3$ or $\geq 4$ central electrons, $=1$ ICR electron $) \ldots \ldots \ldots \ldots$

5.28 RunIIa: Acceptance $\times$ efficiency values. . . . . . . . . . . . . . . 83

5.29 RunIIb: Acceptance $\times$ efficiency values.. . . . . . . . . . . . . . . . 83

5.30 RunII Cut flow for $\mu \mu \mu \mu$ weighted non-resonant $Z Z$ MC. . . . . . . . . 84

5.31 Cut flow for RunII $\mu \mu \mu \mu$ non-resonant $Z$ MC showing cumulative and exclusive acceptance times efficiency. . . . . . . . . . . . . . 84

5.32 Acceptance $\times$ efficiency values for the $\mu \mu \mu \mu$ Channel. . . . . . . . . . . 85

5.33 RunIIa: Cut flow for non-resonant $Z Z \rightarrow e e \mu \mu \mathrm{MC}$ background showing cumulative acceptance times efficiency for three subchannels $(=0,=1$ or $\geq 2$ central electrons) $\ldots \ldots \ldots \ldots \ldots \ldots \ldots \ldots \ldots \ldots \ldots \ldots$

5.34 RunIIb: Cut flow for non-resonant $Z Z \rightarrow e e \mu \mu \mathrm{MC}$ background showing cumulative times efficiency for three subchannels $(=0,=1$ or $\geq 2$ central

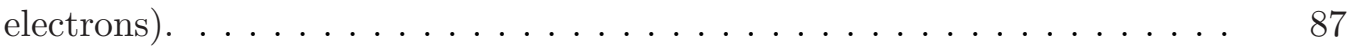

5.35 RunIIa: Acceptance $\times$ efficiency values. . . . . . . . . . . . . . . 87

5.36 RunIIa: Acceptance $\times$ efficiency values. . . . . . . . . . . . . . . 87

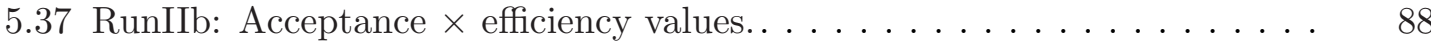

5.38 RunIIb: Acceptance $\times$ efficiency values.. . . . . . . . . . . . . . 88 
5.39 RunIIa estimated $\mathrm{Z}+$ jets background in three eeee subchannels for $3 e+$ jet, $2 e+$ jet and total event yield, where total is $3 e+$ jet minus $2 e+$ jet. . . . . .

5.40 RunIIb estimated $\mathrm{Z}+$ jets background in three eeee subchannels for $3 e+$ jet, $2 e+$ jet and total event yield, where total is $3 e+$ jet minus $2 e+$ jet. . . . . .

5.41 RunIIa $Z+$ jets background for $e e \mu \mu$ channel for the three cases of $=0,=1$ or $\geq 2 \mathrm{CC}$ objects (electron + jet) showing the $2 \mu+e+$ jet, $2 \mu+2$ jet, $2 e+2$ jet and total event yield, where the total is $2 \mu+e+$ jet minus $2 \mu+2$ jet plus

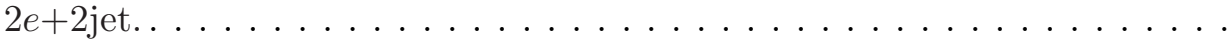

5.42 RunIIb $Z+$ jets background for $e e \mu \mu$ channel for the three cases of $=0,=1$ or $\geq 2 \mathrm{CC}$ objects (electron + jet) showing the $2 \mu+e+$ jet, $2 \mu+2$ jet, $2 e+2$ jet and total event yield, where the total is $2 \mu+e+$ jet minus $2 \mu+2$ jet plus

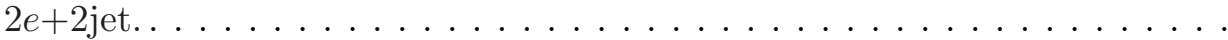

5.43 RunIIa: Rejection factors against beam halo and cosmics from dca, timing

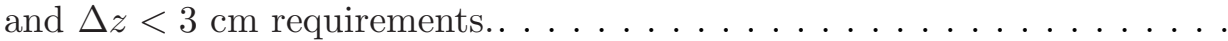

5.44 RunIIb: Rejection factors against beam halo and cosmics from dca, timing

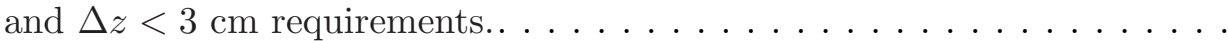

6.1 Contributions from non-negligible backgrounds in the eeee subchannels, expected (Exp.) non-resonant $Z Z$ and Higgs boson signals, and number of observed events. Uncertainties are statistical followed by systematic.. . . .

6.2 Contributions from non-negligible backgrounds in the $\mu \mu \mu \mu$ channel, expected non-resonant $Z Z$ and Higgs boson signal, and number of observed events. Uncertainties are statistical followed by systematic. . . . . . . . . . 
6.3 Contributions from non-negligible backgrounds in the $e e \mu \mu$ subchannels, plus expected signal and number of observed events. Uncertainties are statistical followed by systematic. . . . . . . . . . . . . . . . .

7.1 Relative uncertainties in the RunIIa eeee channel with 2 CC electrons for various yields and backgrounds. The top nine rows are systematic uncertainties, while the next two rows are statistical in nature, and then total uncertainty is given. . . . . . . . . . . . . . .

7.2 Relative uncertainties in the RunIIa eeee channel with 3 CC electrons for various yields and backgrounds. The top nine rows are systematic uncertainties, while the next two rows are statistical in nature, and then total uncertainty is given. . . . . . . . . . . . . . . . .

7.3 Relative uncertainties in the RunIIa eeee channel with 4 CC electrons for various yields and backgrounds. The top nine rows are systematic uncertainties, while the next two rows are statistical in nature, and then total uncertainty is given. . . . . . . . . . . . . . . . . . .

7.4 Relative uncertainties in the RunIIa eeee channel with 1 ICR electron for various yields and backgrounds. The top nine rows are systematic uncertainties, while the next two rows are statistical in nature, and then total uncertainty is given. . . . . . . . . . . . . . . . .

7.5 Relative uncertainties in the RunIIa $\mu \mu \mu \mu$ channel for various yields and backgrounds. The top eleven rows are systematic uncertainties, while the next two rows are statistical in nature, and then total uncertainty is given. 


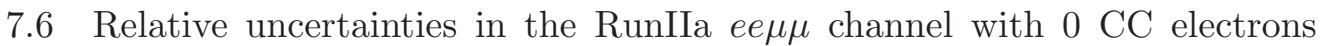
for various yields and backgrounds. The top fourteen rows are systematic uncertainties, while the next two rows are statistical in nature, and then total uncertainty is given. . . . . . . . . . . . . . . .

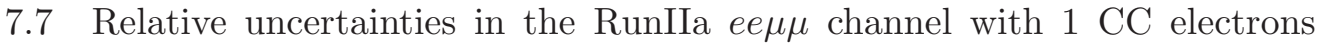
for various yields and backgrounds. The top fourteen rows are systematic uncertainties, while the next two rows are statistical in nature, and then total uncertainty is given. . . . . . . . . . . . . . . .

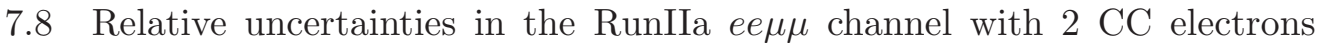
for various yields and backgrounds. The top fourteen rows are systematic uncertainties, while the next two rows are statistical in nature, and then total uncertainty is given. . . . . . . . . . . . . . .

7.9 Relative uncertainties in the RunIIb eeee channel with 2 CC electrons for various yields and backgrounds. The top nine rows are systematic uncertainties, while the next two rows are statistical in nature, and then total uncertainty is given. . . . . . . . . . . . . . . .

7.10 Relative uncertainties in the RunIIb eeee channel with $3 \mathrm{CC}$ electrons for various yields and backgrounds. The top nine rows are systematic uncertainties, while the next two rows are statistical in nature, and then total uncertainty is given. . . . . . . . . . . . . . . . .

7.11 Relative uncertainties in the RunIIb eeee channel with 4 CC electrons for various yields and backgrounds. The top nine rows are systematic uncertainties, while the next two rows are statistical in nature, and then total uncertainty is given. . . . . . . . . . . . . . . . 
7.12 Relative uncertainties in the RunIIb eeee channel with 1 ICR electron for various yields and backgrounds. The top nine rows are systematic uncertainties, while the next two rows are statistical in nature, and then total uncertainty is given. . . . . . . . . . . . . . . .

7.13 Relative uncertainties in the RunIIb $\mu \mu \mu \mu$ channel for various yields and backgrounds. The top eleven rows are systematic uncertainties, while the next two rows are statistical in nature, and then total uncertainty is given.

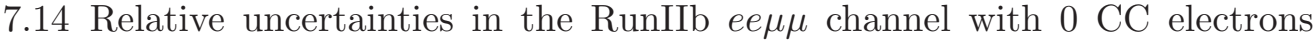
for various yields and backgrounds. The top fourteen rows are systematic uncertainties, while the next two rows are statistical in nature, and then total uncertainty is given. . . . . . . . . . . . . .

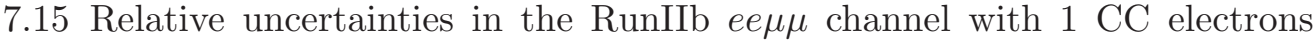
for various yields and backgrounds. The top fourteen rows are systematic uncertainties, while the next two rows are statistical in nature, and then total uncertainty is given. . . . . . . . . . . . . .

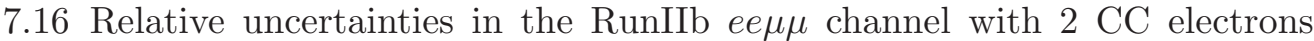
for various yields and backgrounds. The top fourteen rows are systematic uncertainties, while the next two rows are statistical in nature, and then total uncertainty is given. . . . . . . . . . . . . . . . .

8.1 Signal yields for Higgs boson masses of 125 and $180 \mathrm{GeV}$. The other $H$ decays not listed individually consist of the decays $H \rightarrow \gamma \gamma, H \rightarrow \mu \mu$, and

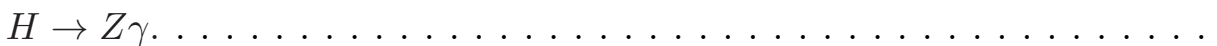

8.2 Expected and observed 95\% C.L. upper limits on the SM Higgs boson production cross section relative to the value expected in the SM.. . . . . . 


\section{LIST OF FIGURES}

$\begin{array}{lll}\text { Figure } & \text { Page }\end{array}$

1.1 The minima of the potential for the case of $\mu^{2}>0$ (left) and $\mu^{2}<0$ (right) for a scalar field. . . . . . . . . . . . . . . . . . . 11

1.2 Higgs decay branching fractions as a function of Higgs masses [7].. . . . . 13

1.3 Higgs decay to an "off-shell" $W$ or $Z \ldots \ldots \ldots \ldots \ldots \ldots$

1.4 Indirect search for the Higgs boson: $\Delta \chi^{2}$ (black line) of the fit to the EW parameters (top quark and $W$ boson masses) as a function of the Higgs masses. The yellow band represents excluded Higgs masses at 95\% CL. . .

1.5 2012 Tevatron limits on SM Higgs production. Mass regions of $147-180$ $\mathrm{GeV}$ and 100 - $103 \mathrm{GeV}$ has been excluded at 95\% confidence level and a $2.5 \sigma$ evidence of production has been reported. . . . . . . . . 15

1.6 2012 CMS Higgs production result in the $H \rightarrow Z Z \rightarrow 4$ lepton channel. . 16

1.72012 ATLAS Higgs production result in the $H \rightarrow \gamma \gamma$ channel. . . . . . . . 16

2.1 Coordinate System. . . . . . . . . . . . . . . . . . . . . . 19

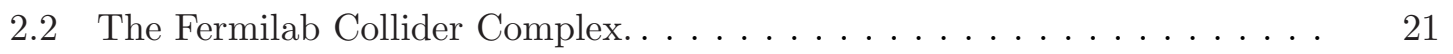

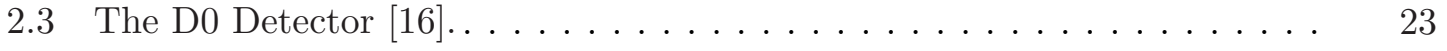

2.4 The D0 Detector highlighting upgrades for RunII [16] . . . . . . . . . 24

$2.5 r-z$ view of the tracking system $[16] \ldots \ldots \ldots \ldots \ldots \ldots \ldots$ 
2.6 The D $\varnothing$ Silicon Microstrip Tracker $[17] \ldots \ldots \ldots \ldots \ldots$

2.7 The central and forward preshower detectors. $[16] \ldots \ldots \ldots \ldots \ldots$

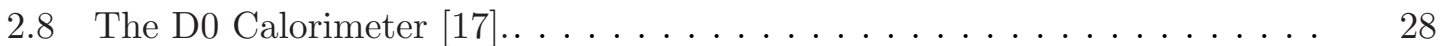

2.9 Schematic view of a portion of the D0 calorimeters showing the transverse and longitudinal segmentation pattern [17]. . . . . . . . . . . . . 29

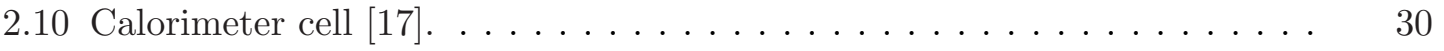

2.11 The DØ Muon System $(\mathrm{PDT}+\mathrm{MDT})[20] \ldots \ldots \ldots \ldots \ldots \ldots$

2.12 The DØ Muon System (Scintillators) [17] . . . . . . . . . . . . . . 34

2.13 Overview of the $\mathrm{D} \varnothing$ trigger and data acquisition systems [17] . . . . 36

2.14 Block diagram of the D $\varnothing$ L1 and L2 trigger systems. The arrows show the flow of trigger-related data $[17] \ldots \ldots \ldots \ldots \ldots$

5.1 The $p_{T}$ distributions of the (a) lead, (b) second, (c) third, and (d) trailing electrons in four electron events, with data, expected Higgs signal (125 and $180 \mathrm{GeV})$ and backgrounds superimposed. . . . . . . . . . . . .

5.2 The $p_{T}$ of the lead and trailing ee pair in four electron events, where of the three potential sets of di-electron invariant masses in each event, we show only the one that has one of the pair masses closest to the $Z$ mass. . . . .

5.3 The di-electron (right) and four electron (left) mass distribution. As there are three potential sets of di-electron invariant masses in each event, we show only the one that has one of the pair masses closest to the $Z$ mass. .

5.4 The $p_{T}$ distributions of the four muons ordered in $p_{T}$ in the $4 \mu$ channel, with data, expected Higgs signal (125 and $180 \mathrm{GeV}$ ) and backgrounds superimposed. . . . . . . . . . . . . . . . . . 
5.5 The $p_{T}$ distributions of leading $Z / \gamma^{*}$ and second $Z / \gamma^{*}$ in the $4 \mu$ channel, with data, expected Higgs signal (125 and $180 \mathrm{GeV}$ ) and backgrounds superimposed. . . . . . . . . . . . . . . . . . . . . .

5.6 The distributions of the di-muon invariant mass of the best pair and four muon invariant mass in the $\mu \mu \mu \mu$ channel, with data, expected Higgs signal (125 and $180 \mathrm{GeV})$ and backgrounds superimposed. . . . . . . . . .

5.7 Distributions of the lepton $p_{T}$ ordered in $p_{T}$ for data, expected Higgs signal (125 and $180 \mathrm{GeV}$ ) and backgrounds superimposed for the ee $\mu \mu$ channel..

5.8 Distributions of dilepton invariant mass and four lepton invariant mass for data, expected Higgs signal (125 and $180 \mathrm{GeV}$ ) and backgrounds superimposed for the $e e \mu \mu$ channel. . . . . . . . . . . . . . . .

$6.1-\ln (L)$ versus cross section $($ in $\mathrm{pb}) . \ldots \ldots \ldots \ldots \ldots \ldots \ldots \ldots \ldots$

6.2 Distributions of lepton $p_{T}$ ordered in $p_{T}$ in data, expected Higgs signal and backgrounds superimposed for the combined channels. . . . . . . . . . . .

6.3 Distributions of $\Delta R$ and $\Delta \phi$ between the two leptons in each $Z$ decay in data, expected Higgs signal and backgrounds superimposed for the combined channels. . . . . . . . . . . . . . . . . . . . .

6.4 Distributions of missing transverse energy, the $p_{T}$ of the $Z Z$ system, dilepton invariant mass and four lepton invariant mass in data, expected Higgs signal and backgrounds superimposed for the combined channels. . . . . . 
8.1 Distributions of the COLLIE inputs in the eeee (top left), $\mu \mu \mu \mu$ (top right), $е e \mu \mu$ (bottom left) and all channels combined (bottom right) for data, non-resonant $Z Z, Z+$ jets, migration and the Higgs boson with mass of $125 \mathrm{GeV}$. The bins between 0 and 500 are the four-lepton invariant mass between 0 and $500 \mathrm{GeV}$ with $\mathscr{E}_{T}<30 \mathrm{GeV}$. The bins between 500 and 650 are the $\mathscr{E}_{T}$ of events with $\mathbb{E}_{T}>30 \mathrm{GeV}$; each bin corresponds to the

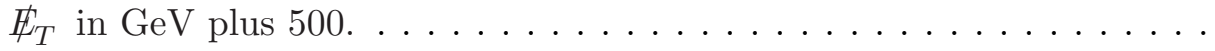

8.2 Distributions of the COLLIE inputs for each individual four electron subset for data, non-resonant $Z Z, Z+$ jets, migration and the Higgs boson with mass of $125 \mathrm{GeV}$. The $4 \mathrm{CC}$ (top left), $3 \mathrm{CC}$ (top right), and $2 \mathrm{CC}$ (bottom left) subsets have no ICR electrons. The 1 ICR(bottom right) subset has exactly 1 ICR electron and at least $2 \mathrm{CC}$ electrons. The bins between 0 and 500 are the four-lepton invariant mass between 0 and $500 \mathrm{GeV}$ with $E_{T}<30 \mathrm{GeV}$. The bins between 500 and 650 are the $\mathscr{E}_{T}$ of events with $\mathbb{E}_{T}>30 \mathrm{GeV}$; each bin corresponds to the $\mathbb{E}_{T}$ in $\mathrm{GeV}$ plus $500 . \ldots \ldots$

8.3 Distributions of the COLLIE inputs for each individual ee $\mu \mu$ subset for data, non-resonant $Z Z, Z+$ jets, migration and the Higgs boson with mass of $125 \mathrm{GeV}$. The 2CC (top left) subset has two CC electrons and two muons. The 1 CC (top right) subset has one CC electron, one EC electron, and two muons. The $0 \mathrm{CC}$ subset (bottom) has two EC electrons and two muons. The bins between 0 and 500 are the four-lepton invariant mass between 0 and $500 \mathrm{GeV}$ with $\mathbb{E}_{T}<30 \mathrm{GeV}$. The bins between 500 and 650 are the $\mathbb{E}_{T}$ of events with $\mathbb{E}_{T}>30 \mathrm{GeV}$; each bin corresponds to the $\mathbb{E}_{T}$ in $\mathrm{GeV}$

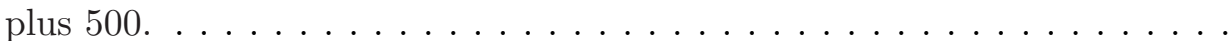


8.4 The (a) expected and observed 95\% C.L. upper limits on the SM Higgs boson production cross section relative to the value expected in the SM, and the (b) log-likelihood ratio for all four lepton channels combined. . . .

A.1 RunIIa: Plot of the measured electron fakerates. The left plot shows the fakerate as a function of jet $p_{T}$. The right plot shows shows the fakerate as a function of jet $\eta_{\text {det }} \ldots \ldots \ldots \ldots \ldots \ldots \ldots \ldots \ldots \ldots \ldots$

A.2 RunIIb: Plot of the measured electron fakerates. The left plot shows the fakerate as a function of jet $p_{T}$. The right plot shows shows the fakerate as a function of jet $\eta_{\text {det }} \ldots \ldots \ldots \ldots \ldots \ldots \ldots \ldots \ldots \ldots \ldots \ldots \ldots$

A.3 Plot of the measured ICR electron fakerate vs. $\mathbb{E}_{T} \ldots \ldots \ldots \ldots$

A.4 RunIIa: Plot of the measured ICR electron fakerates. The left plot shows the fakerate as a function of jet $p_{T}$. The right plot shows shows the fakerate

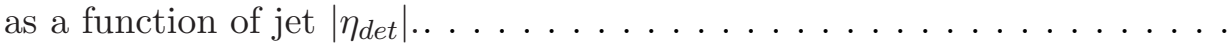

A.5 RunIIb: Plot of the measured ICR electron fakerates. The left plot shows the fakerate as a function of jet $p_{T}$. The right plot shows shows the fakerate

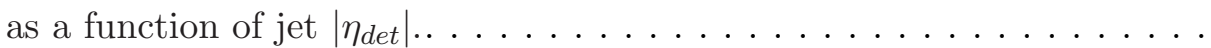

B.1 RunIIa plots of the measured muon fakerates. The plots show the fakerate as a function of jet $p_{T}$ for muons with $p_{T}>15 \mathrm{GeV}$ when all muons are isolated for different values of nseg. . . . . . . . . . . . .

B.2 RunIIa plots of the measured muon fakerates. The plots show the fakerate as a function of jet $\eta_{\text {det }}$ for muons with $p_{T}>15 \mathrm{GeV}$ when all muons are isolated for different values of nseg. . . . . . . . . . . . . 
B.3 RunIIb plots of the measured muon fakerates. The plots show the fakerate as a function of jet $p_{T}$ for muons with $p_{T}>15 \mathrm{GeV}$ when all muons are isolated for different values of nseg. . . . . . . . . . . . . . . .

B.4 RunIIb plots of the measured muon fakerates. The plots show the fakerate as a function of jet $\eta_{\text {det }}$ for muons with $p_{T}>15 \mathrm{GeV}$ when all muons are isolated for different values of nseg. . . . . . . . . . . . . . . 


\section{LIST OF APPENDICES}

Appendix $\quad$ Page

A ELECTRON FAKERATES . . . . . . . . . . . . . . . . . . . . . . 140

B MUON FAKERATES . . . . . . . . . . . . . . . . . . . . . . . . 144 


\section{CHAPTER 1 INTRODUCTION}

\subsection{The Standard Model of Particle Physics}

The Standard Model (SM) $[1,2]$ is the quantum field theory of subatomic particles and their interactions. In the SM particles are divided into fermions and gauge bosons. Fermions have spin-1/2, follow Fermi-Dirac statistics, and correspond to the matter content of the SM. Fermions themselves are subdivided into quarks and leptons, with each group ordered in three families or generations. In contrast to leptons, quarks are not found free in nature. They form bound states of either three quarks (baryons) or quark-antiquark pairs (mesons). Baryons and mesons are generically called hadrons. Additionally, to each fermion in the SM there is a corresponding antifermion. All fermions are shown in Table 1.1.

Gauge bosons are spin-1 particles, follow Bose-Einstein statistics, and are the mediators

of the interactions among fermions. SM gauge bosons are summarized in Table 1.2 below.

The interactions in the SM are the electromagnetic, weak nuclear and strong nuclear forces. The SM is based on the gauge symmetry $S U(3)_{C} \otimes S U(2)_{L} \otimes U(1)_{Y}$, where $S U(N)_{C}$ means special unitary group of degree $N, C$ and $Y$ stands respectively for color charge and weak hypercharge quantum numbers, and $L$ means that the symmetry only applies to left-handed fermions. The gauge bosons in the SM are the photon $(\gamma)$, which carries the 
electromagnetic force, eight gluons $(g)$, that mediate the strong nuclear force, and three weak bosons $\left(W^{ \pm}\right.$and $Z$ ) as the mediators of the weak nuclear force. $S U(3)_{C}$ represents the strong force, called Quantum Chromodynamics (QCD) while $S U(2)_{L} \otimes U(1)_{Y}$ is used for electroweak interactions, including the Quantum Electrodynamics (QED).

Table 1.1: The known quarks and leptons.

\begin{tabular}{|cc|cc|cc|cc|}
\hline \multicolumn{2}{|c|}{ Quarks } & \multicolumn{3}{c|}{ Leptons } \\
\hline \multicolumn{2}{|c|}{ Charge 2/3 } & \multicolumn{2}{c|}{ Charge $-1 / 3$} & \multicolumn{2}{c|}{ Charge -1} & \multicolumn{2}{c|}{ Charge 0 } \\
\hline & Mass, GeV & \multicolumn{2}{|c|}{ Mass, GeV } & \multicolumn{2}{|c|}{ Mass, GeV } & \multicolumn{2}{c|}{ Mass } \\
\hline$u$ & $0.001-0.005$ & $d$ & $0.003-0.009$ & $e$ & 0.000511 & $\nu_{e}$ & $<3 \mathrm{eV}$ \\
$c$ & $1.15-1.35$ & $s$ & $0.075-0.175$ & $\mu$ & 0.106 & $\nu_{\mu}$ & $<190 \mathrm{keV}$ \\
$t$ & $172.5 \pm 2.7$ & $b$ & $4.0-4.4$ & $\tau$ & 1.777 & $\nu_{\tau}$ & $<18.2 \mathrm{MeV}$ \\
\hline
\end{tabular}

Table 1.2: Gauge bosons

\begin{tabular}{|c|c|c|}
\hline Boson & Charge & Mass $(\mathrm{GeV})$ \\
\hline photon $(\gamma)$ & 0 & massless \\
\hline gluon $(g)$ & 0 & massless \\
\hline$W^{ \pm}$ & \pm 1 & $80.40 \pm 0.02$ \\
\hline$Z$ & 0 & $91.19 \pm 0.01$ \\
\hline
\end{tabular}

Although gravity is one of the fundamental forces in nature, its effects can be safely neglected in all particle experiments at present energies. Since there is no consistent quantum theory of gravity as to-date it is not described in the SM framework. SM forces are summarized in Table 1.3.

The way particles and interaction are organized in the SM reflects the way particles interact. Besides electric charge and weak isospin, quarks carry an extra quantum number named color, which represents the strong interaction charge. Thus quarks experience electromagnetic, weak nuclear and strong nuclear forces. The remaining six fermions - lep- 
Table 1.3: Fundamental interactions

\begin{tabular}{|c||c|c|c|}
\hline Interaction & Gauge boson & Range & Acts on \\
\hline Gravitational & graviton(?) & $\infty$ & all particles \\
\hline Eletromagnetic & photon $\gamma$ & $\infty$ & charged particles \\
\hline Weak & $W^{ \pm}$and $Z$ & $<10^{-18} \mathrm{~m}$ & leptons and quarks \\
\hline Strong & gluons $g$ & $\approx 10^{-15} \mathrm{~m}$ & hadrons \\
\hline
\end{tabular}

tons - are also found in six different kinds. Three of them have negative charge and weak isospin, and they experience both electromagnetic and weak nuclear forces. The remaining three are neutral and are named neutrinos and only experience the weak nuclear force. For many years neutrinos were thought to be massless until experiments showed they indeed have small masses. However at high energies the assumption that they are massless is a very good approximation.

\subsubsection{The Electroweak Sector}

The Lagrangian of a Yang-Mills theory can be written as

$$
\mathcal{L}_{\text {Yang-Mills }}=\mathcal{L}_{\text {fermions }}+\mathcal{L}_{\text {gauge }}
$$

In this sense we write the electroweak lagrangian as [3]:

$$
\mathcal{L}_{\text {Electroweak }}=\sum_{k} i \bar{\psi}_{k} \gamma_{\mu} D^{\mu} \psi_{k}-\frac{1}{4} W_{\mu \nu}^{a} W^{a \mu \nu}-\frac{1}{4} B_{\mu \nu} B^{\mu \nu}
$$

where $D_{\mu}=\partial_{\mu}-i g \vec{\sigma} / 2 \vec{A}-i g^{\prime} \frac{Y}{2} B_{\mu}, F_{\mu \nu}^{a}=\partial_{\mu} A_{\nu}^{a}-\partial_{\nu} A_{\mu}^{a}+g \epsilon^{a b c} A_{\mu}^{b} A_{\nu}^{c}, a=1,2,3$, and $B_{\mu \nu}=\partial_{\mu} B_{\nu}-\partial_{\nu} B_{\mu}$. A and B are the gauge fields, $\psi_{k}$ represents the interacting fermions 
and $\mathrm{Y}$ is the multiplet hypercharge given by $Y=2\left(Q-T_{3}\right)$, where $T_{3}$ is the third component of weak isospin.

Both QED and QCD are invariant under $U(1)$ and $S U(3)$ gauge transformations respectively. These invariances lead to the photon and gluon gauge bosons. So, we could expect that electroweak to be invariant under the $S U(2)_{L}$ transformation (the subscript $L$ means that the weak isospin current couples to left-handed fermions only). However, when it is required we run into some serious problems.

First, as stated above, the two charged bosons only couple to left-handed fermions (and right-handed antifermions). The neutral boson itself couples differently to right-handed and left-handed fermions. In principle the neutral boson should not have a right-handed component. Second, the $S U(2)_{L}$ invariance requires that all three bosons are massless, which is in a complete disagreement with experiments that measure their masses.

The answer for these problems is in the fact that at energies high enough $(\sim 100 \mathrm{GeV})$ both weak and electromagnetic interactions are unified into a single electroweak interaction that is based on the $S U(2)_{L} \otimes U(1)_{Y}$ symmetry group. Requiring the electroweak Lagrangean to be invariant under a $S U(2)_{L} \otimes U(1)_{Y}$ transformation makes it consistent with experimental observations. Electroweak symmetry is spontaneously broken as

$$
S U(2)_{L} \otimes U(1)_{Y} \rightarrow U(1)_{E M}
$$

and the introduction of the Higgs field gives mass to the weak gauge bosons.

The first electroweak group is the weak isospin $S U(2)_{L}$ group. All left-handed fermions transform as doublets under $S U(2)_{L}$ : 


$$
\left(\begin{array}{c}
u_{L} \\
d_{L}
\end{array}\right),\left(\begin{array}{c}
c_{L} \\
s_{L}
\end{array}\right),\left(\begin{array}{c}
t_{L} \\
b_{L}
\end{array}\right),\left(\begin{array}{c}
\nu_{e} \\
e_{L}
\end{array}\right),\left(\begin{array}{c}
\nu_{\mu} \\
\mu_{L}
\end{array}\right),\left(\begin{array}{c}
\nu_{\tau} \\
\tau_{L}
\end{array}\right)
$$

The generators of the $S U(2)$ group obey the Lie algebra

$$
\left[T_{a}, T_{b}\right]=i \epsilon_{a b c} T_{c}
$$

for this representation and are the Pauli matrices:

$$
T^{a}=\sigma^{a} / 2, \quad(a=1,2,3)
$$

with corresponding vector gauge bosons

$$
W_{\mu}^{a}, \quad(a=1,2,3)
$$

and a coupling constant $g$. The right-handed fermions

$$
e_{R}, \mu_{R}, \tau_{R}, u_{R}, c_{R}, t_{R}, d_{R}, s_{R}, b_{R}
$$

are singlets and are invariant under $S U(2)_{L}$ transformations. The second group is $U(1)_{Y}$ and is known as the weak hypercharge group and has a coupling constant $g^{\prime}$ and a vector boson $B_{\mu}$.

The first two bosons of $S U(2)_{L}$ are charged and then are identified with the $W^{ \pm}$bosons of the weak interactions:

$$
W_{\mu}^{ \pm}=\frac{1}{\sqrt{2}}\left(W_{\mu}^{1} \mp i W_{\mu}^{2}\right)
$$


As the other vector bosons, $W_{\mu}^{3}$ and $B_{\mu}$ are neutral, but cannot be identified as the $Z$ boson and the photon respectively. Due to spountaneous symmetry breaking they mix and both the $Z$ and the photon are linear combinations of them:

$$
\begin{aligned}
& Z=\cos \theta_{W} W_{\mu}^{3}-\sin \theta_{W} B \\
& A=\sin \theta_{W} W_{\mu}^{3}+\cos \theta_{W} B
\end{aligned}
$$

where $\theta_{W}$ is the weak mixing angle or Weinberg angle, which is determined by the coupling constants with

$$
\tan \theta_{W}=\frac{g^{\prime}}{g}
$$

\subsubsection{The QCD Sector}

Quantum Chromodynamics (QCD) is the theory of the nuclear strong interaction in the SM. It is a non-Abelian gauge theory based on a Yang-Mills theory with gauge group $S U(3)_{c}$ and describes the interactions of quarks and gluons.

The group $S U(3)_{c}$ has eight generator matrices $T^{a}$, with each one corresponding to a gauge vector boson, named gluon, represented by a field $G_{\mu}^{a}$, where $a=1, . ., 8$. The color charge quantum number $c$ occurs in three different flavors: red, blue and green. As gluons carry color charge they interact among themselves and when exchanged between quarks they change quarks flavors as well. All of this is described in the QCD Lagrangian [3]: 


$$
\mathcal{L}_{Q C D}=\sum_{j} i \bar{q}_{j} \gamma^{\mu} D_{\mu} q_{j}-\frac{1}{2} \operatorname{tr} G_{\mu \nu} G^{\mu \nu}
$$

where $q_{j}$ are the quark fields, with $j$ running over all flavors, $D_{\mu}=\partial_{\mu}-i g_{s} G_{\mu}, G_{\mu \nu}=$ $\partial_{\mu} G_{\nu}-\partial_{\nu} G_{\mu}-i g\left[G_{\mu}, G_{\nu}\right]$ and $G_{\mu}=\sum_{a=1}^{8} G_{\mu}^{a} \lambda^{a} / 2$, where $\lambda^{a}$ are the Gell-Mann matrices that satisfy the relations $\left[\lambda^{a} / 2, \lambda^{b} / 2\right]=i f^{a b c} \lambda^{c} / 2$ and $\operatorname{tr}\left(\lambda^{a} \lambda^{b}\right)=2 \delta^{a b}$, and $G_{\mu}^{a}$ are the gluon field operators.

Perhaps the most stringent feature of QCD is that, in contrast with electromagnetism, the force between two particles is directly proportional to the distance between them. This behaviour leads to the so called asymptotic freedom, where at short distances (higher energies) quarks behave almost as free particles and to the so called confinement in QCD, which means that there no free quarks in nature, only bound states of quarks. These bound states are called baryons if there are three quarks or mesons if a quark-antiquark pair is formed.

As in quantum electrodynamics (QED) we also define a coupling for QCD. This coupling constant is known as $g_{3}$ and it is associated with the $S U(3)_{c}$ gauge interactions. This coupling depends on the normalizations scale $\mu$ and is more commonly written as:

$$
\alpha_{s}=\frac{g_{3}^{2}}{4 \pi}
$$

and $\alpha_{s}$ is the analog of the fine structure constant in QED. As in QED we have to take into account (non-Abelian) corrections to the QCD potential in order to calculate $\alpha_{s}$. This is done using pertubative calculations. In lowest order it is given by [2]:

$$
\alpha_{s}\left(\mu^{2}\right)=\frac{\alpha_{s}\left(\mu_{0}\right)}{1-\frac{b_{0} \alpha_{s}\left(\mu_{0}\right)}{2 \pi} \ln \left(\mu / \mu_{0}\right)}
$$


with

$$
b_{0}=-11+\frac{2}{3} n_{f}
$$

where $n_{f}$ is the number of flavors with mass below $\mu$. Since $b_{0}$ assume negative values, $\alpha_{s}$ blows up if $\mu$ is small enough. Another way to see this behaviour is defining

$$
\Lambda_{Q C D}=\mu_{0} e^{2 \pi / b_{0} \alpha_{s}\left(\mu_{0}\right)}
$$

which allows us to write (1.15) as

$$
\alpha_{s}\left(\mu^{2}\right)=\frac{2 \pi}{b_{0} \ln \left(\mu / \Lambda_{Q C D}\right)}
$$

Here we explicitly see that when $\mu=\Lambda_{Q C D}$ the running coupling constant diverges, namely, at high energies $\alpha_{s}$ becomes small and pertubative QCD methods can be safely employed to describe the asymptotic freedom regime in QCD. On the other hand, as energies decreases and the value of $\alpha_{s}$ approaches that of $\Lambda_{Q C D}$, perturbative methods no longer apply, leaving the description of quark confinement incomplete.

Despite all complexity posed by the non-perturbative regime, it is still possible to calculate matrix elements and cross sections in QCD. Generically, all particles inside a hadron, i.e., quarks, antiquarks and gluons are called partons and the model that describe them is named the parton model. When the momentum exchanged between a probe, a high energy photon for instance, and a parton is much higher than $\Lambda_{Q C D}$, the total cross section can be written as the incoherent sum of the individual cross sections on each parton. If we assume that each parton carries a fraction $x$ of the momentum of the original hadron we define the so called parton distribution functions or PDF's $f_{i}^{p}(x)$ which is the probability of finding a parton $p$ carrying a fraction $x$ of the momentum of the hadron off which the 
probe is scatered. Perturbative QCD then allows us to separate hard processes in probeparton interactions from soft hadronization processes that occur later on. The parton level cross section is integrated over all possible momentum fractions distributions in order to calculate the total probe-hadron cross section. This method is called factorization.

More generally, if we assume that two hadrons $A$ and $B$ collide at a center of mass energy of $\sqrt{s}$, with their partons $a$ and $b$ at an energy $\sqrt{\hat{s}}$, and that $\sqrt{\hat{s}} \gg \Lambda_{Q C D}$ the total inelastic crosse section is written as [4]:

$$
\sigma_{A B}(s, \mu)=\sum_{a, b} \int \mathrm{d} \hat{s} \mathrm{~d} x_{1} \mathrm{~d} x_{2} f_{a}^{p}\left(x_{1}, \mu\right) f_{b}{ }^{p}\left(x_{2}, \mu\right) \hat{\sigma}_{a b}(\hat{s}, \mu) \delta\left(x_{1} x_{2} s-\hat{s}\right),
$$

where $a$ and $b$ run over all quark, antiquark and gluon types, with partons $a$ and $b$ carrying momentum fractions $x_{1}$ and $x_{2}$ of the original hadron.

PDF's are themselves functions of $\mu$. As it is not possible to derive them from the proton Hamiltonian given the non-perturbative regime involved in the calculation, they are measured in experiments and then used in Eq. 1.19. Collaborations such as CTEQ and MSTW perform such a task. Uncertainty associated with PDf's will be later dicussed in Chap. 7.

\subsubsection{The Standard Model Higgs Mechanism}

In the previous section we saw that the observation that the bosons $W^{ \pm}$and $Z$ are massive implies that the gauge invariance $S U(2)_{L} \otimes U(1)_{Y}$ does not hold. To account for this spontaneous symmetry breaking the Higgs boson is introduced [5]. The Higgs boson 
is a component of the Higgs field, which is a complex scalar isospin doublet with weak hypercharge $Y_{\Phi}=+1 / 2$ :

$$
\Phi=\left(\begin{array}{c}
\phi^{+} \\
\phi^{0}
\end{array}\right)
$$

where $\phi^{+}$and $\phi^{0}$ are complex scalar fields with charges +1 and 0 respectively. Under gauge transformations $\Phi$ transforms as :

$$
\begin{aligned}
& S U(2)_{L}: \quad \Phi(x) \rightarrow \Phi^{\prime}(x)=e^{i \theta^{a}(x) \sigma^{a} / 2} \Phi(x), \\
& U(1)_{Y}: \quad \Phi(x) \rightarrow \Phi^{\prime}(x)=e^{i \theta^{a}(x) / 2} \Phi(x) .
\end{aligned}
$$

In addtion, $\bar{\Phi} \Phi$ and $D^{\mu} \bar{\Phi} D_{\mu} \Phi$ are gauge singlets. This allows us to write a gauge-invariant potential as:

$$
V(\Phi, \bar{\Phi})=\mu^{2} \bar{\Phi} \Phi+\lambda(\bar{\Phi} \Phi)^{2}
$$

and the Lagrangian density as [3]:

$$
\mathcal{L}=\underbrace{D^{\mu} \bar{\Phi} D_{\mu} \Phi}_{\text {kinetic }}-\underbrace{V(\Phi, \bar{\Phi})}_{\text {potential }} .
$$

The potential for $V(\Phi, \bar{\Phi})$ is shown in Fig. 1.1 for the case of $\mu^{2}>0$ and $\mu^{2}<0$. When $\mu^{2}>0$ the minimum of the potential is at $\Phi=0$ and the Lagrangian describes a scalar field of mass $\sqrt{2} \mu$. But when $\mu^{2}<0$ there is a unstable maximum at $\Phi=0$ and the minimum lies on a circle, $V$ has a non-zero local minimum (vacuum expectation value - VEV) $v=\sqrt{-\mu^{2}} / \lambda$ and this is the point where pertubation calculations should be expanded around. From the figure we see that $\Phi= \pm v$. By choosing one of these values 

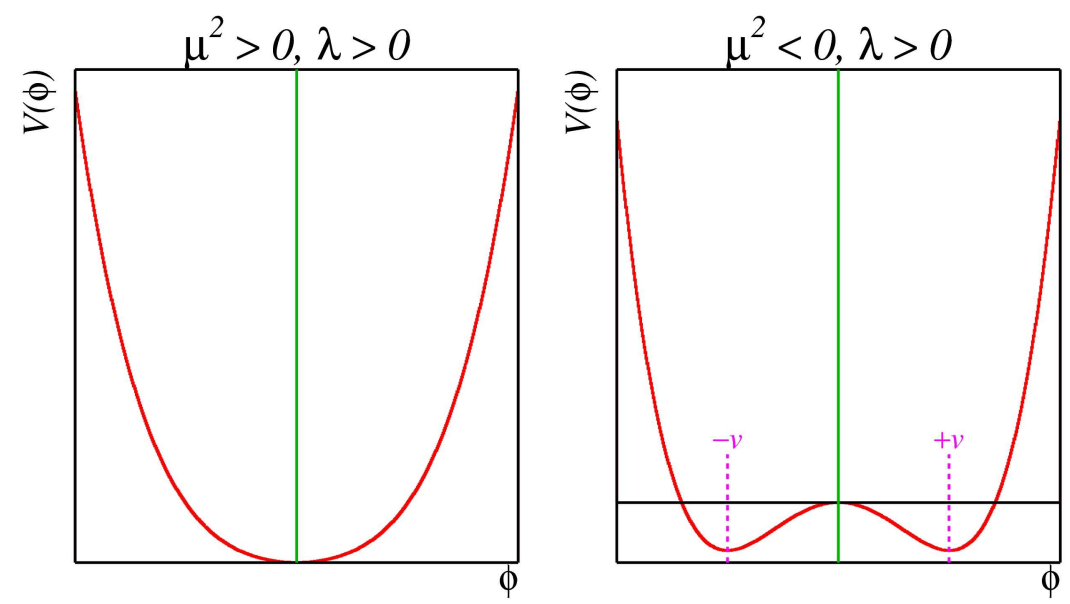

Figure 1.1: The minima of the potential for the case of $\mu^{2}>0$ (left) and $\mu^{2}<0$ (right) for a scalar field.

we are breaking the symmetry of the theory. Usually, we choose the VEV of $\Phi$ to be real and in the neutral component of the Higgs field at the same time we set $\phi^{+}=0$ :

$$
<0|\Phi| 0>=\left(\begin{array}{c}
0 \\
\frac{v}{\sqrt{2}}
\end{array}\right)
$$

Next, we can expand the non-zero component around the minimum value as:

$$
\Phi(x)=\left(\begin{array}{c}
0 \\
\frac{v+h(x)}{\sqrt{2}}
\end{array}\right) .
$$

In order to visualize the mass terms we plug Eq. 1.24 back into the kinetic term:

$$
\underbrace{D^{\mu} \bar{\Phi} D_{\mu} \Phi}_{\text {kinetic }}=\frac{1}{2} \partial_{\mu} h \partial^{\mu} h+\frac{(v+h)^{2}}{4}\left[g^{2} W^{+} \mu W^{-\mu}+\frac{1}{2}\left(g^{2}+g^{\prime 2}\right) Z_{\mu} Z^{\mu}\right] .
$$


The terms proportional to $v^{2}$ are the (mass) $)^{2}$ terms for the bosons $W^{ \pm}$and $Z$. Since there is no mass term for the photon field $A^{\mu}$ it remains massless. More explictly we have

$$
m_{W}^{2}=\frac{v^{2} g^{2}}{4} \text { and } m_{Z}^{2}=\frac{\left(v^{2}\left(g^{2}+g^{\prime 2}\right)\right)}{4}
$$

Current experimental values give for the vector bosons masses are

$$
m_{W}=80.399 \pm 0.023 \text { and } m_{Z}=91.1876 \pm 0.0021
$$

$[6]$.

In this model, fermions acquire masses by introducting a Yukawa coupling like $g_{f}^{Y} f_{L} \Phi f_{R}$, where $L$ and $R$ denotes left and right-handed fermions respectively and $g_{f}^{Y}$ is the Yukawa coupling. Thus, each fermion mass is given by:

$$
m_{f}=g_{f}^{Y} v \sqrt{2}
$$

All SM bosons would be massless if all the symmetries were intact. But while $U(1)$ and $S U(3)_{C}$ symmetries are exact, the $S U(2)_{L}$ symmetry is spontaneously broken in nature. It is postulated that the $S U(2)_{L}$ symmetry is broken by a complex scalar (spin-0) field that is a $S U(2)_{L}$ doublet, and has a non-zero expectation value in the lowest energy state of nature. After three of the four degrees of freedom of such a Higgs field are expanded in giving masses to the $W$ and $Z$ bosons and leaving the photon and gluons massless, one is left to manifest itself as a physical particle, called the Higgs boson. In the SM fermions also acquire mass by interacting with the Higgs field. 


\subsubsection{The Standard Model Higgs Boson Production}

Searches for the Higgs boson are performed through its decay to other particles. Higgs searches are divided into a low mass and a high mass region divided approximately where the Higgs mass is twice the $W$ mass. If $m_{H}<130 \mathrm{GeV}$ the preferred decay in the SM is $H \rightarrow b \bar{b}$. In this case the decays $H \rightarrow \tau \bar{\tau}$ and $H \rightarrow c \bar{c}$ also become relevant, though their detection is more difficult due to difficulties to separate them from the high QCD background. Other important decay modes are $H \rightarrow g \bar{g}$ and $H \rightarrow \gamma \bar{\gamma}$ when higher order corrections are taken into account (Fig. 1.2).

If $m_{H}<2 m_{W}$ and $2 m_{Z}$, the decays $H \rightarrow W^{-} W^{+}$and $H \rightarrow Z Z$ become important when one of the $W^{\prime}$ or one of the $Z^{\prime}$ is produced "off-shell" (Fig. 1.3).

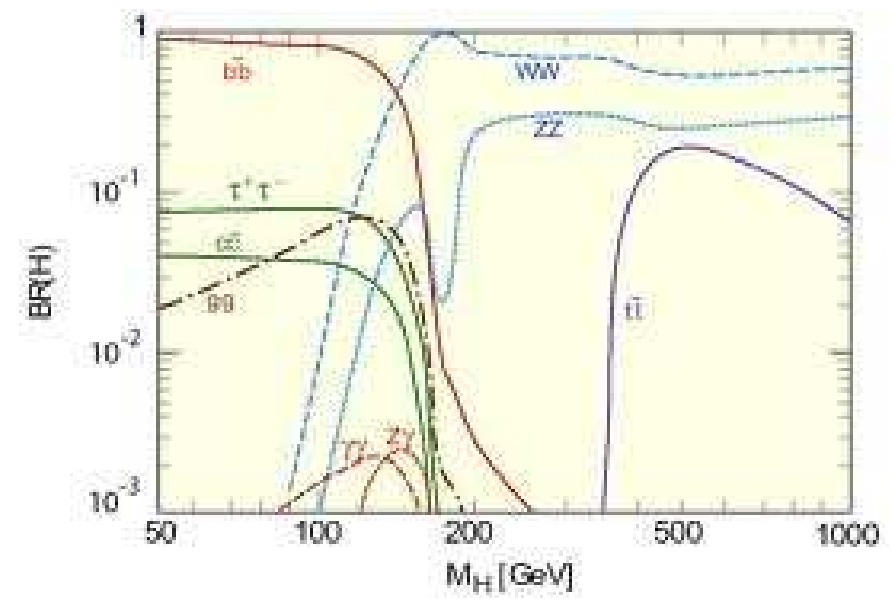

Figure 1.2: Higgs decay branching fractions as a function of Higgs masses [7].

LEP Searches: LEP experiments performed Higgs searches in electron-positron collisions with $\sqrt{s}=189-209 \mathrm{GeV}$ using $2.461 \mathrm{fb}^{-1}$ of data. The main process was $e^{+} e^{-} \rightarrow H Z$, where all $Z$ decays were included for the $H$ to $b \bar{b}$ channel, but only $Z$ decays to quarks were included for the $H$ to $\tau^{+} \tau^{-}$channel. Fig. 1.4 shows a $\Delta \chi^{2}$ (black line) for the fit to 

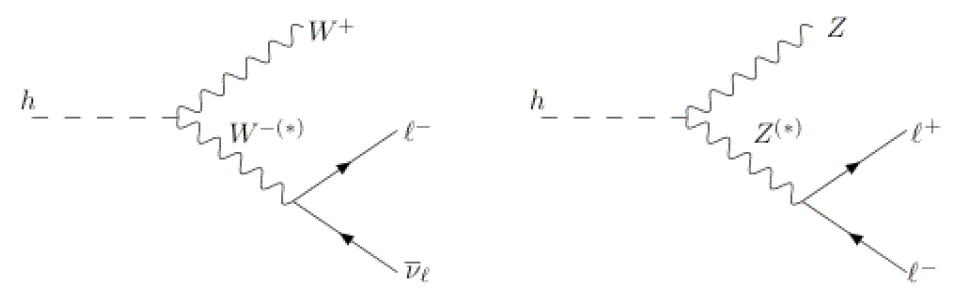

Figure 1.3: Higgs decay to an "off-shell" $W$ or $Z$.

the Higgs boson mass from electroweak measurements (top quark and $W$ boson masses). The yellow regions shows a lower limit of $114.4 \mathrm{GeV}$ at $95 \% \mathrm{CL}$ [8] .

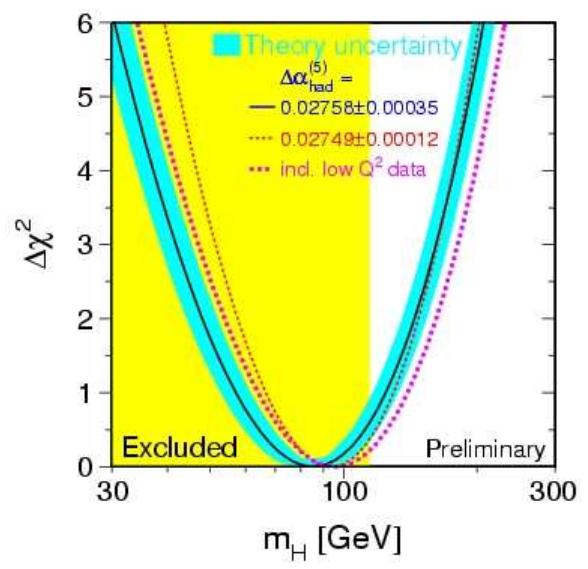

Figure 1.4: Indirect search for the Higgs boson: $\Delta \chi^{2}$ (black line) of the fit to the EW parameters (top quark and $W$ boson masses) as a function of the Higgs masses. The yellow band represents excluded Higgs masses at 95\% CL.

Tevatron Searches: Tevatron performed Higgs searches in proton-antiproton collisions at $\sqrt{s}=1.96 \mathrm{TeV}$. Both the D0 and CDF collaborations have analyzed $\sim 10.0 \mathrm{fb}^{-1}$ of data, studying, primarily, Higgs associated production $(Z H \rightarrow \nu \nu b \bar{b}, W H \rightarrow e \nu b \bar{b}, W H \rightarrow \mu \nu b \bar{b}$ 
and $\left.W H \rightarrow W W^{+} W^{-}\right)$and gluon fusion $(H \rightarrow W W)$. The 2012 combined limit can be seen in Fig. 1.5. The result excludes both 147 - $180 \mathrm{GeV}$ and $100-103 \mathrm{GeV}$ mass windows at $95 \%$ confidence level and a $2.5 \sigma$ excess of events was found between 115 and $135 \mathrm{GeV}$, indicating evidence of SM Higgs production at the Tevatron [9].

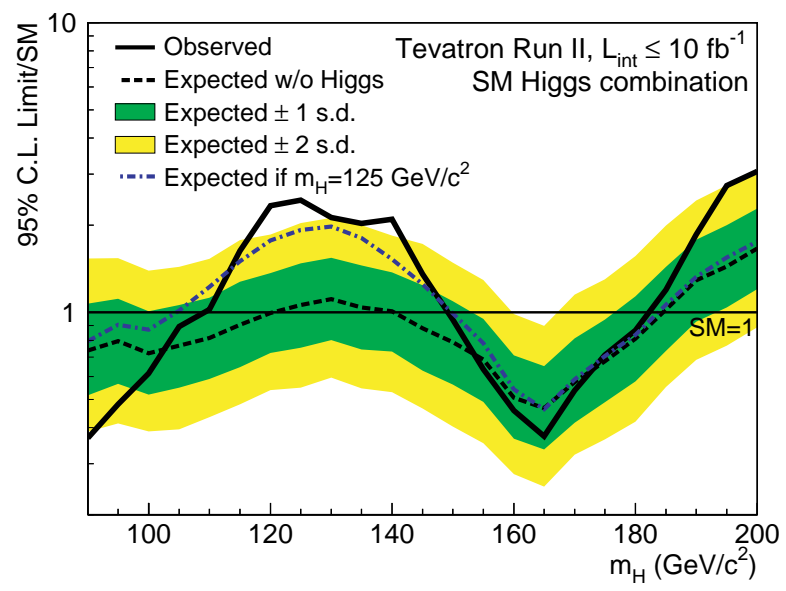

Figure 1.5: 2012 Tevatron limits on SM Higgs production. Mass regions of $147-180 \mathrm{GeV}$ and $100-103 \mathrm{GeV}$ has been excluded at $95 \%$ confidence level and a $2.5 \sigma$ evidence of production has been reported.

LHC Searches: Both CMS and ATLAS collaborations have searched for Higgs boson in proton-proton collisions at $\sqrt{s}=7$ and $8 \mathrm{TeV}$ respectively. The main Higgs production process studied were $H \rightarrow \gamma \gamma, H \rightarrow Z Z$, jets from weak vector boson fusion with $H \rightarrow \tau \tau$, $H \rightarrow \gamma \gamma$ and $H \rightarrow W W$.

In June 2012 both CMS and ATLAS reported the discovery of a particle with Higgs-like properties with a mass of $\sim 125 \mathrm{GeV}[10,11]$. CMS analyzed 5.1 and $5.3 \mathrm{fb}^{-1}$ of data at $\sqrt{s}=7$ and $8 \mathrm{TeV}$ respectively, and a particle decaying into $\gamma \gamma$ and $Z Z$ with mass a of $125.4 \pm 0.4$ (stat) \pm 0.5 (sys) was found with a $5.8 \sigma$ significance (Fig. 1.6). 


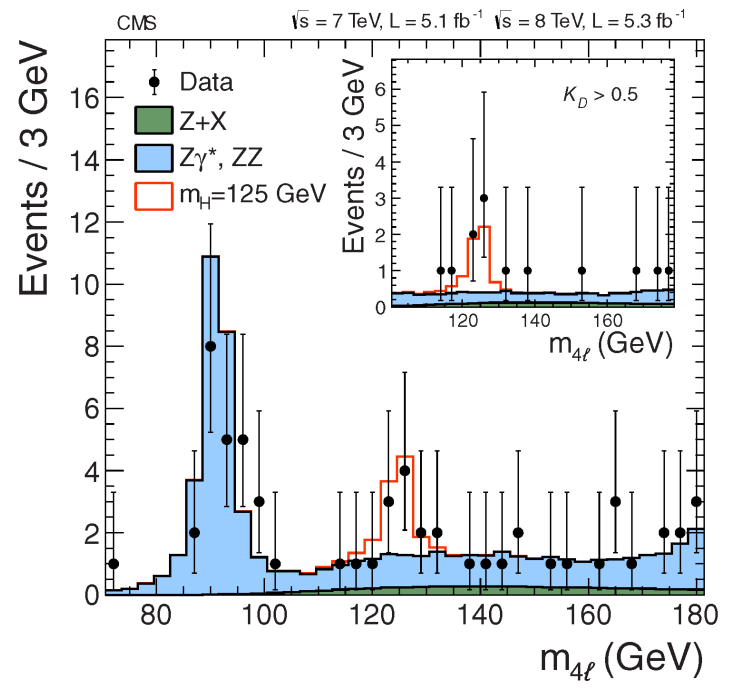

Figure 1.6: 2012 CMS Higgs production result in the $H \rightarrow Z Z \rightarrow 4$ lepton channel.

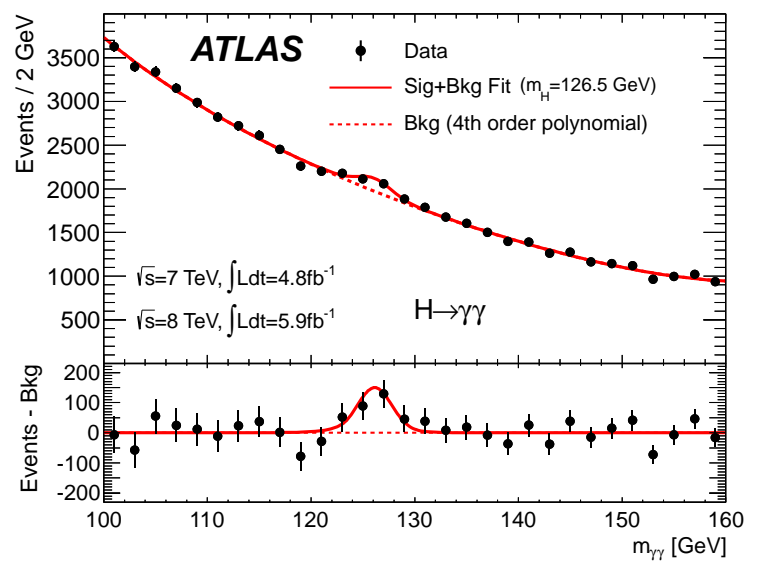

Figure 1.7: 2012 ATLAS Higgs production result in the $H \rightarrow \gamma \gamma$ channel. 
ATLAS analyzed 4.8 and $5.9 \mathrm{fb}^{-1}$ of data at $\sqrt{s}=7$ and $8 \mathrm{TeV}$ respectively, and a particle decaying into $\gamma \gamma$ and $Z Z$ with mass a of $126.0 \pm 0.4$ (stat) \pm 0.4 (sys) was found with a $5.9 \sigma$ significance (Fig. 1.7).

The goal of the present work is to present a SM Higgs boson search in both $g g \rightarrow H$ and $Z H$ channels. As we look for four isolated leptons in the final state we are interested in two processes, first $g g \rightarrow H \rightarrow Z Z^{*}($ or $Z Z) \rightarrow \ell^{+} \ell^{-} \ell^{\prime+} \ell^{\prime-}$, and second the $Z H$ associated production where $H \rightarrow \tau \tau \rightarrow \ell \nu \nu \ell \nu \nu, H \rightarrow W W \rightarrow \ell \nu \ell \nu$, and $H \rightarrow Z Z$ where at least one of the $Z$ bosons decays leptonically. This is in addition to the ones already studied at the Tevatron. The three charged leptonic decay channels that are studied in this analysis are:

- $Z Z \rightarrow$ eeee;

- $Z Z \rightarrow \mu \mu \mu \mu$

- $Z Z \rightarrow e е \mu \mu(\mu \mu e e)$

Also, besides $g g \rightarrow H$ and $Z H$, there are non Standard Model processes that have four leptons in the final state such as doubly charged Higgs $H^{++}[12], Z Z$ production from resonances [13], or $Z Z$ production in supersymmetry [14]. However, such processes are not in the scope of this work. 


\section{CHAPTER 2 THE TEVATRON COLLIDER AND THE DO DETECTOR}

This chapter describes the Tevatron accelerator at Fermilab. Until its recent shutdown the Tevatron collider complex at Fermi National Accelerator Laboratory was for over 20 years the highest energy hadron particle collider on the planet. It accelerated proton and antiproton beams to an energy of $0.98 \mathrm{TeV}$ each and collided them at two interaction points, B0 and D0. Two complex multi-purpose particle detectors (CDF and D0) located at these points studied the outcome of those collisions. The analysis presented in this dissertation was performed with the data collected by the D0 experiment.

\subsection{The Coordinate System}

The coordinate system adopted is the cylindrical $(\rho, \phi, z)$, with its origin matching the center of the detector, with the $z$ axis pointing in the same direction as the proton beam and the $y$ axis pointing to the top of the detector. Sometimes it is more convenient to use the spherical coordinate system, where the polar angle $\theta$ is the angle between the particles coming out from the collision and the direction of the beam and the azimuthal angle $\phi$ is measured in the plane perpendicular to the direction of the beam as shown in Fig 2.1.

The rapidity $y$ of a particle with energy $E$ and longitudinal momentum $p_{L}$ is defined as 


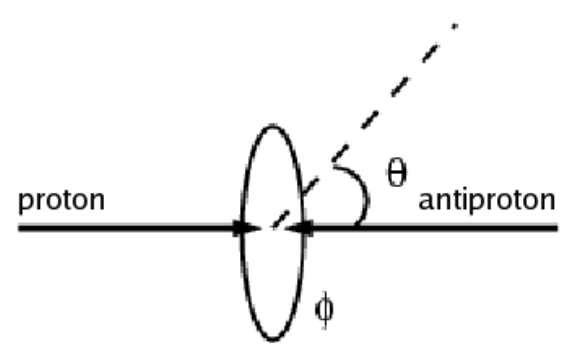

Figure 2.1: Coordinate System.

$$
y=\frac{1}{2} \log \left(\frac{E+p_{L}}{E-p_{L}}\right) .
$$

At the limit of very high energies, where the energy of a particle is much greater than its mass $(E \gg m)$, we have $y \approx \eta$, where $\eta$ is the pseudo-rapidity of the particle:

$$
\eta=-\ln \left|\tan \frac{\theta}{2}\right|
$$

The pseudo-rapidity is measured from a perpendicular direction with respect to the $p \bar{p}$ axis in the opposite direction to the angle $\theta$, and it is commonly used to indicate particular regions of the detector.

In high energy particle collisions, another important variable is the transverse momentum $p_{T}$, which is the projection of the vector momentum onto the plane perpendicular to the beam axis: 


$$
p_{T}=p \sin \theta
$$

The same reasoning allows us to define what is called the transverse energy $E_{T}$ of a particle:

$$
E_{T}=\sqrt{p_{T}^{2}+m^{2}}
$$

\subsection{Luminosity and Cross section}

The luminosity $\mathcal{L}$ is a quality factor of a collider reflecting the size and the density of the beam as well as the frequency of collisions, and it determines the observed rate of interactions of a specific type by knowing the cross section $\sigma$ of the process by

$$
\frac{\mathrm{d} N}{\mathrm{~d} t}=\sigma \mathcal{L}
$$

Luminosity (sometimes referred to as the instantaneous luminosity) can be expressed in terms of the number of protons and antiprotons in a bunch $\left(N_{p}\right)$, the number of bunches $\left(N_{B}\right)$, frequency of collisions $(f)$ and the beam size at the interaction point $\left(\sigma_{p}\right)$ as

$$
\mathcal{L}=\frac{f N_{B} N_{p} N_{\bar{p}}}{2 \pi\left(\sigma_{p}^{2}+\sigma_{\bar{p}}^{2}\right)} \mathcal{F}\left(\sigma_{l} / \beta^{*}\right)
$$

where $\mathcal{F}$ is a form factor depending on $\sigma_{l} / \beta^{*}$, the ratio of bunch length to the beta function at the interaction point. The integrated luminosity

$$
L=\int_{T} \mathcal{L} d t
$$


provides the total collider performance over a certain period of time.

The Tevatron collider is in fact the last stage in a chain of different accelerators (Fig. 2.2) which raises the proton's energy up until it reaches $150 \mathrm{GeV}$ before being sent into the Tevatron Ring:

\section{Accelerator Overview}

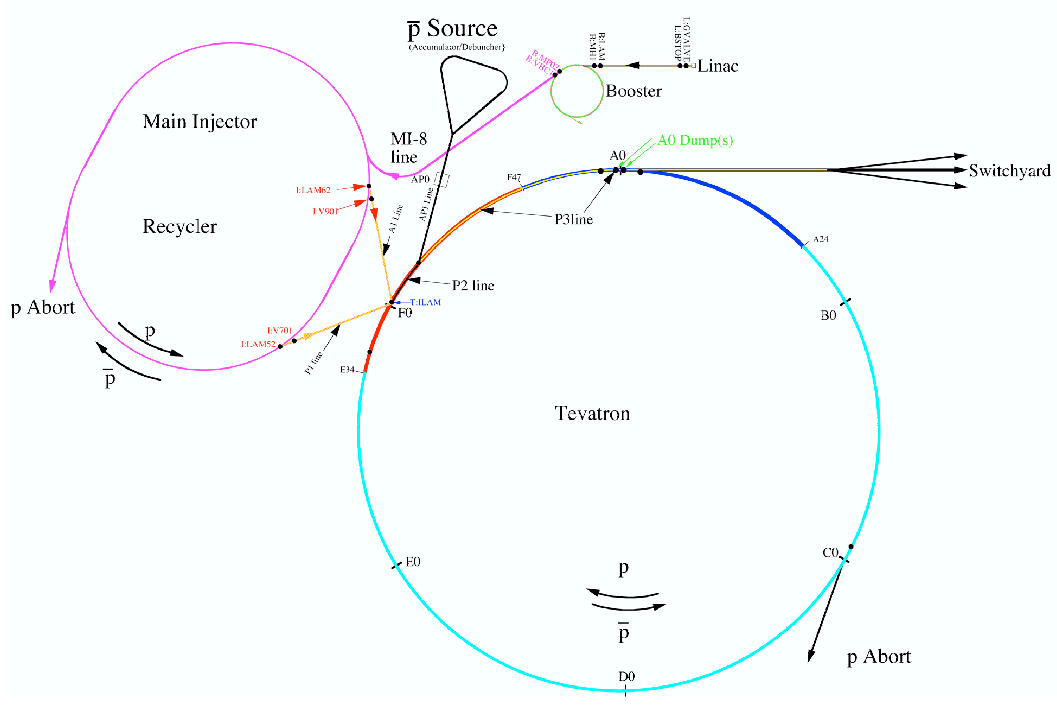

Figure 2.2: The Fermilab Collider Complex.

- Cockroft-Walton preaccelerator - accelerates hydrogen ions to $750 \mathrm{KeV}$.

- Linear Accelerator - accelerates ions to $400 \mathrm{MeV}$ and removes electrons.

- The Booster Synchrotron - accelerates protons to $8 \mathrm{GeV}$ and also supplies the antiproton Source with them.

- The Main Injector - accelerates protons and antiprotons to $150 \mathrm{GeV}$.

- Tevatron - accelerates protons and antiprotons to $0.98 \mathrm{TeV}$. 
The antiprotons are created in the Antiproton Source and then pass through the Debuncher and Accumulator before entering the Main Injector.

\subsection{Detector Overview}

The D0 detector (Fig. 2.3) is a general purpose experiment designed for the study of $p \bar{p}$ collisions at a center-of-mass energy of $1.96 \mathrm{TeV}$. It ran at the Fermilab Tevatron collider from 1992 to 2011 and it was designed for the study of final states containing electrons, muons, jets and neutrinos coming out of the $p \bar{p}$ collisions taking place in its center. The detector operated from 1992 through 1996 [15] and underwent an upgrade from 1996 to 2001 [16] for Run II of the accelerator, being modified to allow precise measurements of momentum, electric charge of particles, vertex tagging of b-jets and low $p_{T} B$ physics processes. The D0 detector is $13 \mathrm{~m}$ high, $12 \mathrm{~m}$ wide and $20 \mathrm{~m}$ long, weight 5500 tons and sits on a moving platform in the collision hall to allow its access. 


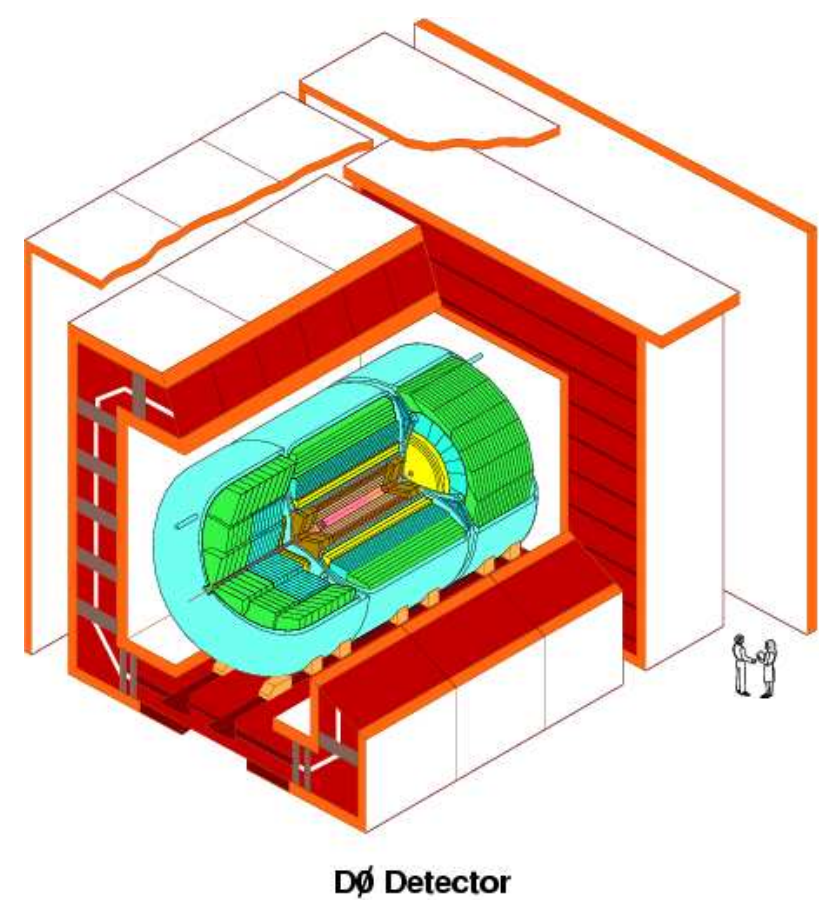

Figure 2.3: The D0 Detector [16].

The major subsystems of the D0 detector are:

- the Inner Tracking System

- the Calorimeter

- the Luminosity Monitor

- the Muon System

Fig. 2.4 shows a schematic view of the detector and highlights upgrades for RunII. 


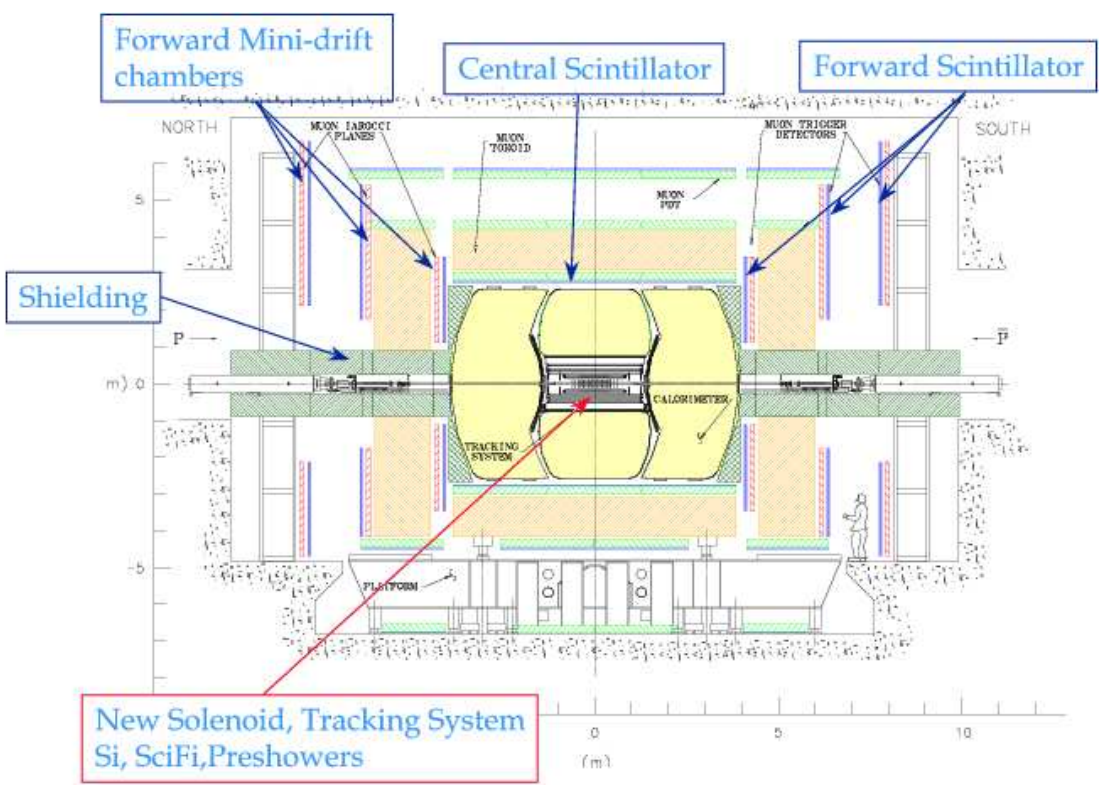

Figure 2.4: The D0 Detector highlighting upgrades for RunII [16].

\subsection{Inner Tracking System}

This is the innermost part of the detector and allows measurements of the paths of charged particles in a wide range of pseudorapidity. It consists of four subdetectors: the Silicon Microstrip Tracker (SMT), the Central Fiber Tracker (CFT), the Central Preshower (CPS) and the Forward Preshower (FPS). Both SMT and CFT are immersed in a $2 \mathrm{~T}$ magnetic field produced by the solenoid and are shown in Fig. 2.5.

The two tracking detectors locate the primary interaction vertex with a resolution of about $35 \mu \mathrm{m}$ along the beamline. They can tag $b$-quark jets with an impact parameter resolution of better than $15 \mu \mathrm{m}$ in $r-\phi$ for particles with transverse momentum $p_{T}>$ $10 \mathrm{GeV}$ at $|\eta|=0$. The high resolution of the vertex position allows good measurement of lepton $p_{T}$, jet transverse energy $\left(E_{T}\right)$, and missing transverse energy $\mathbb{E}_{T}$. For instance, 


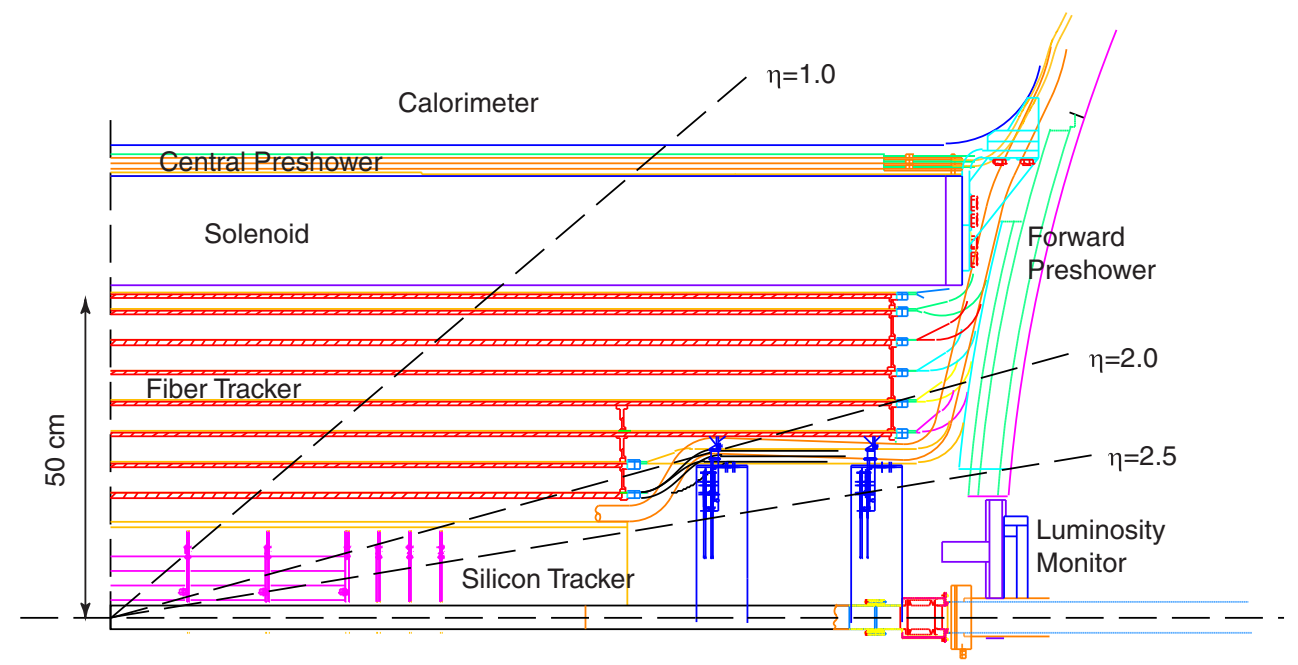

Figure 2.5: $r-z$ view of the tracking system [16].

at $|\eta|=0$, momentum resolutions are $2 \%, 5 \%$ and $17 \%$ for $p_{T}=, 1,10$ and $100 \mathrm{GeV}$ respectively [16].

\subsubsection{Silicon Microstrip Tracker}

The Silicon Microstrip Tracker [17] is the closest subdetector to the beam line. It has a total of 912 readout modules, with 792,576 channels and consists of six concentric barrel modules of 4 silicon layers with insertions of F-disks modules of 12 doublesided wedge detectors, and with two large-diameter H-disks with 24 wedges with two back-to-back single-sided sensors. There are 144 F-wedges and 96 full H-wedges in the tracker. The centers of the H-disks are located at $|z|=100.4,121.0 \mathrm{~cm}$; the F-disks are at $|z|=12.5,25.3,38.2,43.1,48.1$, and $53.1 \mathrm{~cm}$. The centers of the barrels are at $|z|=6.2,19.0,31.8 \mathrm{~cm}$. 
The (shown in Fig. 2.6) provides information about primary vertex with a resolution of $35 \mu \mathrm{m}$ along the beamline and b-jets tagging with an impact parameter resolution of about $15 \mu \mathrm{m}$ in the plane transverse to the beamline.

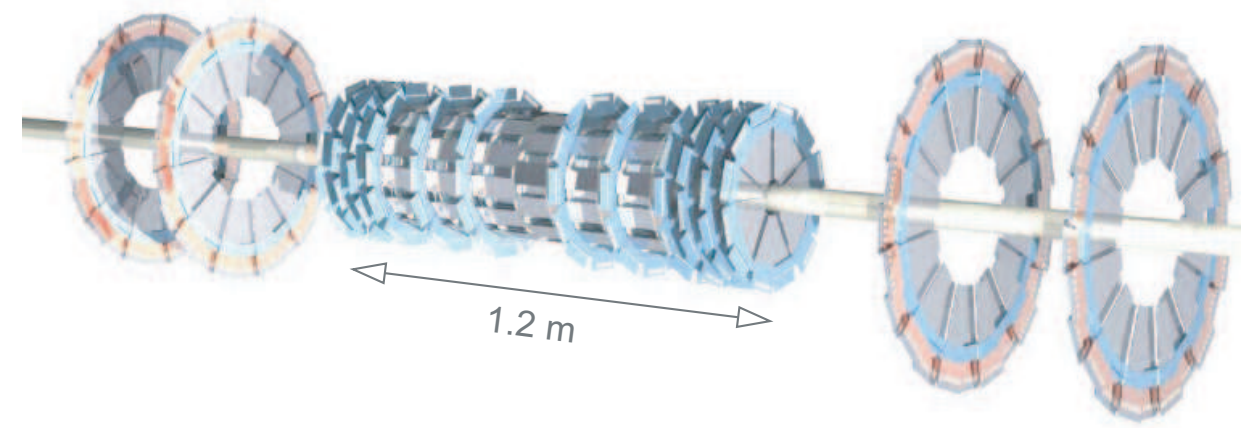

Figure 2.6: The DØ Silicon Microstrip Tracker [17].

\subsubsection{Central Fiber Tracker}

The Central Fiber Tracker is based on scintillating fiber technology with visible light photon counter (VLPC) readout [18], which work in an avalanche mode at $9 \mathrm{~K}$. With a total of 77,000 channels the CFT has 8 layers of fiber doublets, with each one consisting of two layers of $830 \mu \mathrm{m}$ diameter fibers with $870 \mu \mathrm{m}$ spacing, offset by half the fiber spacing. The CFT allows position measurements with a resolution of about $100 \mu \mathrm{m}$.

\subsubsection{Preshower detectors}

Both central and forward preshower detectors (CPS and FPS) (Fig. 2.7) play two roles: they measure the energy deposition of charged particles moving towards the calorimeters 
and the same time track such particles. This allows for the separation of electrons and photons. The CPS consists of 3 cylindrical layers with each one consisting of an array of triangular scintillating fibers equipped with wavelenght shifter fibers (WLS) with a total of 7000 channels. The FPS consists of 2 MIP and 2 shower layers with 15000 channels.

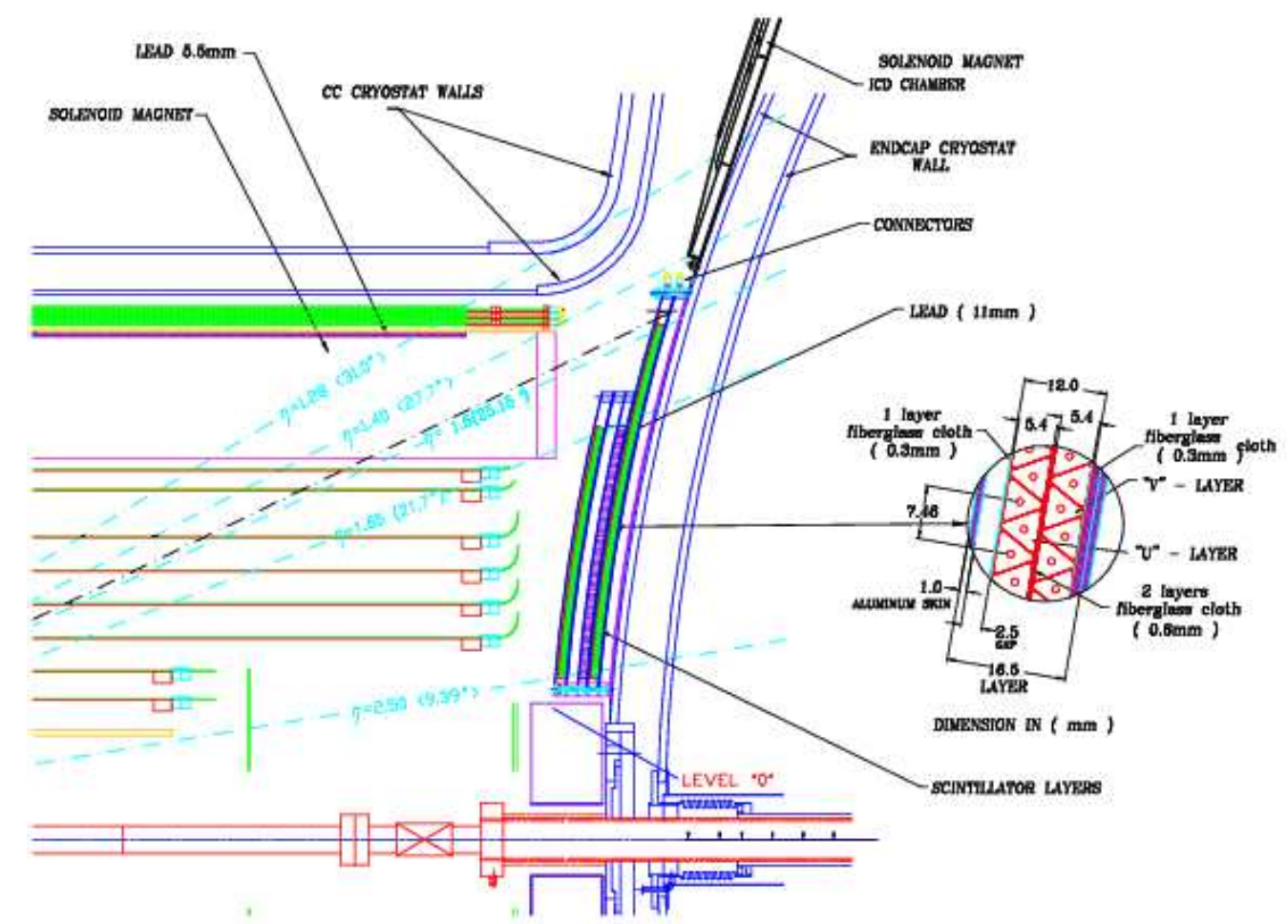

Figure 2.7: The central and forward preshower detectors. [16]

\subsection{Calorimeter}

A calorimeter is a device used to measure the energy deposited by a particle or a cluster (jet) of particles by absorption. When a particle interacts with the material of the calorimeter it generates a cascade of other particles which itself depends on the original 
particle's initial energy. There are two different kinds of calorimeters depending on if the incident particle generates an electromagnetic or a hadronic shower. Each kind is designed to maximize the rejection of the other type of shower.

The D0 calorimetry system consists of a sampling calorimeter which uses uranium as the absorbing material and liquid-argon as the ionizing material (as shown in Fig. 2.8) and an intercryostat detector.

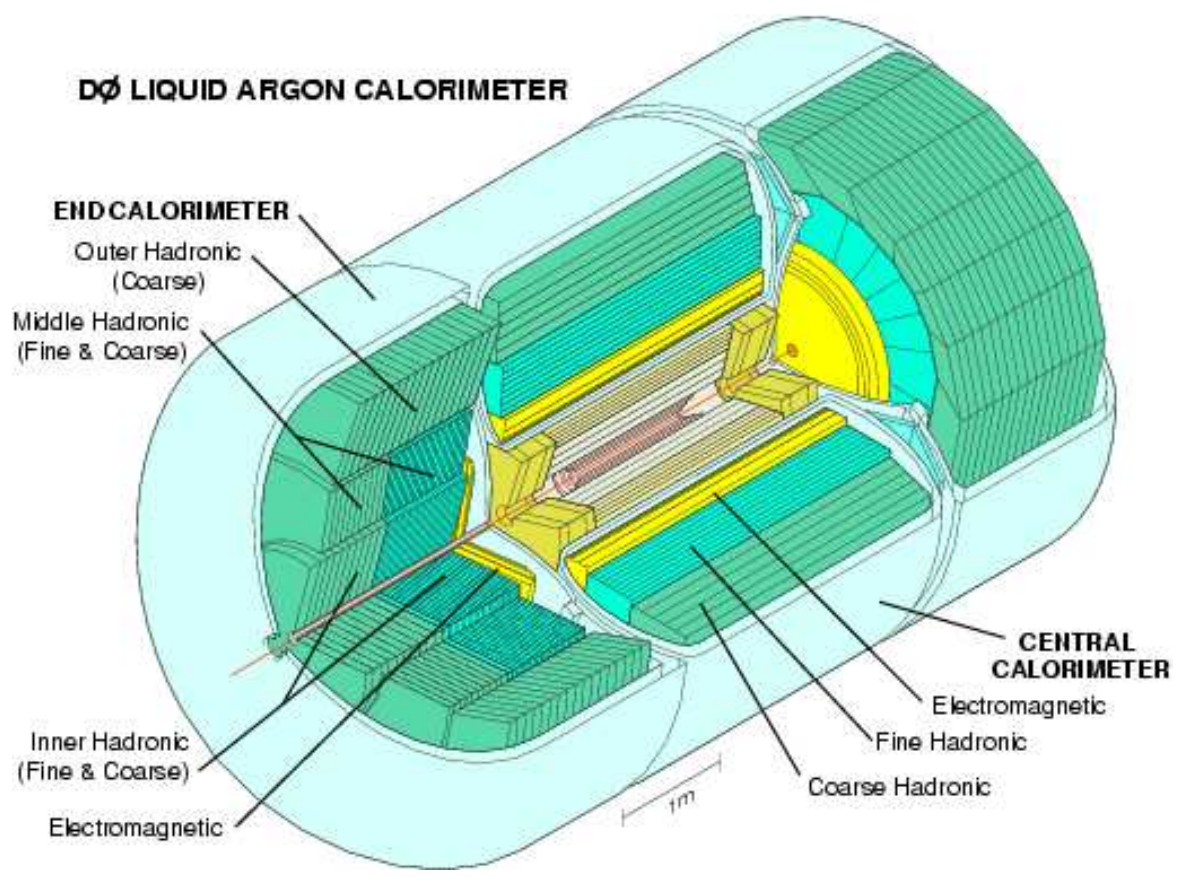

Figure 2.8: The D0 Calorimeter [17].

As shown in Fig. 2.9, the central calorimeter (CC) covers $|\eta|<1.2$ and the two end calorimeters, ECN (north) and ECS (south), extend coverage to $|\eta| \approx 4$. Each calorimeter contains an electromagnetic section closest to the interaction region followed by fine and coarse hadronic sections. The calorimeter is divided in modules, each one with cells containing both absorbing material and signal detectors. Each of these cells contains uranium plates for absorption and signal production through signal boards. The $2.3 \mathrm{~mm}$ gap 


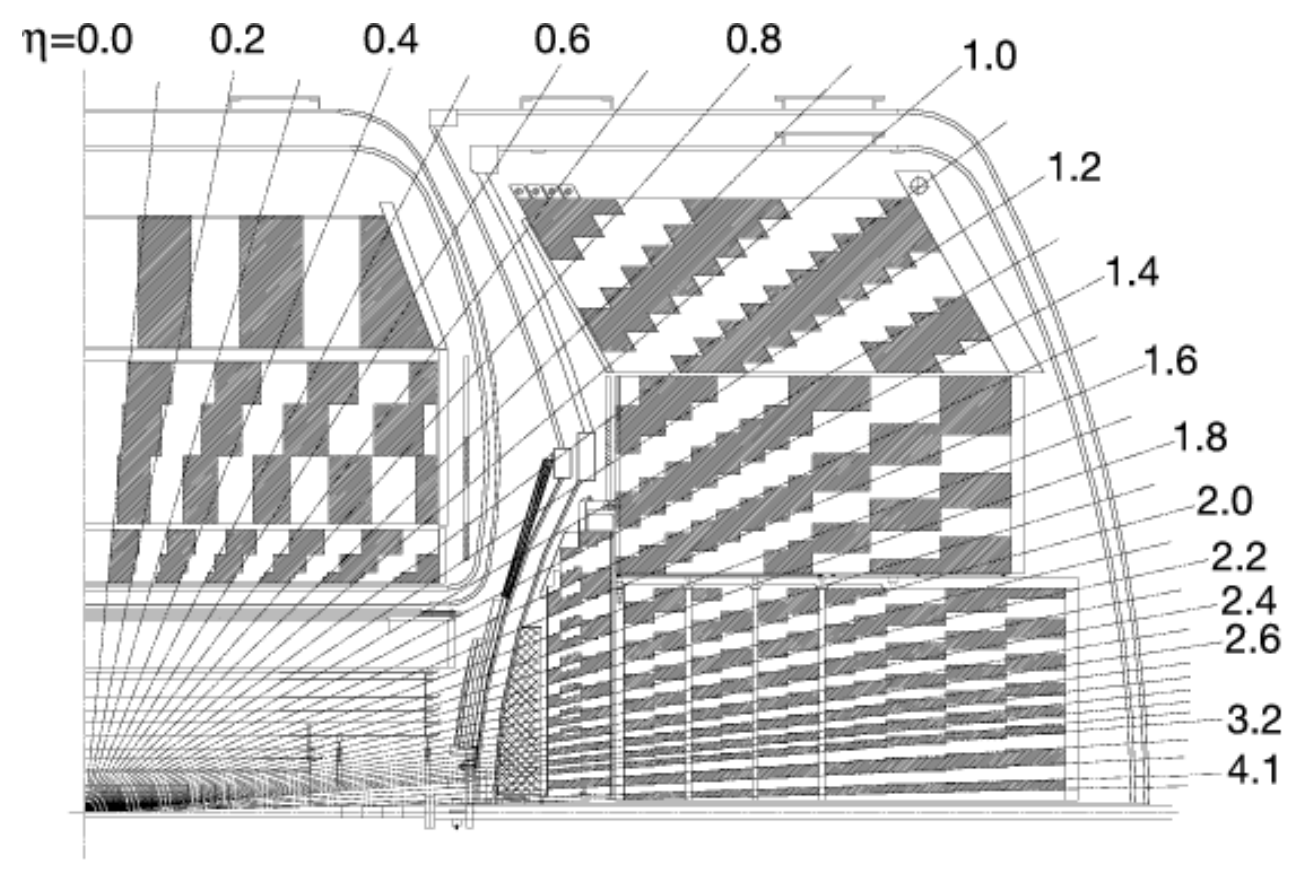

Figure 2.9: Schematic view of a portion of the D0 calorimeters showing the transverse and longitudinal segmentation pattern [17].

between each plate is filled with liquid argon. The signal system consists of signal boards made of two 0.5 mm-layers of insulating material G10. The external surface of the signal board is covered with resistive epoxy. During operation a tension of $2.0-2.5 \mathrm{kV}$ is applied to the resistive surfaces while the uranium plates remain grounded. The signal occurs when charged particles reach the liquid argon and produce ionization tracks, thus the released electrons are collected by the signal boards after a drift time of order of $450 \mathrm{~ns}$.

The CC covers the psedorapidity region of $|\eta|<1.2$ and consists of three cylindrical parts of modules. The electromagnetic part has four layers of cells. The first two measure the longitudinal development of the shower until around $2 \mathrm{X}_{0}{ }^{1}$. The third measures the shower until $7 \mathrm{X}_{0}$. The last layer goes until the maximum of $10 \mathrm{X}_{0}$. Each calorimeter cell

\footnotetext{
${ }^{1} \mathrm{X}_{0}$ is the radiation length of the material.
} 
has dimensions of $\Delta \eta \times \Delta \phi=0.1 \times 0.1$ except the third layer with $\Delta \eta \times \Delta \phi=0.05 \times 0.05$. The hadronic section of the calorimeter has a length of $7-9 \lambda^{2}$ and is divided in four layers of cells again with dimensions of $\Delta \eta \times \Delta \phi=0.1 \times 0.1$. The hadronic part is itself divided in two modules: 16 fine hadronic $(\mathrm{FH})$ that measure hadronic showers and 16 coarse hadronic $(\mathrm{CH})$ that measures any flow of energy escaping from the FH. The FH module covers the first three layers (of lengths of $0.9 \lambda, 1.0 \lambda, 1.3 \lambda$ respectively) of the four of the hadronic section. The FH cells have $6 \mathrm{~mm}$-thick plates of uranium-niobium while the $\mathrm{CH}$ consists of a single layer of length of $3.2 \lambda$ of cooper and iron absorbing plates of $46.5 \mathrm{~mm}$ of thickness.

The ends of the calorimeter cover a region of $1.3<|\eta|<4.2$. Each end calorimeter consists of three concentrical modules. However their electromagnetic part is a disk-shaped detector towards the innermost part of the hadronic calorimeter. The difference between it and its counterpart in $\mathrm{CC}$ is that it measures showers developing until $2.3 \mathrm{X}_{0}$ instead $2 \mathrm{X}_{0}$. The hadronic part of the EC's is divided into an internal part, consisting of $\mathrm{FH}$ and $\mathrm{CH}$ modules, an intermediate part with both $\mathrm{FH}$ and $\mathrm{CH}$ modules and an external part with only $\mathrm{CH}$ modules. All cells have a $\Delta \eta \times \Delta \phi=0.1 \times 0.1$ transverse segmentation. Fig. 2.10 shows how a typical cell.

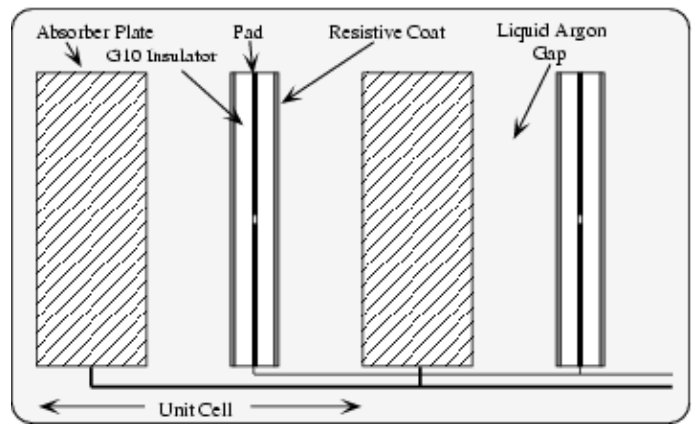

Figure 2.10: Calorimeter cell [17].

\footnotetext{
${ }^{2} \lambda$ is the nuclear interaction length of the material.
} 


\subsubsection{ICD and Massless Gaps}

As can be seen in Fig. 2.9, the region $0.8 \leq|\eta| \leq 1.4$ contains a large amount of material, not covered by the standard calorimeter instrumentation. This region is generically called intercryostat region (ICR) and includes the cryostat walls, module endplates and such. Rather than leaving gaps in the calorimeter, an additional detector component was placed in each of those two regions. These two scintillation counter arrays are named the intercryostat detectors or ICDs. Each ICD consists of 192 scintillator tiles of size $\Delta \eta=\Delta \phi=0.1$, which exactly matches the size of the regular calorimeter cells. The light output of the ICD tiles is collected by $1.3 \mathrm{~cm}$ diameter phototubes and sent over to the calorimeter electronics just as the liquid argon channels are.

In addition to ICDs there are two special regions in the calorimeter system - the socalled "Massless Gaps." They are special in that these channels have only the liquid argon and not the depleted uranium absorber plates. There are 320 such channels in CC and 192 in EC.

\subsection{Luminosity Monitor}

The Luminosity Monitor (LM) [19] is located about $135 \mathrm{~cm}$ away, in both directions, from the central interaction point of the D0 and made of 24 scintillator counters which covers the region of $2.7<|\eta|<4.4$. It measures the luminosity delivered by the Tevatron collider to D0 using the observed average number of inelastic collisions per beam crossing

$N^{\text {inel }}$ and the effective inelastic cross section $\sigma_{\text {eff }}^{\text {inel }}$ according to $\mathcal{L}=f \frac{N^{\text {inel }}}{\sigma_{\text {eff }}^{\text {inel }}}$ where $f$ is the beam crossing frequency [19]. 


\subsection{The Muon System}

Muons have sufficiently large lifetime to pass through the calorimeter depositing little energy in it. A dedicated muon detection system is needed in addition to the calorimeter.

For muon triggering and measurement, the upgraded $\mathrm{D} \varnothing$ detector uses central muon system proportional drift tubes (PDTs) and toroidal magnets, central scintillation counters and a forward muon system [20]. The central muon system provides coverage for $|\eta| \leq 1.0$. The forward muon system extends muon detection to $|\eta| \approx 2.0$. It uses mini drift tubes ( MDTs) rather than PDTs, and includes trigger scintillation counters and beam pipe shielding.

\subsubsection{Central muon detector}

The central muon system consists of a $1.8 \mathrm{~T}$ toroidal magnet, drift chambers, the cosmic cap and bottom scintillation counters, and the $A \phi$ scintillation counters. The central toroid is a square annulus $109 \mathrm{~cm}$ thick whose inner surface is about $318 \mathrm{~cm}$ from the Tevatron beamline; it covers the region $|\eta|<1$. It provides a stand-alone muon-system momentum measurement, which $i$ ) enables a low- $p_{T}$ cutoff in the Level 1 muon trigger, $\left.i i\right)$ allows for cleaner matching with central detector tracks, iii) rejects $\pi / K$ decays.

The three layers of drift chambers are located inside (A layer) and outside (B and C layers) of the central toroidal magnet. Approximately 55\% of the central region is covered by three layers of PDTs; close to $90 \%$ is covered by at least two layers (Fig. 2.11). The drift chambers are large, typically $2.8 \times 5.6 \mathrm{~m}^{2}$, and made of rectangular extruded aluminum tubes. The PDTs outside of the magnet have three decks of drift cells, while the A layer 
has four decks, with the exception of the bottom A-layer PDTs which have three decks. The cells are $10.1 \mathrm{~cm}$ across; typical chambers are 24 cells wide and contain 72 or 96 cells.

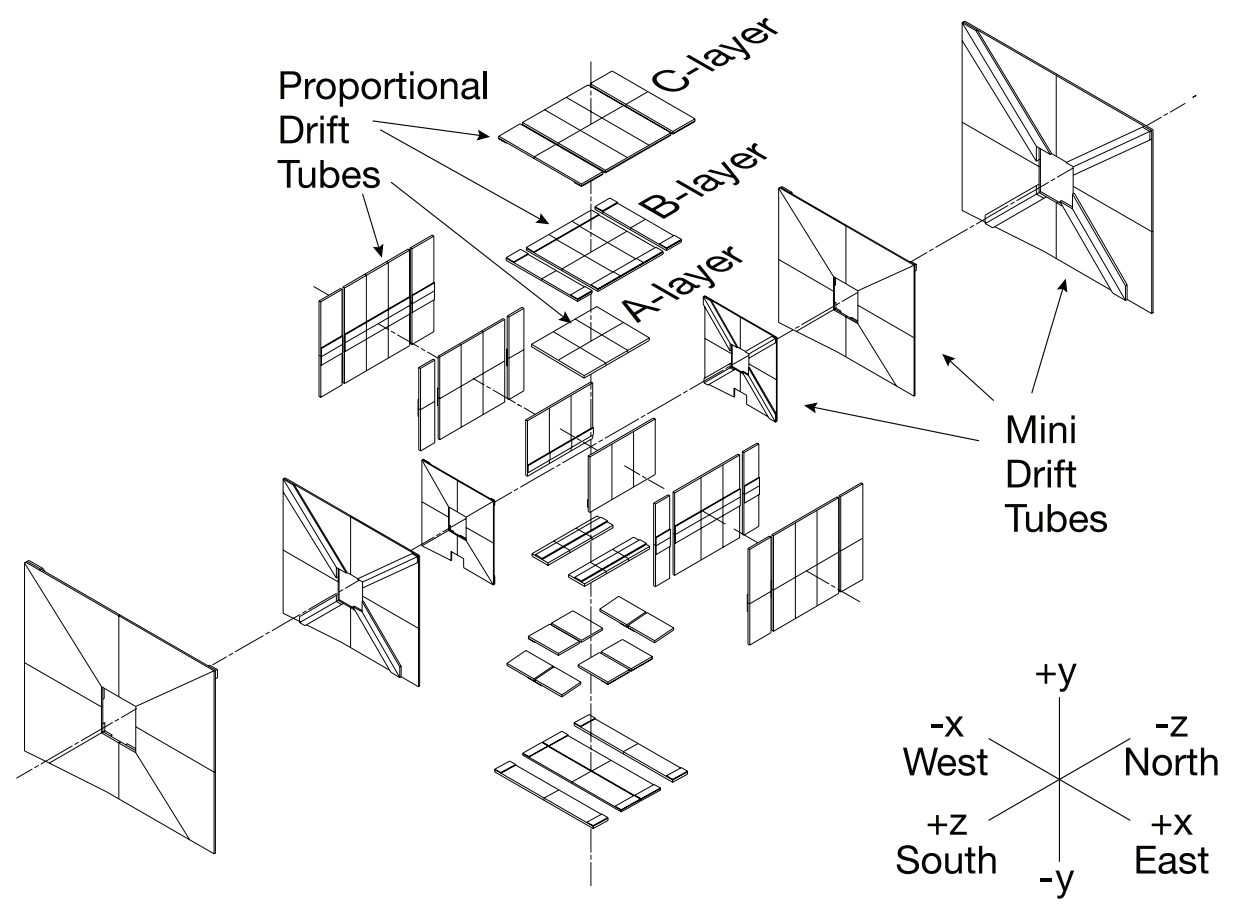

Figure 2.11: The DØ Muon System (PDT + MDT) [20].

The $A \phi$ scintillation counters cover in $\phi$ the A-layer PDTs, those between the calorimeter and the toroid. They provide a fast detector for triggering on and identifying muons and for rejecting out-of-time backscatter from the forward direction. Fig. 2.12 demonstrates the layout of these counters as well as the forward, cosmic cap, and bottom scintillation counters. All of them are used primarily for fast triggering and background rejection. 


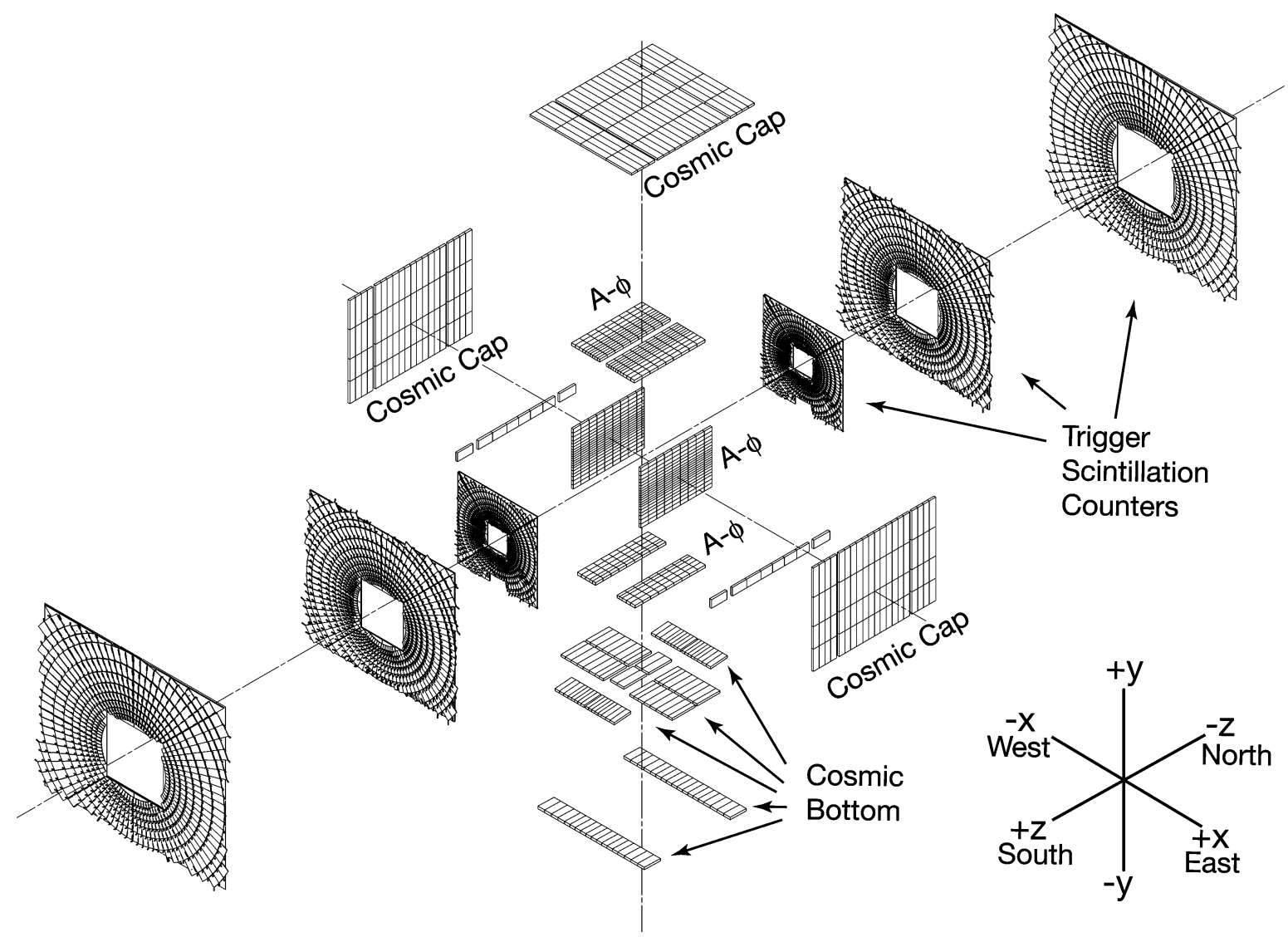

Figure 2.12: The DØ Muon System (Scintillators) [17].

\subsubsection{Forward muon detector}

The forward muon detector system covers $1.0 \leq|\eta| \leq 2.0$ and consists of four major parts: the end toroidal magnets, three layers of MDTs for muon track reconstruction, three layers of scintillation counters for triggering on events with muons, and shielding around the beam pipe. MDTs were chosen for their short electron drift time (below $132 \mathrm{ns),} \mathrm{good}$ coordinate resolution (less than $1 \mathrm{~mm}$ ), radiation hardness, high segmentation, and low occupancy. The MDTs are arranged in three layers (A, B, and C, with A closest to the interaction region inside the toroidal magnet and $\mathrm{C}$ furthest away), each of which is divided 
into eight octants, as illustrated in Fig. 2.11. Each layer consists of three (layers B and C) or four (layer A) planes of tubes mounted along magnetic field lines. The entire MDT system contains 48,640 wires. The momentum resolution of the forward muon system is approximately $20 \%$ for muon momentum below $40 \mathrm{GeV}$. The overall muon momentum resolution is determined by the central tracking system for muons with momentum up to approximately $100 \mathrm{GeV}$.

\subsection{The Do Trigger System}

Proton-antiproton collisions at the center of the detector produce data at a rate of $\approx 2$ MHz. Due to the impossiblity to record all this data a trigger system is designed to reduce such rate and at the same time accept candidate events to the various physics analysis. In order to perform this task with maximum efficiency the D0 trigger system consists of a 3-level structure: Level 1 or L1, Level 2 or L2, and Level 3 or L3, with each succeeding level examining fewer events but in greater detail and with more complexity. L1 comprises a collection of hardware trigger elements that provide a trigger accept rate of about $2 \mathrm{kHz}$. In L2 hardware engines and embedded microprocessors associated with specific subdetectors provide information to a global processor to construct a trigger decision based on individual objects as well as object correlations. The L2 system reduces the trigger rate by a factor of about two and has an accept rate of approximately $1 \mathrm{kHz}$. Candidates passed by L1 and L2 are sent to a farm of L3 microprocessors where sophisticated algorithms reduce the rate to about $50 \mathrm{~Hz}$. These events are recorded for offline reconstruction. An overview of the $\mathrm{D} \varnothing$ trigger and data acquisition system is shown in Fig. 2.13. A block diagram of the L1 and L2 trigger systems is shown in Fig. 2.14. 


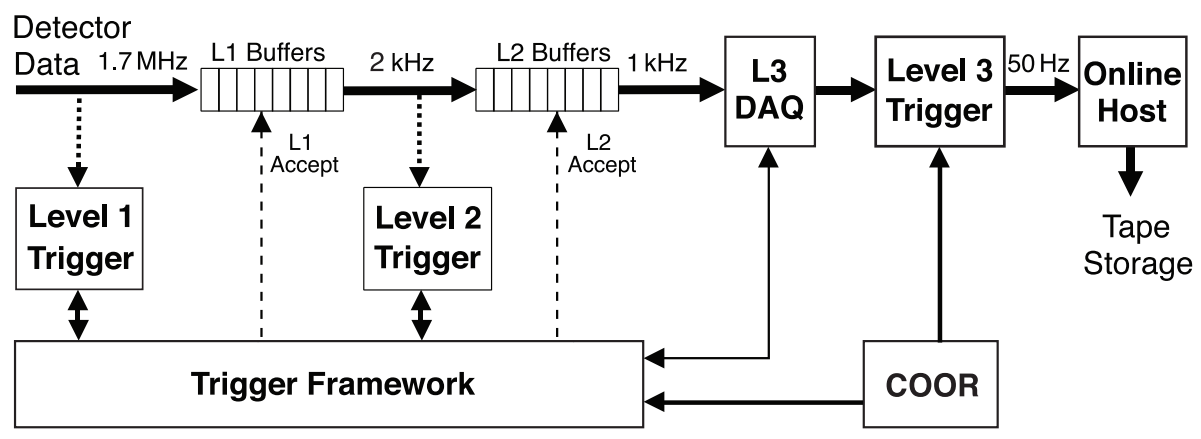

Figure 2.13: Overview of the D $\varnothing$ trigger and data acquisition systems [17].

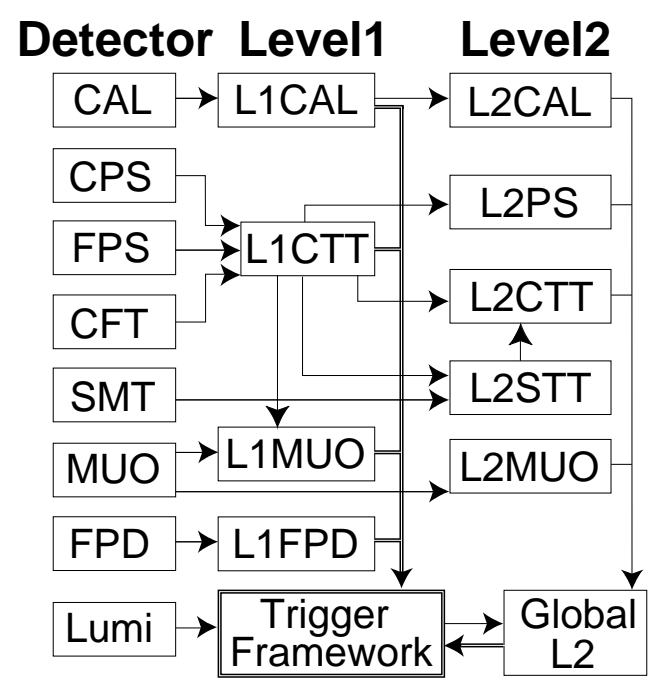

Figure 2.14: Block diagram of the D $\varnothing$ L1 and L2 trigger systems. The arrows show the flow of trigger-related data [17]. 


\section{CHAPTER 3}

\section{DATA AND MONTE CARLO SAMPLES, TRIGGER AND LUMINOSITY}

The next sections give details of the data and MC samples, triggers and total luminosity utilized for this analysis.

\subsection{Data Samples}

The analysis uses the full RunIIa and RunIIb (RunIIb is itself divided into RunIIb1, b2, b3 and b4) datasets collected with the D0 detector from the period of 2001 through September 30, 2011 consisting of runs in the range 151817-275727. We use the certified samples [21] for the analysis. Below, CSG_CAF_2EMhighpt denotes a certified skimmed data sample where at least 2 electrons with $p_{T}>5.0 \mathrm{GeV}$ are selected. CSG_CAF_2MUhighpt denotes a certified skimmed data sample where at least 2 muons with $p_{T}>5.0 \mathrm{GeV}$ are selected. Other terms in the definitions refer to the version of the reconstruction software.

The eeee channel uses the following sample definition:

- Run IIa

- CSG_CAF_2EMhighpt_PASS3_p18.14.00

- Run IIb1 
- CSG_CAF_2EMhighpt_PASS2_p21.10.00

- Run IIb2

- CSG_CAF_2EMhighpt_PASS4_p21.10.00_p20.12.00

- CSG_CAF_2EMhighpt_PASS4_p21.10.00_p20.12.01

- CSG_CAF_2EMhighpt_PASS4_p21.10.00_p20.12.02

- CSG_CAF_2EMhighpt_PASS4_p21.10.00_p20.12.04

- CSG_CAF_2EMhighpt_PASS4_p21.12.00_p20.12.05_allfix

- Run IIb3

- CSG_CAF_2EMhighpt_PASS5_p21.18.00_p20.16.07_fix

- CSG_CAF_2EMhighpt_PASS5_p21.18.00_p20.16.07_reduced2

- CSG_CAF_2EMhighpt_PASS5_p21.18.00_p20.16.08

- Run IIb4

- CSG_CAF_2EMhighpt_PASS6_p21.20.00_p20.18.02b

- CSG_CAF_2EMhighpt_PASS6_p21.20.00_p20.18.02b_fix

- CSG_CAF_2EMhighpt_PASS6_p21.21.00_p20.18.03

- CSG_CAF_2EMhighpt_PASS6_p21.22.00_p20.18.04

- CSG_CAF_2EMhighpt_PASS6_p21.22.00_p20.18.05

For $е e \mu \mu$ and $\mu \mu \mu \mu$ channels we use the following sample definition:

- Run IIa

- CSG_CAF_2MUhighpt_PASS3_p18.14.00 
- Run IIb1

- CSG_CAF_2MUhighpt_PASS2_p21.10.00

- Run IIb2

- CSG_CAF_2MUhighpt_PASS4_p21.10.00_p20.12.00

- CSG_CAF_2MUhighpt_PASS4_p21.10.00_p20.12.01

- CSG_CAF_2MUhighpt_PASS4_p21.10.00_p20.12.02

- CSG_CAF_2MUhighpt_PASS4_p21.10.00_p20.12.04

- CSG_CAF_2MUhighpt_PASS4_p21.12.00_p20.12.05_allfix

- Run IIb3

- CSG_CAF_2MUhighpt_PASS5_p21.18.00_p20.16.07_fix

- CSG_CAF_2MUhighpt_PASS5_p21.18.00_p20.16.07_reduced2

- CSG_CAF_2MUhighpt_PASS5_p21.18.00_p20.16.08

- Run IIb4

- CSG_CAF_2MUhighpt_PASS6_p21.20.00_p20.18.02b

- CSG_CAF_2MUhighpt_PASS6_p21.20.00_p20.18.02b_fix

- CSG_CAF_2MUhighpt_PASS6_p21.21.00_p20.18.03

- CSG_CAF_2MUhighpt_PASS6_p21.22.00_p20.18.04

- CSG_CAF_2MUhighpt_PASS6_p21.22.00_p20.18.05

Because this analysis looks for one of the smallest cross-section processes in the SM, which also has very little background, we attempt to apply very loose kinematical selections. The idea is that if an event is contained in one of the above samples, we are interested in 
analyzing it. The same can be said for trigger selection. Trigger efficiency can be calculated by dividing the number of events that pass all the selection criteria (see chapter 5) plus applied triggers divided by the number of events that pass all the selection criteria only. In most analysis it is commom to include trigger selection as part of the selection cuts applied. However, here we opted for keep them apart in order to be more specific when discussing the probality of event to pass the triggers. Based on the characteristics of our signal we applied single muon, dimuon, single electron and dielectron triggers. In all cases we have a trigger efficiency for our signal close to $100 \%$. A couple of examples are in order to help to show that. For instance, when calculating the trigger efficiency for four muons we have to take into account either a failure in an A-PDT or their limited coverage giving a trigger efficiency of zero and the particular topology of the event. For instance, all nseg = 3 muons pass a muon trigger, all nseg $=0$ fail and so does most of nseg $=1,2$. In the case of muons in the covered A-PDT region the probability of a nseg $=3$ muon to pass muon triggers is $\approx 75 \%$, thus the probability of all four muons pass the trigger is $1-(1-0.75)^{4}$ $=0.996 ;$ in the case of 3 muons failing and one passing we have $1-4 \times 0.75 \times(1-0.75)^{3}$ $=0.953$. A conservative probability of an electron in the EC region of the detector to fire an electron trigger is $\approx 80 \%$. Therefore, in the case of two EC electrons passing we have $1-(1-0.80)^{2}=960 \%$. Thus, in both cases we obtain probalities close to but not exactly $100 \%$.

\subsection{Luminosity}

The integrated luminosity for each channel separately is determined via an unprescaled trigger from the trigger list that covers the full data range after applying full data quality corrections. These corrections are applied to ensure that particular events flagged as "bad" 
during data collection periods do not enter the final sample used for various analysis. The integrated luminosity for the eеeе, $\mu \mu \mu \mu$ and $е е \mu \mu$ are respectively 9.8, 9.6 and $9.6 \mathrm{fb}^{-1}$. Table 3.1 shows RunIIa and RunIIb luminosities when data quality corrections are not applied to each channel separately. A luminosity uncertainty of $6.1 \%$ is assigned and determined by the D0 luminosity group [19].

Table 3.1: Table of luminosities used in this analysis. The error is the $6.1 \%$ standard luminosity uncertainty.

\begin{tabular}{c|c}
\hline \hline Run Epoch & Integrated Luminosity $\left(\mathrm{pb}^{-1}\right)$ \\
\hline RunIIa & $1244 \pm 53$ \\
\hline RunIIb & $9200 \pm 396$ \\
\hline Total & $10444 \pm 449$ \\
\hline \hline
\end{tabular}

\subsection{Monte Carlo Samples}

We use PYTHIA [22] MC for simulation of $g g \rightarrow H$ and $Z H$ production. The samples were processed through standard D0 simulation code (DOSim) followed by processing through the full RunIIa and RunIIb geometry detector simulation (d0gstar) with zerosuppressed zero-bias data overlaid. These samples are then reconstructed using proper algorithms depending if they are RunIIa or RunIIb samples.

In addition to the $g g \rightarrow H \rightarrow Z Z \rightarrow \ell^{+} \ell^{-} \ell^{\prime+} \ell^{\prime-}, Z H$ production mode contributes primarily through decays of $H \rightarrow \tau \tau \rightarrow \ell \nu \nu \ell \nu \nu, H \rightarrow W W \rightarrow \ell \nu \ell \nu$, and $H \rightarrow Z Z$, where at least one of the $Z$ bosons decays leptonically. For a Higgs boson mass of $125 \mathrm{GeV}$, for instance, the branching fraction (BF) of $H \rightarrow Z Z \rightarrow \ell^{+} \ell^{-} \ell^{\prime+} \ell^{\prime-}$ is $2.67 \%$, while for the same mass $H \rightarrow \tau \tau \rightarrow \ell \nu \nu \ell \nu \nu$ is $2.23 \%, H \rightarrow W W \rightarrow \ell \nu \ell \nu$ is $21.6 \%$. All Higgs boson production cross sections and branching fractions are taken from [23]. 
The MC samples are reweighted at an event level by the luminosity profile as determined by comparing the profile from the appropriate data sample to the profile from the zerobias overlay in the MC. In addition, the MC has to be reweighted for the $z$ coordinate of the primary vertex which is randomly distributed by a Gaussian centered at 0 with a width of $25 \mathrm{~cm}$ in the MC generation, which is quite different from the longitudinal shape of the luminous region in the data. The correction uses a fit to the beamshape region out to 60 $\mathrm{cm}$. The reweighting uses the method from note [24].

The MC samples are additionally reweighted on a lepton by lepton basis by a scale factor as the selection efficiency of a lepton is not the same for data and MC. This data/MC scale factor is determined by dividing data efficiency by MC efficiency as determined by the corresponding EM [29] [25] and Muon [27] ID groups. More details on the specific correction factors is discussed in sections 4.1 and 4.2. This is applied to each lepton, and the product for all leptons gives an event weight which is combined with the previously determined weights.

In addition to reweighting, we apply additional lepton momentum smearing to MC events. This is done to achieve better momentum matching between data and MC. The added smearing is determined by studies of $J / \Psi$ and $Z \rightarrow \mu \mu$ decays [27]. Details on both $g g \rightarrow H$ and $Z H$ signal samples used for both RunIIa and RunIIb are given in Tables 3.2 through 3.5. 
Table 3.2: RunIIa Pythia Higgs $g g \rightarrow H$ MC samples used in this analysis.

\begin{tabular}{lcc}
\hline Higgs mass $(\mathrm{GeV})$ & $\sigma \times \mathrm{BF}(\mathrm{pb})$ & Events \\
\hline$m_{H}=115$ & 0.000108226 & 95719 \\
$m_{H}=120$ & 0.000174926 & 96548 \\
$m_{H}=125$ & 0.000258425 & 96686 \\
$m_{H}=130$ & 0.000345479 & 94958 \\
$m_{H}=135$ & 0.000421789 & 96384 \\
$m_{H}=140$ & 0.000473139 & 96092 \\
$m_{H}=145$ & 0.000487436 & 96534 \\
$m_{H}=150$ & 0.000455113 & 97402 \\
$m_{H}=155$ & 0.000363197 & 96534 \\
$m_{H}=160$ & 0.000183357 & 96523 \\
$m_{H}=165$ & 0.0000868488 & 95796 \\
$m_{H}=170$ & 0.0000827732 & 96799 \\
$m_{H}=175$ & 0.000101991 & 97212 \\
$m_{H}=180$ & 0.000171369 & 97628 \\
$m_{H}=185$ & 0.000385552 & 101749 \\
$m_{H}=190$ & 0.000485848 & 96785 \\
$m_{H}=195$ & 0.000504902 & 101078 \\
$m_{H}=200$ & 0.000493572 & 97456 \\
\hline
\end{tabular}


Table 3.3: RunIIb Pythia Higgs $g g \rightarrow H$ MC samples used in this analysis.

\begin{tabular}{lll}
\hline Higgs mass $(\mathrm{GeV})$ & $\sigma \times \mathrm{BF}(\mathrm{pb})$ & Events \\
\hline$m_{H}=115$ & 0.000108226 & 740817 \\
$m_{H}=120$ & 0.000174926 & 754773 \\
$m_{H}=125$ & 0.000258425 & 753569 \\
$m_{H}=130$ & 0.000345479 & 753029 \\
$m_{H}=135$ & 0.000421789 & 742588 \\
$m_{H}=140$ & 0.000473140 & 741999 \\
$m_{H}=145$ & 0.000487436 & 743460 \\
$m_{H}=150$ & 0.000455113 & 738003 \\
$m_{H}=155$ & 0.000363197 & 741793 \\
$m_{H}=160$ & 0.000183357 & 739552 \\
$m_{H}=165$ & 0.0000868488 & 748561 \\
$m_{H}=170$ & 0.0000827732 & 736956 \\
$m_{H}=175$ & 0.000101991 & 765029 \\
$m_{H}=180$ & 0.000171369 & 738659 \\
$m_{H}=185$ & 0.000385552 & 742679 \\
$m_{H}=190$ & 0.000485848 & 710983 \\
$m_{H}=195$ & 0.000504902 & 766659 \\
$m_{H}=200$ & 0.000493572 & 750555 \\
\hline
\end{tabular}


Table 3.4: RunIIa Pythia Higgs $Z H$ MC samples used in this analysis.

\begin{tabular}{|c|c|c|}
\hline \multicolumn{3}{|c|}{ RunIIa $Z H, H \rightarrow W W, Z Z, \gamma \gamma, e e, \mu \mu, \tau \tau, \gamma Z(W, Z$ incl } \\
\hline Mass $(\mathrm{GeV})$ & $\sigma \times B F(\mathrm{pb})$ & Events \\
\hline 115 & 0.0120 & 98633 \\
\hline 120 & 0.0110 & 100397 \\
\hline 125 & 0.0107 & 96955 \\
\hline 130 & 0.0108 & 96657 \\
\hline 135 & 0.0102 & 100133 \\
\hline 140 & 0.0098 & 100840 \\
\hline 145 & 0.0096 & 102044 \\
\hline 150 & 0.0091 & 100145 \\
\hline 155 & 0.0085 & 100987 \\
\hline 160 & 0.0077 & 100733 \\
\hline 165 & 0.0069 & 101373 \\
\hline 170 & 0.0061 & 100074 \\
\hline 175 & 0.0056 & 98952 \\
\hline 180 & 0.0051 & 101752 \\
\hline 185 & 0.0046 & 81001 \\
\hline 190 & 0.0042 & 100392 \\
\hline 195 & 0.0039 & 100317 \\
\hline 200 & 0.0035 & 101020 \\
\hline
\end{tabular}


Table 3.5: RunIIb Pythia Higgs $Z H$ MC samples used in this analysis.

\begin{tabular}{lcc}
\hline \multicolumn{2}{|c|}{ RunIlb1 $Z H, H \rightarrow W W, Z Z, \gamma \gamma, e e, \mu \mu, \tau \tau, \gamma Z(W, Z$ incl $)$} \\
\hline \hline Mass $(\mathrm{GeV})$ & $\sigma \times B F(\mathrm{pb})$ & Events \\
\hline 115 & 0.0120 & 2376073 \\
120 & 0.0110 & 2391298 \\
125 & 0.0107 & 2381811 \\
130 & 0.0108 & 2388843 \\
135 & 0.0102 & 2368533 \\
140 & 0.0098 & 2392601 \\
145 & 0.0096 & 2390534 \\
150 & 0.0091 & 2383950 \\
155 & 0.0084 & 2380440 \\
160 & 0.0077 & 2371777 \\
165 & 0.0069 & 2382746 \\
170 & 0.0061 & 2386161 \\
175 & 0.0056 & 2406100 \\
180 & 0.0051 & 2369629 \\
185 & 0.0046 & 2388035 \\
190 & 0.0042 & 2369554 \\
195 & 0.0039 & 2381589 \\
200 & 0.0035 & \\
\hline & & 237546 \\
\hline
\end{tabular}

As in the $g g \rightarrow H$ and $Z H$ cases, we also use PYTHIA MC for simulation of non-resonant $Z Z\left(Z Z \rightarrow \ell^{+} \ell^{-} \ell^{+} \ell^{-}\right)$and $t \bar{t}$ background events. In addition, we look for contributions from $Z Z \rightarrow \ell^{+} \ell^{-} \tau \tau$ where the taus decay into electrons or muons as appropriate to match our final signal signature. Contributions from $Z Z \rightarrow \tau \tau \tau \tau$ and their subsequent decays into muons and electrons were also examined, but found to be insignificant. 
The sample for the $Z Z \rightarrow$ eeee and $Z Z \rightarrow \mu \mu \mu \mu$ channels have a cross section times $\mathrm{BF}$ of $1.8 \mathrm{fb}$. The $Z Z \rightarrow e e \mu \mu$ channel has a cross section times $\mathrm{BF}$ of $3.6 \mathrm{fb}$. The $t \bar{t} \rightarrow 2 b+2 \ell+2 \nu$ sample used for background calculation consists of samples generated with top quark mass of $172 \mathrm{GeV}$ and a cross section times BF of $814 \mathrm{fb}$. More details on the MC samples above mentioned used for both RunIIa and RunIIb are given in Tables 3.6 through 3.7.

Table 3.6: RunIIa Pythia GEANT MC samples used in this analysis.

\begin{tabular}{|c|c|c|}
\hline \hline Physics Channel & $\sigma \times \mathrm{BF}(\mathrm{fb})$ & Events \\
\hline \hline$Z Z \rightarrow \mu \mu \mu \mu$ & 1.8 & 194,731 \\
\hline$Z Z \rightarrow e e e e$ & 1.8 & 193,160 \\
\hline$Z Z \rightarrow e e \mu \mu$ & 3.6 & 200,250 \\
\hline$Z Z \rightarrow \mu \mu \tau \tau$ & 3.6 & 208,500 \\
\hline$Z Z \rightarrow e e \tau \tau$ & 3.6 & 210,000 \\
\hline$t \bar{t} \rightarrow 2 b+2 \ell+2 \nu$ & 814 & $1,550,802$ \\
\hline \hline
\end{tabular}

Table 3.7: RunIIb Pythia GEANT MC samples used in this analysis.

\begin{tabular}{|c|c|c|}
\hline \hline Physics Channel & $\sigma \times \mathrm{BF}(\mathrm{fb})$ & Events \\
\hline \hline$Z Z \rightarrow \mu \mu \mu \mu$ & 1.8 & $1,581,265$ \\
\hline$Z Z \rightarrow e e e e$ & 1.8 & $1,576,593$ \\
\hline$Z Z \rightarrow e e \mu \mu$ & 3.6 & $2,511,549$ \\
\hline$Z Z \rightarrow 2 \mu 2 \tau$ & 3.6 & $1,116,879$ \\
\hline$Z Z \rightarrow 2 e 2 \tau$ & 3.6 & $1,112,168$ \\
\hline$t \bar{t} \rightarrow 2 b+2 \ell+2 \nu$ & 814 & $5,067,962$ \\
\hline \hline
\end{tabular}




\section{CHAPTER 4 OBJECT IDENTIFICATION}

We now describe the offline identification of objects used in this study: electrons and muons.

\subsection{Electrons}

In the $\mathrm{D} \varnothing$ event reconstruction an electromagnetic (EM) tower is defined by adding the energy measured by the calorimeter in all four EM layers plus the first hadronic (FH1) layer. EM clusters are formed from seed EM towers which have $E_{T}>500 \mathrm{MeV}$. Neighboring towers are added if they have $E_{T}>50 \mathrm{MeV}$ and if they are within $\Delta R=\sqrt{(\Delta \eta)^{2}+(\Delta \phi)^{2}}<$ 0.3 of the seed tower in the central region of the detector or within a cone radius of $10 \mathrm{~cm}$ in the third layer of the EM calorimeter in the end caps. These preclusters are used as starting points for final clusters if their energy exceeds $1 \mathrm{GeV}$.

In this analysis we employ the following selection criteria for all electrons used in the eeee and $е e \mu \mu$ channels to define 'good' electrons. We apply corrections to all electromagnetic clusters with $p_{T}>5 \mathrm{GeV}$. Details on the most recent set of corrections can be found in [28]. Further electron and photon identification (EMID) requirements use the so called "Point0" electron ID [29]. These selections are described below. 
- Particle Data Group (PDG) ID = 11 or 10 (Monte Carlo only)

- Energy fraction $\left(f_{E M}\right)$ - the energy of a cluster in the electromagnetic calorimeter $\left(E_{E M}\right)$ over the total energy $\left(E_{t o t}\right)$ in the hadronic and electromagnetic part of the calorimeter - must be $\geq 0.90$

- Detector $\eta\left(\eta_{\text {det }}\right)$ must be $\left|\eta_{\text {det }}\right|<1.1(\mathrm{CC})$ or $1.5<\left|\eta_{\text {det }}\right|<3.2$ (EC)

- Further CC specific selections:

Calorimeter isolation (Iso): electrons must be isolated in $\eta \times \phi$ space from surrounding clusters. A cone with radius $\Delta R<0.4$ is defined. Only $9 \%$ of the total energy within the cone $\Delta R<0.4$ is allowed to be outside the $\Delta R<0.2$ cone, or

$$
I s o=\frac{E_{t o t}(\Delta R<0.4)-E_{E M}(\Delta R<0.2)}{E_{E M}(\Delta R<0.2)}<0.09 .
$$

Track isolation of the EM cluster $\left(\right.$ IsoHC4): total track $p_{T}$ (for tracks with $p_{T}^{\text {track }}>0.5 \mathrm{GeV}$ ) in the hollow cone $0.05<R<0.4$ around the EM cluster. It must be $<4.0 \mathrm{GeV}$.

Probability of track matching $\left(\chi^{2}\right)>0.0$ OR Hit-on-Road Discriminant (HoR probability of finding a certain number of hits in the CFT and SMT tracks) $>0.6$. This method counts the number of fired CFT fibers or SMT pixels along a road (path of a charged particle). This number is then used to calculate the probability of a EM object to be an electron or a fake (photon).

Artificial neural network output $(\mathrm{ANN}-7)>0.4$. A neural network is trained with 7 input variables for the $\mathrm{CC}$ region in order to perform electron and photon discrimination [30].

- Further EC specific selections: 
$I s o \leq 0.10$

Lateral and longitudinal shapes of EM cluster $\leq 40$

$I$ soHC4 $<2.5 \mathrm{GeV} \times\left|\eta_{\text {det }}\right|+7.0 \mathrm{GeV}$

ANN-3 $>0.05$. A neural network is trained with 3 input variables for the EC region in order to perform electron and photon discrimination [30].

Shower width of the EM cluster in the $r-\phi$ plane $\left(\sigma_{\phi}\right)<100$

The data/MC scale factors are applied to MC electrons for the "Point0" EMID requirement and are parametrized as a function of luminosity, $\eta_{\text {det }}$ and $\phi_{\text {det }}$. These scale factors are applied to all the relevant distributions and numbers.

For the eeee analysis, we expand our electron acceptance into the intercryostat region (ICR) of the detector by allowing ICR eletrons, which are discarded in most analysis, to be included. These candidates are initially reconstructed as $\tau$ objects $^{1}$. The details of ICR electron reconstruction can be found in [31, 32, 33, 34], and are summarized below:

- Calorimeter $E_{T}>10 \mathrm{GeV}$

- $1.1<\eta_{\text {det }}<1.5$

- for type 1 tau objects, the intercryostat detector (ICD) energy fraction must be $\geq 0.1$

- The EM + ICD energy fraction must pass a minimum threshold that varies with $\eta_{\text {det }}$

- Must be track matched - track momentum is used to estimate ICR electron $p_{T}$

- For Run IIb, the following requirements are included:

\footnotetext{
${ }^{1}$ Type 1 tau : calorimeter cluster, 1 matched track, no associated EM subcluster, for $\tau \rightarrow \pi^{-} \nu_{\tau} ;$ type 2 tau: calorimeter cluster, 1 matched track, $\geq 1$ associated EM subclusters, for $\tau \rightarrow \rho^{-} \nu_{\tau} \rightarrow \pi^{0} \pi^{-} \nu_{\tau}$; type 3 tau: calorimeter cluster, $\geq 2$ matched tracks, mainly $\tau \rightarrow \pi^{-} \pi^{-} \pi^{+}\left(\pi^{0}\right) \nu_{\tau}$
} 
We search for type 1 and type 2 tau, but if the tau object was originally reconstructed as type 3, the best matched track is used in the tau Neural Network (NN) calculation

$$
\text { tau } \mathrm{NN}>0.2
$$

- For Run IIa, the following requirements are included:

$$
\text { tau } \mathrm{NN}>0.7
$$

If reconstructed as a type 3 tau, must have a type 3 likelihood $>0.15$

The data/MC scale factors are applied to ICR electrons in the MC and are parametrized as a function of $\eta_{\text {det }}$ and $\phi_{\text {det }}$. These scale factors are applied to all the relevant distributions and numbers.

\subsection{Muons}

The muon system uses segment information from the three muon layers (A, B and C) to provide unambiguous muon identification with modest momentum resolution. Segments from the B and C layers are combined into a single BC segment if they are consistent with a straight line. A muon that is identified only by its track segments in the muon system is called a "local muon". Track information from the central tracking system can also be used to help identify muons by matching a local muon to a central track. Additional information about minimal interacting particles (MIP) in the calorimeter is used in some cases as well. 
Two parameters are used to characterize muons [27]: type and quality. The type of a muon is represented by the name nseg and assumes the values $0, \pm 1, \pm 2$ and $\pm 3^{2}$. Positive values, including 0 , of nseg indicate that the local muon matches to a track in the central track system while negative values indicate there is not a match. After finding all nseg $>1$ muon tracks, we loop over all central tracks searching for a match between them and hits in the muon system that eventually were not found.

The muon quality can be "loose", "medium" or "mediumnseg3". Thus, based on both type and quality muons are classified as:

- $\mathbf{n s e g}=\mathbf{0}$ loose/medium: muons with $n s e g=0$ are muons with a hit matched with a central track or a central track matched to a muon track in the calorimeter.

- $\mathbf{n s e g}=+\mathbf{1}$ loose/medium: muons with nseg=1 are muons with an A segment matched with a central track. An nseg=1 is loose if it has at least one scintillator hit and at least two A layer wire hits. An nseg=1 is medium if it fullfills the above requirements and is located in the bottom part of the detector.

- $\mathbf{n s e g}=+\mathbf{2}$ loose/medium: $\mathrm{nseg}=2$ muons are muons with a $\mathrm{BC}$ segment matched to a central track. loose ones require at least one BC layer scintillator hit and at least two $\mathrm{BC}$ layer wire hits. An nseg=2 is medium if it fullfills the above requirements and is located in the bottom part of the detector.

- $\mathbf{n s e g}=+3$ medium/loose: muons with nseg=3 is medium (mediumnseg3) if it has at least two A layer wire hits, at least one A layer scintillator hit, at least two $\mathrm{BC}$ layer wire hits and at least one BC scintillator hit (only required in the forward region). An nseg=3 is defined as loose if one if the above requirements fails.

\footnotetext{
${ }^{2}=1$ has A layer segment only; = 2 has B,C or BC segment; $=3$ a segment both inside (A) or outside (B,C or $\mathrm{BC})$ the toroid
} 
These muon qualities also require the scintillator time be within 10 ns of beam-produced muons to veto on muons coming from cosmic rays (cosmic muons). If the time requirement is not present the qualities are named "looseNCV", "mediumNCV" and "mediumnseg3NCV".

We employ the following selection criteria for all muons used in the $\mu \mu \mu \mu$ and $е e \mu \mu$ channels to define 'good' muons [20, 27]:

- $|\eta|<2.5$

- looseNCV or nseg=0 quality

- Matched to a central track

- Muon tracks with SMT hits are required to have the distance of closest approach to the primary vertex (dca) in the transverse plane $<0.04 \mathrm{~cm}$; those without SMT hits are required to have dca $<0.2 \mathrm{~cm}$.

- Muon Isolation:

nseg $>0$ muons are isolated if $\frac{p_{T}(\text { track })}{p_{T}^{\mu}}<0.25$ and $\frac{E_{T}(\text { calhalo })-0.005 \mathcal{L}}{p_{T}^{\mu}}<0.4$. $p_{T}($ track $)$ is the scalar sum of all the $p_{T}$ of the tracks inside $\Delta R<0.4$ excluding the muon itself and $E_{T}$ (calhalo) is the sum of all the calorimeter $E_{T}$ in the annulus between $\Delta R=0.1$ and $\Delta R=0.4$, while $\mathcal{L}$ represents the instantaneous luminosity in units of $10^{30} \mathrm{~cm}^{-2} \mathrm{sec}^{-1}$. nseg $=0$ muons are isolated if $\frac{p_{T}(\text { track })}{p_{T}^{\mu}}<0.1$ and $\frac{E_{T}(\text { calhalo })-0.005 \mathcal{L}}{p_{T}^{\mu}}<0.1$.

The isolation requirements are applied in order to suppress QCD and $t \bar{t}$ background contamination where muons may be produced by quark decays into jets and therefore are expected to be accompanied by significant activity around the subject muon. These selections are similar to the "TrkLooseScaled" and "TopScaledTight" points defined in Ref. 
[27] except for the addition of the instantaneous luminosity term $-0.005 \mathcal{L}$. Adding this term increases the isolation efficiency as seen in Figs. 15 and 16 of D0 note 6025, giving an efficiency $>99 \%$ for nseg $>0$ muons and $>95 \%$ for nseg $=0$ muons. As the efficiency is so high, all muons in both the $\mu \mu \mu \mu$ and $е e \mu \mu$ analyses are required to be isolated.

The data/MC scale factors applied to the MC muons for the looseNCV requirement are parameterized as a function of instantaneous luminosity $(\mathcal{L}), \phi$, and $\eta_{\text {det }}[27]$. The data/MC scale factors applied to the MC muons for the central track requirement are parametrized as a function of the position of the primary vertex on the $z$ axis $\left(z_{v t x}\right)$ versus the value of $\eta$ in the CFT $\left(\eta_{c f t}\right)$ and $\left|\eta_{c f t}\right|$ vs $\mathcal{L}$. Isolation is parameterized in $\eta_{c f t}$ versus $p_{T}$ versus $\Delta R\left(\mu\right.$,closest jet) and $\left|\eta_{c f t}\right|$ versus $\mathcal{L}$. The isolation corrections are parameterized as a function of $\left|\eta_{c f t}\right|, p_{T}$ and $\Delta R$ between the muon and the closest $15 \mathrm{GeV}$ jet. These scale factors are applied to all the relevant distributions and numbers. From D0 Notes 6326 and 6025 , the RunIIb efficiencies for looseNCV vary from $90.62 \%$ to $91.4 \%$. Of this, about $82 \%$ are $n s e g=3,8 \%$ are $n s e g=2$, and $2 \%$ are $n s e g=1$ for muons from Z's, while nseg $=0$ adds about an additional 5\% per muon, and about an additional $24 \%$ for the 4 -muon acceptance (from 0.69 to 0.85 ). The track dca requirement efficiency varies from $90.7 \%$ to 92.4\%. The TopScaledTight efficiency varies from $93.3 \%$ to $95.1 \%$ while the efficiency for TrkLooseScaled varies from $98.3 \%$ to $99.0 \%$. Including the instantaneous luminosity term increases the isolation efficiencies to estimated values of $99.5 \pm 0.3 \%$ for $n s e g>0$ muons and $97.0 \pm 1.0 \%$ for nseg $=0$ muons. As that increase is not included in the MC scale factors, an ad hoc correction of $4 \%$ is made below to the $4 \mu$ acceptance.

We require the muon to be matched to a track as to get the best possible momentum measurement. The dca requirements helps to reduce possible junk tracks. About $90 \%$ of muon tracks have SMT hits; those who do not have their momentum corrected for the $\mathrm{z}$ of the vertex. Ref. [27] contains studies of muon momentum resolution and the amount 
of "oversmearing" that is needed for the MC to produce the same resolution as seen in the data. The difference in resolution between different tracking categories was seen to be small, and a momentum resolution of $0.02 \oplus 0.0025 p_{T}$ was determined. From this, it is about a $6 \sigma(4 \sigma)$ effect for a $p_{T}=60 \mathrm{Gev}(100 \mathrm{GeV})$ for a muon to have its charge flipped. As those events will also have their muon momentum badly measured, we will require that dimuon pairs have opposite charge. The acceptance loss is less than $0.3 \%$ and modelled by smeared MC.

\subsection{Missing Energy}

The presence of neutrinos in an event is inferred from an imbalance of net momentum in the plane perpendicular to the beam (transverse plane). This quantity is calculated from the vector sum of transverse momenta of all calorimeter cells that pass the jet-ID algorithm [36], except those in the coarse hadronic layers, which suffer from higher levels of noise. Coarse hadronic cells are only included if they are clustered within a reconstructed jet. This raw $\mathbb{E}_{T}$ is corrected for the energies of other objects, such as photons, electrons, $\tau$ 's, and jets in the event. As muons deposit only a small portion of their energy in the calorimeter, their momenta is subtracted from the $\mathbb{E}_{T}$ vector.

The missing transverse energy $\left(\mathscr{E}_{T}\right)$ is not needed for the cross section measurement, but is used in the Higgs boson search outlined in Chapter 7. For the $4 \mu$ and $2 e 2 \mu$ final states $E_{T}$ is used with all muon, electron, and jet corrections applied . Because we do not impose muon data quality on the $4 e$ final state, we do not apply muon corrections to the $\mathscr{E}_{T}$ in $4 e$ final state events. 


\section{CHAPTER 5 EVENT SELECTION}

This work consists of an extension with the full final D0 data sample of the cross section measurement $\sigma\left(p \bar{p} \rightarrow Z / \gamma^{*} Z / \gamma^{*}\right)$ [37] which was published in Phys. Rev. D. Therefore we apply the same selection criteria, i.e. we require all isolated leptons in the event to have $p_{T}>15 \mathrm{GeV}$ and each lepton pair invariant mass to be $>30 \mathrm{GeV}$. In $H \rightarrow Z Z^{*}$ one of the $Z^{\prime} s$ is produced "off-shell", and such cuts might not be the most suitable to improve signal efficiency. For instance, the lepton pair coming from the $Z^{*}$ decay has lower $p_{T}$ than the pairs coming from $Z$ decay due to a lower $Z^{*}$ mass. Thus looser cuts such as $p_{T}>10$ or $p_{T}>7 \mathrm{GeV}$ could have been applied. The lepton pair invariant mass cutoff itself would have a higher acceptance with a cut lower than the $>30 \mathrm{GeV}$ applied. However, in order to benefit from the work already done in the cross section measurement we decided to use the same selection criteria, which is described for each four lepton final state case in the following sections.

\subsection{Signal eeee channel}

The selections utilized for acquiring eeee events are given below:

- At least four "good" electrons 
- $p_{T}^{1}>15.0 \mathrm{GeV}, p_{T}^{2}>15.0 \mathrm{GeV}, p_{T}^{3}>15.0 \mathrm{GeV}$, and $p_{T}^{4}>15.0 \mathrm{GeV}$

- The number of CC electrons $N_{C C E M} \geq 2$

- The two best dielectron pairs are defined as the ones with the invariant masses closest to the $Z$ mass. We require both to have invariant masses $>30 \mathrm{GeV}$.

We break the eeee channel into four sub-channels which depend on the number of electrons in the CC and ICR regions: $N_{C C E M}=2$ and $N_{I C R E M}=0, N_{C C E M}=3$ and $N_{\text {ICREM }}=0, N_{C C E M} \geq 4$ and $N_{\text {ICREM }}=0$, and $N_{I C R E M}=1$. The splitting is applied because QCD background contamination is expected to vary significantly depending on the number of central electrons, which are required to be either track matched or have a significant number of hits in the tracking chamber, and because the jet background in the ICR region is greater than in the CC or EC. We only use ICR electrons within $1.1<$ $|\eta|<1.5$. As $\eta_{\text {det }}$ is calculated using different methods for tau objects and electromagnetic objects (electrons and photons), there are occasionally overlaps and we remove any ICR electron that is found to be within $\Delta R<0.5$ of a CC/EC electron to avoid double counting. ICR electrons are also required to have a track match. While the CC and EC electrons are not explicitly required to have a track match, we require those that are track matched to have $\Delta z_{D C A}\left(e_{C C / E C}, e_{I C R}\right)<3.0 \mathrm{~cm}$ to reduce QCD contamination. The cut flow for $Z Z \rightarrow$ eeee data in both RunIIa and RunIIb is shown in Tables 5.1 and 5.2.

where 1 ICR electron requires, necessarily, 2 or more CC electrons.

MC samples are used to measure the product of the geometrical acceptance and the event selection efficiency, Acc $\times \epsilon$, for each topology and for the various physics processes. Recall that, Signal represents either $H \rightarrow Z Z \rightarrow$ eeee or $Z H \rightarrow$ eeee processes; $Z Z \rightarrow$ eeee the non-resonant $Z Z$ background and Migration represents low mass $Z Z$ production where final lepton combinations and reconstruction errors can cause these events to appear 
as signal. The $2 e 2 \tau$ sample represents the case where pair produced $Z^{\prime}$ s decay into an elecetron and a $\tau$ pair, and then the $\tau$ 's either leptonically decay into electrons or else decay hadronically and then fake an electron. Plots of some kinematic variables associated with the eeee channel are shown in Fig. 5.1 through 5.2. In these plots, we refer to a best set of dielectron pairings. As we have four electrons and do not look at the electron charge, there are three possible sets of electron pairings in each events, e.g. $(12,34) ;(13,24)$; and $(14,23)$. We examine each pairing, excluding any pairing where either $Z$ has a mass less than $30 \mathrm{GeV}$. Of the remaining pairings, we select as the best pairing that which has one of the two dielectron masses closest to the $Z$ pole mass of $91.2 \mathrm{GeV}$. 
Table 5.1: RunIIa: Cut flow for eeee data for four subchannels $(=2,=3, \geq 4$ central electrons with no ICR electrons, and with 1 ICR electron).

\begin{tabular}{|c|c|c|c|c|}
\hline Cut & \multicolumn{4}{|c|}{ Number of Events } \\
\hline Initial & \multicolumn{4}{|c|}{36294680} \\
\hline$\geq 3$ good elec. & \multicolumn{4}{|c|}{4303} \\
\hline Data Quality & \multicolumn{4}{|c|}{3911} \\
\hline$\geq 4$ good elec. & \multicolumn{4}{|c|}{19} \\
\hline$p_{T}$ and ICR overlap removal & \multicolumn{4}{|c|}{1} \\
\hline$\geq 2$ CC elec. & \multicolumn{4}{|c|}{0} \\
\hline CC/ICR topology & $\begin{array}{c}2 \text { CC electrons } \\
0 \text { ICR electron } \\
0\end{array}$ & $\begin{array}{c}3 \text { CC electrons } \\
0 \text { ICR electron } \\
0\end{array}$ & $\begin{array}{c}4 \text { CC electrons } \\
0 \text { ICR electron } \\
0\end{array}$ & $\begin{array}{c}\text { 1 ICR electron } \\
0\end{array}$ \\
\hline mass & 0 & 0 & 0 & 0 \\
\hline
\end{tabular}

Table 5.2: RunIIb: Cut flow for eeee data for four subchannels $(=2,=3$ or $\geq 4$ central electrons with no ICR electrons, and with 1 ICR electron).

\begin{tabular}{|c|c|c|c|c|}
\hline 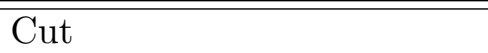 & \multicolumn{4}{|c|}{ Number of Events } \\
\hline Initial & \multicolumn{4}{|c|}{185982072} \\
\hline$\geq 3$ good elec. & \multicolumn{4}{|c|}{27238} \\
\hline Data Quality & \multicolumn{4}{|c|}{26059} \\
\hline$\geq 4$ good elec. & \multicolumn{4}{|c|}{117} \\
\hline$p_{T}$ and ICR overlap removal & \multicolumn{4}{|c|}{7} \\
\hline$\geq 2$ CC elec. & \multicolumn{4}{|c|}{5} \\
\hline & 2 CC electrons & $3 \mathrm{CC}$ electrons & 4 CC electrons & 1 ICR electron \\
\hline CC/ICR topoloov & 0 ICR electron & 0 ICR electron & 0 ICR electron & $?$ \\
\hline mass & 0 & $\frac{1}{1}$ & $\frac{2}{2}$ & $\frac{2}{2}$ \\
\hline
\end{tabular}


(a)

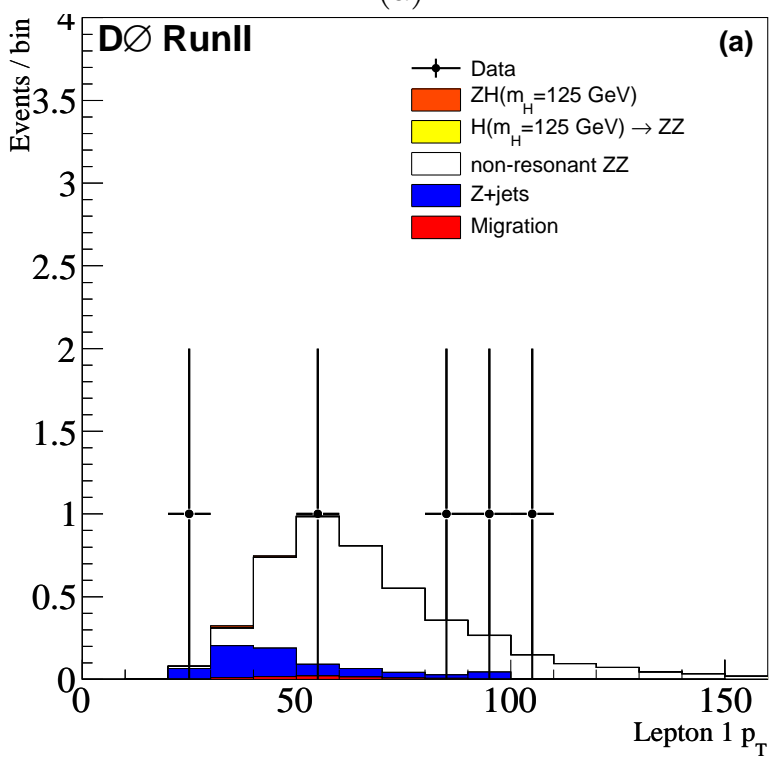

(c)

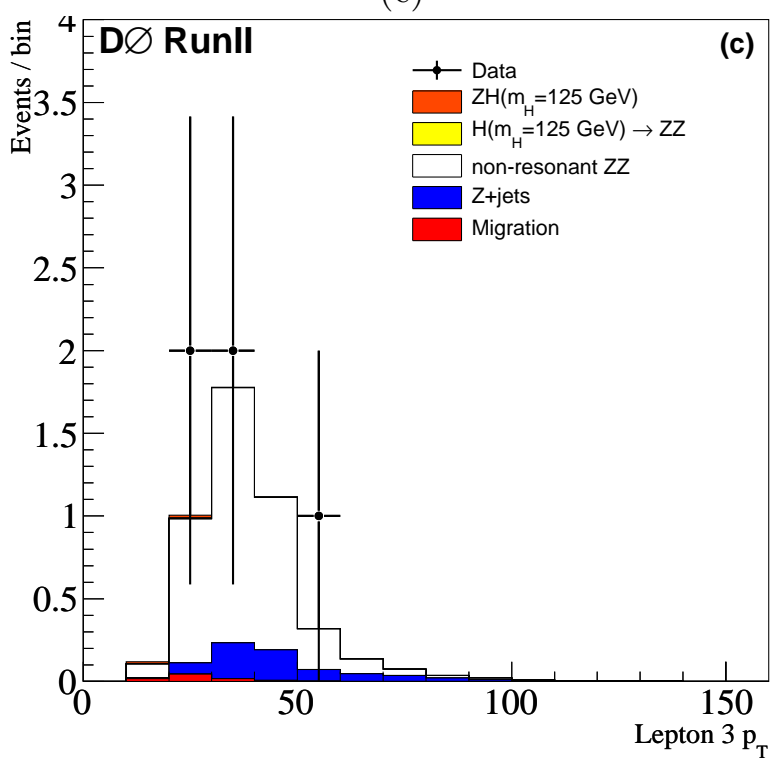

(b)

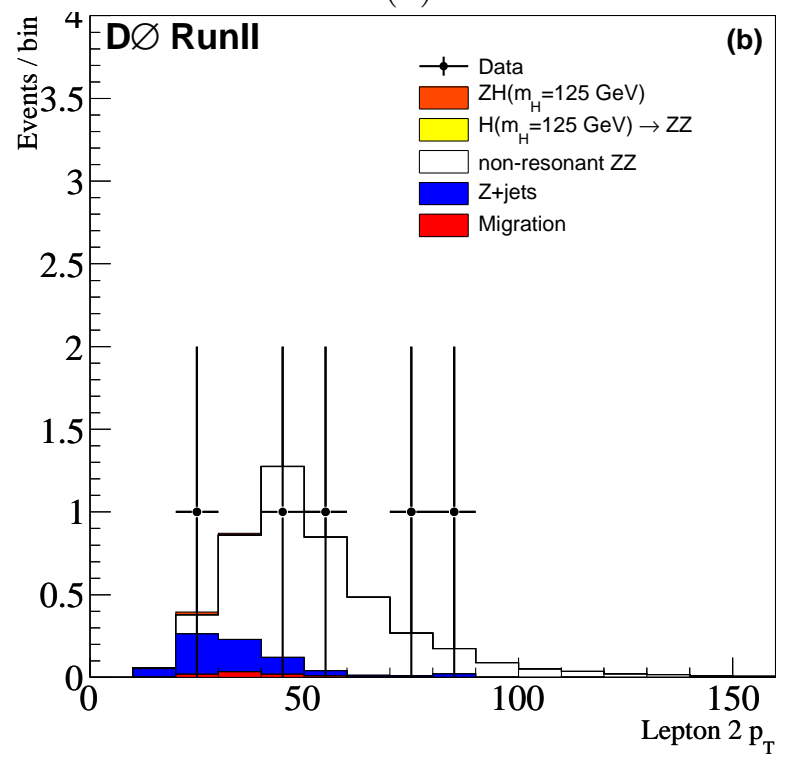

(d)

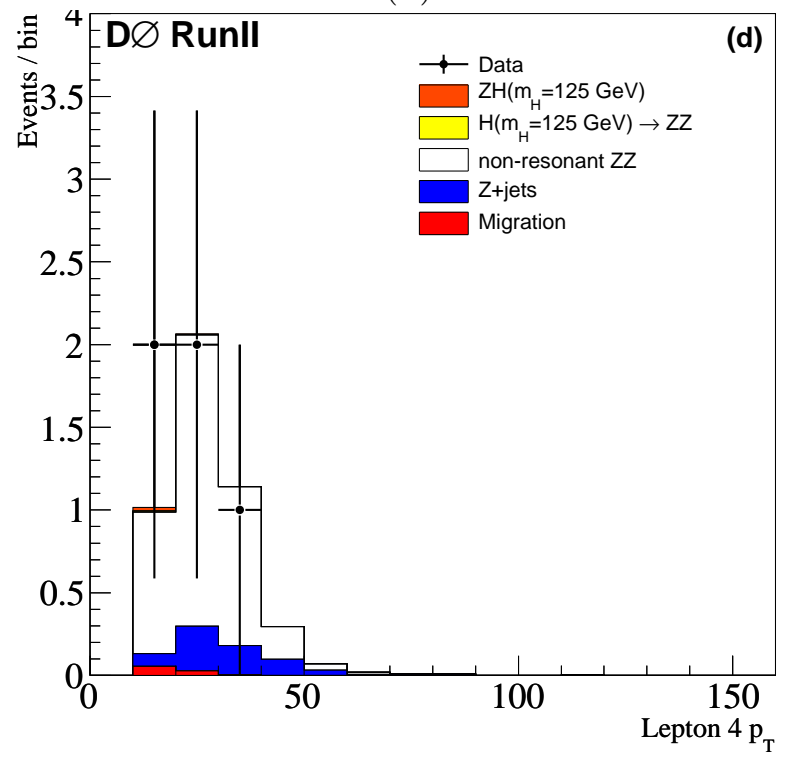

Figure 5.1: The $p_{T}$ distributions of the (a) lead, (b) second, (c) third, and (d) trailing electrons in four electron events, with data, expected Higgs signal (125 and $180 \mathrm{GeV})$ and backgrounds superimposed. 

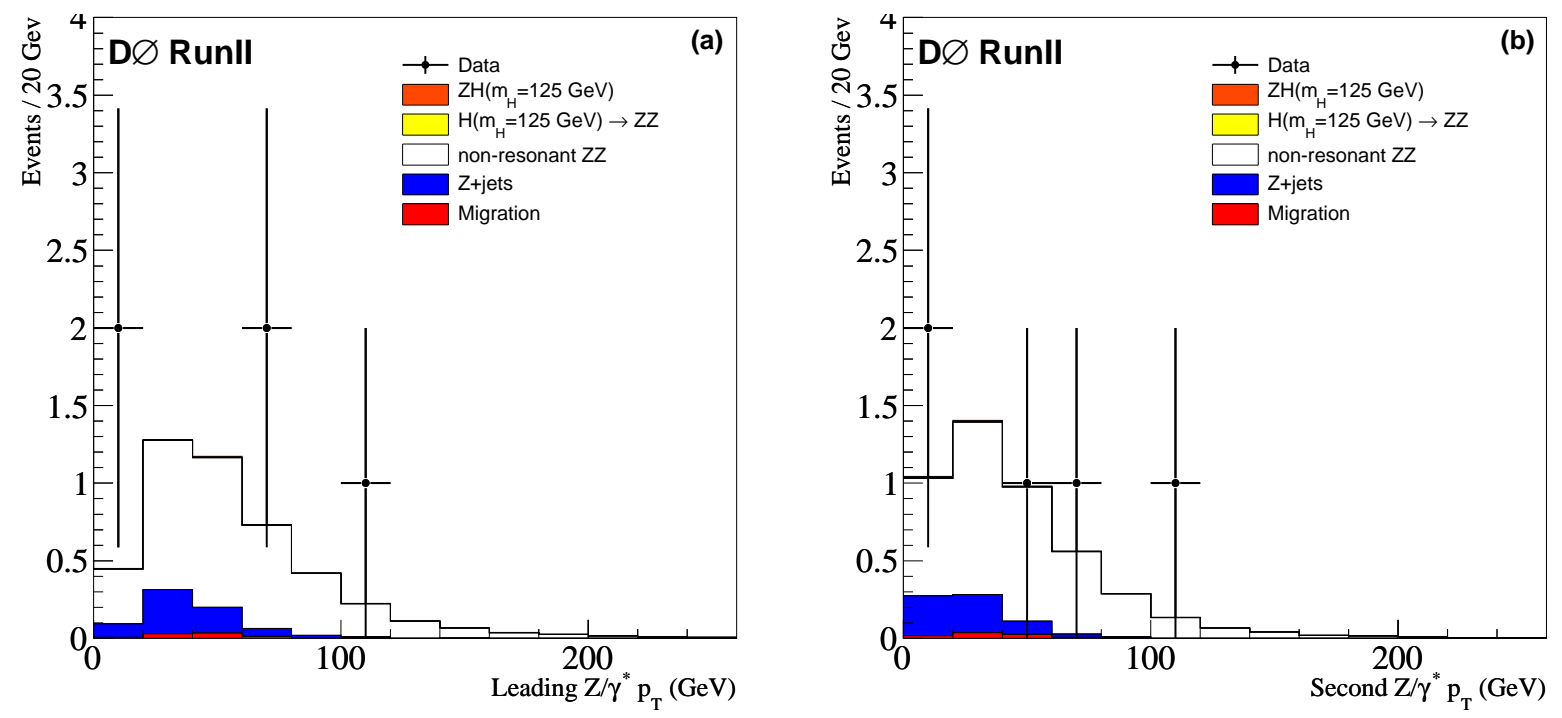

Figure 5.2: The $p_{T}$ of the lead and trailing ee pair in four electron events, where of the three potential sets of di-electron invariant masses in each event, we show only the one that has one of the pair masses closest to the $Z$ mass.
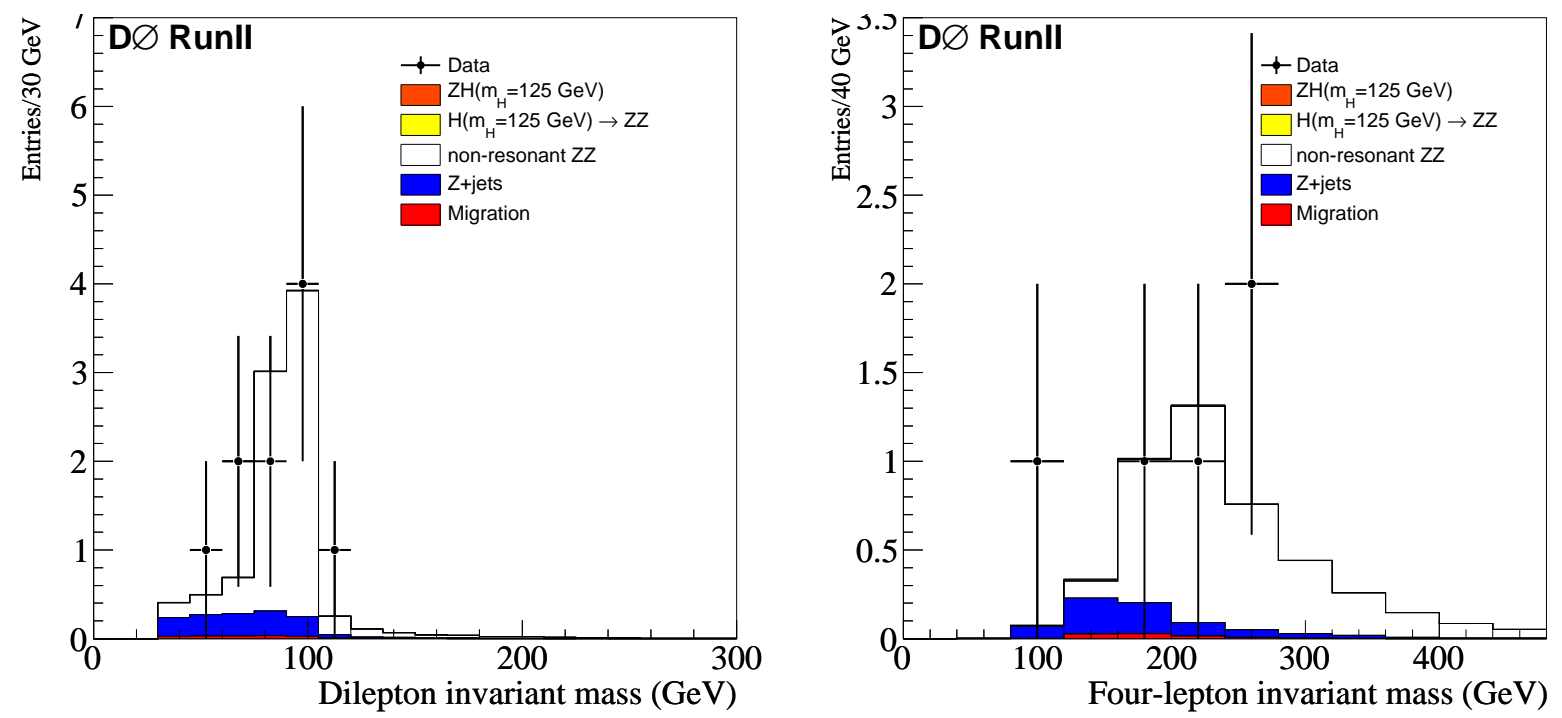

Figure 5.3: The di-electron (right) and four electron (left) mass distribution. As there are three potential sets of di-electron invariant masses in each event, we show only the one that has one of the pair masses closest to the $Z$ mass. 


\subsection{Signal $\mu \mu \mu \mu$ channel}

The selection utilized for $\mu \mu \mu \mu$ events are given below:

- At least 4 "good" muons as defined earlier

- $\Delta z_{D C A}<3.0 \mathrm{~cm}$ between all muon pair tracks

- All muons are required to be isolated

$$
\begin{aligned}
& - \text { nseg > } 0 \text { muons are "loose" isolated } \\
& - \text { nseg }=0 \text { muons are "tight" isolated }
\end{aligned}
$$

- Muon charges should have opposite sign (that is $\mu^{+} \mu^{-} \mu^{+} \mu^{-}$)

- All muon $p_{T}>15.0 \mathrm{GeV}$

- The invariant mass of the best dimuon pair $>30 \mathrm{GeV}$

- The invariant mass of the other dimuon pair $>30 \mathrm{GeV}$

The "best" dimuon pair is the set of oppositely charged muons whose invariant mass is closest to the $\mathrm{Z}$ mass. The cut flow for $\mu \mu \mu \mu$ data for both RunIIa and RunIIb is shown in Table 5.3. As a reference, weighted MC cut flows for $H \rightarrow Z Z \rightarrow \mu \mu \mu \mu$ and $Z H \rightarrow \mu \mu \mu \mu$ for two different mass points $\left(m_{H}=125\right.$ and $\left.m_{H}=180 \mathrm{GeV}\right)$ are shown in Tables 5.4 through 5.7. The weights for luminosity, $Z p_{T}$, and vertex $\mathrm{z}$ are applied at the initial stage. The corrections for the difference between data and MC muon efficiencies are applied after the four good muon selection, and labeled as "MuonCorr weighting" in this table. For $\mathrm{IIb}$, this correction is 0.948 for four muons, or 0.987 per muon. Plots of some kinematic variables associated with the $4 \mu$ channel are shown in Fig. 5.4 through 5.6. 
Table 5.3: RunII Cut flow for $\mu \mu \mu \mu$ Data

\begin{tabular}{l|c|c}
\hline \hline Cut & RunIIa Events & RunIIb Events \\
\hline Initial & 9776282 & 139915578 \\
\hline Data Quality & 9069996 & 133654953 \\
\hline Trigger & 9069845 & 133654953 \\
\hline 4 good muons & 1943 & 10476 \\
\hline Track $\Delta Z<3 \mathrm{~cm}$ & 815 & 4044 \\
\hline Isolation & 3 & 14 \\
\hline Muon charges & 3 & 10 \\
\hline Muon $p_{T}>15 \mathrm{GeV}$ & 0 & 3 \\
\hline Invariant mass $>30 \mathrm{GeV}$ & 0 & 3 \\
\hline \hline
\end{tabular}

Table 5.4: RunII Cut flow for $H \rightarrow Z Z \rightarrow \mu \mu \mu \mu$ weighted MC signal $\left(m_{H}=125 \mathrm{GeV}\right)$

\begin{tabular}{l|c|c}
\hline \hline Cut & RunIIa Events & RunIIb Events \\
\hline Initial & 96686 & 753569 \\
\hline Data Quality & 93022 & 683791 \\
\hline Loose muon & 93022 & 683791 \\
\hline Z Mass generated $>30 \mathrm{Gev}$ & 93022 & 683791 \\
\hline 4 good muons & 3504 & 18840 \\
\hline MuonCorr weighted & 2995 & 17701 \\
\hline Track $\Delta Z<3 \mathrm{~cm}$ & 2988 & 17057 \\
\hline Isolation & 2988 & 17057 \\
\hline$p_{T}>15 \mathrm{GeV}$ & 488 & 2573 \\
\hline Charge & 477 & 2505 \\
\hline Invariant mass reconstructed $>30 \mathrm{GeV}$ & 355 & 1862 \\
\hline \hline
\end{tabular}

The Acc $\times \epsilon$ cut flow values for the two Higgs mass points are given in Tables 5.8 through 5.11. The values for the non-resonant $Z Z$ channel are normalized to the mass $\left(M_{1}\left(Z / \gamma^{*}\right)>30 \mathrm{GeV}, M_{2}\left(Z / \gamma^{*}\right)>30\right)$ region. 
(a)

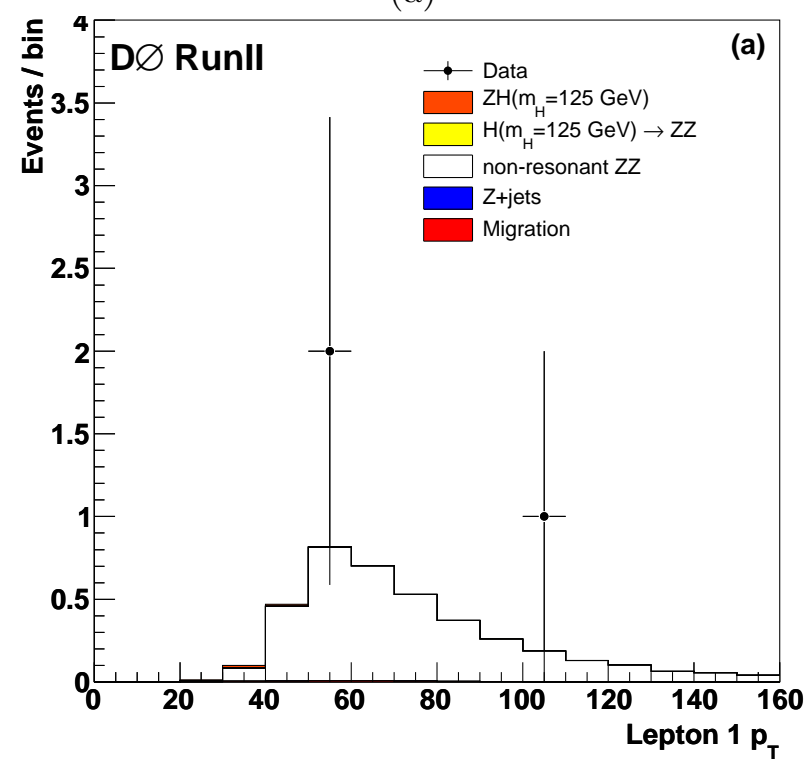

(c)

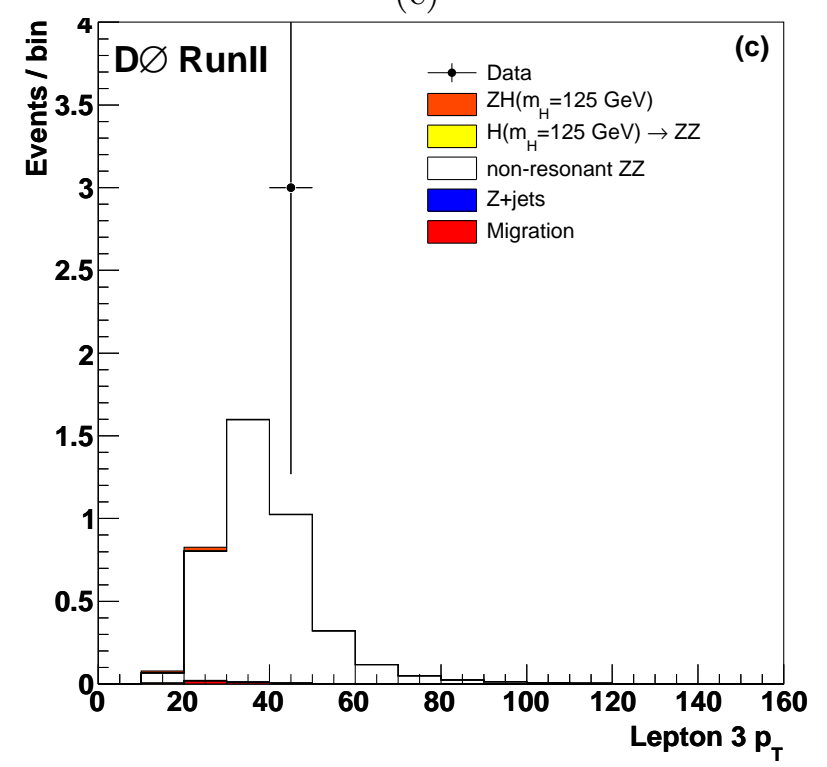

(b)

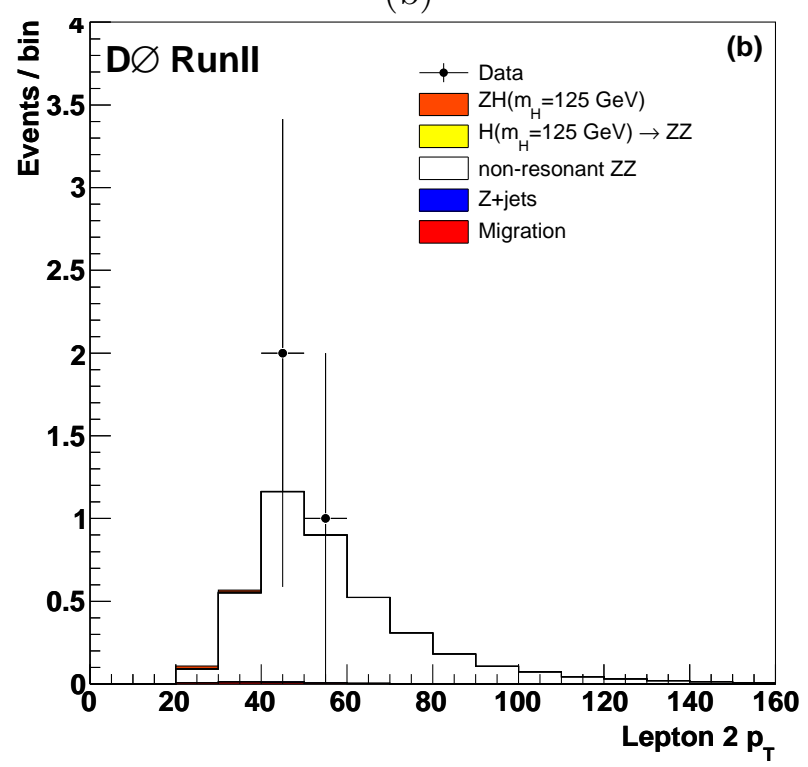

(d)

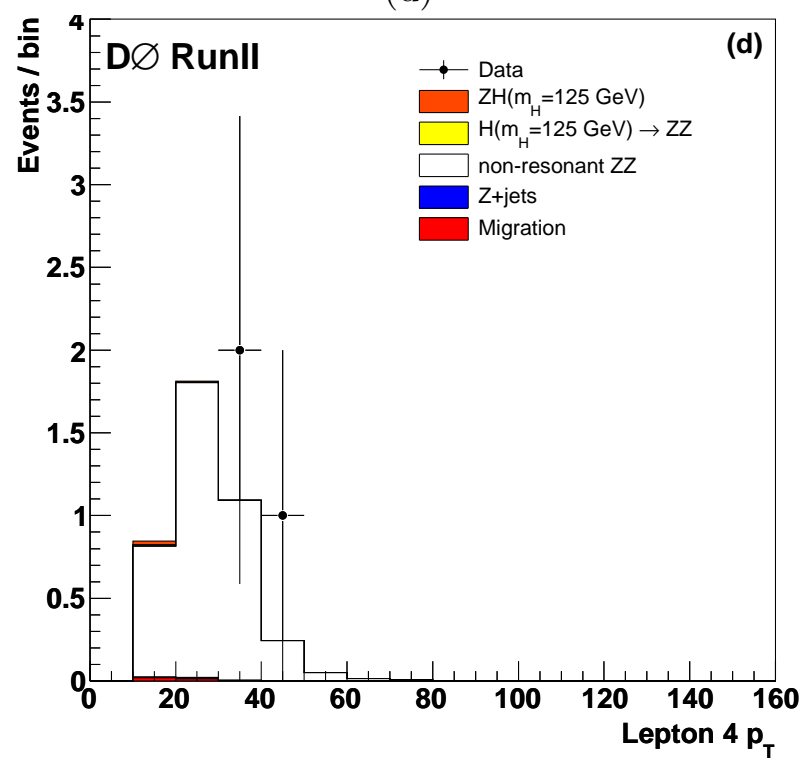

Figure 5.4: The $p_{T}$ distributions of the four muons ordered in $p_{T}$ in the $4 \mu$ channel, with data, expected Higgs signal (125 and $180 \mathrm{GeV}$ ) and backgrounds superimposed. 
Table 5.5: RunII Cut flow for $Z H \rightarrow \mu \mu \mu \mu$ weighted MC signal $\left(m_{H}=125 \mathrm{GeV}\right)$

\begin{tabular}{l|c|c}
\hline \hline Cut & RunIIa Events & RunIIb Events \\
\hline Initial & 488768 & 3377215 \\
\hline Data Quality & 471198 & 2999205 \\
\hline Loose muon & 471198 & 2999205 \\
\hline Z Mass generated $>30 \mathrm{Gev}$ & 471198 & 2999205 \\
\hline 4 good muons & 443 & 2290 \\
\hline MuonCorr weighted & 379 & 2076 \\
\hline Track $\Delta Z<3 \mathrm{~cm}$ & 374 & 1933 \\
\hline Isolation & 374 & 1933 \\
\hline$p_{T}>15 \mathrm{GeV}$ & 201 & 996 \\
\hline Charge & 193 & 965 \\
\hline Invariant mass reconstructed $>30 \mathrm{GeV}$ & 171 & 850 \\
\hline \hline
\end{tabular}

Table 5.6: RunII Cut flow for $H \rightarrow Z Z \rightarrow \mu \mu \mu \mu$ weighted MC signal $\left(m_{H}=180 \mathrm{GeV}\right)$

\begin{tabular}{l|c|c}
\hline \hline Cut & RunIIa Events & RunIIb Events \\
\hline Initial & 97628 & 738659 \\
\hline Data Quality & 94115 & 670099 \\
\hline Loose muon & 94115 & 670099 \\
\hline Z Mass generated $>30 \mathrm{Gev}$ & 94115 & 670099 \\
\hline 4 good muons & 4698 & 25030 \\
\hline MuonCorr weighted & 3997 & 23722 \\
\hline Track $\Delta Z<3 \mathrm{~cm}$ & 3992 & 22898 \\
\hline Isolation & 3992 & 22898 \\
\hline$p_{T}>15 \mathrm{GeV}$ & 3316 & 19166 \\
\hline Charge & 3237 & 18667 \\
\hline Invariant mass reconstructed $>30 \mathrm{GeV}$ & 3177 & 18257 \\
\hline \hline
\end{tabular}


Table 5.7: RunII Cut flow for $Z H \rightarrow \mu \mu \mu \mu$ weighted MC signal $\left(m_{H}=180 \mathrm{GeV}\right)$

\begin{tabular}{l|c|c}
\hline \hline Cut & RunIIa Events & RunIIb Events \\
\hline Initial & 97628 & 738659 \\
\hline Data Quality & 94115 & 670099 \\
\hline Loose muon & 94115 & 670099 \\
\hline Z Mass generated $>30 \mathrm{Gev}$ & 94115 & 670099 \\
\hline 4 good muons & 4698 & 25030 \\
\hline MuonCorr weighted & 3997 & 23722 \\
\hline Track $\Delta Z<3 \mathrm{~cm}$ & 3992 & 22898 \\
\hline Isolation & 3992 & 22898 \\
\hline$p_{T}>15 \mathrm{GeV}$ & 3316 & 19166 \\
\hline Charge & 3237 & 18667 \\
\hline Invariant mass reconstructed $>30 \mathrm{GeV}$ & 3177 & 18257 \\
\hline \hline
\end{tabular}



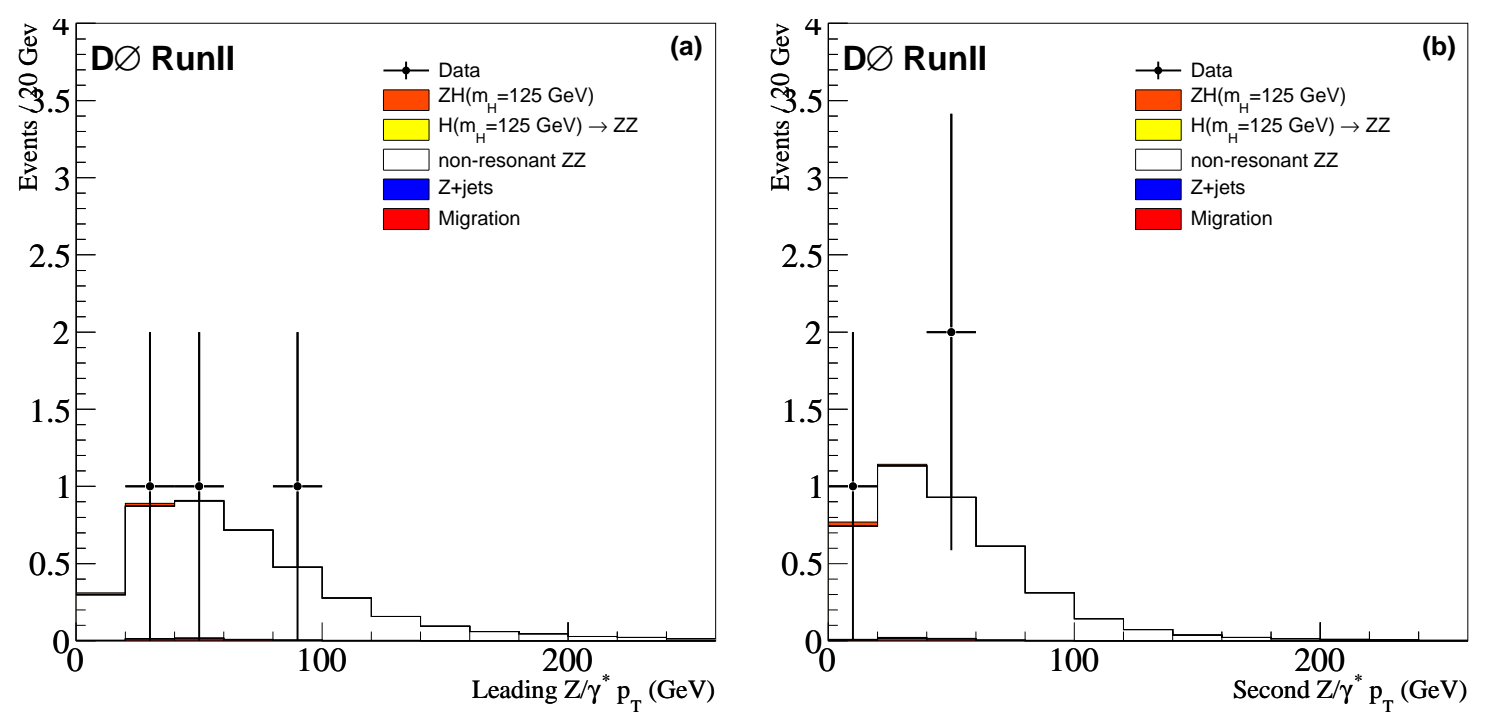

Figure 5.5: The $p_{T}$ distributions of leading $Z / \gamma^{*}$ and second $Z / \gamma^{*}$ in the $4 \mu$ channel, with data, expected Higgs signal (125 and $180 \mathrm{GeV}$ ) and backgrounds superimposed.

Table 5.8: Cut flow for RunII $H \rightarrow Z Z \rightarrow \mu \mu \mu \mu$ MC signal showing cumulative acceptance times efficiency $\left(m_{H}=125 \mathrm{GeV}\right)$

\begin{tabular}{l|c|c}
\hline \hline Cut & RunIIa & RunIIb \\
\hline MC ZZ Norm. & $1.0000 \pm 0.0000$ & $1.0000 \pm 0.0000$ \\
\hline 4 good muons & $0.0356 \pm 0.0006$ & $0.0260 \pm 0.0002$ \\
\hline Track $\Delta Z$ & $0.0304 \pm 0.0006$ & $0.0236 \pm 0.0002$ \\
\hline Isolation & $0.0304 \pm 0.0006$ & $0.0236 \pm 0.0002$ \\
\hline$p_{T}$ & $0.0050 \pm 0.0003$ & $0.0036 \pm 0.0001$ \\
\hline Charge & $0.0048 \pm 0.0022$ & $0.0035 \pm 0.0001$ \\
\hline Invariant mass & $0.0036 \pm 0.0002$ & $0.0026 \pm 0.0001$ \\
\hline \hline
\end{tabular}



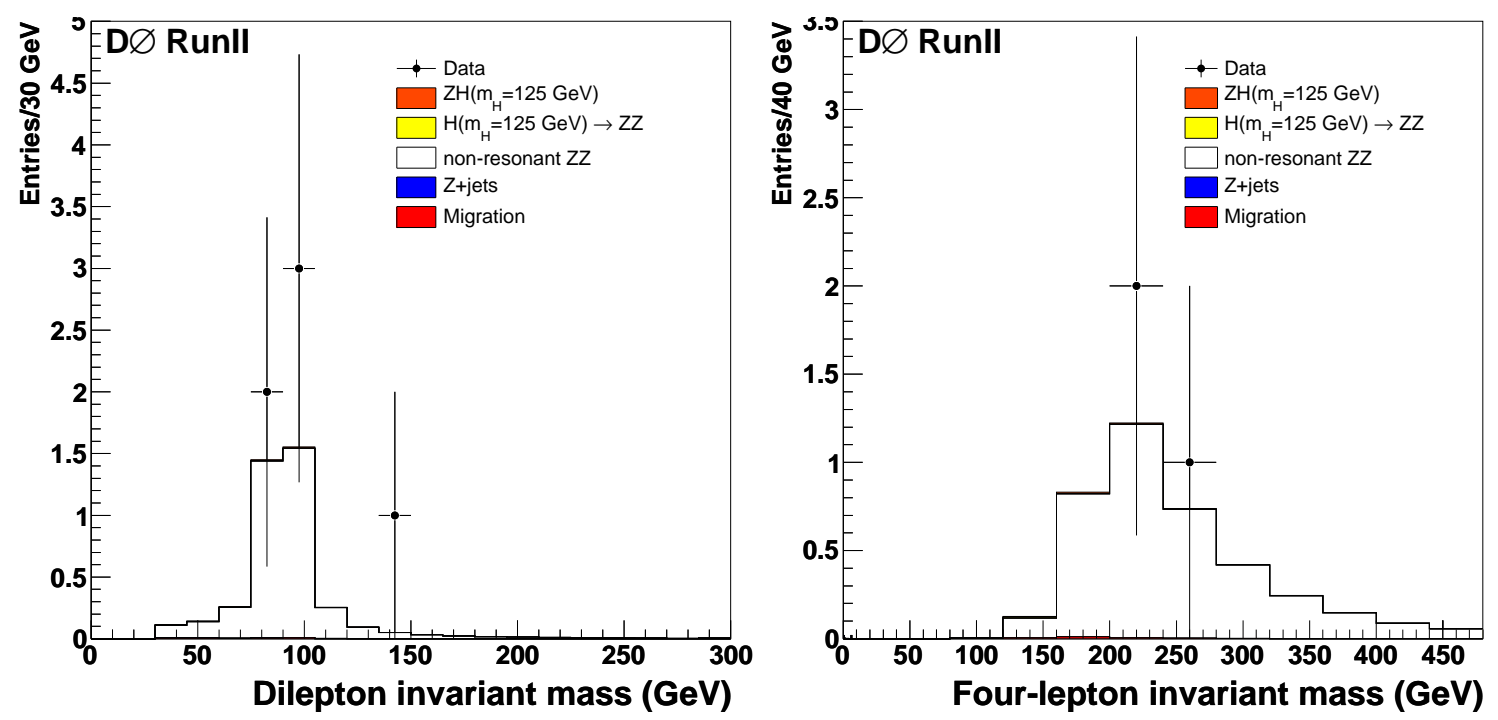

Figure 5.6: The distributions of the di-muon invariant mass of the best pair and four muon invariant mass in the $\mu \mu \mu \mu$ channel, with data, expected Higgs signal (125 and $180 \mathrm{GeV}$ ) and backgrounds superimposed.

Table 5.9: Cut flow for RunII $Z H \rightarrow \mu \mu \mu \mu$ MC signal showing cumulative acceptance times efficiency $\left(m_{H}=125 \mathrm{GeV}\right)$

\begin{tabular}{l|c|c}
\hline \hline Cut & RunIIa & RunIIb \\
\hline & Cumulative $A \times \epsilon$ & Cumulative $A \times \epsilon$ \\
\hline MC ZZ Norm. & $1.0000 \pm 0.0000$ & $1.0000 \pm 0.0000$ \\
\hline 4 good muons & $0.0010 \pm 0.0001$ & $0.0008 \pm 0.0001$ \\
\hline Track $\Delta Z$ & $0.0009 \pm 0.0001$ & $0.0007 \pm 0.0001$ \\
\hline Isolation & $0.0009 \pm 0.0001$ & $0.0007 \pm 0.0001$ \\
\hline$p_{T}$ & $0.0005 \pm 0.0001$ & $0.0004 \pm 0.0001$ \\
\hline Charge & $0.0004 \pm 0.0001$ & $0.0004 \pm 0.0001$ \\
\hline Invariant mass & $0.0004 \pm 0.0001$ & $0.0003 \pm 0.0001$ \\
\hline \hline
\end{tabular}

\subsubsection{Muon quality and charge}

The study requiring that muons coming from a $Z$ decay have opposite charges was done in order to improve the signal acceptance over background in the $\mu \mu \mu \mu$ channel. This 
Table 5.10: Cut flow for RunII $H \rightarrow Z Z \rightarrow \mu \mu \mu \mu$ MC signal showing cumulative acceptance times efficiency $\left(m_{H}=180 \mathrm{GeV}\right)$

\begin{tabular}{l|c|c}
\hline \hline Cut & RunIIa & RunIIb \\
\hline & Cumulative $A \times \epsilon$ & Cumulative $A \times \epsilon$ \\
\hline MC ZZ Norm. & $1.0000 \pm 0.0000$ & $1.0000 \pm 0.0000$ \\
\hline 4 good muons & $0.0461 \pm 0.0008$ & $0.0344 \pm 0.0003$ \\
\hline Track $\Delta Z$ & $0.0460 \pm 0.0008$ & $0.0315 \pm 0.0003$ \\
\hline Isolation & $0.0460 \pm 0.0008$ & $0.0315 \pm 0.0003$ \\
\hline$p_{T}$ & $0.0382 \pm 0.0008$ & $0.0263 \pm 0.0002$ \\
\hline Charge & $0.0317 \pm 0.0007$ & $0.0257 \pm 0.0002$ \\
\hline Invariant mass & $0.0312 \pm 0.0007$ & $0.0251 \pm 0.0002$ \\
\hline \hline
\end{tabular}

Table 5.11: Cut flow for RunII $Z H \rightarrow \mu \mu \mu \mu$ MC signal showing cumulative acceptance times efficiency $\left(m_{H}=180 \mathrm{GeV}\right)$

\begin{tabular}{l|c|c}
\hline \hline Cut & RunIIa & RunIIb \\
\hline & Cumulative $A \times \epsilon$ & Cumulative $A \times \epsilon$ \\
\hline MC ZZ Norm. & $1.0000 \pm 0.0000$ & $1.0000 \pm 0.0000$ \\
\hline 4 good muons & $0.0012 \pm 0.0008$ & $0.0011 \pm 0.0003$ \\
\hline Track $\Delta Z$ & $0.0011 \pm 0.0008$ & $0.0009 \pm 0.0003$ \\
\hline Isolation & $0.0011 \pm 0.0008$ & $0.0009 \pm 0.0003$ \\
\hline$p_{T}$ & $0.0008 \pm 0.0008$ & $0.0007 \pm 0.0002$ \\
\hline Charge & $0.0008 \pm 0.0007$ & $0.0007 \pm 0.0002$ \\
\hline Invariant mass & $0.0007 \pm 0.0007$ & $0.0006 \pm 0.0002$ \\
\hline \hline
\end{tabular}

cut takes the place of the previous acoplanarity cut as well as space angle cut to exclude backgrounds such as from $Z+$ jets events. All final muons are required to have opposite signs $2 \mu^{+} 2 \mu^{-}$. Tables $5.12-5.15$ show the results of such cut before the data quality cut was applied, which reduced the final number of events in the $\mu \mu \mu \mu$ channel from 7 to 3 . We observe that all the candidate muons are nseg $=2$ and $n s e g=3$, consistent with the relative acceptance of the various muon topologies (for 28 muons, one expects about 24-25 to be $\mathrm{nseg}=3,2-3$ to be $\mathrm{nseg}=2,0.5$ to be $n s e g=1$, and $0.9-1.5$ to be $n s e g=0)$. 
Table 5.12: Charge distribution for RunII $\mu \mu \mu \mu$ data

\begin{tabular}{c|c|c|c|c|c}
\hline \hline Type & Cut & Events & $2 \mu^{+} 2 \mu^{-}$ & $3 \mu^{+} \mu^{-}$or $3 \mu^{-} \mu^{+}$ & $\mu \mu \mu \mu^{+}$or $\mu \mu \mu \mu^{-}$ \\
\hline \multirow{3}{*}{} & 4 good muons & 1943 & 964 & 851 & 128 \\
\cline { 2 - 6 } & Track $\Delta Z<3 \mathrm{~cm}$ & 815 & 475 & 327 & 13 \\
\cline { 2 - 6 } & Isolation & 3 & 3 & 0 & 0 \\
\cline { 2 - 6 } & Charge and $p_{T}>15 \mathrm{GeV}$ & 0 & 0 & 0 & 0 \\
\cline { 2 - 6 } & Invariant mass $>30 \mathrm{GeV}$ & 0 & 0 & 0 & 642 \\
\hline \multirow{3}{*}{} & 4 good muons & 10476 & 5060 & 4774 & 02 \\
\cline { 2 - 6 } & Track $\Delta Z<3 \mathrm{~cm}$ & 4048 & 2343 & 1623 & 0 \\
\cline { 2 - 6 } & Isolation & 18 & 14 & 4 & 0 \\
\cline { 2 - 6 } & Charge and $p_{T}>15 \mathrm{GeV}$ & 7 & 7 & 0 & 0 \\
\cline { 2 - 6 } & Invariant mass $>30 \mathrm{GeV}$ & 7 & 7 & 0 & \\
\hline \hline
\end{tabular}

Table 5.13: Charge distribution for RunII $\mu \mu \mu \mu \mathrm{MC}$ signal

\begin{tabular}{c|c|c|c|c}
\hline \hline Type & Cut & $2 \mu^{+} 2 \mu^{-}$ & $3 \mu^{+} \mu^{-}$or $3 \mu^{-} \mu^{+}$ & $\mu \mu \mu \mu^{+}$or $\mu \mu \mu \mu^{-}$ \\
\hline \multirow{4}{*}{} & 4 good muons & 13945 & 126 & 1 \\
\cline { 2 - 5 } & Track $\Delta Z<3 \mathrm{~cm}$ & 13934 & 124 & 1 \\
\cline { 2 - 5 } & Isolation & 11995 & 83 & 0 \\
\cline { 2 - 5 } & charge and $p_{T}>15 \mathrm{GeV}$ & 10169 & 0 & 0 \\
\cline { 2 - 5 } & Invariant mass $>30 \mathrm{GeV}$ & 10037 & 0 & 11 \\
\hline \multirow{4}{*}{$\approx$} & 4 good muons & 59852 & 1378 & 3 \\
\cline { 2 - 5 } & Track $\Delta Z<3 \mathrm{~cm}$ & 58671 & 714 & 0 \\
\cline { 2 - 5 } & Isolation & 49897 & 425 & 0 \\
\cline { 2 - 5 } & Charge and $p_{T}>15 \mathrm{GeV}$ & 42239 & 0 & 0 \\
\cline { 2 - 5 } & Invariant mass $>30 \mathrm{GeV}$ & 41647 & 0 & \\
\hline \hline
\end{tabular}


Table 5.14: nseg distribution for RunII $\mu \mu \mu \mu$ data

\begin{tabular}{c|c|c|c|c|c|c}
\hline \hline Type & Cut & Events & nseg0 & nseg1 & nseg2 & nseg3 \\
\hline \multirow{4}{*}{} & 4 good muons & 1943 & 1511 & 54 & 1912 & 4295 \\
\cline { 2 - 7 } & Track $\Delta Z<3 \mathrm{~cm}$ & 815 & 28 & 16 & 409 & 2807 \\
\cline { 2 - 7 } & Isolation & 3 & 2 & 0 & 0 & 10 \\
\cline { 2 - 7 } & Charge and $p_{T}>15 \mathrm{GeV}$ & 0 & 0 & 0 & 0 & 0 \\
\cline { 2 - 7 } & Invariant mass $>30 \mathrm{GeV}$ & 0 & 0 & 0 & 0 & 0 \\
\hline \multirow{3}{*}{$\underset{3}{n}$} & 4 good muons & 10476 & 9444 & 505 & 5671 & 26284 \\
\cline { 2 - 7 } & Track $\Delta Z<3 \mathrm{~cm}$ & 4048 & 249 & 68 & 1806 & 14069 \\
\cline { 2 - 7 } & Isolation & 18 & 10 & 1 & 9 & 52 \\
\cline { 2 - 7 } & Charge and $p_{T}>15 \mathrm{GeV}$ & 7 & 0 & 0 & 3 & 25 \\
\cline { 2 - 7 } & Invariant mass $>30 \mathrm{GeV}$ & 7 & 0 & 0 & 3 & 25 \\
\hline \hline
\end{tabular}

Table 5.15: The fraction (\%) of muon topology (nseg) distribution for RunII $\mu \mu \mu \mu \mathrm{MC}$ signal

\begin{tabular}{c|c|c|c|c|c}
\hline \hline Type & Cut & nseg0 & nseg1 & nseg2 & nseg3 \\
\hline \multirow{3}{*}{} & 4 good muons & 4.2 & 4.2 & 6.0 & 85.7 \\
\cline { 2 - 6 } & Track $\Delta Z<3 \mathrm{~cm}$ & 4.2 & 4.2 & 6.0 & 85.7 \\
\cline { 2 - 6 } & Isolation & 4.3 & 4.5 & 5.9 & 85.2 \\
\cline { 2 - 6 } & Charge and $p_{T}>15 \mathrm{GeV}$ & 4.3 & 4.5 & 6.0 & 85.2 \\
\cline { 2 - 6 } & Invariant mass $>30 \mathrm{GeV}$ & 4.3 & 4.5 & 6.0 & 85.2 \\
\hline \multirow{3}{*}{} & 4 good muons & 5.2 & 4.0 & 5.6 & 85.1 \\
\cline { 2 - 6 } & Track $\Delta Z<3 \mathrm{~cm}$ & 4.9 & 4.0 & 5.6 & 85.5 \\
\cline { 2 - 6 } & Isolation & 5.1 & 4.3 & 5.6 & 85.0 \\
\cline { 2 - 6 } & Charge and $p_{T}>15 \mathrm{GeV}$ & 5.2 & 4.3 & 5.6 & 84.9 \\
\cline { 2 - 6 } & Invariant mass $>30 \mathrm{GeV}$ & 5.2 & 4.3 & 5.7 & 84.9 \\
\hline \hline
\end{tabular}




\subsection{Signal $е е \mu \mu$ channel}

The selections utilized for acquiring $e e \mu \mu$ events are given below:

- At least two "good" electrons

- At least two "good" muons

- $p_{T}^{1}>15.0 \mathrm{GeV}, p_{T}^{2}>15.0 \mathrm{GeV}$ for both electrons and muons

- Both muons are required to be isolated

- Cosine of space angle between muons is $\cos (\alpha)<0.96$. Thus, $\alpha>16.2^{\circ}(0.28$ radians $)$.

- Acoplanarity between any two muons must be greater than 0.05. (This is done to reject cosmic ray muons)

- $\Delta z_{D C A}<3.0 \mathrm{~cm}$ between all muon tracks

- $\Delta R>0.2$ between all electron-muon pairs

- Require one lepton pair invariant mass to be $M_{l l}>30 \mathrm{GeV}$ and the other to be $M_{l^{\prime} l^{\prime}}>30 \mathrm{GeV}$

We break the $е е \mu \mu$ channel into three sub-channels depending on the number of electrons in the CC region: $N_{C C E M}=0, N_{C C E M}=1$, and $N_{C C E M} \geq 2$. The splitting is applied because QCD background contamination is expected to vary significantly depending on the number of central electrons. The cut flow for $Z Z \rightarrow e e \mu \mu$ data both in RunIIa and RunIIb is shown in Tables 5.16 and 5.17.

The Acc $\times \epsilon$ cut flow values for $H \rightarrow Z Z \rightarrow e e \mu \mu$ and $Z H \rightarrow e e \mu \mu$ for two different mass points $\left(m_{h}=125\right.$ and $\left.m_{h}=180 \mathrm{GeV}\right) \mathrm{MC}$ signal and non-resonant $Z Z$ are shown in 
Table 5.16: RunIIa: Cut flow for $e e \mu \mu$ data for three subchannels $(=0,=1$ or $\geq 2$ central electrons).

\begin{tabular}{l|c|c|c}
\hline \hline Cut & \multicolumn{3}{|c}{ Number of Events } \\
\hline Initial & \multicolumn{3}{|c}{36229860} \\
\hline Data Quality & \multicolumn{3}{|c}{83513823} \\
\hline$\geq 1$ good elec. & \multicolumn{3}{|c}{32304} \\
\hline$\geq 1$ good muon & \multicolumn{3}{|c}{18} \\
\hline$p_{T}$ & 0 CC electrons & CC electrons & 2 CC electrons \\
\hline & 2 & 5 & 11 \\
\hline CC topology & 2 & 5 & 11 \\
\hline$\mu \cos \alpha$ & & 4 & 10 \\
and $\mu \mu$ Acoplanarity Cut & 2 & 1 & 0 \\
\hline$\mu$ track $\Delta z$ & 1 & 1 & 0 \\
\hline$\Delta R_{e \mu}$ & 1 & & \\
\hline mass & & & \\
\hline \hline
\end{tabular}

Table 5.17: RunIIb: Cut flow for $e e \mu \mu$ data for three subchannels $(=0,=1$ or $\geq 2$ central electrons).

\begin{tabular}{|c|c|c|c|}
\hline Cut & \multicolumn{3}{|c|}{ Number of Events } \\
\hline Initial & \multicolumn{3}{|c|}{185982072} \\
\hline Data Quality & \multicolumn{3}{|c|}{178582022} \\
\hline$\geq 1$ good elec. & \multicolumn{3}{|c|}{7235058} \\
\hline$\geq 1$ good muon & \multicolumn{3}{|c|}{2595} \\
\hline \multirow[t]{2}{*}{$p_{T}$} & \multicolumn{3}{|c|}{368} \\
\hline & 0 CC electrons & $1 \mathrm{CC}$ electrons & $2 \mathrm{CC}$ electrons \\
\hline CC topology & 23 & 97 & 248 \\
\hline $\begin{array}{l}\mu \cos \alpha \\
\text { and } \mu \mu \text { Acoplanarity Cut }\end{array}$ & 22 & 96 & 244 \\
\hline$\mu$ track $\Delta z$ & 3 & 25 & 168 \\
\hline$\Delta R_{e \mu}$ & 1 & 0 & 2 \\
\hline mass & 1 & 0 & 2 \\
\hline
\end{tabular}

Tables 5.18 through 5.25 for the ee $\mu \mu$ channel. These values are normalized to the mass $\left(M_{1}\left(Z / \gamma^{*}\right)>30 \mathrm{GeV}, M_{2}\left(Z / \gamma^{*}\right)>30 \mathrm{GeV}\right)$ region. 
Table 5.18: RunIIa: Cut flow for $H \rightarrow Z Z \rightarrow e e \mu \mu \mathrm{MC}$ signal $\left(m_{H}=125 \mathrm{GeV}\right)$ showing cumulative acceptance times efficiency values for three subchannels $(=0,=1$ or $\geq 2$ central electrons). Only statistical errors are shown.

\begin{tabular}{l|c|c|c}
\hline \hline Cut & \multicolumn{3}{|c}{ Cumulative $A \times \epsilon$} \\
\hline MC ZZ Norm. & \multicolumn{3}{|c}{$0.0000 \pm 0.0000$} \\
\hline $\begin{array}{l}\text { Z } 1 \text { good electron } \\
\text { and } \geq 1 \text { good muon }\end{array}$ & \multicolumn{3}{|c}{$0.0554 \pm 0.0010$} \\
\hline$\mu$ track $\Delta z$ & \multicolumn{3}{|c}{$0.0087 \pm 0.0004$} \\
\hline$p_{T}$ & $\begin{array}{c}\text { CC electrons } \\
\text { CC electrons }\end{array}$ & $\begin{array}{c}\text { CC electrons } \\
\text { Cumulative } A \times \epsilon\end{array}$ \\
\hline \multirow{2}{*}{ num CC elec. } & $0.00024 \pm 0.00006$ & $0.00182 \pm 0.00018$ & $0.00660 \pm 0.00034$ \\
\hline$\mu \cos \alpha$ and $\mu \mu$ & $0.00024 \pm 0.00006$ & $0.00182 \pm 0.00018$ & $0.00660 \pm 0.00034$ \\
acoplanarity cut & $0.00019 \pm 0.00006$ & $0.00138 \pm 0.00015$ & $0.00533 \pm 0.00030$ \\
\hline$\Delta R_{e \mu}$ & $0.00014 \pm 0.00005$ & $0.00095 \pm 0.00013$ & $0.00370 \pm 0.00025$ \\
\hline mass & \multicolumn{3}{|c}{} \\
\hline \hline
\end{tabular}

Table 5.19: RunIIa: Cut flow for $Z H \rightarrow e e \mu \mu$ MC signal $\left(m_{H}=125 \mathrm{GeV}\right)$ showing cumulative acceptance times efficiency values for three subchannels $(=0,=1$ or $\geq 2$ central electrons). Only statistical errors are shown.

\begin{tabular}{|c|c|c|c|}
\hline Cut & \multicolumn{3}{|c|}{ Cumulative $A \times \epsilon$} \\
\hline MC ZZ Norm. & \multicolumn{3}{|c|}{$1.0000 \pm 0.0000$} \\
\hline $\begin{array}{l}\geq 1 \text { good electron } \\
\text { and } \geq 1 \text { good muon }\end{array}$ & \multicolumn{3}{|c|}{$0.0031 \pm 0.0010$} \\
\hline$\mu \operatorname{track} \Delta z$ & \multicolumn{3}{|c|}{$0.0026 \pm 0.0010$} \\
\hline$p_{T}$ & \multicolumn{3}{|c|}{$0.0010 \pm 0.0004$} \\
\hline & $\begin{array}{c}0 \mathrm{CC} \text { electrons } \\
\text { Cumulative } A \times \epsilon\end{array}$ & $\begin{array}{c}1 \text { CC electrons } \\
\text { Cumulative } A \times \epsilon\end{array}$ & $\begin{array}{c}2 \mathrm{CC} \text { electrons } \\
\text { Cumulative } A \times \epsilon\end{array}$ \\
\hline num CC elec. & $0.00003 \pm 0.00006$ & $0.00022 \pm 0.00018$ & $0.00074 \pm 0.00034$ \\
\hline $\begin{array}{l}\mu \cos \alpha \text { and } \mu \mu \\
\text { acoplanarity cut }\end{array}$ & $0.00003 \pm 0.00006$ & $0.00022 \pm 0.00018$ & $0.00074 \pm 0.00034$ \\
\hline$\Delta R_{e \mu}$ & $0.00003 \pm 0.00006$ & $0.00018 \pm 0.00015$ & $0.00047 \pm 0.00030$ \\
\hline mass & $0.00002 \pm 0.00005$ & $0.00018 \pm 0.00013$ & $0.00039 \pm 0.00025$ \\
\hline
\end{tabular}


Table 5.20: RunIIa: Cut flow for $H \rightarrow Z Z \rightarrow e e \mu \mu \mathrm{MC}$ signal $\left(m_{H}=180 \mathrm{GeV}\right)$ showing cumulative acceptance times efficiency values for three subchannels $(=0,=1$ or $\geq 2$ central electrons). Only statistical errors are shown.

\begin{tabular}{l|c|c|c}
\hline \hline Cut & \multicolumn{3}{|c}{ Cumulative $A \times \epsilon$} \\
\hline MC ZZ Norm. & \multicolumn{3}{|c}{$0.0000 \pm 0.0000$} \\
\hline $\begin{array}{l}\text { Z good electron } \\
\text { and } \geq 1 \text { good muon }\end{array}$ & \multicolumn{3}{|c}{$0.0708 \pm 0.0010$} \\
\hline$\mu$ track $\Delta z$ & \multicolumn{3}{|c}{$0.0536 \pm 0.0009$} \\
\hline$p_{T}$ & $\begin{array}{c}\text { CC electrons } \\
\text { CC electrons }\end{array}$ & $\begin{array}{c}\text { CC electrons } \\
\text { Cumulative } A \times \epsilon\end{array}$ \\
\hline \multirow{2}{*}{ num CC elec. } & $0.00032 \pm 0.00007$ & $0.01414 \pm 0.00049$ & $0.03915 \pm 0.00080$ \\
\hline$\mu \cos \alpha$ and $\mu \mu$ & $0.00032 \pm 0.00007$ & $0.01412 \pm 0.00049$ & $0.03905 \pm 0.00080$ \\
acoplanarity cut & $0.00032 \pm 0.00007$ & $0.01354 \pm 0.00048$ & $0.03586 \pm 0.00077$ \\
\hline$\Delta R_{e \mu}$ & $0.00028 \pm 0.00007$ & $0.01342 \pm 0.00048$ & $0.03518 \pm 0.00076$ \\
\hline mass & \multicolumn{3}{|c}{} \\
\hline \hline
\end{tabular}

Table 5.21: RunIIa: Cut flow for $Z H \rightarrow e e \mu \mu \mathrm{MC}$ signal $\left(m_{H}=180 \mathrm{GeV}\right)$ showing cumulative acceptance times efficiency values for three subchannels $(=0,=1$ or $\geq 2$ central electrons). Only statistical errors are shown.

\begin{tabular}{l|c|c|c}
\hline \hline Cut & \multicolumn{3}{|c}{ Cumulative $A \times \epsilon$} \\
\hline MC ZZ Norm. & \multicolumn{3}{|c}{$0.0000 \pm 0.0000 \pm 0.0011$} \\
\hline $\begin{array}{l}\geq 1 \text { good electron } \\
\text { and } \geq 1 \text { good muon }\end{array}$ & \multicolumn{3}{|c}{$0.0036 \pm 0.0010$} \\
\hline$\mu$ track $\Delta z$ & \multicolumn{3}{|c}{$0020 \pm 0.0009$} \\
\hline$p_{T}$ & $\begin{array}{c}\text { 1 CC electrons } \\
\text { CC electrons }\end{array}$ & $\begin{array}{c}\text { CC electrons } \\
\text { Cumulative } A \times \epsilon\end{array}$ \\
\hline \multirow{2}{*}{$\begin{array}{c}\text { num CC elec. } \\
\mu \cos \alpha \text { and } \mu \mu\end{array}$} & $0.00002 \pm 0.00007$ & $0.00037 \pm 0.00049$ & $0.00159 \pm 0.00080$ \\
\hline acoplanarity cut & $0.00002 \pm 0.00007$ & $0.00037 \pm 0.00049$ & $0.00159 \pm 0.00080$ \\
\hline$\Delta R_{e \mu}$ & $0.00002 \pm 0.00007$ & $0.00028 \pm 0.00048$ & $0.00103 \pm 0.00077$ \\
\hline mass & $0.00002 \pm 0.00007$ & $0.00026 \pm 0.00048$ & $0.00090 \pm 0.00076$ \\
\hline \hline
\end{tabular}


Table 5.22: RunIIb: Cut flow for $H \rightarrow Z Z \rightarrow e e \mu \mu$ MC signal $\left(m_{H}=125 \mathrm{GeV}\right)$ showing cumulative acceptance times efficiency values for three subchannels $(=0,=1$ or $\geq 2$ central electrons). Only statistical errors are shown.

\begin{tabular}{|c|c|c|c|}
\hline Cut & \multicolumn{3}{|c|}{ Cumulative $A \times \epsilon$} \\
\hline MC ZZ Norm. & \multicolumn{3}{|c|}{$1.0000 \pm 0.0000$} \\
\hline $\begin{array}{l}\geq 1 \text { good electron } \\
\text { and } \geq 1 \text { good muon }\end{array}$ & \multicolumn{3}{|c|}{$0.0518 \pm 0.0004$} \\
\hline$\mu$ track $\Delta z$ & \multicolumn{3}{|c|}{$0.0449 \pm 0.0004$} \\
\hline$p_{T}$ & \multicolumn{3}{|c|}{$0.0075 \pm 0.0002$} \\
\hline \multirow[b]{2}{*}{ num CC elec. } & $\begin{array}{c}0 \text { CC electrons } \\
\text { Cumulative } A \times \epsilon\end{array}$ & $\begin{array}{c}1 \text { CC electrons } \\
\text { Cumulative } A \times \epsilon\end{array}$ & $\begin{array}{c}2 \text { CC electrons } \\
\text { Cumulative } A \times \epsilon\end{array}$ \\
\hline & $0.00016 \pm 0.00003$ & $0.00148 \pm 0.00008$ & $0.00750 \pm 0.00016$ \\
\hline $\begin{array}{l}\mu \cos \alpha \text { and } \mu \mu \\
\text { acoplanarity cut }\end{array}$ & $0.00016 \pm 0.00003$ & $0.00147 \pm 0.00008$ & $0.00585 \pm 0.00016$ \\
\hline$\Delta R_{e \mu}$ & $0.00015 \pm 0.00003$ & $0.00120 \pm 0.00008$ & $0.00435 \pm 0.00013$ \\
\hline mass & $0.00010 \pm 0.00001$ & $0.00087 \pm 0.00006$ & $0.00296 \pm 0.00011$ \\
\hline
\end{tabular}

Table 5.23: RunIIb: Cut flow for $Z H \rightarrow e e \mu \mu \mathrm{MC}$ signal $\left(m_{H}=125 \mathrm{GeV}\right)$ showing cumulative acceptance times efficiency values for three subchannels $(=0,=1$ or $\geq 2$ central electrons). Only statistical errors are shown.

\begin{tabular}{|c|c|c|c|}
\hline Cut & \multicolumn{3}{|c|}{ Cumulative $A \times \epsilon$} \\
\hline MC ZZ Norm. & \multicolumn{3}{|c|}{$1.0000 \pm 0.0000$} \\
\hline $\begin{array}{l}\geq 1 \text { good electron } \\
\text { and } \geq 1 \text { good muon }\end{array}$ & \multicolumn{3}{|c|}{$0.0032 \pm 0.0004$} \\
\hline$\mu$ track $\Delta z$ & \multicolumn{3}{|c|}{$0.0028 \pm 0.0004$} \\
\hline$p_{T}$ & \multicolumn{3}{|c|}{$0.0010 \pm 0.0002$} \\
\hline \multirow[b]{2}{*}{ num CC elec. } & $\begin{array}{c}0 \text { CC electrons } \\
\text { Cumulative } A \times \epsilon\end{array}$ & $\begin{array}{c}1 \text { CC electrons } \\
\text { Cumulative } A \times \epsilon\end{array}$ & $\begin{array}{c}2 \text { CC electrons } \\
\text { Cumulative } A \times \epsilon\end{array}$ \\
\hline & $0.00002 \pm 0.00003$ & $0.00023 \pm 0.00008$ & $0.00077 \pm 0.00016$ \\
\hline $\begin{array}{l}\mu \cos \alpha \text { and } \mu \mu \\
\text { acoplanarity cut }\end{array}$ & $0.00002 \pm 0.00003$ & $0.00023 \pm 0.00008$ & $0.00077 \pm 0.00016$ \\
\hline$\Delta R_{e \mu}$ & $0.00002 \pm 0.00003$ & $0.00016 \pm 0.00008$ & $0.00043 \pm 0.00013$ \\
\hline mass & $0.00002 \pm 0.00001$ & $0.00014 \pm 0.00006$ & $0.00036 \pm 0.00011$ \\
\hline
\end{tabular}


Table 5.24: RunIIb: Cut flow for $H \rightarrow Z Z \rightarrow e e \mu \mu$ MC signal $\left(m_{H}=180 \mathrm{GeV}\right)$ showing cumulative acceptance times efficiency values for three subchannels $(=0,=1$ or $\geq 2$ central electrons). Only statistical errors are shown.

\begin{tabular}{|c|c|c|c|}
\hline Cut & \multicolumn{3}{|c|}{ Cumulative $A \times \epsilon$} \\
\hline MC ZZ Norm. & \multicolumn{3}{|c|}{$1.0000 \pm 0.0000$} \\
\hline $\begin{array}{l}\geq 1 \text { good electron } \\
\text { and } \geq 1 \text { good muon }\end{array}$ & \multicolumn{3}{|c|}{$0.0688 \pm 0.0005$} \\
\hline$\mu$ track $\Delta z$ & \multicolumn{3}{|c|}{$0.0598 \pm 0.0005$} \\
\hline$p_{T}$ & \multicolumn{3}{|c|}{$0.0444 \pm 0.0004$} \\
\hline \multirow[b]{2}{*}{ num CC elec. } & $\begin{array}{c}0 \text { CC electrons } \\
\text { Cumulative } A \times \epsilon\end{array}$ & $\begin{array}{c}1 \text { CC electrons } \\
\text { Cumulative } A \times \epsilon\end{array}$ & $\begin{array}{c}2 \text { CC electrons } \\
\text { Cumulative } A \times \epsilon\end{array}$ \\
\hline & $0.00035 \pm 0.00004$ & $0.01630 \pm 0.00022$ & $0.04581 \pm 0.00037$ \\
\hline $\begin{array}{l}\mu \cos \alpha \text { and } \mu \mu \\
\text { acoplanarity cut }\end{array}$ & $0.00035 \pm 0.00004$ & $0.01629 \pm 0.00022$ & $0.04576 \pm 0.00037$ \\
\hline$\Delta R_{e \mu}$ & $0.00033 \pm 0.00004$ & $0.01511 \pm 0.00021$ & $0.03993 \pm 0.00034$ \\
\hline mass & $0.00031 \pm 0.00004$ & $0.01493 \pm 0.00021$ & $0.03911 \pm 0.00034$ \\
\hline
\end{tabular}

Table 5.25: RunIIb: Cut flow for $Z H \rightarrow e e \mu \mu \mathrm{MC}$ signal $\left(m_{H}=180 \mathrm{GeV}\right)$ showing cumulative acceptance times efficiency values for three subchannels $(=0,=1$ or $\geq 2$ central electrons). Only statistical errors are shown.

\begin{tabular}{|c|c|c|c|}
\hline Cut & \multicolumn{3}{|c|}{ Cumulative $A \times \epsilon$} \\
\hline MC ZZ Norm. & \multicolumn{3}{|c|}{$1.0000 \pm 0.0000$} \\
\hline $\begin{array}{l}\geq 1 \text { good electron } \\
\text { and } \geq 1 \text { good muon }\end{array}$ & \multicolumn{3}{|c|}{$0.0044 \pm 0.0005$} \\
\hline$\mu$ track $\Delta z$ & \multicolumn{3}{|c|}{$0.0037 \pm 0.0005$} \\
\hline$p_{T}$ & \multicolumn{3}{|c|}{$0.0018 \pm 0.0004$} \\
\hline \multirow[b]{2}{*}{ num CC elec. } & $\begin{array}{c}0 \text { CC electrons } \\
\text { Cumulative } A \times \epsilon\end{array}$ & $\begin{array}{c}1 \text { CC electrons } \\
\text { Cumulative } A \times \epsilon\end{array}$ & $\begin{array}{c}2 \text { CC electrons } \\
\text { Cumulative } A \times \epsilon\end{array}$ \\
\hline & $0.00002 \pm 0.00004$ & $0.00033 \pm 0.00022$ & $0.00144 \pm 0.00037$ \\
\hline $\begin{array}{l}\mu \cos \alpha \text { and } \mu \mu \\
\text { acoplanarity cut }\end{array}$ & $0.00002 \pm 0.00004$ & $0.00033 \pm 0.00022$ & $0.00144 \pm 0.00037$ \\
\hline$\Delta R_{e \mu}$ & $0.00002 \pm 0.00004$ & $0.00024 \pm 0.00021$ & $0.00081 \pm 0.00034$ \\
\hline mass & $0.00002 \pm 0.00004$ & $0.00022 \pm 0.00021$ & $0.00071 \pm 0.00034$ \\
\hline
\end{tabular}


The $p_{T}$ distributions for the four leptons in the $e e \mu \mu$ channel following all cuts are shown in Fig. 5.7 while Fig. 5.8 gives the dilepton invariant mass and 4-lepton mass distributions. 

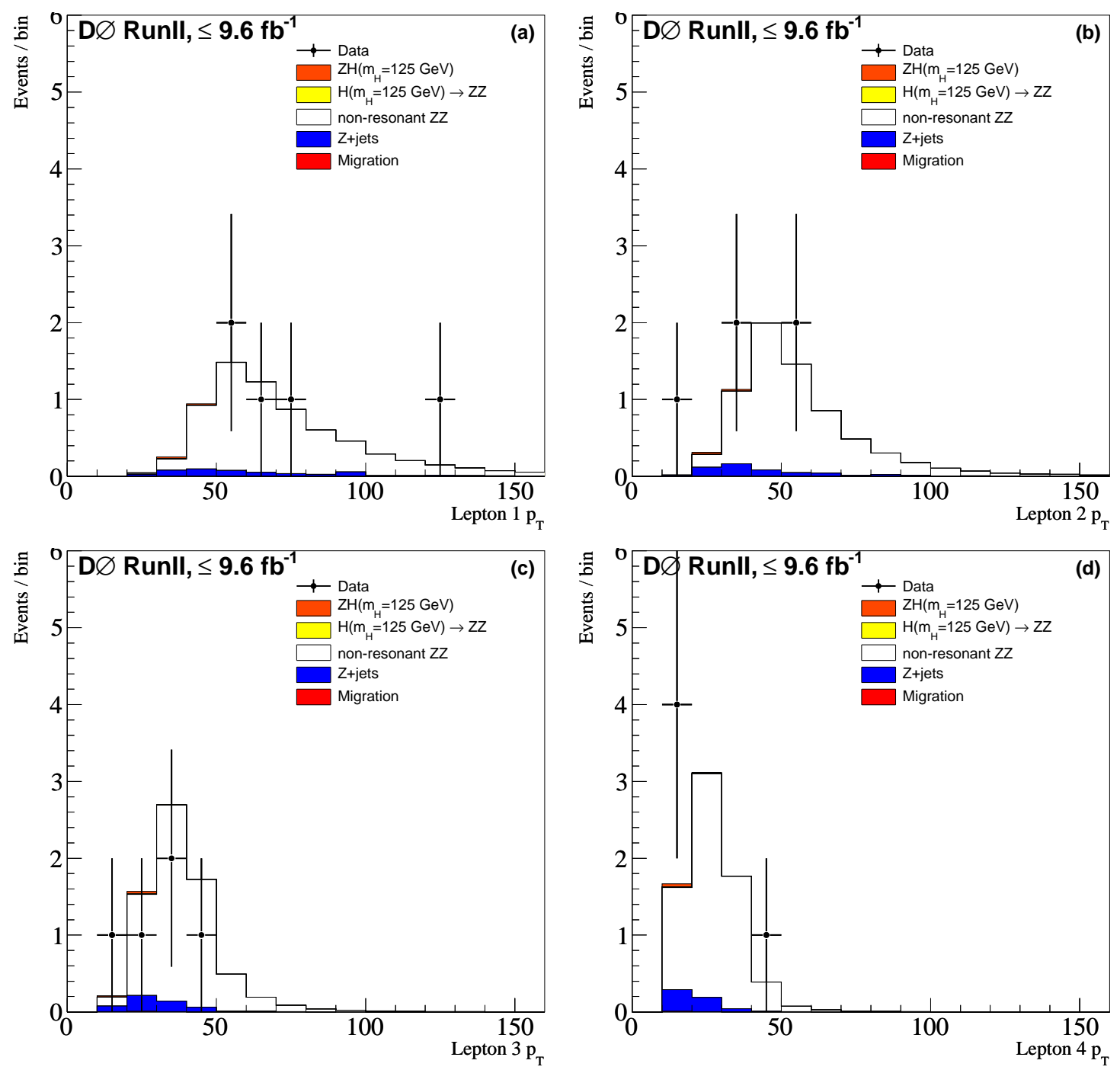

Figure 5.7: Distributions of the lepton $p_{T}$ ordered in $p_{T}$ for data, expected Higgs signal (125 and $180 \mathrm{GeV}$ ) and backgrounds superimposed for the ee $\mu \mu$ channel. 

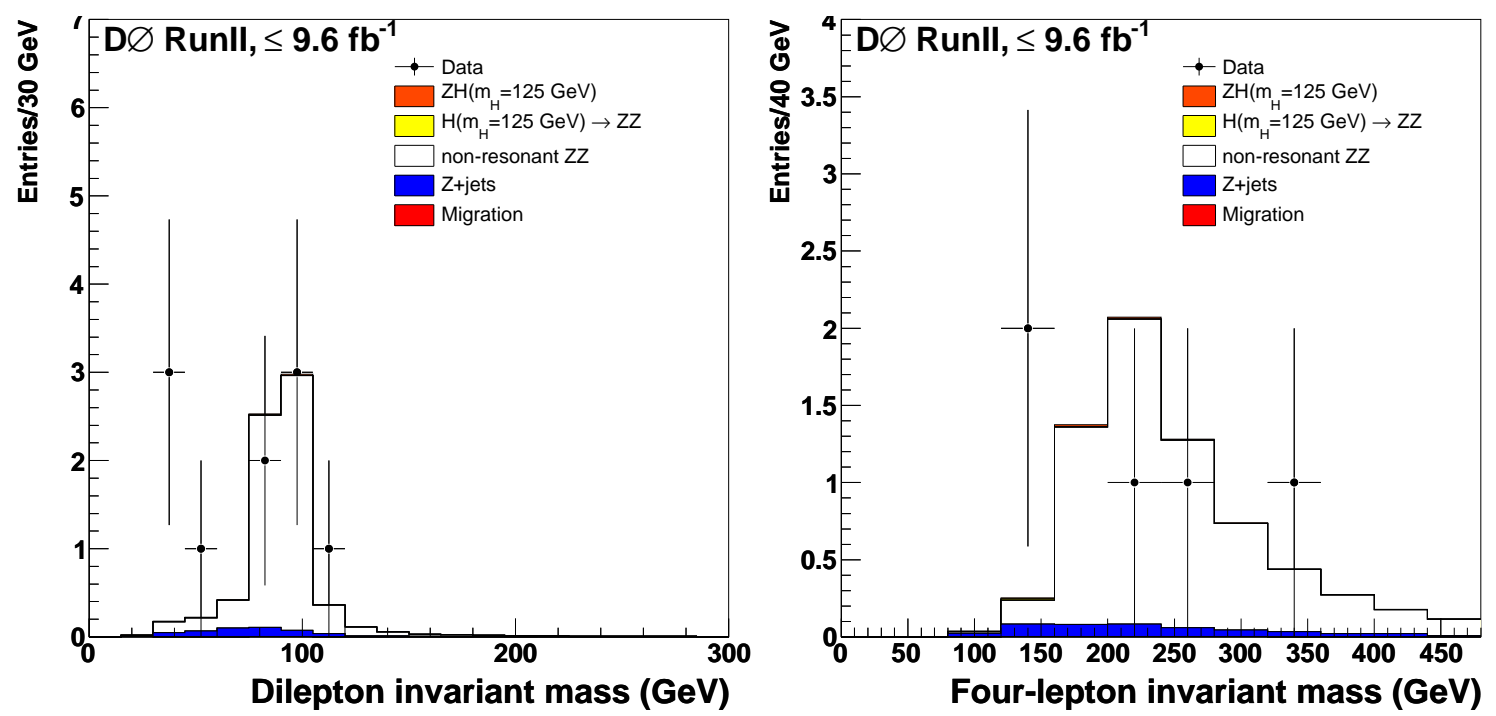

Figure 5.8: Distributions of dilepton invariant mass and four lepton invariant mass for data, expected Higgs signal (125 and $180 \mathrm{GeV}$ ) and backgrounds superimposed for the ee $\mu \mu$ channel. 


\section{4 non-resonant ZZ Background}

\subsection{1 eeee channel}

The $A c c \times \epsilon$ cut flow values for non-resonant $Z Z$ background are shown in Tables 5.26 and 5.27 for the eeee channel. The $Z Z$ channel is normalized to the mass $\left(M_{1}\left(Z / \gamma^{*}\right)>\right.$ $\left.30 \mathrm{GeV}, M_{2}\left(Z / \gamma^{*}\right)>30\right)$ region.

Table 5.26: RunIIa: Cut flow for non-resonant $Z Z \rightarrow$ eeee MC background showing cumulative acceptance times efficiency for three subchannels $(=2,=3$ or $\geq 4$ central electrons, = 1 ICR electron).

\begin{tabular}{|c|c|c|c|c|}
\hline Cut & \multicolumn{4}{|c|}{ Cumulative $A \times \epsilon$} \\
\hline $\begin{array}{l}\text { MC ZZ } \\
\text { Norm. }\end{array}$ & \multicolumn{4}{|c|}{$1.0000 \pm 0.0000$} \\
\hline $\begin{array}{l}\geq 4 \text { good } \\
\text { elec. }\end{array}$ & \multicolumn{4}{|c|}{$0.3349 \pm 0.0035$} \\
\hline $\begin{array}{l}p_{T} \text { and } \\
\text { overlap } \\
\text { removal }\end{array}$ & \multicolumn{4}{|c|}{$0.2813 \pm 0.0031$} \\
\hline & $\begin{array}{c}2 \mathrm{CC} / 0 \mathrm{ICR} \\
\text { Cumulative } A \times \epsilon\end{array}$ & $\begin{array}{c}3 \text { CC/0 ICR } \\
\text { Cumulative } A \times \epsilon\end{array}$ & $\begin{array}{c}4 \text { CC/0 ICR } \\
\text { Cumulative } A \times \epsilon\end{array}$ & $\begin{array}{c}\geq 2 / 1 \text { ICR } \\
\text { Cumulative } A \times \epsilon\end{array}$ \\
\hline $\begin{array}{l}\text { num } \\
\text { CC/ICR } \\
\text { electrons }\end{array}$ & $0.0309 \pm 0.0011$ & $0.0702 \pm 0.0016$ & $0.0658 \pm 0.0015$ & $0.0901 \pm 0.0018$ \\
\hline mass & $0.0309 \pm 0.0011$ & $0.0702 \pm 0.0016$ & $0.0658 \pm 0.0015$ & $0.0901 \pm 0.0018$ \\
\hline
\end{tabular}


Table 5.27: RunIIb: Cut flow for non-resonant $Z Z \rightarrow$ eeee $\mathrm{MC}$ background showing cumulative acceptance times efficiency for three subchannels $(=2,=3$ or $\geq 4$ central electrons, = 1 ICR electron).

\begin{tabular}{l|c|c|c|c}
\hline \hline Cut & \multicolumn{4}{|c}{ Cumulative $A \times \epsilon$} \\
\hline $\begin{array}{l}\text { MC ZZ } \\
\text { Norm. }\end{array}$ & \multicolumn{4}{|c}{$1.0000 \pm 0.0000$} \\
\hline $\begin{array}{l}\geq 4 \text { good } \\
\text { elec. }\end{array}$ & \multicolumn{4}{|c}{$0.2728 \pm 0.0012$} \\
\hline $\begin{array}{l}p_{T} \text { and } \\
\text { overlap } \\
\text { removal }\end{array}$ & \multicolumn{4}{|c}{$0.2292 \pm 0.0011$} \\
\hline & $\begin{array}{c}2 \text { CC/0 ICR } \\
\text { Cumulative } A \times \epsilon\end{array}$ & $\begin{array}{c}3 \text { CC/0 ICR } \\
\text { Cumulative } A \times \epsilon\end{array}$ & $\begin{array}{c}4 \text { CC } / 0 \text { ICR } \\
\text { Cumulative } A \times \epsilon\end{array}$ & $\begin{array}{c}\geq 2 / 1 \text { ICR } \\
\text { Cumulative } A \times \epsilon\end{array}$ \\
\cline { 2 - 5 } $\begin{array}{l}\text { num CC } \\
\text { elec. }\end{array}$ & $0.0242 \pm 0.0004$ & $0.0578 \pm 0.0006$ & $0.0514 \pm 0.0005$ & $0.0745 \pm 0.0006$ \\
\hline mass & $0.0242 \pm 0.0004$ & $0.0578 \pm 0.0006$ & $0.0514 \pm 0.0005$ & $0.0745 \pm 0.0006$ \\
\hline \hline
\end{tabular}


The Acc $\times \epsilon$ values for the various topologies and physics processes examined in this analysis are summarized in Tables 5.28-5.29, where all uncertainties are statistical.

Table 5.28: RunIIa: Acceptance $\times$ efficiency values.

\begin{tabular}{|l|c|c|c|}
\hline Channel & Signal & Migration & $2 e 2 \tau$ \\
\hline eеee $(2 \mathrm{CC})$ & $0.0309 \pm 0.0011$ & $(4.16 \pm 0.61) \times 10^{-5}$ & $(4.41 \pm 1.29) \times 10^{-4}$ \\
\hline eеee $(3 \mathrm{CC})$ & $0.0702 \pm 0.0016$ & $(6.37 \pm 0.71) \times 10^{-5}$ & $(7.13 \pm 1.53) \times 10^{-4}$ \\
\hline eeee $(4 \mathrm{CC})$ & $0.0658 \pm 0.0015$ & $(6.69 \pm 0.72) \times 10^{-5}$ & $(9.33 \pm 1.84) \times 10^{-4}$ \\
\hline eеee $(1 \mathrm{ICR})$ & $0.0901 \pm 0.0018$ & $(6.51 \pm 0.68) \times 10^{-5}$ & $(1.01 \pm 0.19) \times 10^{-3}$ \\
\hline
\end{tabular}

Table 5.29: RunIIb: Acceptance $\times$ efficiency values.

\begin{tabular}{|l|c|c|c|}
\hline Channel & Signal & Migration & $2 e 2 \tau$ \\
\hline eeee $(2 \mathrm{CC})$ & $0.0241 \pm 0.0004$ & $(2.16 \pm 0.27) \times 10^{-4}$ & $(2.14 \pm 0.32) \times 10^{-4}$ \\
\hline eeee $(3 \mathrm{CC})$ & $0.0577 \pm 0.0006$ & $(3.73 \pm 0.20) \times 10^{-4}$ & $(6.11 \pm 0.57) \times 10^{-4}$ \\
\hline eeee $(4 \mathrm{CC})$ & $0.0513 \pm 0.0005$ & $(3.99 \pm 0.19) \times 10^{-4}$ & $(6.78 \pm 0.61) \times 10^{-4}$ \\
\hline eeee $(1 \mathrm{ICR})$ & $0.0744 \pm 0.0006$ & $(3.91 \pm 0.19) \times 10^{-4}$ & $(7.34 \pm 0.64) \times 10^{-4}$ \\
\hline
\end{tabular}

\subsection{2 $\quad \mu \mu \mu \mu$ channel}

The weighted MC cut flow for non-resonant $Z Z \rightarrow \mu \mu \mu \mu$ is shown in Table 5.30.

The $A c c \times \epsilon$ cut flow values for non-resonant $Z Z$ is given in Table 5.31. These results, plus other backgrounds, are summarized in Table 5.32 for the $\mu \mu \mu \mu$ channel. The values for the non-resonant $Z Z$ channel are normalized to the mass $\left(M_{1}\left(Z / \gamma^{*}\right)>\right.$ $\left.30 \mathrm{GeV}, M_{2}\left(Z / \gamma^{*}\right)>30\right)$ region. 
Table 5.30: RunII Cut flow for $\mu \mu \mu \mu$ weighted non-resonant $Z Z$ MC.

\begin{tabular}{l|c|c}
\hline \hline Cut & RunIIa Events & RunIIb Events \\
\hline Initial & 194731 & 1114754 \\
\hline Data Quality & 188157 & 1069667 \\
\hline Z Mass generated $>30 \mathrm{Gev}$ & 17983 & 103545 \\
\hline Trigger & 17983 & 103545 \\
\hline 4 good muons & 8097 & 36575 \\
\hline MounCorr weighted & 6944 & 34752 \\
\hline Track $\Delta Z<3 \mathrm{~cm}$ & 6932 & 33686 \\
\hline Isolation & 5918 & 28363 \\
\hline Muon charges and $p_{T}>15 \mathrm{GeV}$ & 5013 & 23949 \\
\hline Charge & 4915 & 23438 \\
\hline Invariant mass reconstructed $>30 \mathrm{GeV}$ & 4852 & 23109 \\
\hline \hline
\end{tabular}

Table 5.31: Cut flow for RunII $\mu \mu \mu \mu$ non-resonant $Z$ MC showing cumulative and exclusive acceptance times efficiency.

\begin{tabular}{l|c|c}
\hline \hline Cut & RunIIa & RunIIb \\
\hline & Cumulative $A \times \epsilon$ & Cumulative $A \times \epsilon$ \\
\hline MC ZZ Norm. & $1.0000 \pm 0.0000$ & $1.0000 \pm 0.0000$ \\
\hline 4 good muons & $0.4502 \pm 0.0029$ & $0.3532 \pm 0.0011$ \\
\hline Track $\Delta Z$ & $0.3855 \pm 0.0028$ & $0.3253 \pm 0.0011$ \\
\hline Isolation & $0.3291 \pm 0.0027$ & $0.2739 \pm 0.0011$ \\
\hline$p_{T}$ & $0.2787 \pm 0.0026$ & $0.2312 \pm 0.0010$ \\
\hline Charge & $0.2733 \pm 0.0026$ & $0.2263 \pm 0.0010$ \\
\hline Invariant mass & $0.2699 \pm 0.0026$ & $0.2185 \pm 0.0016$ \\
\hline \hline
\end{tabular}

\subsection{3 $е e \mu \mu$ channel}

The $A c c \times \epsilon$ cut flow values for non-resonant $Z Z$ is shown in Tables 5.33 and 5.34 for the $e e \mu \mu$ channel, respectively. These values are normalized to the mass $\left(M_{1}\left(Z / \gamma^{*}\right)>\right.$ $\left.30 \mathrm{GeV}, M_{2}\left(Z / \gamma^{*}\right)>30\right)$ region. The $A c c \times \epsilon$ values for the various process described 
Table 5.32: Acceptance $\times$ efficiency values for the $\mu \mu \mu \mu$ Channel.

\begin{tabular}{l|c|c|c|c}
\hline \hline & Signal & $2 \mu 2 \tau$ & $2 e 2 \tau$ & $4 \tau$ \\
\hline RunIIa & $0.2699 \pm 0.0025$ & $3.925 \mathrm{e}-03 \pm 3.45 \mathrm{e}-04$ & - & $1.4817 \mathrm{e}-05 \pm 2.0883 \mathrm{e}-05$ \\
\hline RunIIb & $0.2185 \pm 0.0016$ & $3.085 \mathrm{e}-03 \pm 2.17 \mathrm{e}-04$ & - & $6.8056 \mathrm{e}-05 \pm 1.8985 \mathrm{e}-05$ \\
\hline \hline & Signal Migration & $2 \mu 2 \tau$ Migration & $4 \tau$ Migration & $t \bar{t}$ \\
\hline RunIIa & $8.222 \mathrm{e}-04 \pm 7.22 \mathrm{e}-05$ & $1.9485 \mathrm{e}-05 \pm 1.1069 \mathrm{e}-05$ & - & $3.9425 \mathrm{e}-07 \pm 5.0872 \mathrm{e}-07$ \\
\hline RunIIb & $6.402 \mathrm{e}-04 \pm 4.50 \mathrm{e}-05$ & $9.1702 \mathrm{e}-06 \pm 3.2315 \mathrm{e}-06$ & - & $7.9372 \mathrm{e}-08 \pm 1.2647 \mathrm{e}-07$ \\
\hline \hline
\end{tabular}

above and other backgrounds are summarized in Tables 5.35-5.38, where all uncertainties are statistical. 
Table 5.33: RunIIa: Cut flow for non-resonant $Z Z \rightarrow e e \mu \mu$ MC background showing cumulative acceptance times efficiency for three subchannels $(=0,=1$ or $\geq 2$ central electrons).

\begin{tabular}{l|c|c|c}
\hline \hline Cut & \multicolumn{3}{|c}{ Cumulative $A \times \epsilon$} \\
\hline $\begin{array}{l}\text { MC ZZ } \\
\text { Norm. }\end{array}$ & \multicolumn{3}{|c}{$1.0000 \pm 0.0000$} \\
\hline $\begin{array}{l}\geq 1 \text { good } \\
\text { elec. }\end{array}$ & \multicolumn{3}{|c}{$0.5507 \pm 0.0029$} \\
\hline $\begin{array}{l}\geq 1 \text { good } \\
\text { muon }\end{array}$ & \multicolumn{3}{|c}{$0.2819 \pm 0.0026$} \\
\hline$p_{T}$ & 0 CC electrons & $\begin{array}{c}1 \text { CC electrons } \\
\text { Cumulative } A \times \epsilon\end{array}$ & 2 CC electrons \\
\hline $\begin{array}{l}\text { num CC } \\
\text { elec. }\end{array}$ & $0.0152 \pm 0.0007$ & $0.0789 \pm 0.0016$ & $0.1503 \pm 0.0021$ \\
\hline$\mu$ cos $\alpha$ and \\
$\mu \mu$ acopla- \\
$\begin{array}{l}\text { narity } \\
\text { cut }\end{array}$ & $0.0151 \pm 0.0007$ & $0.0789 \pm 0.0016$ & $0.1502 \pm 0.0021$ \\
\hline$\mu$ track $\Delta z$ & $0.0151 \pm 0.0007$ & $0.0787 \pm 0.0015$ & $0.1502 \pm 0.0022$ \\
\hline$\Delta R_{e \mu}$ & $0.0148 \pm 0.0007$ & $0.0756 \pm 0.0015$ & $0.1398 \pm 0.0020$ \\
\hline mass & $0.0147 \pm 0.0007$ & $0.0755 \pm 0.0015$ & $0.1395 \pm 0.0020$ \\
\hline \hline
\end{tabular}


Table 5.34: RunIIb: Cut flow for non-resonant $Z Z \rightarrow e e \mu \mu$ MC background showing cumulative times efficiency for three subchannels $(=0,=1$ or $\geq 2$ central electrons).

\begin{tabular}{|c|c|c|c|}
\hline 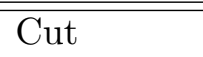 & \multicolumn{3}{|c|}{ Cumulative $A \times \epsilon$} \\
\hline $\begin{array}{l}\text { MC ZZ } \\
\text { Norm. }\end{array}$ & \multicolumn{3}{|c|}{$1.0000 \pm 0.0000$} \\
\hline $\begin{array}{l}\geq 1 \text { good } \\
\text { elec. }\end{array}$ & \multicolumn{3}{|c|}{$0.4768 \pm 0.0012$} \\
\hline $\begin{array}{l}\geq 1 \text { good } \\
\text { muon }\end{array}$ & \multicolumn{3}{|c|}{$0.2517 \pm 0.0011$} \\
\hline$p_{T}$ & \multicolumn{3}{|c|}{$0.1977 \pm 0.0010$} \\
\hline \multirow[b]{2}{*}{$\begin{array}{l}\text { num CC } \\
\text { elec. }\end{array}$} & $\begin{array}{c}0 \text { CC electrons } \\
\text { Cumulative } A \times \epsilon\end{array}$ & $\begin{array}{c}1 \text { CC electrons } \\
\text { Cumulative } A \times \epsilon\end{array}$ & $\begin{array}{c}2 \text { CC electrons } \\
\text { Cumulative } A \times \epsilon\end{array}$ \\
\hline & $0.0111 \pm 0.0003$ & $0.0660 \pm 0.0006$ & $0.1201 \pm 0.0008$ \\
\hline $\begin{array}{l}\mu \cos \alpha \text { and } \\
\mu \mu \text { acopla- } \\
\text { narity } \\
\text { cut }\end{array}$ & $0.0111 \pm 0.0003$ & $0.0660 \pm 0.0006$ & $0.1199 \pm 0.0008$ \\
\hline$\mu$ track $\Delta z$ & $0.0109 \pm 0.0003$ & $0.0654 \pm 0.0006$ & $0.1189 \pm 0.0008$ \\
\hline$\Delta R_{e \mu}$ & $0.0107 \pm 0.0002$ & $0.0616 \pm 0.0006$ & $0.1059 \pm 0.0007$ \\
\hline mass & $0.0107 \pm 0.0002$ & $0.0614 \pm 0.0006$ & $0.1057 \pm 0.0007$ \\
\hline
\end{tabular}

\begin{tabular}{|l|c|c|c|}
\hline Channel & Signal & Migration & $t \bar{t}$ \\
\hline$e e \mu \mu(0 \mathrm{CC})$ & $0.014 \pm 0.001$ & $\left(4.66_{-3.27}^{+6.35}\right) \times 10^{-6}$ & $\left(1.00_{-1.00}^{+1.00}\right) \times 10^{-7}$ \\
\hline$e e \mu \mu(1 \mathrm{CC})$ & $0.076 \pm 0.002$ & $\left(5.70_{-1.89}^{+2.43}\right) \times 10^{-5}$ & $\left(1.05_{-0.73}^{+1.42}\right) \times 10^{-6}$ \\
\hline$e e \mu \mu(2 \mathrm{CC})$ & $0.140 \pm 0.002$ & $\left(8.68_{-4.09}^{+6.02}\right) \times 10^{-6}$ & $\left(5.35_{-3.73}^{+7.26}\right) \times 10^{-7}$ \\
\hline
\end{tabular}

Table 5.35: RunIIa: Acceptance $\times$ efficiency values.

\begin{tabular}{|l|c|c|c|}
\hline Channel & $2 \mu 2 \tau$ & $2 e 2 \tau$ & $4 \tau$ \\
\hline$e е \mu \mu(0 \mathrm{CC})$ & $(0.30 \pm 0.10) \times 10^{-3}$ & $(0.25 \pm 0.09) \times 10^{-3}$ & - \\
\hline$e е \mu \mu(1 \mathrm{CC})$ & $(0.87 \pm 0.16) \times 10^{-3}$ & $(0.66 \pm 0.14) \times 10^{-3}$ & - \\
\hline$e e \mu \mu(2 \mathrm{CC})$ & $(1.45 \pm 0.21) \times 10^{-3}$ & $(1.98 \pm 0.24) \times 10^{-3}$ & - \\
\hline
\end{tabular}

Table 5.36: RunIIa: Acceptance $\times$ efficiency values. 


\begin{tabular}{|l|c|c|c|}
\hline Channel & Signal & Migration & $t \bar{t}$ \\
\hline$е e \mu \mu(0 \mathrm{CC})$ & $0.011 \pm 0.001$ & $(2.25 \pm 0.66) \times 10^{-5}$ & $\left(2.95_{-2.06}^{+4.00}\right) \times 10^{-6}$ \\
\hline$е e \mu \mu(1 \mathrm{CC})$ & $0.061 \pm 0.001$ & $(4.72 \pm 0.91) \times 10^{-5}$ & $\left(1.31_{-0.51}^{+0.68}\right) \times 10^{-6}$ \\
\hline$e е \mu \mu(2 \mathrm{CC})$ & $0.104 \pm 0.001$ & $(5.07 \pm 0.99) \times 10^{-5}$ & $\left(3.29_{-1.26}^{+2.00}\right) \times 10^{-7}$ \\
\hline
\end{tabular}

Table 5.37: RunIIb: Acceptance $\times$ efficiency values.

\begin{tabular}{|l|c|c|c|}
\hline Channel & $2 \mu 2 \tau$ & $2 e 2 \tau$ & $4 \tau$ \\
\hline$e е \mu \mu(0 \mathrm{CC})$ & $(1.70 \pm 0.30) \times 10^{-4}$ & $(9.31 \pm 2.95) \times 10^{-5}$ & - \\
\hline$e е \mu \mu(1 \mathrm{CC})$ & $(6.63 \pm 0.60) \times 10^{-4}$ & $(7.63 \pm 0.83) \times 10^{-4}$ & - \\
\hline$e е \mu \mu(2 \mathrm{CC})$ & $(1.81 \pm 0.10) \times 10^{-3}$ & $(1.30 \pm 0.11) \times 10^{-3}$ & - \\
\hline
\end{tabular}

Table 5.38: RunIIb: Acceptance $\times$ efficiency values. 


\subsection{Z + jets/QCD Background}

$Z+$ jets background arises in cases where there is a $Z(\rightarrow \ell \ell)$ with $\geq 1$ jets in the final state and the jets mimic an electron or contain a muon. In the case of a mimicked electron,

a jet is falsely identified as an electron. In the case of a muon, the jet usually contains a real muon from in-flight decays of pions, kaons, or a heavy quark. $Z(\rightarrow \ell \ell)+\gamma+$ jets production where a photon is also mis-identified as an electron primarily contaminates eeee and $е e \mu \mu$ channels.

To calculate the $\mathrm{Z}+$ jets background, first the probability for a jet to be mis-identified as a lepton (fakerate) for each type of lepton (electron or muon) is determined. This is found by processing the appropriate $\mathrm{Z}+$ jets sample. For RunIIa a specific sample that contains 98,176,223 events. For RunIIb a different sample is used that contains 121,792,353 events. Once the fakerates are calculated, the $\mathrm{Z}+$ jets background is determined by running over the signal data sample for each channel and applying an appropriate cut flow. Details of how the fakerates and backgrounds are determined are described in the next sections.

\subsubsection{Electron fakerate and $\mathrm{Z}+$ jets background determination in eeee channel}

The electron fakerate (per jet) is determined using a tag and probe method. First, we find all "good" jets [36] that have $p_{T}>15 \mathrm{GeV}$. We require that the event has two such jets. To find the tag jet, we apply the following additional criteria:

- Energy fraction in the electromagnetic calorimeter $\left(f_{E M}\right): 0.05<f_{E M}<0.95$ 
- Energy fraction in the coarse hadronic part of the calorimeter $\left(f_{C H}=\frac{E_{C H}}{E_{t o t}}\right): f_{C H}<$ 0.4

- number of calorimeter towers that represent $90 \%$ of the total jet energy $(n 90)<20$

The remaining jet is the probe jet. We then additionally require that the $\Delta \phi$ between the tag and probe jets be $>3.0$ radians. At this point we reject events with missing transverse energy $\left(\mathscr{E}_{T}\right)<20 \mathrm{GeV}$. This is to suppress possible $W+$ jet contamination, which would contribute by increasing the fakerate to larger than expected values.

We find all good electrons in the event as defined previously with a $p_{T}$ requirement of $15 \mathrm{GeV}$. We look for cases where the $\Delta \phi$ between the electron and the probe jet is $>3.0$ which means the jet is mimicking an electron. In this case, we fill both our numerator and denominator histograms with the probe jet information in two-dimensions of $\eta_{\text {det }}$ and $p_{T}$. If this $\Delta R$ cut fails, we fill only the denominator plot. The fakerate is then the efficiency as found by dividing the numerator plots by the denominator plots. Obtained electron fake rate results are shown in Fig. A.1-A.2 in Appendix A.

In addition to the background from a jet faking an electron in the $\mathrm{CC}$ or $\mathrm{EC}$, we also may have events where a jet fakes an electron in the ICR. We model this background using the same method as outlined above, except in this case we determine the fake rate using probe jets within the ICR region, and apply the fake rate to events with three good $\mathrm{CC} / \mathrm{EC}$ electrons and a jet in the ICR region. In the ICR region, the energy resolution is poorer, so we first examined the fake rate versus $\mathbb{E}_{T}$ to determine where to cut to remove $W$ contamination. We apply the standard Jet Energy Scale (JES) correction [38] for the jet in the ICR when determining the $\mathbb{E}_{T}$ for the event, unless the jet passes all of our signal ICR cuts. It has previously been found [39] that the JES correction overestimates the energy of ICR electron objects. We instead substitute the $p_{T}$ of the track for the calorimeter energy of the ICR object in the $\mathbb{E}_{T}$ calculation for these events. All other objects in the event 
enter the $\mathbb{E}_{T}$ calculation normally. We find that an $\mathbb{E}_{T}$ cut of $20 \mathrm{GeV}$ also works well in the ICR region (Fig. A.3). The fake rates after this cut versus $p_{T}$ and $\eta_{D}$ are shown in Fig. A.4 and A.5 for Run IIa and Run IIb respectively. All these fake rates are also shown in Appendix A.

The background is then calculated by running over the 2EMhighpt data sample and selecting events with good electrons and jets that are in the CC, ICR, or EC region. We apply the following cut flow:

- at least three electrons (to correctly account for $Z+\gamma+$ jets background)

- electrons must satisfy $p_{T}^{1}>15.0 \mathrm{GeV}, p_{T}^{2}>15.0 \mathrm{GeV}, p_{T}^{3}>15.0 \mathrm{GeV}$

- at least one jet with $\Delta R>0.5$ with respect to the three electrons that also passes $p_{T}>15.0 \mathrm{GeV}$

- loop over all jets passing above cut, requiring that the combination of each jet and the three electrons satisfy the normal $p_{T}$ cuts of the signal selection, and sum up all of the fakerate values for each jet

- Split sample into four subchannels, corresponding to $N_{C C E M}=2,3,4 ; N_{I C R E M}=0$ and $N_{C C E M} \geq 2 ; N_{I C R E M}=1$

- pass the $Z$ mass requirement of one pair with a dimass $>30 \mathrm{GeV}$ and the other with a dimass $>30 \mathrm{GeV}$.

The resulting $\mathrm{Z}+$ jets background for the three sub-channels and both run epochs are shown in Tables 5.39 and 5.40. 
Table 5.39: RunIIa estimated $\mathrm{Z}+$ jets background in three eeee subchannels for $3 e+$ jet, $2 e+$ jet and total event yield, where total is $3 e+$ jet minus $2 e+$ jet.

\begin{tabular}{c|c|c|c}
\hline \hline Number of CC objects & $3 e+$ jet & $2 e+$ jet & Total \\
\hline$=2$ & $1.69_{-0.40}^{+0.47}\left(\times 10^{-2}\right)$ & $6.69 \pm 0.10\left(\times 10^{-4}\right)$ & $1.62_{-0.40}^{+0.47}\left(\times 10^{-2}\right)$ \\
\hline$=3$ & $0.71_{-0.24}^{+0.30}\left(\times 10^{-2}\right)$ & $4.93 \pm 0.07\left(\times 10^{-4}\right)$ & $0.66_{-0.24}^{+0.30}\left(\times 10^{-2}\right)$ \\
\hline$\geq 4$ & $3.29_{-1.38}^{+1.93}\left(\times 10^{-3}\right)$ & $2.68 \pm 0.05\left(\times 10^{-4}\right)$ & $3.02_{-1.38}^{+1.93}\left(\times 10^{-3}\right)$ \\
\hline \hline
\end{tabular}

Table 5.40: RunIIb estimated Z + jets background in three eeee subchannels for $3 e+$ jet, $2 e+$ jet and total event yield, where total is $3 e+$ jet minus $2 e+$ jet.

\begin{tabular}{c|c|c|c}
\hline \hline Number of CC objects & $3 e+$ jet & $2 e+$ jet & Total \\
\hline$=2$ & $5.39 \pm 0.18\left(\times 10^{-2}\right)$ & $5.16 \pm 0.03\left(\times 10^{-3}\right)$ & $4.87 \pm 0.18\left(\times 10^{-2}\right)$ \\
\hline$=3$ & $3.76 \pm 0.14\left(\times 10^{-2}\right)$ & $3.67 \pm 0.02\left(\times 10^{-3}\right)$ & $3.39 \pm 0.14\left(\times 10^{-2}\right)$ \\
\hline$\geq 4$ & $2.30_{-0.48}^{+0.55}\left(\times 10^{-2}\right)$ & $2.11 \pm 0.02\left(\times 10^{-3}\right)$ & $2.09_{-0.48}^{+0.55}\left(\times 10^{-2}\right)$ \\
\hline \hline
\end{tabular}

\subsubsection{Muon fakerate and $\mathbf{Z}+$ jets background determination in $\mu \mu \mu \mu$ channel}

The muon fakerate (per jet) is determined using the same tag and probe method as described in the previous section for the electron fakerate. The good muons are broken up into six groups depending on $p_{T}(15 \mathrm{GeV}, 25 \mathrm{GeV}$ or $30 \mathrm{GeV})$ and isolation. For each group we look for cases where the $\Delta R$ between the muon and the probe jet is $<0.5$ which means the jet contains a muon. In this case, we fill both our numerator and denominator plots with the probe jet $p_{T}$ and the probe jet $\eta_{\text {det }}$ in two-dimensions. If the $\Delta R$ cut fails, we fill only the denominator plot. The fakerate is then the efficiency as found by dividing the numerator plots by the denominator plots in the six groups. The obtained muon fake rate 
results are shown in Figures B.1-B.4 in Appendix B. For reference, the average fakerate for RunIIb muons with $p_{T}>15 \mathrm{GeV}$, where only nseg $=0$ and 1 muons are isolated is $0.0039 \pm 0.003$, while the average is $0.0017 \pm 0.0001$ for the same muon $p_{T}$ but all the muons are required to be isolated.

The background is then calculated by running over the 2MUhighpt data sample and selecting events with good muons and jets. We apply the following cut flow:

- at least two muons

- muons satisfy $p_{T}^{1}>15 \mathrm{GeV}, p_{T}^{2}>15 \mathrm{GeV}$

- both muons must be isolated

- $\cos \alpha<0.96$ between muon pair

- $\Delta z<3.0 \mathrm{~cm}$ between muon tracks

- at least two jets with $\Delta R>0.5$ with respect to the two muons that also pass $p_{T}>$ $15.0 \mathrm{GeV}$

- loop over all jets passing above cut, and sum up all of the fakerate values for each jet Note that no $Z$ mass cut is applied since the jet kinematics are not the same as those for the muon.

The resulting RunIIa $\mathrm{Z}+$ jets background is $0.0028 \pm 0.0001$ events. The resulting RunIIb $\mathrm{Z}+$ jets background is $0.0161 \pm 0.0002$ events.

\subsection{3 $е e \mu \mu \mathbf{Z}+$ jets Background}

This channel uses the fakerates determined for electrons and muons as described previously. Two different contributions are determined: 
1. Two muons plus an electron plus a jet, where we apply the electron fakerate to the jet. This method gives us an estimate of a background due to $Z(\rightarrow \mu \mu)+$ jets and $Z(\rightarrow \mu \mu)+\gamma+$ jets where a jet or a photon can mimic an electron.

2. Two electrons plus two jets, where we apply the muon fakerate to the jets. This method gives us an estimate of the background due to $Z(\rightarrow e e)+$ jets where jets can contain muons.

In the first case, the background is determined by running over the 2MUhighpt data sample and finding good muons and electrons in the event as defined previously and any good jets in the event. We then apply the following cut flow:

- at least two muons and one electron

- muons satisfy $p_{T}^{1}>25.0 \mathrm{GeV}, p_{T}^{2}>15.0 \mathrm{GeV}$ and electron satisfies $p_{T}^{1}>15.0 \mathrm{GeV}$

- at least one isolated muon

- require $\cos \alpha<0.96$ between muon pair

- require $\Delta z<3.0 \mathrm{~cm}$ between muon tracks

- require $\Delta R$ between electron and muons to be $>0.2$

- at least one jet with $\Delta R>0.5$ with respect to the muons and electron that also passes $p_{T}>15.0 \mathrm{GeV}$ and is either $\mathrm{CC}$ or $\mathrm{EC}$

- perform a cut on the number of CC objects $(=0,=1, \geq 2)$ combining the electron with the jets

- loop over all jets passing the above cut, requiring that the combination of each jet and the electron satisfy the normal $p_{T}$ cuts of the signal selection, and sum up all of the fakerate values for each jet 
- pass the $Z$ mass requirement of one pair with a dimass $>30 \mathrm{GeV}$ and the other with a dimass $>30 \mathrm{GeV}$ where one pair is the $\mu \mu$ and the other is the $e j$ mass with the leading jet

While this method correctly takes into account contribution from $Z+\gamma+$ jets production (and other smaller possible contributions such as $W Z+$ jets, $W W+$ jets, $W+$ jets, $\geq 4$ jets), it double counts $Z \rightarrow \mu \mu+$ jets background. An event with two muons and two jets enters the two muons + electron + jet sample if either of the jets is misidentified as an

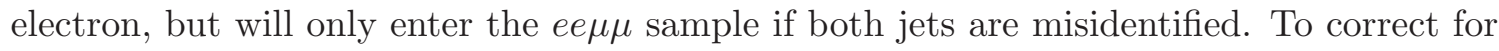
this effect, we estimate the $Z \rightarrow \mu \mu+$ jets contribution separately (with no lumped $Z+\gamma$ + jets contribution) by selecting a two muons plus two jets sample, applying the similar cut flow as described above, and applying the electron fakerate to both jets. We subtract the resulting estimate from the one obtained using the two muons plus electron plus jets sample.

In the second case, the background is determined by running over the 2EMhighpt data sample and finding good electrons in the event as defined previously and any good jets in the event. We then apply the following cut flow:

- at least two electrons

- electrons satisfy $p_{T}^{1}>25 \mathrm{GeV}, p_{T}^{2}>15 \mathrm{GeV}$

- perform a cut on the number of CC electrons $(=0,=1, \geq 2)$

- at least two jets with $\Delta R>0.5$ with respect to the two electrons that also pass $p_{T}>15.0 \mathrm{GeV}$

- loop over all jets passing above cut, considering the $15 \mathrm{GeV}$ and $25 \mathrm{GeV}$ combinations of muon $p_{T}$ and isolation fakerate groups 
- require $M_{e e}>50 \mathrm{GeV}$.

The final background is the sum of the two cases as shown in Tables 5.41-5.42.

Table 5.41: RunIIa $Z+$ jets background for $e e \mu \mu$ channel for the three cases of $=0,=1$ or $\geq 2$ CC objects (electron+jet) showing the $2 \mu+e+$ jet, $2 \mu+2$ jet, $2 e+2$ jet and total event yield, where the total is $2 \mu+e+$ jet minus $2 \mu+2$ jet plus $2 e+2$ jet.

\begin{tabular}{c|c|c|c|c}
\hline \hline CC objects & $2 \mu+e+$ jet & $2 \mu+2$ jet & $2 e+2$ jet & Total \\
\hline$=0$ & $3.47_{-1.91}^{+3.06}\left(\times 10^{-3}\right)$ & $1.11 \pm 0.06\left(\times 10^{-4}\right)$ & $1.57 \pm 0.05\left(\times 10^{-3}\right)$ & $4.93_{-1.91}^{+3.06}\left(\times 10^{-3}\right)$ \\
\hline$=1$ & $6.70_{-2.22}^{+2.86}\left(\times 10^{-3}\right)$ & $3.53 \pm 0.08\left(\times 10^{-4}\right)$ & $3.35 \pm 0.04\left(\times 10^{-3}\right)$ & $9.70_{-2.22}^{+2.86}\left(\times 10^{-3}\right)$ \\
\hline$\geq 2$ & $9.40_{-2.94}^{+3.73}\left(\times 10^{-3}\right)$ & $6.28 \pm 0.10\left(\times 10^{-4}\right)$ & $3.47 \pm 0.06\left(\times 10^{-3}\right)$ & $1.22_{-0.29}^{+0.37}\left(\times 10^{-2}\right)$ \\
\hline \hline
\end{tabular}

Table 5.42: RunIIb $Z+$ jets background for $e e \mu \mu$ channel for the three cases of $=0,=1$ or $\geq 2 \mathrm{CC}$ objects (electron + jet) showing the $2 \mu+e+$ jet, $2 \mu+2$ jet, $2 e+2$ jet and total event yield, where the total is $2 \mu+e+$ jet minus $2 \mu+2$ jet plus $2 e+2$ jet.

\begin{tabular}{c|c|c|c|c}
\hline \hline CC objects & $2 \mu+e+$ jet & $2 \mu+2$ jet & $2 e+2$ jet & Total \\
\hline$=0$ & $1.98_{-0.56}^{+0.69}\left(\times 10^{-2}\right)$ & $0.68 \pm 0.02\left(\times 10^{-3}\right)$ & $5.70 \pm 0.04\left(\times 10^{-3}\right)$ & $2.48_{-0.56}^{+0.69}\left(\times 10^{-2}\right)$ \\
\hline$=1$ & $3.72 \pm 0.26\left(\times 10^{-2}\right)$ & $2.16 \pm 0.04\left(\times 10^{-3}\right)$ & $1.18 \pm 0.01\left(\times 10^{-2}\right)$ & $4.68 \pm 0.26\left(\times 10^{-2}\right)$ \\
\hline$\geq 2$ & $6.02 \pm 0.19\left(\times 10^{-2}\right)$ & $5.26 \pm 0.06\left(\times 10^{-3}\right)$ & $1.08 \pm 0.01\left(\times 10^{-2}\right)$ & $6.57 \pm 0.19\left(\times 10^{-2}\right)$ \\
\hline \hline
\end{tabular}

\section{6 t $t \bar{t}$ Background}

Top pair production can lead to final states with four leptons. This background is estimated using $t \bar{t} \rightarrow 2 b+2 \ell+2 \nu$ MC events. We use a cross section value of $\sigma^{N N L L}(t \bar{t})=7.9 \mathrm{pb}[40]$ assuming $m_{t o p}=170 \mathrm{GeV}$, which gives us $\sigma^{N N L L}(t \bar{t}) \times B R^{2}(W \rightarrow \ell)=7.9 \times 0.321^{2}=814 \mathrm{fb}$ for the normalization. The acceptance times efficiency values for $t \bar{t} \rightarrow 2 b+2 \ell+2 \nu$ can be 
found in the Monte Carlo section of this note. A small contamination is observed only in the $e e \mu \mu$ channel.

\subsection{Beam Halo and Cosmic Ray Muon Background}

Beam halo interactions and cosmic ray muons overlaying on top of physics processes such as $W Z \rightarrow \mu \mu \mu, W Z \rightarrow \mu e e, Z \rightarrow \mu \mu, Z \rightarrow e e, W W \rightarrow \mu \mu$, or $W W \rightarrow$ ee can produce events containing four muons or two muons and two electrons. To estimate the contribution due to beam halo and cosmics, we select events with four muons applying our nominal selection cut flow, but removing requirements on muon dca, timing, and $\Delta z<3$ cm between muon pairs. For RunIIa we see 1361 candidates in our data, which we assume to be dominated by beam halo and cosmic ray muons contributions. For RunIIb we see 43480 candidate events.

We estimate rejection factors of muon dca, timing and $\Delta z<3 \mathrm{~cm}$ cuts from a sample where we apply the selection described above, but also relaxing $p_{T}$ cuts on muons from the nominal $15 \mathrm{GeV}, 15 \mathrm{GeV}, 15 \mathrm{GeV}, 15 \mathrm{GeV}$ to $10 \mathrm{GeV}, 10 \mathrm{GeV}, 5 \mathrm{GeV}, 5 \mathrm{GeV}$. Then the rejection factor of each of the three requirements is estimated in a subsample where the two other requirements are reversed. The results are given in Tables 5.43 and 5.44. We use one event instead of zero passing the dca cut in the sample where timing and $\Delta z<3 \mathrm{~cm}$ are reversed to get a finite estimate for the rejection.

We obtain a total RunIIa rejection factor of $627 \times 3.8 \times 4380=1.04 \times 10^{7}$. Applying this to the 1988 RunIIa events observed in the sample dominated by beam halo and cosmics, we get a conservative estimate of $1.90 \times 10^{-4}$ events. We obtain a total RunIIb rejection factor of $1843 \times 1.5 \times 1204=3.33 \times 10^{6}$. Applying this to the 43480 RunIIb events observed in the sample dominated by beam halo and cosmics, we get a conservative estimate of 0.01 
events. Assuming lepton universality the resulting background from cosmic rays in the $\mu \mu \mu \mu$ and $2 \mu 2 e$ samples are less than 0.01 events for each channel. While this background is smaller than the $Z+$ jets background, its inclusion was tested and found to have a negligible effect on any result for this analysis.

Table 5.43: RunIIa: Rejection factors against beam halo and cosmics from dca, timing and $\Delta z<3 \mathrm{~cm}$ requirements.

\begin{tabular}{l|c|c|c}
\hline \hline Requirement & $N_{\text {initial }}$ & $N_{\text {pass }}$ & Rejection \\
\hline dca & 4387 & 7 & 627 \\
\hline$\Delta z$ & 4380 & $0(1)$ & 4380 \\
\hline \hline
\end{tabular}

Table 5.44: RunIIb: Rejection factors against beam halo and cosmics from dca, timing and $\Delta z<3 \mathrm{~cm}$ requirements.

\begin{tabular}{l|c|c|c}
\hline \hline Requirement & $N_{\text {initial }}$ & $N_{\text {pass }}$ & Rejection \\
\hline dca & 86627 & 47 & 1843 \\
\hline$\Delta z$ & 86652 & 72 & 1204 \\
\hline \hline
\end{tabular}

\subsection{Migration or Misrecronstruction}

This source of contribution to the background arises from the same $Z / \gamma^{*} Z / \gamma^{*} \rightarrow$ $\ell^{+} \ell^{-} \ell^{\prime}+\ell^{\prime}-$ events as our signal, but from low mass $Z / \gamma^{*}$ pair production. In the eeee and $\mu \mu \mu \mu$ channels these events pass the reconstructed $Z$ mass requirement of $>30 \mathrm{GeV}$ and $>30 \mathrm{GeV}$ because the final state involves leptons of the same flavor. Since we do not consider the charge of the leptons, except in the $\mu \mu \mu \mu$ channel, there are three possible $Z Z$ pairs that can be formed. Wrong pairings (leptons wrongly assigned to $Z$ s) from low mass $Z / \gamma^{*} Z / \gamma^{*}$ events can pass mass requirements and contribute into our signal. Momentum 
resolution also allows for events to be misreconstructed in all three channels. This contribution is measured by finding the acceptance times efficiency from the MC signal sample, where at least one of $Z$ s fails the $Z$ mass cut. The results for the various subchannels are given in Chap 6. 


\section{CHAPTER 6 SELECTION RESULTS AND CROSS SECTION CALCULATION}

For the eeee channel, we observe five events, with Higgs boson production expected to give 0.034 events for $m_{H}=125 \mathrm{GeV}$ and 0.068 events for $m_{H}=180 \mathrm{GeV}$. Estimated background amounts to 4.83 events. Details are given in Table 6.1. In the $\mu \mu \mu \mu$ channel three data events are observed with expected 0.040 Higgs boson events for $m_{H}=125$ $\mathrm{GeV}$ and 0.077 events for $m_{H}=180 \mathrm{GeV}$. The total estimated background in this case is 4.41 events. A summary of these results is show in Table 6.2. We also observe five $e e \mu \mu$ candidate events, with expected 0.062 Higgs boson events for $m_{H}=125 \mathrm{GeV}$ and 0.112 events for $m_{H}=180 \mathrm{GeV}$. Total estimated background is 7.55 events. Details of these events are given in Table 6.3. From the three tables we see that the primary background in all channels comes from non-resonant $Z Z$ production. Systematic errors in all aforementioned tables are discussed in Chap 7.

We combine together the three channels to obtain our final results. At the same that non-resonant $Z Z$ component is a background for Higgs production, it can also be used to calculate the $Z Z$ production cross section.

To extract the $Z Z$ production cross section measurement we define the following likelihood function: 
Table 6.1: Contributions from non-negligible backgrounds in the eeee subchannels, expected (Exp.) non-resonant $Z Z$ and Higgs boson signals, and number of observed events. Uncertainties are statistical followed by systematic.

\begin{tabular}{l|c|c|c|c}
\hline \hline & $\begin{array}{l}2 \mathrm{CC} \\
2 \mathrm{EC}\end{array}$ & $\begin{array}{c}3 \mathrm{CC} \\
1 \mathrm{EC}\end{array}$ & $4 \mathrm{CC}$ & $\begin{array}{c}\geq \mathrm{CC} \\
1 \mathrm{ICR}\end{array}$ \\
\hline $\begin{array}{l}\text { QCD backg. } \\
\text { Migration }\end{array}$ & $\begin{array}{c}0.15 \pm 0.01 \pm 0.03 \\
0.014 \pm 0.001 \pm 0.002\end{array}$ & $\begin{array}{c}0.12 \pm 0.01 \pm 0.02 \\
0.023 \pm 0.001 \pm 0.004\end{array}$ & $\begin{array}{c}0.05 \pm 0.01 \pm 0.01 \\
0.025 \pm 0.001 \pm 0.004\end{array}$ & $\begin{array}{c}0.29 \pm 0.024_{-0.12}^{+0.03} \\
0.024 \pm 0.001 \pm 0.003\end{array}$ \\
\hline $\begin{array}{l}\text { Total non- } Z Z \\
\text { background }\end{array}$ & $0.17 \pm 0.01 \pm 0.03$ & $0.15 \pm 0.01 \pm 0.02$ & $0.09 \pm 0.01 \pm 0.01$ & $0.33 \pm 0.04_{-0.12}^{+0.03}$ \\
\hline $\begin{array}{l}\text { Exp. } \\
\text { non-res. } Z Z\end{array}$ & $0.48 \pm 0.01 \pm 0.07$ & $1.14 \pm 0.01 \pm 0.17$ & $1.03 \pm 0.01 \pm 0.15$ & $1.47 \pm 0.01 \pm 0.19$ \\
\hline $\begin{array}{l}\text { Exp. } g g \rightarrow H \\
M_{H}=125 \mathrm{GeV}\end{array}$ & $<0.001$ & 0.001 & 0.004 & 0.002 \\
$\begin{array}{l}\text { Exp. } Z H \\
M_{H}=125 \mathrm{GeV}\end{array}$ & 0.003 & 0.006 & 0.010 & 0.008 \\
\hline $\begin{array}{l}\text { Total Higgs } \\
M_{H}=125 \mathrm{GeV}\end{array}$ & 0.003 & 0.007 & 0.014 & 0.010 \\
\hline $\begin{array}{l}\text { Observed } \\
\text { Events }\end{array}$ & 0 & 1 & 2 & 2 \\
\hline \hline
\end{tabular}


Table 6.2: Contributions from non-negligible backgrounds in the $\mu \mu \mu \mu$ channel, expected non-resonant $Z Z$ and Higgs boson signal, and number of observed events. Uncertainties are statistical followed by systematic.

\begin{tabular}{l|c}
\hline \hline & Number of Events \\
\hline QCD backg. & $0.12 \pm 0.01_{-0.05}^{+0.07}$ \\
Migration & $\left(0.34 \pm 0.02_{-0.04}^{+0.07}\right) \times 10^{-1}$ \\
Cosmic rays & $<0.01$ \\
\hline $\begin{array}{l}\text { Total non- } Z Z \\
\text { background }\end{array}$ & $0.19 \pm 0.01_{-0.05}^{+0.07}$ \\
\hline $\begin{array}{l}\text { Expected } \\
\text { non-resonant } Z Z\end{array}$ & $4.26 \pm 0.02 \pm 0.43$ \\
\hline $\begin{array}{l}\text { Expected } g g \rightarrow H \\
M_{H}=125 \mathrm{GeV}\end{array}$ & 0.007 \\
$\begin{array}{l}\text { Expected } Z H \\
M_{H}=125 \mathrm{GeV}\end{array}$ & 0.033 \\
\hline $\begin{array}{l}\text { Total Higgs boson } \\
M_{H}=125 \mathrm{GeV}\end{array}$ & 0.040 \\
\hline Observed Events & 3 \\
\hline \hline
\end{tabular}

$$
L\left(\sigma,\left\{N_{j}^{o b s}, N_{j}^{b k g d}, \mathcal{B}_{j}, \mathcal{L}_{j}, A c c_{j}\right\}\right)=\prod_{j=1}^{j=14} \mathcal{P}\left(N_{j}^{o b s}, \mu_{j}\right)=\prod_{j=1}^{j=14} \frac{\mu_{j}^{N^{o b s}}}{N_{j}^{o b s !}} e^{-\mu_{j}}
$$

where $\mathcal{P}\left(N_{j}^{o b s}, \mu_{j}\right)$ is the Poisson probability of observing $N_{j}^{o b s}$ events given an expected signal and background yield of: 
Table 6.3: Contributions from non-negligible backgrounds in the ee $\mu \mu$ subchannels, plus expected signal and number of observed events. Uncertainties are statistical followed by systematic.

\begin{tabular}{|c|c|c|c|}
\hline & $0 \mathrm{CC}$ & $1 \mathrm{CC}$ & $2 \mathrm{CC}$ \\
\hline $\begin{array}{l}\text { QCD backg. } \\
t \bar{t} \\
\text { Migration } \\
\text { Cosmic rays }\end{array}$ & $\begin{array}{c}0.11 \pm 0.01 \pm 0.03 \\
\left(0.2_{-0.1}^{+0.3} \pm 0.6\right) \times 10^{-2} \\
\left(2.1_{-0.7}^{+0.9}{ }_{-1.0}^{+0.3}\right) \times 10^{-3} \\
<0.001\end{array}$ & $\begin{array}{c}0.21 \pm 0.01 \pm 0.04 \\
\left(1.0_{-0.3}^{+0.5} \pm 0.2\right) \times 10^{-2} \\
\left(5.0 \pm 0.8_{-1.4}^{+0.6}\right) \times 10^{-3} \\
<0.003\end{array}$ & $\begin{array}{c}0.27 \pm 0.01 \pm 0.04 \\
\left(0.3_{-0.1}^{+0.2} \pm 0.3\right) \times 10^{-2} \\
\left(4.8_{-0.5}^{+0.6} \pm 1.0\right) \times 10^{-3} \\
<0.006\end{array}$ \\
\hline $\begin{array}{l}\text { Total non- } Z Z \\
\text { background }\end{array}$ & $0.12 \pm 0.01 \pm 0.03$ & $0.25 \pm 0.01 \pm 0.04$ & $0.31 \pm 0.01 \pm 0.04$ \\
\hline $\begin{array}{l}\text { Expected } \\
\text { non-resonant } Z Z\end{array}$ & $0.43 \pm 0.01 \pm 0.06$ & $2.37 \pm 0.02 \pm 0.28$ & $4.13 \pm 0.03 \pm 0.49$ \\
\hline $\begin{array}{l}\text { Expected } g g \rightarrow H \\
M_{H}=125 \mathrm{GeV}\end{array}$ & $<0.001$ & 0.002 & 0.007 \\
\hline $\begin{array}{l}\text { Expected } Z H \\
M_{H}=125 \mathrm{GeV}\end{array}$ & 0.001 & 0.015 & 0.036 \\
\hline $\begin{array}{l}\text { Total Higgs boson } \\
M_{H}=125 \mathrm{GeV}\end{array}$ & 0.002 & 0.017 & 0.043 \\
\hline Observed Events & 2 & 1 & 2 \\
\hline
\end{tabular}

$$
\mu_{j}=\sigma \times A c c_{j} \times \mathcal{B}_{j} \times \mathcal{L}_{j}+N_{j}^{b k g d}
$$

Here, $A c c_{j}$ is the acceptance times efficiency, $\mathcal{L}_{j}$ is the luminosity, $\mathcal{B}_{j}$ is the branching fraction and $N_{j}^{b k g d}$ is the expected background for subchannel $j$. We obtain the cross section by minimizing $-\ln L\left(\sigma,\left\{N_{j}^{o b s}, N_{j}^{b k g d}, \mathcal{B}_{j}, \mathcal{L}_{j}, A c c_{j}\right\}\right)$. The statistical uncertainty on the cross section is obtained by the usual procedure of varying the negative log-likelihood 
by a half unit above the minimum. Figure 6.1 shows the $-\ln (L)$ curve as a function of the cross section.

To determine the systematic uncertainty, a new likelihood function is derived for each independent source of systematics. The theoretical uncertainty on the signal cross section is only utilized here for the misreconstruction background systematic. The variations in the central value of the cross section are then summed in quadrature to obtain the total systematic uncertainty.

The measured physical cross section of $Z / \gamma^{*} Z / \gamma^{*}$ production in the mass region $M_{1}\left(Z / \gamma^{*}\right)>$ $30 \mathrm{GeV}, M_{2}\left(Z / \gamma^{*}\right)>30$ is found to be:

$$
\sigma\left(p \bar{p} \rightarrow Z / \gamma^{*} Z / \gamma^{*}\right)=1.26_{-0.36}^{+0.44}(\text { stat })_{-0.15}^{+0.17} \text { (syst) } \pm 0.08 \text { (lumi) pb. }
$$

We then calculate the ratio of $\sigma\left(p \bar{p} \rightarrow Z / \gamma^{*} Z / \gamma^{*}\right)$ to $\sigma(p \bar{p} \rightarrow Z Z)$ for this mass region using MCFM [44], and from this correction determine the $p \bar{p} \rightarrow Z Z$ cross section to be

$$
1.05_{-0.30}^{+0.37}(\text { stat })_{-0.12}^{+0.14} \text { (syst) } \pm 0.06 \text { (lumi) pb. }
$$

We combine this measurement with the $p \bar{p} \rightarrow Z Z$ cross section measured in the $\ell^{+} \ell^{-} \nu \bar{\nu}$ final state using data from the D0 detector [42], giving a total combined $p \bar{p} \rightarrow Z Z$ cross section of

$$
1.32_{-0.25}^{+0.29} \text { (stat) } \pm 0.12 \text { (syst) } \pm 0.04 \text { (lumi) pb. }
$$

The measured $Z Z$ cross section values are consistent with the SM expectation of $1.43 \pm 0.10$ $\mathrm{pb}[44]$.

In Fig. 6.2 we show the distributions for data, expected Higgs boson signal and background for the $p_{T}$ of the four leptons. In Fig. 6.3 are the $\Delta R$ and $\Delta \phi$ between the two leptons in each $Z$, while Fig. 6.4 gives the overall missing $E_{T}$, the $p_{T}$ of the $Z Z$ system, the dilepton and four lepton invariant masses. One can see the excellent agreement between data and the MC expectation. 


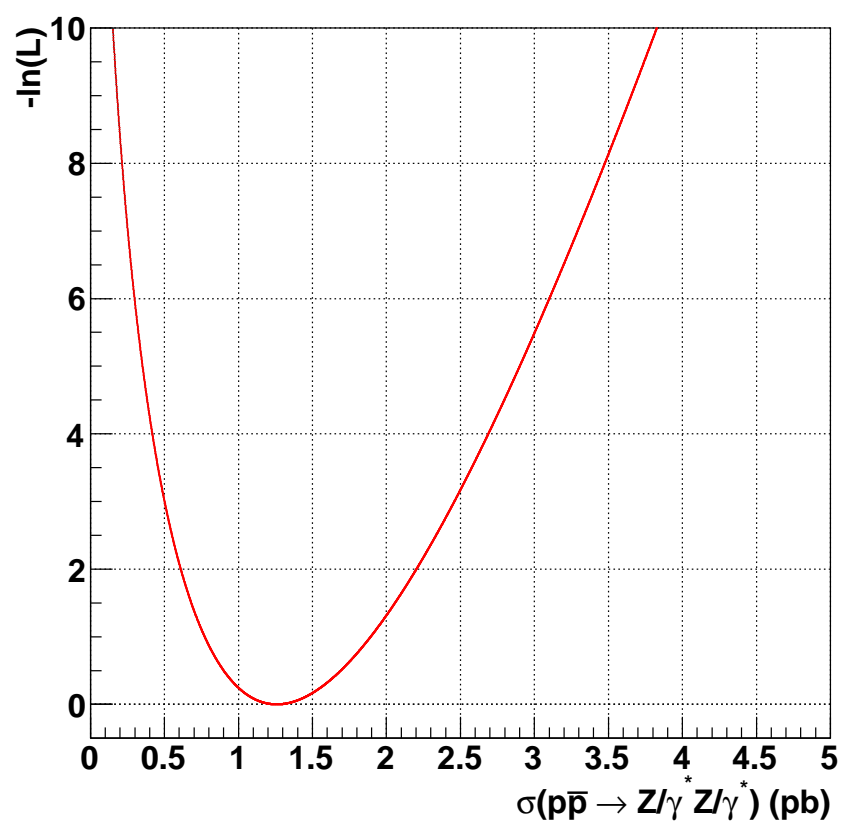

Figure 6.1: $-\ln (L)$ versus cross section (in pb). 

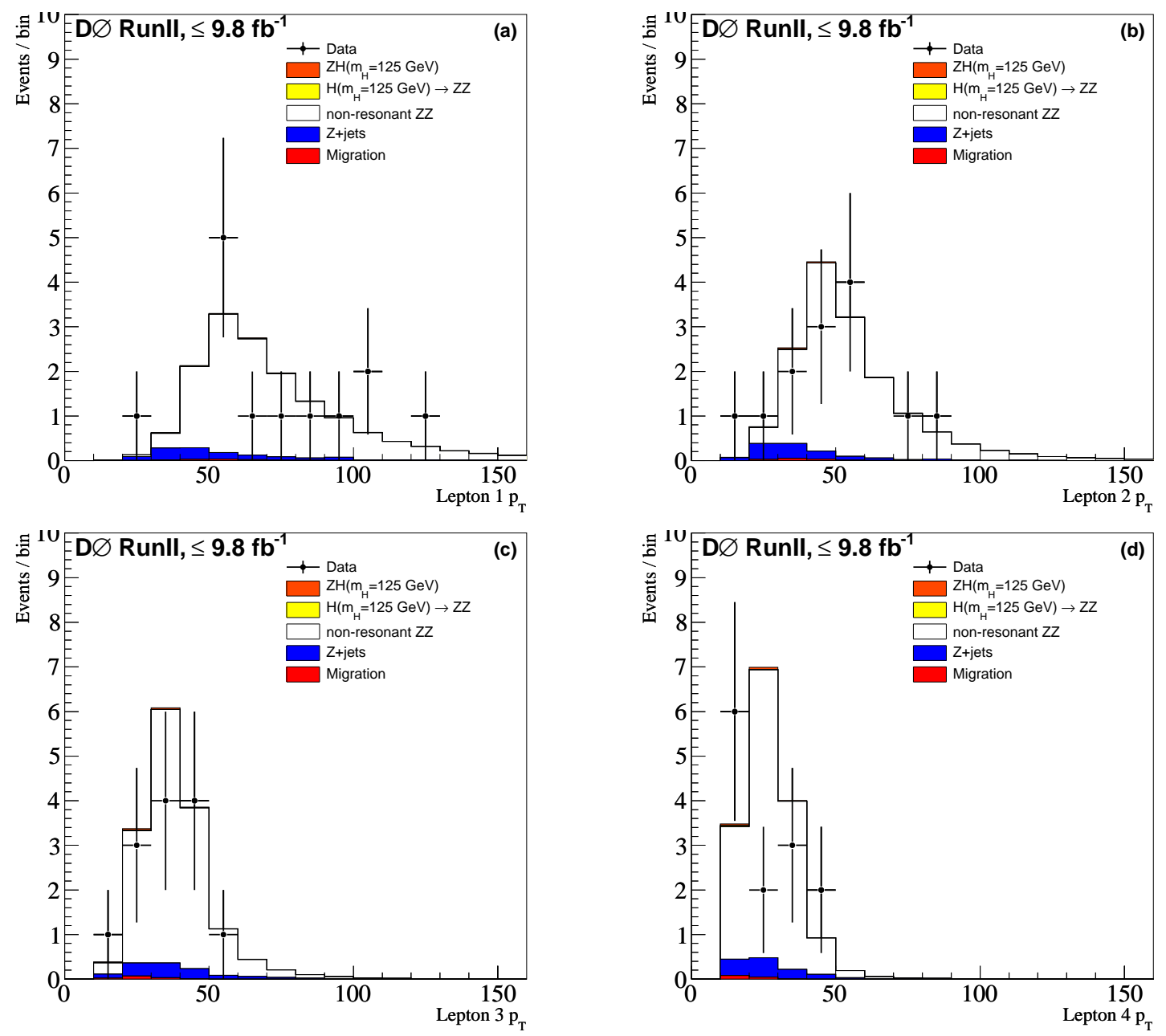

Figure 6.2: Distributions of lepton $p_{T}$ ordered in $p_{T}$ in data, expected Higgs signal and backgrounds superimposed for the combined channels. 

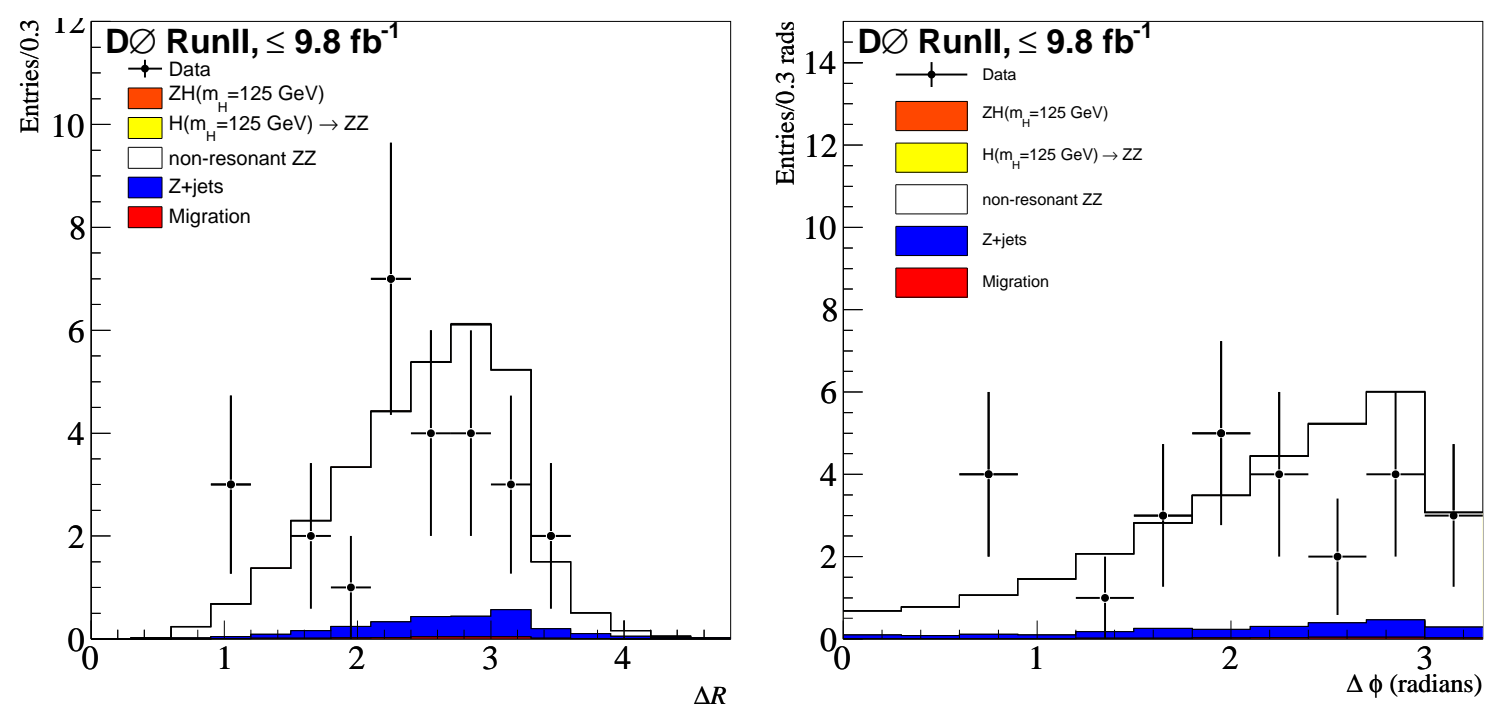

Figure 6.3: Distributions of $\Delta R$ and $\Delta \phi$ between the two leptons in each $Z$ decay in data, expected Higgs signal and backgrounds superimposed for the combined channels. 

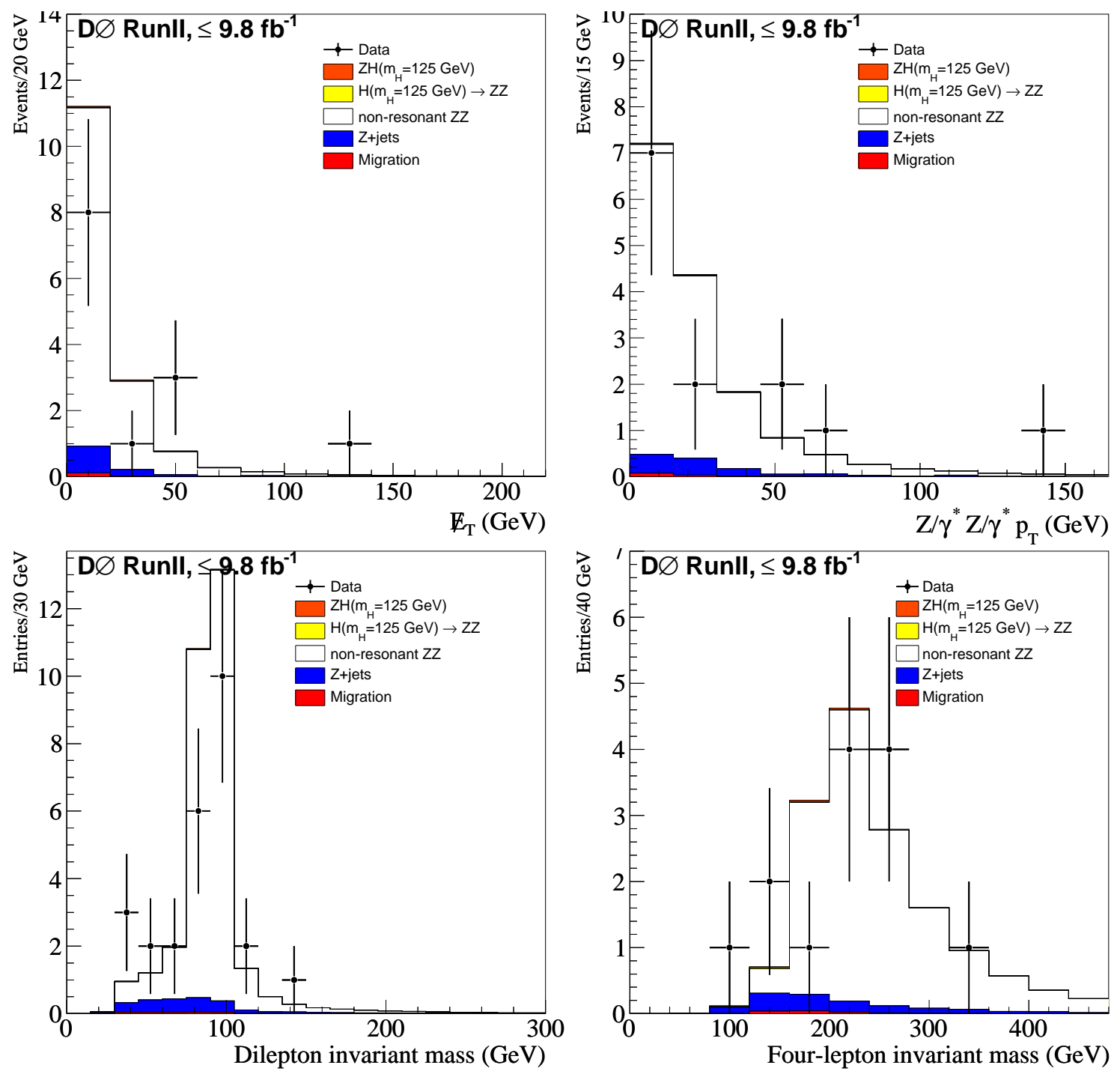

Figure 6.4: Distributions of missing transverse energy,the $p_{T}$ of the $Z Z$ system, dilepton invariant mass and four lepton invariant mass in data, expected Higgs signal and backgrounds superimposed for the combined channels. 


\section{CHAPTER 7 SYSTEMATICS}

Numerous factors contribute to systematic uncertainties in this measurement. This section describes these various factors and estimates their uncertainty. Most systematics apply to both the $Z Z$ cross section measurement and the Higgs boson search.

The considered systematics include:

- Data Quality and Trigger: The systematic uncertainty on the measured data quality flag efficiency was estimated to be $0.5 \%$ [41] by comparing the effect of data quality removal with calorimeter quality flag events removed compared to that without calorimeter quality flag events removed. We also include a $1.0 \%$ uncertainty here due to possible trigger uncertainty.

- $z_{v t x}$ Reweighting: The nominal reweighting used according to the procedure described in note [24] uses a fit from $\pm 60 \mathrm{~cm}$ in the data. An alternate fit from $\pm 40 \mathrm{~cm}$ is also available, and this is used to estimate the uncertainty. This procedure uses the shapes of the $p$ and $\bar{p}$ bunches and the $\beta_{x}^{*}$ and the $\beta_{y}^{*}$ of the interaction point for various instantaneous luminosities and $z_{v t x}$ cuts.

- $Z Z p_{T}$ Reweighting: To estimate the effect of higher order corrections on signal acceptance, we apply a $Z Z p_{T}$ reweighting function, derived by fitting the $p_{T}$ spectra 
of $W W$ in Sherpa and Pythia. We estimate this uncertainty by switching on this correction and measuring its effect on the relevant acceptance times efficiencies.

- Electron ID: The systematic errors on Electron ID have been calculated by the EMID group and their results are presented in references [29] and [25]. They include dependence on the distance to the closest jet, jet multiplicity, fits to efficiency curves and sensitivity to cuts on $p_{T}$ and calorimeter fiducial region. Since these issues are in common with our analysis, we use their results and calculate a systematic uncertainty for "Point0" electrons. For electrons between 15 and $20 \mathrm{GeV}$, the uncertainties are $6.7 \%, 4.2 \%, 3.5 \%$, and $2.9 \%$ for Run IIa, RunIIb1, RunIIb2, and RunIIb3 respectively (the same uncertainty is used in RunIIb3 and RunIIb4), for a luminosity weighted average of $3.7 \%$. The systematic uncertainty is smaller at higher $p_{T}$, but we use this number to be conservative. This gives us an overall uncertainty of $14.8 \%$ on the $4 e$ channel, and $7.4 \%$ on the $2 e 2 \mu$ channel. We use a systematic uncertainty of $6 \%$ per ICR electron, following [32, 33], leading to a systematic uncertainty of $12.6 \%$ on $4 e$ events with an ICR electron.

- Electron energy resolution: We apply additional smearing to the energies of the electrons to reproduce data resolution. We estimate this uncertainty by switching off this correction and measuring its effect on the relevant acceptance times effeciencies.

- Electron Fakerate: The systematic error for the electron QCD fakerate is determined by using a second form of the fakerate where the energy from the electron associated with the probe jet is used rather than the energy from the probe jet itself. The QCD background is recalculated to estimate the uncertainty.

- Muon ID: The systematic errors on Muon ID have been calculated by the Muon ID group $[26,35]$. The RunIIa systematic uncertainty for loose muons $(0.5 \%)$ is 
used here. This includes tag and probe bias, background and cut variations and finite binning uncertainties which are in common with our analysis, hence our use of their results. The RunIIb systematic uncertainty for loose muons is found to be $0.9 \%$ per muon. nseg $=0$ muons are not part of the muon certification and are not as well modeled in $\mathrm{MC}$ relative to the other muon types (they have a higher efficiency in $\mathrm{MC}$ ). But, as seen above in Table 5.15 , nseg $=0$ muon are about $5 \%$ of the $\mathrm{MC}$ muon acceptance. If we assign a $10 \%$ error to this, and add this in quadrature to the systematic uncertainty of other muon topologies, we obtain a $1 \%$ error per muon, which we use as the systematic uncertainty of for all muons in this analysis.

- Muon track: The RunIIa systematic errors on muon track reconstruction (0.6\%) are also taken from note [26] while that for IIb is $1.0 \%$ from note 6326 [35]. This includes tag and probe bias, background and cut variation, luminosity and time bias, time average, finite binning and average over $\phi$ uncertainties.

- Muon Isolation: For both the RunIIa and RunIIb channels we assign a $0.6 \%$ systematic uncertainty based on results from the Muon ID group [26] [35] for the isolation criteria used in this analysis. Note combining muon ID, tracking efficiency, and isolation one obtains an overall muon uncertainty of $1.5 \%$ with $100 \%$ correlation between muons and so the uncertainty on the $4 \mu$ channel would be $6 \%$.

- Muon momentum resolution: We apply additional smearing to the muon momentum to agree with that measured in data. We estimate this uncertainty by switching off this correction and measuring its effect on the relevant acceptance times effeciencies. 
- Muon Fakerate: The systematic error for the muon QCD fakerate is determined by varying the nominal fakerate by $\pm 20 \%$ and the QCD background is recalculated to estimate the uncertainty.

- $t \bar{t}$ cross section uncertainty: We assign $\pm 20 \%$ uncertainty to the top pair production cross-section. This covers theory uncertainty of $10 \%$ at $m_{\text {top }}=170 \mathrm{GeV}$ [40], plus cross section variation due to top mass uncertainty of $\pm 2 \mathrm{GeV}$.

- PDF: For variation in signal acceptance due to PDF uncertainty we add in quadrature PDF errors estimated in the $Z Z \rightarrow \ell \ell \nu \nu[42]$ and $Z \rightarrow e e$ cross-section analysis [43]. The combination of the two errors is done because the $Z Z \rightarrow \ell \ell \nu \nu$ analysis is normalized with respect to the inclusive $Z$ cross section. We obtain an estimate of $\pm 2.5 \%$.

- $Z Z$ cross section uncertainty: For the Higgs boson analysis we assign $7.1 \%$ uncertainty on the $\sigma(Z Z)$ as quoted in [44].

- MC Statistics: The systematic error due to limited statics in the Monte Carlo samples determined from the statistical error over the mean value.

- QCD Sample Statistics: The systematic error due to limited number of events in the normalization sample, after all cuts, used to estimate QCD background determined from the statistical error over the mean value.

An additional source of systematics in all channels not included in the tables but used in the determination of significance and cross section is the $6.1 \%$ uncertainty in the luminosity determination as provided by the luminosity group [19].

The individual uncertainties for the eeee channels are listed in Tables 7.1, 7.2, 7.3, 7.4, 7.9, 7.10, 7.11, and 7.12. The uncertainties for the $\mu \mu \mu \mu$ channel is listed in Table 7.5 


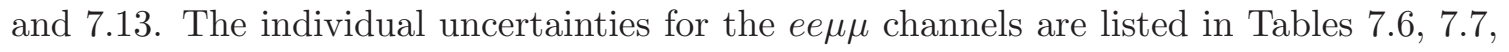
$7.8,7.14,7.15$, and 7.16 .

Table 7.1: Relative uncertainties in the RunIla eeee channel with $2 \mathrm{CC}$ electrons for various yields and backgrounds. The top nine rows are systematic uncertainties, while the next two rows are statistical in nature, and then total uncertainty is given.

\begin{tabular}{c|c|c||c|c||c|c}
\hline \hline \multirow{2}{*}{ Systematic } & \multicolumn{2}{|c||}{ signal, \% } & \multicolumn{2}{c||}{ misrec., \% } & \multicolumn{2}{c}{ QCD, \% } \\
& up & down & up & down & up & down \\
\hline Data quality & +0.50 & -0.50 & +0.50 & -0.50 & +0.50 & -0.50 \\
\hline Trigger & +1.0 & -1.0 & +1.0 & -1.0 & +1.0 & -1.0 \\
\hline$v t x_{z}$ reweight & +0.55 & -0.55 & +0.36 & -0.36 & - & - \\
\hline$Z Z p_{T}$ reweight & - & -6.04 & - & -3.18 & - & - \\
\hline Electron ID & +26.80 & -26.80 & +26.80 & -26.80 & - & - \\
\hline Electron energy res. & +1.22 & - & +1.88 & - & - & - \\
\hline EM Fakerate & - & - & - & - & +6.37 & -6.37 \\
\hline PDF & +2.50 & -2.50 & +2.50 & -2.50 & - & - \\
\hline$\sigma(Z Z)$ & +7.1 & -7.1 & +7.1 & -7.1 & - & - \\
\hline \hline MC Stats & +3.44 & -3.44 & +14.76 & -14.76 & - & - \\
\hline QCD Sample Stats & - & - & - & - & +20.06 & -20.06 \\
\hline \hline Total & +28.10 & -28.72 & +31.59 & -31.69 & +21.08 & -21.08 \\
\hline \hline
\end{tabular}


Table 7.2: Relative uncertainties in the RunIIa eeee channel with 3 CC electrons for various yields and backgrounds. The top nine rows are systematic uncertainties, while the next two rows are statistical in nature, and then total uncertainty is given.

\begin{tabular}{c||c|c||c|c||c|c}
\hline \hline \multirow{2}{*}{\multicolumn{1}{c||}{ Systematic }} & \multicolumn{2}{c||}{ signal, \% } & \multicolumn{2}{c||}{ misrec., \% } & \multicolumn{2}{c}{ QCD, \% } \\
up & down & up & down & up & down \\
\hline Data quality & +0.50 & -0.50 & +0.50 & -0.50 & +0.50 & -0.50 \\
\hline Trigger & +1.0 & -1.0 & +1.0 & -1.0 & +1.0 & -1.0 \\
\hline$v t x_{z}$ reweight & +0.53 & -0.53 & +0.62 & -0.62 & - & - \\
\hline$Z Z p_{T}$ reweight & - & -6.84 & - & -3.04 & - & - \\
\hline Electron ID & +26.80 & -26.80 & +26.80 & -26.80 & - & - \\
\hline Electron energy res. & +0.58 & - & - & -3.32 & - & - \\
\hline EM Fakerate & - & - & - & - & +4.84 & -4.84 \\
\hline PDF & +2.50 & -2.50 & +2.50 & -2.50 & - & - \\
\hline$\sigma(Z Z)$ & +7.1 & -7.1 & +7.1 & -7.1 & - & - \\
\hline \hline MC Stats & +2.26 & -2.26 & +11.17 & -11.17 & - & - \\
\hline QCD Sample Stats & - & - & - & - & +29.06 & -29.06 \\
\hline \hline Total & +27.96 & -28.78 & +30.02 & -30.36 & +29.48 & -49.48 \\
\hline \hline
\end{tabular}

Table 7.3: Relative uncertainties in the RunIIa eeee channel with 4 CC electrons for various yields and backgrounds. The top nine rows are systematic uncertainties, while the next two rows are statistical in nature, and then total uncertainty is given.

\begin{tabular}{c||c|c||c|c||c|c}
\hline \hline \multirow{2}{*}{ Systematic } & \multicolumn{2}{c||}{ signal, \% } & \multicolumn{2}{c||}{ misrec., \% } & \multicolumn{2}{c}{ QCD, \% } \\
up & down & up & down & up & down \\
\hline Data quality & +0.50 & -0.50 & +0.50 & -0.50 & +0.50 & -0.50 \\
\hline Trigger & +1.0 & -1.0 & +1.0 & -1.0 & +1.0 & -1.0 \\
\hline$v t x_{z}$ reweight & +0.57 & -0.57 & +0.53 & -0.53 & - & - \\
\hline$Z Z p_{T}$ reweight & - & -5.01 & - & -4.42 & - & - \\
\hline Electron ID & +26.80 & -26.80 & +26.80 & -26.80 & - & - \\
\hline Electron energy res. & 0.09 & - & 0.07 & - & - & - \\
\hline EM Fakerate & - & - & - & - & +9.05 & -9.05 \\
\hline PDF & +2.50 & -2.50 & +2.50 & -2.50 & - & - \\
\hline$\sigma(Z Z)$ & +7.1 & -7.1 & +7.1 & -7.1 & - & - \\
\hline \hline MC Stats & +2.27 & -2.27 & +10.79 & -10.79 & - & - \\
\hline QCD Sample Stats & - & - & - & - & +43.28 & -43.28 \\
\hline \hline Total & +27.96 & -28.40 & +28.40 & -29.88 & +44.23 & -44.23 \\
\hline \hline
\end{tabular}


Table 7.4: Relative uncertainties in the RunIIa eeee channel with 1 ICR electron for various yields and backgrounds. The top nine rows are systematic uncertainties, while the next two rows are statistical in nature, and then total uncertainty is given.

\begin{tabular}{c||c|c||c|c||c|c}
\hline \hline \multirow{2}{*}{ Systematic } & \multicolumn{2}{c||}{ signal, \% } & \multicolumn{2}{c||}{ misrec., \% } & \multicolumn{2}{c}{ QCD, \% } \\
up & down & up & down & up & down \\
\hline Data quality & +0.50 & -0.50 & +0.50 & -0.50 & +0.50 & -0.50 \\
\hline Trigger & +1.0 & -1.0 & +1.0 & -1.0 & +1.0 & -1.0 \\
\hline$v t x_{z}$ reweight & +0.59 & -0.59 & +0.57 & -0.57 & - & - \\
\hline$Z Z p_{T}$ reweight & - & -6.12 & - & -7.68 & - & - \\
\hline Electron ID & +20.97 & -20.97 & +20.97 & -20.97 & - & - \\
\hline Electron energy res. & 0.17 & - & - & -0.82 & - & - \\
\hline EM Fakerate & - & - & - & - & +0.61 & -63.23 \\
\hline PDF & +2.50 & -2.50 & +2.50 & -2.50 & - & - \\
\hline$\sigma(Z Z)$ & +7.1 & -7.1 & +7.1 & -7.1 & - & - \\
\hline \hline MC Stats & +4.06 & -4.06 & +10.49 & -10.49 & - & - \\
\hline QCD Sample Stats & - & - & - & - & +66.69 & -66.69 \\
\hline \hline Total & +22.68 & -23.49 & +24.66 & -25.84 & +66.70 & -91.91 \\
\hline \hline
\end{tabular}

Table 7.5: Relative uncertainties in the RunIIa $\mu \mu \mu \mu$ channel for various yields and backgrounds. The top eleven rows are systematic uncertainties, while the next two rows are statistical in nature, and then total uncertainty is given.

\begin{tabular}{c||c|c||c|c||c|c}
\hline \hline \multirow{2}{*}{\multicolumn{1}{c||}{ Systematic }} & \multicolumn{2}{c||}{ signal, \% } & \multicolumn{2}{c||}{ misrec., \% } & \multicolumn{2}{c}{ QCD, \% } \\
& up & down & up & down & up & down \\
\hline Data quality & +0.5 & -0.5 & +0.5 & -0.5 & +0.5 & -0.5 \\
\hline Trigger & +1.0 & -1.0 & +1.0 & -1.0 & +1.0 & -1.0 \\
\hline \hline$v t x_{z}$ reweight & +0.57 & -0.57 & +0.33 & -0.33 & - & - \\
\hline$Z Z p_{T}$ reweight & - & -0.41 & +18.28 & - & - & - \\
\hline Muon ID & +4.0 & -4.0 & +4.0 & -4.0 & - & - \\
\hline Muon momentum res. & +0.31 & - & - & -8.70 & - & - \\
\hline Muon Track & +2.4 & -2.4 & +2.4 & -2.4 & - & - \\
\hline Muon Isolation & +2.4 & -2.4 & +2.4 & -2.4 & - & - \\
\hline Muon Fakerate & - & - & - & - & +44.00 & -36.00 \\
\hline PDF & +2.5 & -2.5 & +2.5 & -2.5 & - & - \\
\hline$\sigma(Z Z)$ & +7.1 & -7.1 & +7.1 & -7.1 & - & - \\
\hline \hline MC Stats & +0.94 & -0.94 & +8.79 & -8.79 & - & - \\
\hline QCD Sample Stats & - & - & - & - & +4.50 & -4.50 \\
\hline \hline Total & +9.31 & -9.32 & +22.29 & -15.44 & +44.24 & -36.30 \\
\hline \hline
\end{tabular}




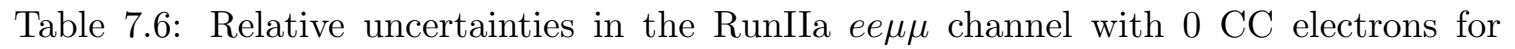
various yields and backgrounds. The top fourteen rows are systematic uncertainties, while the next two rows are statistical in nature, and then total uncertainty is given.

\begin{tabular}{|c|c|c|c|c|c|c|c|c|}
\hline \multirow[b]{2}{*}{ Systematic } & \multicolumn{2}{|c|}{ signal, $\%$} & \multicolumn{2}{|c|}{ misrec., $\%$} & \multicolumn{2}{|c|}{$t \bar{t}, \%$} & \multicolumn{2}{|c|}{$\mathrm{QCD}, \%$} \\
\hline & up & down & up & down & up & down & up & down \\
\hline Data quality & +0.5 & -0.5 & +0.5 & -0.5 & +0.5 & -0.5 & +0.5 & -0.5 \\
\hline Trigger & +1.0 & -1.0 & +1.0 & -1.0 & +1.0 & -1.0 & +1.0 & -1.0 \\
\hline$v t x_{z}$ reweight & +0.09 & -0.09 & +1.46 & -1.46 & +100.00 & -100.00 & - & - \\
\hline$Z Z p_{T}$ reweight & - & -0.03 & - & -32.65 & - & - & - & - \\
\hline Electron ID & +7.40 & -7.40 & +7.40 & -7.40 & +7.40 & -7.40 & - & - \\
\hline EM Fakerate & - & - & - & - & - & - & +1.95 & -1.95 \\
\hline Muon ID & +2.00 & -2.00 & +2.00 & -2.00 & +2.00 & -2.00 & - & - \\
\hline Muon Track & +1.20 & -1.20 & +1.20 & -1.20 & +1.20 & -1.20 & - & - \\
\hline Muon Isolation & +1.20 & -1.20 & +1.20 & -1.20 & +1.20 & -1.20 & - & - \\
\hline Muon Fakerate & - & - & - & - & - & - & +9.94 & -8.13 \\
\hline Lepton momentum res. & +0.53 & - & +0.00 & - & +100.00 & -100.00 & - & - \\
\hline$\sigma(t \bar{t})$ & - & - & - & - & +20.00 & -20.00 & - & - \\
\hline $\mathrm{PDF}$ & +2.5 & -2.5 & +2.5 & -2.5 & - & - & - & - \\
\hline$\sigma(Z Z)$ & +7.1 & -7.1 & +7.1 & -7.1 & - & - & - & - \\
\hline MC Stats & +4.71 & -4.71 & +135.76 & -69.82 & +100.0 & -100.0 & - & - \\
\hline QCD Sample Stats & - & - & - & - & - & - & +75.25 & -47.30 \\
\hline Total & +11.92 & -11.90 & +136.21 & -77.86 & +174.55 & -174.55 & +75.94 & -48.05 \\
\hline
\end{tabular}


Table 7.7: Relative uncertainties in the RunIIa ee $\mu \mu$ channel with 1 CC electrons for various yields and backgrounds. The top fourteen rows are systematic uncertainties, while the next two rows are statistical in nature, and then total uncertainty is given.

\begin{tabular}{|c|c|c|c|c|c|c|c|c|}
\hline \multirow[b]{2}{*}{ Systematic } & \multicolumn{2}{|c|}{ signal, $\%$} & \multicolumn{2}{|c|}{ misrec., $\%$} & \multicolumn{2}{|c|}{$t \bar{t}, \%$} & \multicolumn{2}{|c|}{ QCD, $\%$} \\
\hline & up & down & up & down & up & down & up & down \\
\hline Data quality & +0.5 & -0.5 & +0.5 & -0.5 & +0.5 & -0.5 & +0.5 & -0.5 \\
\hline Trigger & +1.0 & -1.0 & +1.0 & -1.0 & +1.0 & -1.0 & +1.0 & -1.0 \\
\hline$v t x_{z}$ reweight & +0.44 & -0.44 & +0.57 & -0.57 & +1.35 & -1.35 & - & - \\
\hline$Z Z p_{T}$ reweight & +0.65 & - & +9.40 & - & -27.91 & - & - & - \\
\hline Electron ID & +7.40 & -7.40 & +7.40 & -7.40 & +7.40 & -7.40 & - & - \\
\hline EM Fakerate & - & - & - & - & - & - & +6.02 & -6.02 \\
\hline Muon ID & +2.00 & -2.00 & +2.00 & -2.00 & +2.00 & -2.00 & - & - \\
\hline Muon Track & +1.2 & -1.2 & +1.2 & -1.2 & +1.2 & -1.2 & - & - \\
\hline Muon Isolation & +1.2 & -1.2 & +1.2 & -1.2 & +1.2 & -1.2 & - & - \\
\hline Muon Fakerate & - & - & - & - & - & - & +8.65 & -3.74 \\
\hline Lepton momentum res. & +0.97 & - & - & -15.99 & +3.05 & -3.05 & - & - \\
\hline$\sigma(t \bar{t})$ & - & - & - & - & +20.00 & -20.00 & - & - \\
\hline $\mathrm{PDF}$ & +2.5 & -2.5 & +2.5 & -2.5 & - & - & - & - \\
\hline$\sigma(Z Z)$ & +7.1 & -7.1 & +7.1 & -7.1 & - & - & - & - \\
\hline MC Stats & +2.01 & -2.01 & +51.62 & -38.32 & +135.76 & -69.82 & - & - \\
\hline QCD Sample Stats & - & - & - & - & - & - & +24.68 & -21.21 \\
\hline Total & +11.19 & -11.13 & +52.77 & -51.21 & +137.52 & -73.18 & +25.56 & -22.17 \\
\hline
\end{tabular}


Table 7.8: Relative uncertainties in the RunIIa eе $\mu \mu$ channel with $2 \mathrm{CC}$ electrons for various yields and backgrounds. The top fourteen rows are systematic uncertainties, while the next two rows are statistical in nature, and then total uncertainty is given.

\begin{tabular}{|c|c|c|c|c|c|c|c|c|}
\hline \multirow[b]{2}{*}{ Systematic } & \multicolumn{2}{|c|}{ signal, $\%$} & \multicolumn{2}{|c|}{ misrec., $\%$} & \multicolumn{2}{|c|}{$t \bar{t}, \%$} & \multicolumn{2}{|c|}{ QCD, \% } \\
\hline & up & down & up & down & up & down & up & down \\
\hline Data quality & +0.5 & -0.5 & +0.5 & -0.5 & +0.5 & -0.5 & +0.5 & -0.5 \\
\hline Trigger & +1.0 & -1.0 & +1.0 & -1.0 & +1.0 & -1.0 & +1.0 & -1.0 \\
\hline$v t x_{z}$ reweight & +0.31 & -0.31 & +2.08 & -2.08 & +0.75 & -0.75 & - & - \\
\hline$Z Z p_{T}$ reweight & +0.82 & - & - & -11.53 & - & - & - & - \\
\hline Electron ID & +7.40 & -7.40 & +7.40 & -7.40 & +7.40 & -7.40 & - & - \\
\hline EM Fakerate & - & - & - & - & - & - & +1.95 & -1.95 \\
\hline Muon ID & +2.00 & -2.00 & +2.00 & -2.00 & +2.00 & -2.00 & - & - \\
\hline Muon Track & +1.2 & -1.2 & +1.2 & -1.2 & +1.2 & -1.2 & - & - \\
\hline Muon Isolation & +1.2 & -1.2 & +1.2 & -1.2 & +1.2 & -1.2 & - & - \\
\hline Muon Fakerate & - & - & - & - & - & - & +2.92 & -2.43 \\
\hline Lepton momentum res. & +1.01 & - & - & -32.87 & +49.43 & -49.43 & - & - \\
\hline$\sigma(t \bar{t})$ & - & - & - & - & +20.00 & -20.00 & - & - \\
\hline $\mathrm{PDF}$ & +2.5 & -2.5 & +2.5 & -2.5 & - & - & - & - \\
\hline$\sigma(Z Z)$ & +7.1 & -7.1 & +7.1 & -7.1 & - & - & - & - \\
\hline MC Stats & +1.43 & -1.43 & +128.77 & -76.11 & +135.76 & $\begin{array}{l}-69.81 \\
\end{array}$ & - & - \\
\hline QCD Sample Stats & - & - & - & - & - & - & +25.77 & -22.09 \\
\hline Total & +11.11 & -11.03 & +129.25 & -84.44 & +129.25 & -84.44 & +26.03 & -22.34 \\
\hline
\end{tabular}


Table 7.9: Relative uncertainties in the RunIIb eeee channel with $2 \mathrm{CC}$ electrons for various yields and backgrounds. The top nine rows are systematic uncertainties, while the next two rows are statistical in nature, and then total uncertainty is given.

\begin{tabular}{c||c|c||c|c||c|c}
\hline \hline \multirow{2}{*}{\multicolumn{1}{c||}{ Systematic }} & \multicolumn{2}{c||}{ signal, \% } & \multicolumn{2}{c||}{ misrec., \% } & \multicolumn{2}{c}{ QCD, \% } \\
up & down & up & down & up & down \\
\hline Data quality & +0.5 & -0.5 & +0.5 & -0.5 & +0.5 & -0.5 \\
\hline Trigger & +1.0 & -1.0 & +1.0 & -1.0 & +1.0 & -1.0 \\
\hline$v t x_{z}$ reweight & +0.41 & -0.41 & +0.44 & -0.44 & - & - \\
\hline$Z Z p_{T}$ reweight & - & -5.11 & - & -0.56 & - & - \\
\hline Electron ID & +13.20 & -13.20 & +13.20 & -13.20 & - & - \\
\hline Electron energy res. & +0.96 & - & - & -0.26 & - & - \\
\hline EM Fakerate & - & - & - & - & +21.25 & -21.25 \\
\hline PDF & +2.5 & -2.5 & +2.5 & -2.5 & - & - \\
\hline$\sigma(Z Z)$ & +7.1 & -7.1 & +7.1 & -7.1 & - & - \\
\hline \hline MC Stats & +2.47 & -2.47 & +6.45 & -6.45 & - & - \\
\hline QCD Sample Stats & - & - & - & - & +8.59 & -8.59 \\
\hline \hline Total & +15.47 & -16.26 & +16.55 & -16.56 & +22.95 & -22.95 \\
\hline \hline
\end{tabular}


Table 7.10: Relative uncertainties in the RunIIb eeee channel with $3 \mathrm{CC}$ electrons for various yields and backgrounds. The top nine rows are systematic uncertainties, while the next two rows are statistical in nature, and then total uncertainty is given.

\begin{tabular}{c||c|c||c|c||c|c}
\hline \multicolumn{1}{c||}{} & \multicolumn{2}{c||}{ signal, $\%$} & \multicolumn{2}{c||}{ misrec., \% } & \multicolumn{2}{c}{ QCD, \% } \\
Systematic & up & down & up & down & up & down \\
\hline Data quality & +0.5 & -0.5 & +0.5 & -0.5 & +0.5 & -0.5 \\
\hline Trigger & +1.0 & -1.0 & +1.0 & -1.0 & +1.0 & -1.0 \\
\hline$v t x_{z}$ reweight & +0.43 & -0.43 & +0.45 & -0.45 & - & - \\
\hline$Z Z p_{T}$ reweight & - & -5.44 & - & -4.65 & - & - \\
\hline Electron ID & +13.20 & -13.20 & +13.20 & -13.20 & - & - \\
\hline Electron energy res. & +0.72 & - & - & +1.25 & - & - \\
\hline EM Fakerate & - & - & - & - & +16.96 & -16.96 \\
\hline PDF & +2.5 & -2.5 & +2.5 & -2.5 & - & - \\
\hline$\sigma(Z Z)$ & +7.1 & -7.1 & +7.1 & -7.1 & - & - \\
\hline \hline MC Stats & +2.53 & -2.53 & +5.08 & -5.08 & - & - \\
\hline QCD Sample Stats & - & - & - & - & +9.51 & -9.51 \\
\hline \hline Total & +15.47 & -16.38 & +16.12 & -16.73 & +19.48 & -19.48 \\
\hline \hline
\end{tabular}

Table 7.11: Relative uncertainties in the RunIIb eeee channel with $4 \mathrm{CC}$ electrons for various yields and backgrounds. The top nine rows are systematic uncertainties, while the next two rows are statistical in nature, and then total uncertainty is given.

\begin{tabular}{c||c|c||c|c||c|c}
\hline \hline \multirow{2}{*}{ Systematic } & \multicolumn{2}{c||}{ signal, \% } & \multicolumn{2}{c||}{ misrec., \% } & \multicolumn{2}{c}{ QCD, \% } \\
& up & down & up & down & up & down \\
\hline Data quality & +0.5 & -0.5 & +0.5 & -0.5 & +0.5 & -0.5 \\
\hline Trigger & +1.0 & -1.0 & +1.0 & -1.0 & +1.0 & -1.0 \\
\hline \hline$v t x_{z}$ reweight & +0.46 & -0.46 & +0.36 & -0.36 & - & - \\
\hline$Z Z p_{T}$ reweight & - & -5.18 & - & -5.72 & - & - \\
\hline Electron ID & +13.20 & -13.20 & +13.20 & -13.20 & - & - \\
\hline Electron energy res. & +0.35 & - & +0.30 & - & - & - \\
\hline EM Fakerate & - & - & - & - & +10.49 & -10.49 \\
\hline PDF & +2.5 & -2.5 & +2.5 & -2.5 & - & - \\
\hline$\sigma(Z Z)$ & +7.1 & -7.1 & +7.1 & -7.1 & - & - \\
\hline \hline MC Stats & +3.08 & -3.08 & +4.87 & -4.87 & - & - \\
\hline QCD Sample Stats & - & - & - & - & +11.96 & -11.96 \\
\hline \hline Total & +15.56 & -16.39 & +16.00 & -16.99 & +15.95 & -15.95 \\
\hline \hline
\end{tabular}


Table 7.12: Relative uncertainties in the RunIIb eeee channel with 1 ICR electron for various yields and backgrounds. The top nine rows are systematic uncertainties, while the next two rows are statistical in nature, and then total uncertainty is given.

\begin{tabular}{c||c|c||c|c||c|c}
\hline \hline \multirow{2}{*}{\multicolumn{1}{c||}{ Systematic }} & \multicolumn{2}{c||}{ signal, \% } & \multicolumn{2}{c||}{ misrec., \% } & \multicolumn{2}{c}{ QCD, \% } \\
Data quality & +0.5 & -0.5 & +0.5 & -0.5 & +0.5 & -0.5 \\
\hline Trigger & +1.0 & -1.0 & +1.0 & -1.0 & +1.0 & -1.0 \\
\hline \hline$v t x_{z}$ reweight & +0.46 & -0.46 & +0.46 & -0.46 & - & - \\
\hline$Z Z p_{T}$ reweight & - & -5.08 & - & -4.94 & - & - \\
\hline Electron ID & +11.58 & -11.58 & +11.58 & -11.58 & - & - \\
\hline Electron energy res. & +0.58 & - & - & -0.75 & - & - \\
\hline EM Fakerate & - & - & - & - & +0.17 & -40.71 \\
\hline PDF & +2.5 & -2.5 & +2.5 & -2.5 & - & - \\
\hline$\sigma(Z Z)$ & +7.1 & -7.1 & +7.1 & -7.1 & - & - \\
\hline \hline MC Stats & +2.35 & -2.35 & +4.94 & -4.94 & - & - \\
\hline QCD Sample Stats & - & - & - & - & +14.02 & -14.02 \\
\hline \hline Total & +14.07 & -14.95 & +14.72 & -15.54 & +14.07 & -43.07 \\
\hline \hline
\end{tabular}

Table 7.13: Relative uncertainties in the RunIIb $\mu \mu \mu \mu$ channel for various yields and backgrounds. The top eleven rows are systematic uncertainties, while the next two rows are statistical in nature, and then total uncertainty is given.

\begin{tabular}{c||c|c||c|c||c|c}
\hline \hline \multicolumn{1}{c||}{} & \multicolumn{2}{c||}{ signal, \% } & \multicolumn{2}{c||}{ misrec., \% } & \multicolumn{2}{c}{ QCD, \% } \\
Systematic & up & down & up & down & up & down \\
\hline Data quality & +0.5 & -0.5 & +0.5 & -0.5 & +0.5 & -0.5 \\
\hline Trigger & +1.0 & -1.0 & +1.0 & -1.0 & +1.0 & -1.0 \\
\hline \hline$v t x_{z}$ reweight & +0.32 & -0.32 & +0.41 & -0.41 & - & - \\
\hline$Z Z p_{T}$ reweight & +0.20 & - & +17.44 & - & - & - \\
\hline Muon ID & +4.0 & -4.0 & +4.0 & -4.0 & - & - \\
\hline Muon momentum res. & +0.32 & - & - & -2.85 & - & - \\
\hline Muon Track & +4.0 & -4.0 & +4.0 & -4.0 & - & - \\
\hline Muon Isolation & +2.4 & -2.4 & +2.4 & -2.4 & - & - \\
\hline Muon Fakerate & - & - & - & - & +44.34 & -36.62 \\
\hline PDF & +2.5 & -2.5 & +2.5 & -2.5 & - & - \\
\hline$\sigma(Z Z)$ & +7.1 & -7.1 & +7.1 & -7.1 & - & - \\
\hline \hline MC Stats & +0.45 & -0.45 & +4.14 & -4.14 & - & - \\
\hline QCD Sample Stats & - & - & - & - & +1.34 & -1.34 \\
\hline \hline Total & +9.80 & -9.80 & +20.42 & -11.01 & +44.37 & -36.66 \\
\hline \hline
\end{tabular}




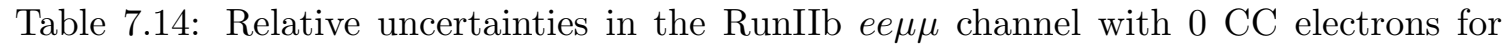
various yields and backgrounds. The top fourteen rows are systematic uncertainties, while the next two rows are statistical in nature, and then total uncertainty is given.

\begin{tabular}{|c|c|c|c|c|c|c|c|c|}
\hline \multirow[b]{2}{*}{ Systematic } & \multicolumn{2}{|c|}{ signal, $\%$} & \multicolumn{2}{|c|}{ misrec., $\%$} & \multicolumn{2}{|c|}{$t \bar{t}, \%$} & \multicolumn{2}{|c|}{$\mathrm{QCD}, \%$} \\
\hline & up & down & up & down & up & down & up & down \\
\hline Data quality & +0.5 & -0.5 & +0.5 & -0.5 & +0.5 & -0.5 & +0.5 & -0.5 \\
\hline Trigger & +1.0 & -1.0 & +1.0 & -1.0 & +1.0 & -1.0 & +1.0 & -1.0 \\
\hline$v t x_{z}$ reweight & +1.00 & -1.00 & +9.63 & -9.63 & +0.89 & -0.89 & - & - \\
\hline$Z Z p_{T}$ reweight & +2.11 & - & - & -40.84 & - & - & - & - \\
\hline Electron ID & +7.40 & -7.40 & +7.40 & -7.40 & +7.40 & -7.40 & - & - \\
\hline EM Fakerate & - & - & - & - & - & - & +10.61 & -10.61 \\
\hline Muon ID & +2.00 & -2.00 & +2.00 & -2.00 & +2.00 & -2.00 & - & - \\
\hline Muon Track & +2.00 & -2.00 & +2.00 & -2.00 & +2.00 & -2.00 & - & - \\
\hline Muon Isolation & +1.20 & -1.20 & +1.20 & -1.20 & +1.20 & -1.20 & - & - \\
\hline Muon Fakerate & - & - & - & - & - & - & +4.48 & -3.71 \\
\hline Lepton momentum res. & +1.41 & - & - & -19.61 & - & - & - & - \\
\hline$\sigma(t \bar{t})$ & - & - & - & - & +20.00 & -20.00 & - & - \\
\hline $\mathrm{PDF}$ & +2.5 & -2.5 & +2.5 & -2.5 & - & - & - & - \\
\hline$\sigma(Z Z)$ & +7.1 & -7.1 & +7.1 & -7.1 & - & - & - & - \\
\hline MC Stats & +2.32 & -2.32 & +42.70 & -33.19 & +135.75 & -69.81 & - & - \\
\hline QCD Sample Stats & - & - & - & - & - & - & +3.60 & -3.60 \\
\hline Total & +11.42 & -11.53 & +45.15 & -58.04 & +137.47 & -73.08 & +12.12 & -11.86 \\
\hline
\end{tabular}


Table 7.15: Relative uncertainties in the RunIIb ee $\mu \mu$ channel with $1 \mathrm{CC}$ electrons for various yields and backgrounds. The top fourteen rows are systematic uncertainties, while the next two rows are statistical in nature, and then total uncertainty is given.

\begin{tabular}{|c|c|c|c|c|c|c|c|c|}
\hline \multirow[b]{2}{*}{ Systematic } & \multicolumn{2}{|c|}{ signal, $\%$} & \multicolumn{2}{|c|}{ misrec., $\%$} & \multicolumn{2}{|c|}{$t \bar{t}, \%$} & \multicolumn{2}{|c|}{ QCD, $\%$} \\
\hline & up & down & up & down & up & down & up & down \\
\hline Data quality & +0.5 & -0.5 & +0.5 & -0.5 & +0.5 & -0.5 & +0.5 & -0.5 \\
\hline Trigger & +1.0 & -1.0 & +1.0 & -1.0 & +1.0 & -1.0 & +1.0 & -1.0 \\
\hline$v t x_{z}$ reweight & +2.21 & -2.21 & +1.06 & -1.06 & +0.01 & -0.01 & - & - \\
\hline$Z Z p_{T}$ reweight & +0.28 & - & - & -23.79 & - & - & - & - \\
\hline Electron ID & +7.40 & -7.40 & +7.40 & -7.40 & +7.40 & -7.40 & - & - \\
\hline EM Fakerate & - & - & - & - & - & - & +14.45 & -14.45 \\
\hline Muon ID & +2.00 & -2.00 & +2.00 & -2.00 & +2.00 & -2.00 & - & - \\
\hline Muon Track & +2.00 & -2.00 & +2.00 & -2.00 & +2.00 & -2.00 & - & - \\
\hline Muon Isolation & +1.20 & -1.20 & +1.20 & -1.20 & +1.20 & -1.20 & - & - \\
\hline Muon Fakerate & - & - & - & - & - & - & +3.17 & -2.61 \\
\hline Lepton momentum res. & - & -0.46 & - & -4.41 & - & -0.01 & - & - \\
\hline$\sigma(t \bar{t})$ & - & - & - & - & +20.00 & -20.00 & - & - \\
\hline $\mathrm{PDF}$ & +2.5 & -2.5 & +2.5 & -2.5 & - & - & - & - \\
\hline$\sigma(Z Z)$ & +7.1 & -7.1 & +7.1 & -7.1 & - & - & - & - \\
\hline MC Stats & +0.94 & -0.94 & +16.44 & -16.44 & +51.62 & -38.32 & - & - \\
\hline QCD Sample Stats & - & - & - & - & - & - & +2.33 & -2.33 \\
\hline Total & +11.31 & -11.32 & +19.84 & -31.29 & +55.95 & -43.98 & +15.02 & -14.91 \\
\hline
\end{tabular}




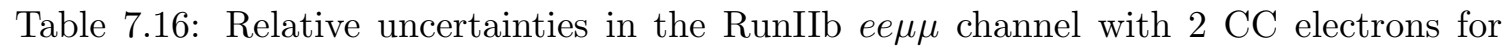
various yields and backgrounds. The top fourteen rows are systematic uncertainties, while the next two rows are statistical in nature, and then total uncertainty is given.

\begin{tabular}{|c|c|c|c|c|c|c|c|c|}
\hline \multirow[b]{2}{*}{ Systematic } & \multicolumn{2}{|c|}{ signal, $\%$} & \multicolumn{2}{|c|}{ misrec., $\%$} & \multicolumn{2}{|c|}{$t \bar{t}, \%$} & \multicolumn{2}{|c|}{$\mathrm{QCD}, \%$} \\
\hline & up & down & up & down & up & down & up & down \\
\hline Data quality & +0.5 & -0.5 & +0.5 & -0.5 & +0.5 & -0.5 & +0.5 & -0.5 \\
\hline Trigger & +1.0 & -1.0 & +1.0 & -1.0 & +1.0 & -1.0 & +1.0 & -1.0 \\
\hline$v t x_{z}$ reweight & +1.79 & -1.79 & +11.82 & -11.82 & +0.18 & -0.18 & - & - \\
\hline$Z Z p_{T}$ reweight & +1.52 & - & - & -17.43 & - & - & - & - \\
\hline Electron ID & +7.40 & -7.40 & +7.40 & -7.40 & +7.40 & -7.40 & - & - \\
\hline EM Fakerate & - & - & - & - & - & - & +12.24 & -12.24 \\
\hline Muon ID & +2.00 & -2.00 & +2.00 & -2.00 & +2.00 & -2.00 & - & - \\
\hline Muon Track & +2.00 & -2.00 & +2.00 & -2.00 & +2.00 & -2.00 & - & - \\
\hline Muon Isolation & +1.20 & -1.20 & +1.20 & -1.20 & +1.20 & -1.20 & - & - \\
\hline Muon Fakerate & - & - & - & - & - & - & +1.32 & -1.08 \\
\hline Lepton momentum res. & +1.23 & - & +11.59 & - & +99.63 & - & - & - \\
\hline$\sigma(t \bar{t})$ & - & - & - & - & +20.00 & -20.00 & - & - \\
\hline $\mathrm{PDF}$ & +2.5 & -2.5 & +2.5 & -2.5 & - & - & - & - \\
\hline$\sigma(Z Z)$ & +7.1 & -7.1 & +7.1 & -7.1 & - & - & - & - \\
\hline MC Stats & +0.70 & -0.70 & +16.67 & -16.67 & +88.27 & -55.12 & - & - \\
\hline QCD Sample Stats & - & - & - & - & - & - & +2.12 & -2.12 \\
\hline Total & +11.28 & -11.22 & +25.96 & -29.04 & +134.85 & -59.19 & +12.54 & -12.52 \\
\hline
\end{tabular}




\section{CHAPTER 8}

\section{LIMITS CALCULATION}

Evidence of the SM Higgs boson has been reported by the D0 and CDF collaborations [9] and its discovery reported by both the CMS and ATLAS collaborations $[11,10]$. We find it appropriate to perform such a search in the four lepton channel as an addition to the $Z Z$ production cross section measurement. Given we have four isolated leptons, we choose to use the 4-lepton invariant mass to search for the production of the Higgs boson.

We consider two sources of Higgs boson production: $g g \rightarrow H$ and $Z H$ production. Both are simulated using PYтніA. We are sensitive in the four-lepton final state to the production and decay mode $g g \rightarrow H \rightarrow Z Z \rightarrow \ell^{+} \ell^{-} \ell^{\prime+} \ell^{\prime-}$. The $Z H$ production mode contributes primarily through decays of $H \rightarrow \tau \tau \rightarrow \ell \nu \nu \ell \nu \nu, H \rightarrow W W \rightarrow \ell \nu \ell \nu$, and $H \rightarrow Z Z$ where at least one of the $Z$ bosons decays leptonically. All Higgs boson production cross sections and branching ratios are taken from [23].

Our signal samples were listed in Tables 3.2 through 3.5. Signal yields for Higgs boson masses points of 125 and $180 \mathrm{GeV}$ are listed in Tab. 8.1. We normalize the non-resonant $Z Z$ production to the $\mathrm{SM}$ cross section for $p \bar{p} \rightarrow Z Z \rightarrow \ell \ell \ell^{\prime} \ell^{\prime}$ with the masses of both $Z$ bosons $>30 \mathrm{GeV}$ of $8.86 \mathrm{fb}(2.21 \mathrm{fb}$ for the $\mu \mu \mu \mu$ and eeee final states each and $4.41 \mathrm{fb}$ for the $e e \mu \mu$ final state) [44].

Our systematics are presented in Chap. 7. We assess a $7 \%$ uncertainty on the nonresonant $Z Z$ cross-section. We assess a $10.9 \%$ and $6.2 \%$ systematic uncertainty on the 
Table 8.1: Signal yields for Higgs boson masses of 125 and $180 \mathrm{GeV}$. The other $H$ decays not listed individually consist of the decays $H \rightarrow \gamma \gamma, H \rightarrow \mu \mu$, and $H \rightarrow Z \gamma$.

\begin{tabular}{l|c|c|}
\hline \hline Higgs boson mass $(\mathrm{GeV})$ & 125 & 180 \\
\hline$Z H$ eeee, $4 \mathrm{CCnoICR}$ & 0.010 & 0.011 \\
$Z H$ eeee, 3CCnoICR & 0.006 & 0.006 \\
$Z H$ eeee, 2CCnoICR & 0.003 & 0.002 \\
$Z H$ eeee, 1ICR & 0.008 & 0.009 \\
$Z H$ ee $\mu$, 0CC & 0.001 & 0.001 \\
$Z H$ ee $\mu \mu, 1 \mathrm{CC}$ & 0.015 & 0.011 \\
$Z H$ ee $\mu \mu$, CC & 0.036 & 0.036 \\
$Z H \mu \mu \mu \mu$ & 0.033 & 0.031 \\
\hline$Z H, H \rightarrow W W$ & 0.041 & 0.079 \\
$Z H, H \rightarrow Z Z$ & 0.024 & 0.027 \\
$Z H, H \rightarrow \tau \tau$ & 0.044 & $<0.001$ \\
$Z H$, other $H$ decay & 0.005 & $<0.001$ \\
\hline$Z H$ total & 0.114 & 0.107 \\
\hline \hline$g g \rightarrow H \rightarrow Z Z$ eeee, 4CCnoICR & 0.004 & 0.015 \\
$g g \rightarrow H \rightarrow Z Z$ eeee, 3CCnoICR & 0.001 & 0.007 \\
$g g \rightarrow H \rightarrow Z Z$ eeee, 2CCnoICR & $<0.001$ & 0.004 \\
$g g \rightarrow H \rightarrow Z Z$ eeee, 1ICR & 0.002 & 0.012 \\
$g g \rightarrow H \rightarrow Z Z$ ee $\mu \mu$, CCC & $<0.001$ & 0.001 \\
$g g \rightarrow H \rightarrow Z Z$ ee $\mu \mu, 1 \mathrm{CC}$ & 0.002 & 0.018 \\
$g g \rightarrow H \rightarrow Z Z$ ee $\mu \mu, 2 \mathrm{CC}$ & 0.007 & 0.048 \\
$g g \rightarrow H \rightarrow Z Z \mu \mu \mu \mu$ & 0.007 & 0.046 \\
\hline$g g \rightarrow H \rightarrow Z Z$ total & 0.026 & 0.152 \\
\hline \hline Signal total & 0.137 & 0.258
\end{tabular}

predicted rates of $g g \rightarrow H$ and $Z H$ production, respectively. The electron ID, muon ID, and PDF uncertainties listed in the signal columns of Tab 7.1 through Tab 7.16, there referring to non-resonant $Z Z$, also apply to the Higgs boson samples.

We examine Higgs masses between 115 and $200 \mathrm{GeV}$ inclusive, traversing the mass region in increments of $5 \mathrm{GeV}$, for a total of 18 mass points. Limits calculation is performed using COLLIE (version V00-04-12) [45]. COLLIE uses a modified frequentist (or CLs) method $[45,47,46]$. A log-likelihood ratio (LLR) test statistic is formed using the 
Poisson probabilities for estimated background yields, the expected signal acceptance, and the number of observed events for each considered Higgs boson mass hypothesis. The confidence levels are derived by integrating the LLR distribution in pseudo-experiments using both the signal-plus-background hypothesis $\left(C L_{s+b}\right)$ and the background-only hypothesis $\left(C L_{b}\right)$. The excluded production cross section is taken to be the cross section for which the confidence level for signal, $C L_{s}=C L_{s+b} / C L_{b}$, is less than or equal to 0.05 .

Since gluon fusion processes produce a well measured final state of four isolated leptons, the four-lepton mass peak is very distinguished, which makes the four-lepton invariant mass the best variable to discriminate between this sort of signal and other backgrounds. Therefore, we use this variable to set limits on $g g \rightarrow H \rightarrow Z Z$. However, there is no such resonant peak in $Z H$ production. In the case of $Z H$, two of the leptons in the event come from the $Z$ decay. Thus, Higgs decay modes with two or more leptons will contribute to four signal signal. As most of $Z H$ signal arises from $H \rightarrow \tau^{+} \tau^{-}, H \rightarrow W W$, and $H \rightarrow Z Z$ decays, we expect large $\mathbb{E}_{T}$ in these events, due to the neutrinos from the $\tau$ and $W$ boson decays, as well as in events where one $Z$ boson from the $H \rightarrow Z Z$ decays to neutrinos. Thus, as none of our expected backgrounds has a source of real $\mathbb{E}_{T}$, this variable is used to set limits on $Z H$ production. Therefore, we use the following method of searching for the Higgs boson. For events with low $\mathbb{E}_{T}$, the four-lepton mass is used to discriminate between signal and background, while for events with large $\mathbb{E}_{T}$, the $\mathbb{E}_{T}$ is used. Examining the $\mathbb{E}_{T}$ distributions of the $g g \rightarrow H \rightarrow Z Z$ decays for a Higgs boson mass at $125 \mathrm{GeV}$, we note that for a $\mathbb{E}_{T}$ cut of $<30 \mathrm{GeV}$ most events are $g g \rightarrow H \rightarrow Z Z$, and for $>30 \mathrm{GeV}$ most are $Z H$. That is the motivation of placing a cut at $30 \mathrm{GeV}$ for all channels. The distributions of the inputs to COLLIE are shown in Fig. 8.1 for each final state separately and all combined. The four muon final state is not broken up into subchannels in COLLIE, but the four electron and two electron two muon final states are split into the same subsets 
as used in the $Z Z$ cross section calculation. The COLLIE inputs for the four eeee subsets are shown in Fig. 8.2, and the COLLIE inputs for the three ee $\mu \mu$ subsets are shown in Fig. 8.3.
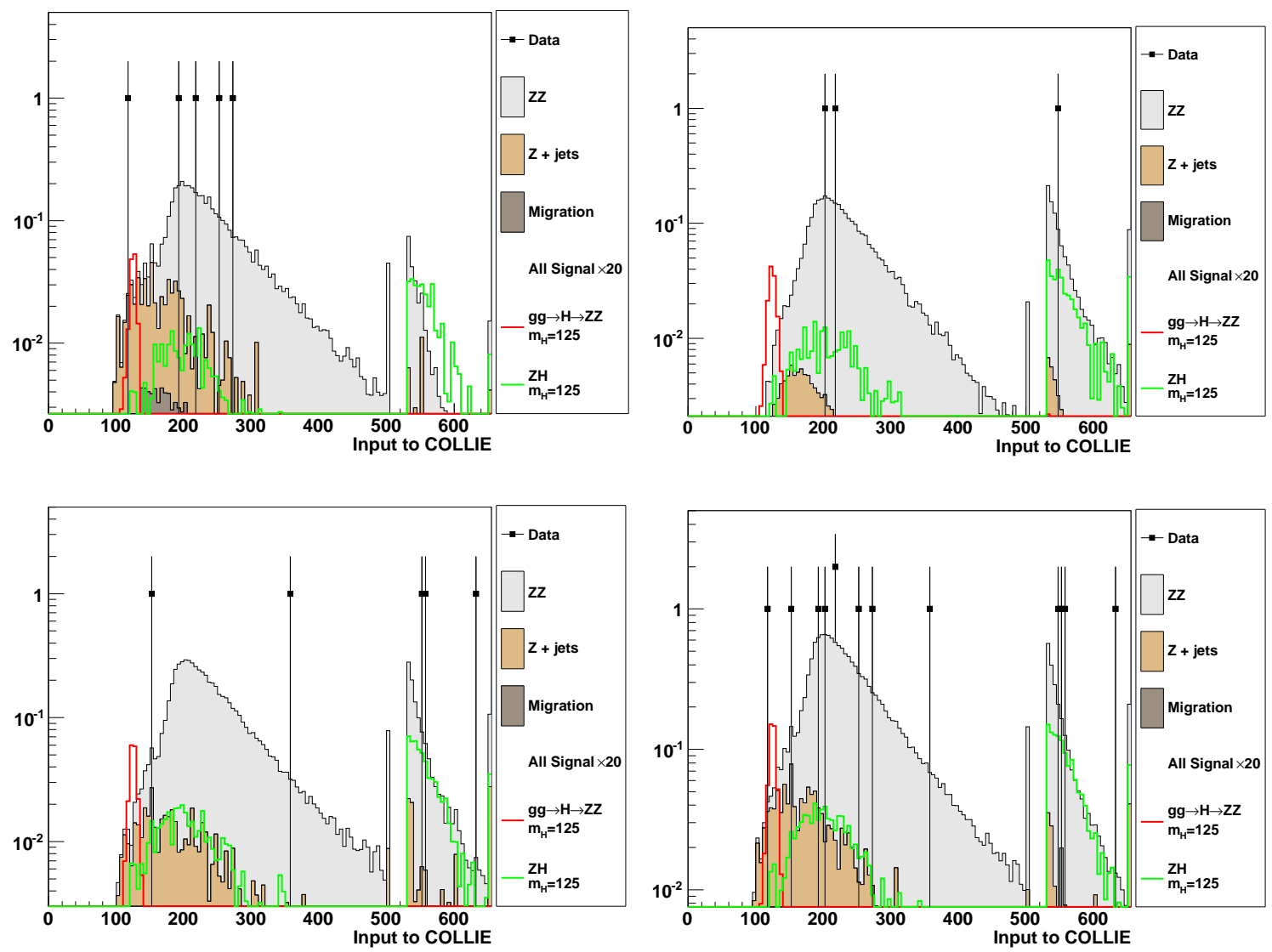

Figure 8.1: Distributions of the COLLIE inputs in the eeee (top left), $\mu \mu \mu \mu$ (top right), $e е \mu \mu$ (bottom left) and all channels combined (bottom right) for data, non-resonant $Z Z$, $Z+$ jets, migration and the Higgs boson with mass of $125 \mathrm{GeV}$. The bins between 0 and 500 are the four-lepton invariant mass between 0 and $500 \mathrm{GeV}$ with $E_{T}<30 \mathrm{GeV}$. The bins between 500 and 650 are the $\mathbb{E}_{T}$ of events with $\mathbb{E}_{T}>30 \mathrm{GeV}$; each bin corresponds to the $\not_{T}$ in $\mathrm{GeV}$ plus 500 .

The calculated limits are listed in Table 8.2. At $M_{H}=125 \mathrm{GeV}$, we expect to set a limit of 42.8 times the SM cross section at the 95\% C.L., and observe a limit of 42.3 
times the SM cross section. The limits versus $M_{H}$ are shown in Fig. 8.4, along with the associated LLR distribution. 

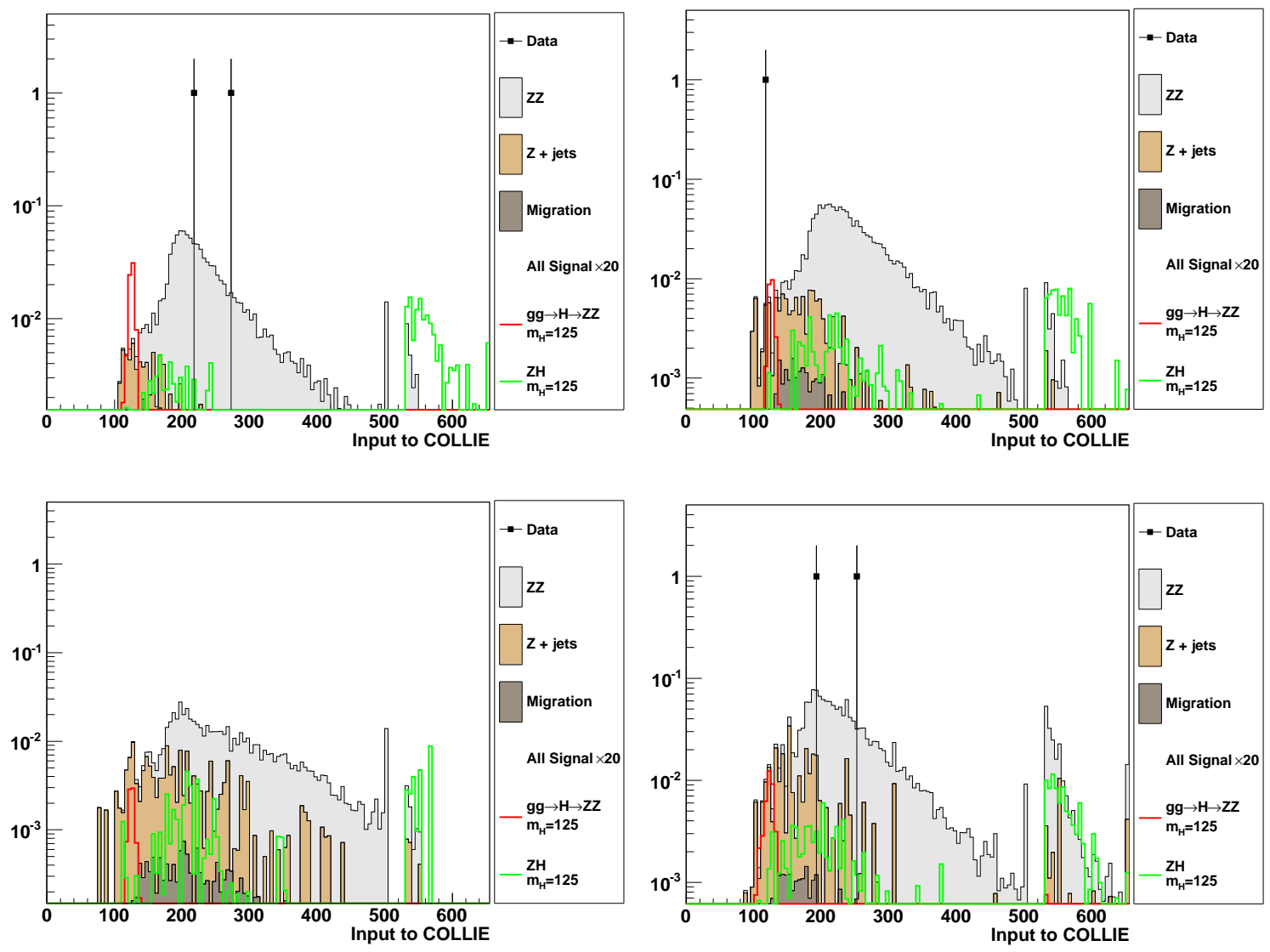

Figure 8.2: Distributions of the COLLIE inputs for each individual four electron subset for data, non-resonant $Z Z, Z+$ jets, migration and the Higgs boson with mass of $125 \mathrm{GeV}$. The $4 \mathrm{CC}$ (top left), $3 \mathrm{CC}$ (top right), and $2 \mathrm{CC}$ (bottom left) subsets have no ICR electrons. The 1 ICR(bottom right) subset has exactly 1 ICR electron and at least 2 CC electrons. The bins between 0 and 500 are the four-lepton invariant mass between 0 and $500 \mathrm{GeV}$ with $\mathbb{E}_{T}<30 \mathrm{GeV}$. The bins between 500 and 650 are the $\mathbb{E}_{T}$ of events with $\mathbb{E}_{T}>30$ $\mathrm{GeV}$; each bin corresponds to the $\mathbb{E}_{T}$ in $\mathrm{GeV}$ plus 500 . 

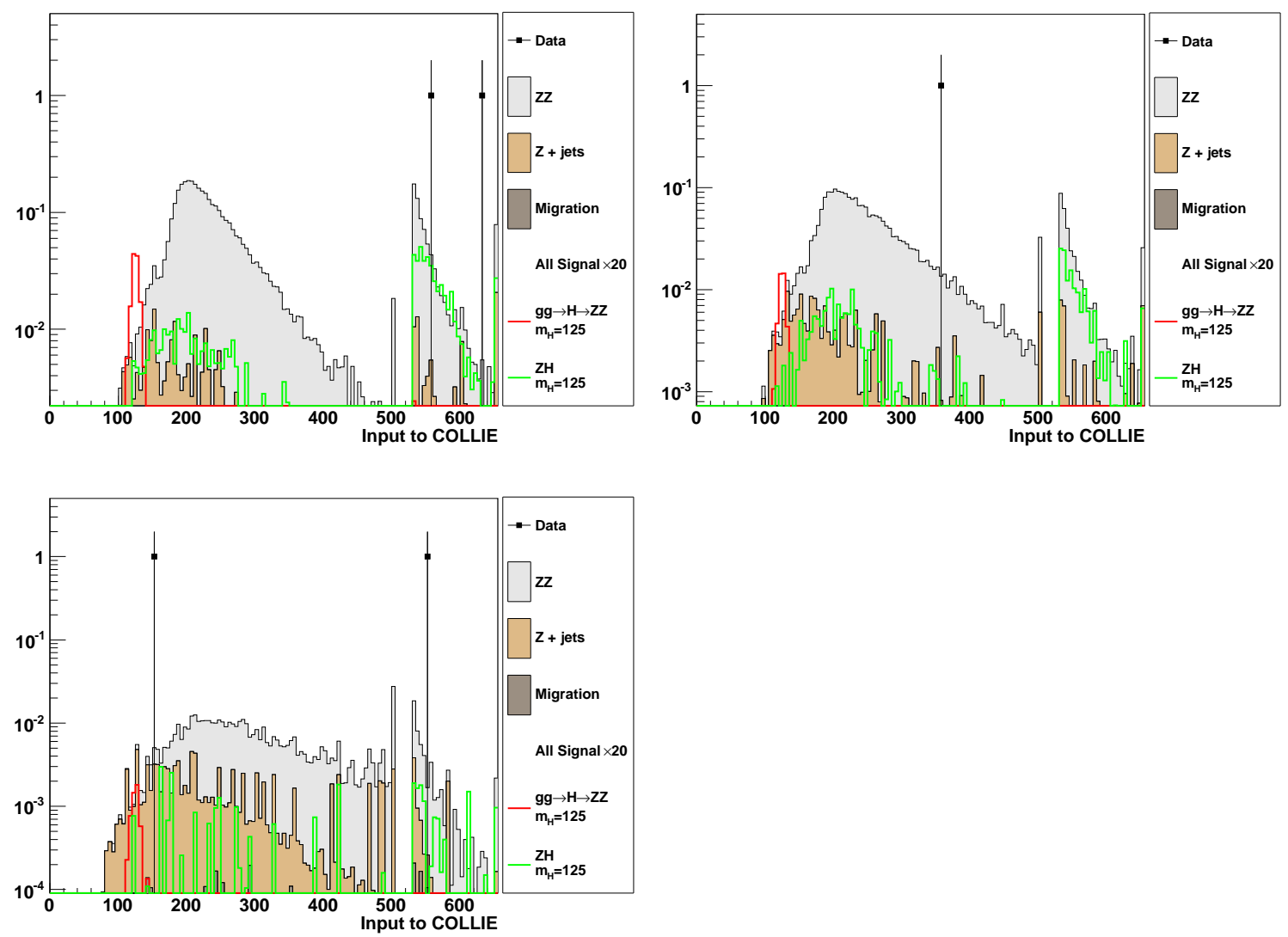

Figure 8.3: Distributions of the COLLIE inputs for each individual ee $\mu \mu$ subset for data, non-resonant $Z Z, Z+$ jets, migration and the Higgs boson with mass of $125 \mathrm{GeV}$. The 2CC (top left) subset has two CC electrons and two muons. The $1 \mathrm{CC}$ (top right) subset has one CC electron, one EC electron, and two muons. The $0 \mathrm{CC}$ subset (bottom) has two EC electrons and two muons. The bins between 0 and 500 are the four-lepton invariant mass between 0 and $500 \mathrm{GeV}$ with $\mathbb{E}_{T}<30 \mathrm{GeV}$. The bins between 500 and 650 are the $\mathbb{E}_{T}$ of events with $\mathbb{E}_{T}>30 \mathrm{GeV}$; each bin corresponds to the $\mathbb{E}_{T}$ in $\mathrm{GeV}$ plus 500. 
Table 8.2: Expected and observed 95\% C.L. upper limits on the SM Higgs boson production cross section relative to the value expected in the SM.

\begin{tabular}{c|c|c}
\hline \hline$M_{H}(\mathrm{GeV})$ & Expected & Observed \\
\hline 115 & 57.3 & 78.9 \\
120 & 54.9 & 60.6 \\
125 & 42.8 & 42.3 \\
130 & 30.6 & 33.5 \\
135 & 21.5 & 21.0 \\
140 & 16.2 & 18.2 \\
145 & 13.4 & 13.9 \\
150 & 12.4 & 12.1 \\
155 & 13.4 & 14.2 \\
160 & 20.8 & 20.6 \\
165 & 29.6 & 28.3 \\
170 & 32.3 & 39.0 \\
175 & 30.4 & 28.4 \\
180 & 22.9 & 19.6 \\
185 & 13.3 & 9.7 \\
190 & 11.8 & 8.6 \\
195 & 11.8 & 9.5 \\
200 & 12.4 & 9.9 \\
\hline \hline
\end{tabular}



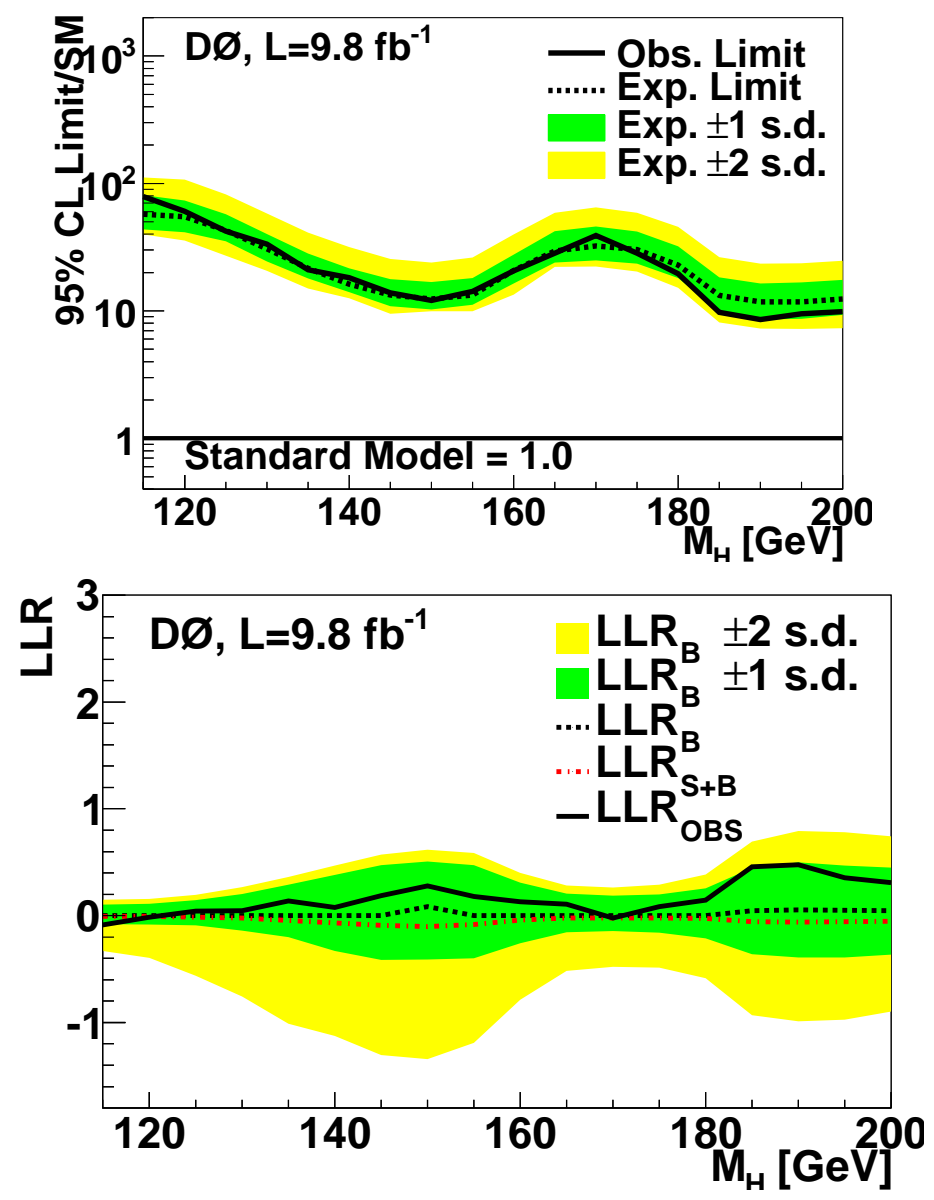

Figure 8.4: The (a) expected and observed 95\% C.L. upper limits on the SM Higgs boson production cross section relative to the value expected in the SM, and the (b) log-likelihood ratio for all four lepton channels combined. 


\section{CHAPTER 9 CONCLUSION}

With a D0 integrated luminosity up to $9.8 \mathrm{fb}^{-1}$ of RunII data we have measured the production cross section for $p \bar{p} \rightarrow Z / \gamma^{*} Z / \gamma^{*}$ with $M\left(Z / \gamma^{*}\right)>30 \mathrm{GeV}$ in the four lepton final state $(e e e e, \mu \mu \mu \mu$ and $е е \mu \mu)$ to be $1.26_{-0.36}^{+0.44}$ (stat) ${ }_{-0.15}^{+0.17}$ (syst) \pm 0.08 (lumi) pb. We correct this measurement by the expected ratio of $\sigma\left(p \bar{p} \rightarrow Z / \gamma^{*} Z / \gamma^{*}\right)$ to $\sigma(p \bar{p} \rightarrow Z Z)$

for this mass region and obtain a $p \bar{p} \rightarrow Z Z$ cross section of $1.05_{-0.30}^{+0.37}$ (stat) $)_{-0.12}^{+0.14}$ (syst) \pm 0.06 (lumi) pb.

We have also performed a search for the Higgs boson in the same final state. In this thesis we assumed that the $t$-channel $Z Z$ pair is produced with the cross section predicted by the SM. We observe no excess of data over the predicted background. Therefore, in the absence of signal we set limits on $\sigma \times B R$. At $M_{H}=125 \mathrm{GeV}$, we expect a limit of 42.8 times the SM cross section, and set a limit of 42.3 times the SM cross section at the $95 \%$ C.L. 


\section{BIBLIOGRAPHY}

[1] S.F. Novaes. Standard Model: An Introduction, arXiv:hep-ph/0001283v1.

[2] F. Halzen, A.D. Martin. Quarks and Leptons. Wiley, 1984.

[3] Standard Model Lagrangian.

URL http://home.broadpark.no/〜aklepp/astri/thesis/node52.html

[4] R. K. Ellis and W. J. Stirling and B. R. Webber. QCD and Collider Physics. Cambridge University Press, 1993.

[5] P.W. Higgs, Phys. Rev. Lett. 12,508 (1964).

[6] K. Nakamura et al. (Particle Data Group), J. Phys. G 37, 075021(2010).

[7] Wan-Ching Yang. Search for MSSM Higgs Bosons in Tau Final States with the D0 Detector. PhD Thesis, University of Manchester. 2010.

[8] R. Barate et al. (LEP Working Group for Higgs boson searches), Phys. Lett. B, 565, $61(2003)$.

[9] T. Aaltonen et al. (CDF and D0 Collaborations), Phys. Rev. D. (2013) arXiv:1303.6346.

[10] S. Chatrchyan et al. (The CMS Collaboration), Phys. Lett. B 716, 30 (2012).

[11] G. Aad et al. (The ATLAS Collaboration), Phys. Lett. B 716, 1 (2012). 
[12] V.M. Abazov et al. (The D0 Collaboration), Phys. Rev. Lett., 93, 141801 (2004).

[13] P. K. Das, Phys. Rev., D72, 055009 (2005).

[14] S. Bar-Shalom, G. Eilam and B. Mele, Phys. Lett., B500, 095008 (2001).

[15] S. Abachi, et al., Nucl. Instrum. Methods Phys. Res. A338, 185 (1994).

[16] J. Ellison, The D0 Detector Upgrade and Physics Program (2001) arXiv:0101048v2.

[17] V. M. Abazov et al. (D0 Collaboration), Nucl. Instrum. Methods, A565, 463-537 (2006).

[18] M.D. Petroff and M.G. Staplebroek, IEEE Trans. Nucl. Sci., 3 6, No. 1 (1989) 158; M.D. Petroff and M. Attac, IEEE Trans. Nucl. Sci., 36, No. 1163 (1989).

[19] "The D0 Run IIb Luminosity Measurement", B.C.K. Casey, M. Corcoran, K. DeVaughan, Y. Enari, E. Gallas, I. Katsanos, J. Linnemann, J. Orduna, R. Partridge, M. Prewitt, H. Schellman, G.R. Snow, and M. Verzocchi, Fermilab Technical Memo, FERMILAB-TM-2529-E (2012).

[20] V. M. Abazov et al. (D0 Collaboration), Nucl. Instrum. Methods, A522, 372-398 (2005).

[21] Commom Sample Group, http://www-d0.fnal.gov/Run2Physics/cs/index.html.

[22] T. Sjostrand et al., Computer Phys. Commun. 135, 238 (2001).

[23] TEVNPHWG, "Cross Section and Branching Ratio Recommendations for Tevatron Higgs Searches", CDF note 10474 (2012).

[24] H. Schellman, "The longitudinal shape of the luminous region at D0". D0 Note 5142 (2006). 
[25] https://plone4.fnal.gov/P1/D0Wiki/object-id/emid/emcert/Moriond2009/ and https://plone4.fnal.gov/P1/D0Wiki/objectid/emid/emcert/eleID_Summer2010/

[26] "Muon Identification Certification for p17", Philippe Calfayan, Thomas Gadfort, Gavin Hesketh, Vincent Lesne, Mark Owen, Raimund Stroehmer, Viatcheslav Sharyy, Boris Tuchming, D0 Note 5157

[27] O. Brandt, et al., Fermilab Technical Memo, FERMILAB-TM-2541-E (2012).

[28] M. Vesterinen, "An Improved Energy Calibration and Monte Carlo Over-smearing for Electromagnetic Clusters". D0 Note 6040 (2010).

[29] "Electron and Photon Identification with p20 data", O. Atramentov, D. Bandurin, X. Bu, B. Calpas, E. Carrera, D. Duggan, A. Ferapntov, M, Takahashi, T. Uzbyakova, H. Yin, D0Note 5761 (2008) and https://plone4.fnal.gov/P1/D0Wiki/objectid/emid/emdev/optEleID-2009/description_eleID.

[30] "Artificial Neural Network for Electron and Photon Identification", X. Bu, Y. Liu, D0Note 5545 (2007).

[31] “p20 ICR Electron Identification", J. Kraus, T. Gadfort, O. Atramentov, D0Note 5691

[32] "ICR Electron Efficiencies for Run IIa", B. Calpas, J. Kraus and T. Yasuda, D0Note 5939

[33] "ICR electron efficiencies for the Run IIb-1 and Run IIb-2 combined datasets", B. Calpas, J. Kraus and T. Yasuda, D0Note 6051

[34] "ICR Electron Identification with BDT/NN Combined Cut", X. Bu, L. Han, P. Jiang, Q. Li, D0Note 6271 
[35] "Certification of muon identification efficiencies and treatment of systematic uncertainties for the Run IIb dataset", Oleg Brandt, David Hedin, SungWoong Cho D0 Note 6326 and references therein.

[36] Improvements from the T42 Algorithm on calorimeter objects reconstruction, D0 Note 4335.

[37] "Updated measurement of the $Z Z$ Production Cross Section and Search for the Higgs in the Four Lepton final state using up to $9.9 \mathrm{fb}^{-1}$ of $p \bar{p}$ Collisions at $\sqrt{s}=1.96 \mathrm{TeV}$ " V. M. Abazov et al [D0 Collaboration], arXiv:physics/1304.5422.

[38] M. Petteni, G. Davies and T. Christoudias, Jet Energy Scale Determination at Do Run II, D0 Note 5382.

[39] "Measurement of the WZ $\rightarrow 111 \nu$ Cross Section and Limits on Anomalous Triple Gauge Couplings", A. Askew, K. Kaadze, J. Kraus, Y. Maravin, C. L. McGivern, D0 Note 6021.

[40] "Next-to-next-to-leading order soft gluon corrections in top quark hadroproduction", Kidonakis, N. and Vogt, R., Phys. Rev., D68,2003

[41] "Efficiency of the data quality calorimeter flags", Park, S. J. and Begel, M., D0 Note 5324.

[42] " $Z Z \rightarrow \ell \ell \nu \nu$ production in $p \bar{p}$ collisions at $\sqrt{(s)}=1.96 \mathrm{TeV}$ ", Abazov, V. M. et.al. The D0 Collaboration, D0 Note 5620-CONF.

[43] "Measurement of the cross section for $Z / \gamma * \rightarrow e^{+} e^{-} c$ production at D0", Fox, H. and others, D0 Note 5672. 
[44] Campbell, J. M. and Ellis, R. Keith, Phys. Rev. D, 60, 1999, hep-ph/9905386. http://mcfm.fnal.gov/. We use 6.2 with CTEQ6 pdf sets.

[45] W. Fisher, FERMILAB-TM-2386-E (2006).

[46] T. Junk, Nucl. Instrum. Methods Phys. Res. A 434, 435 (1999).

[47] A. Read, J. Phys. G 28, 2693 (2002). 
APPENDIX A

ELECTRON FAKERATES 
Here are shown the plots of the measured electron fakerates.
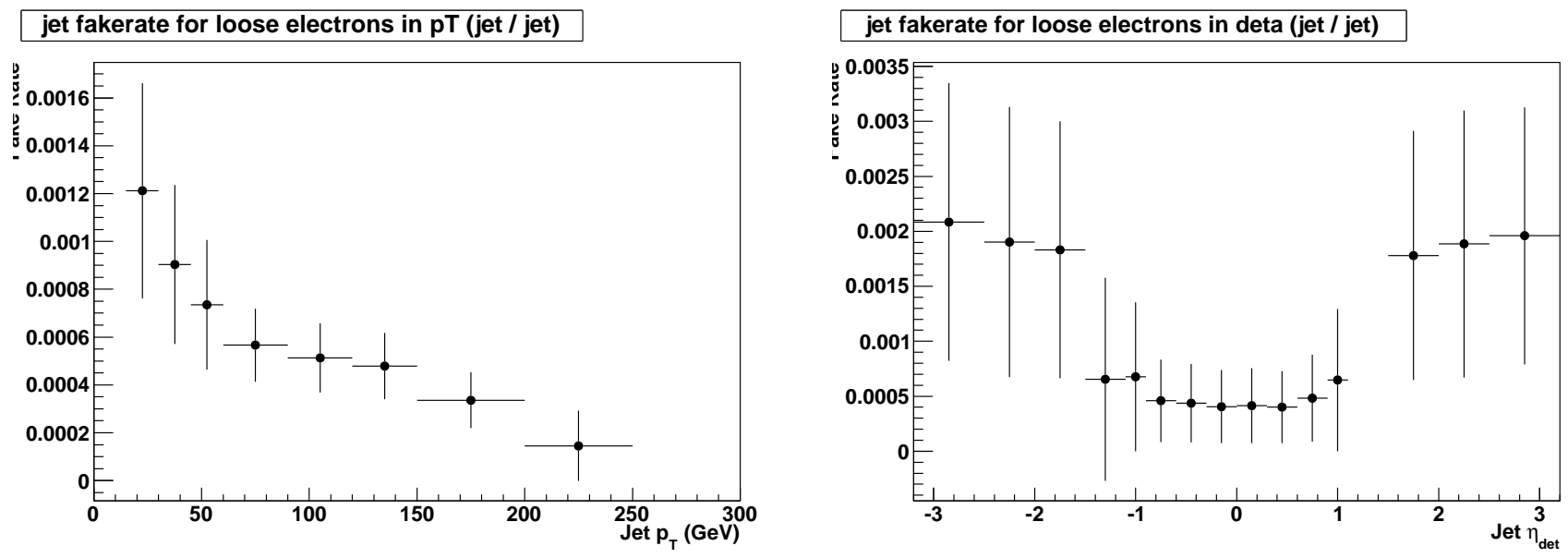

Figure A.1: RunIla: Plot of the measured electron fakerates. The left plot shows the fakerate as a function of jet $p_{T}$. The right plot shows shows the fakerate as a function of jet $\eta_{\text {det }}$. 

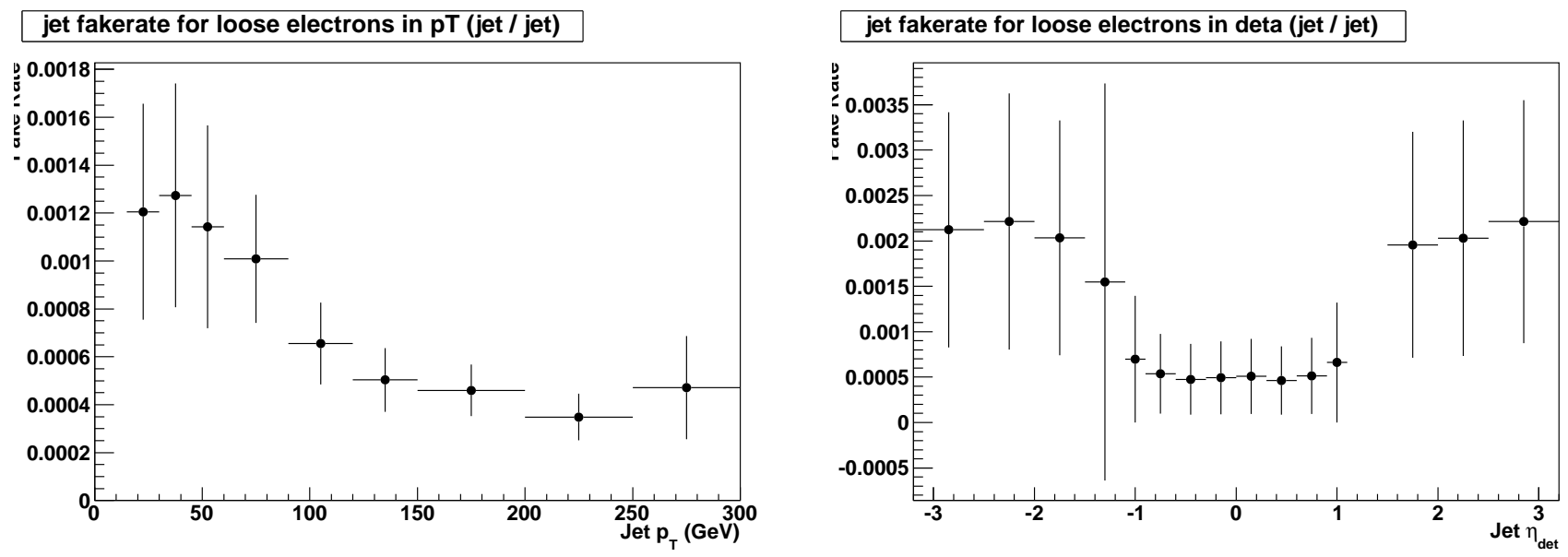

Figure A.2: RunIIb: Plot of the measured electron fakerates. The left plot shows the fakerate as a function of jet $p_{T}$. The right plot shows shows the fakerate as a function of jet $\eta_{\text {det }}$.

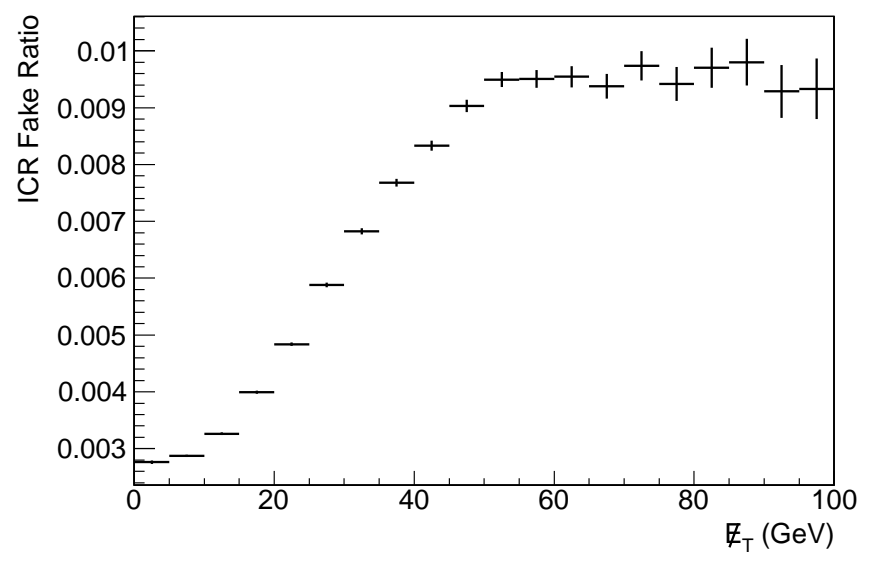

Figure A.3: Plot of the measured ICR electron fakerate vs. $\mathbb{E}_{T}$ 

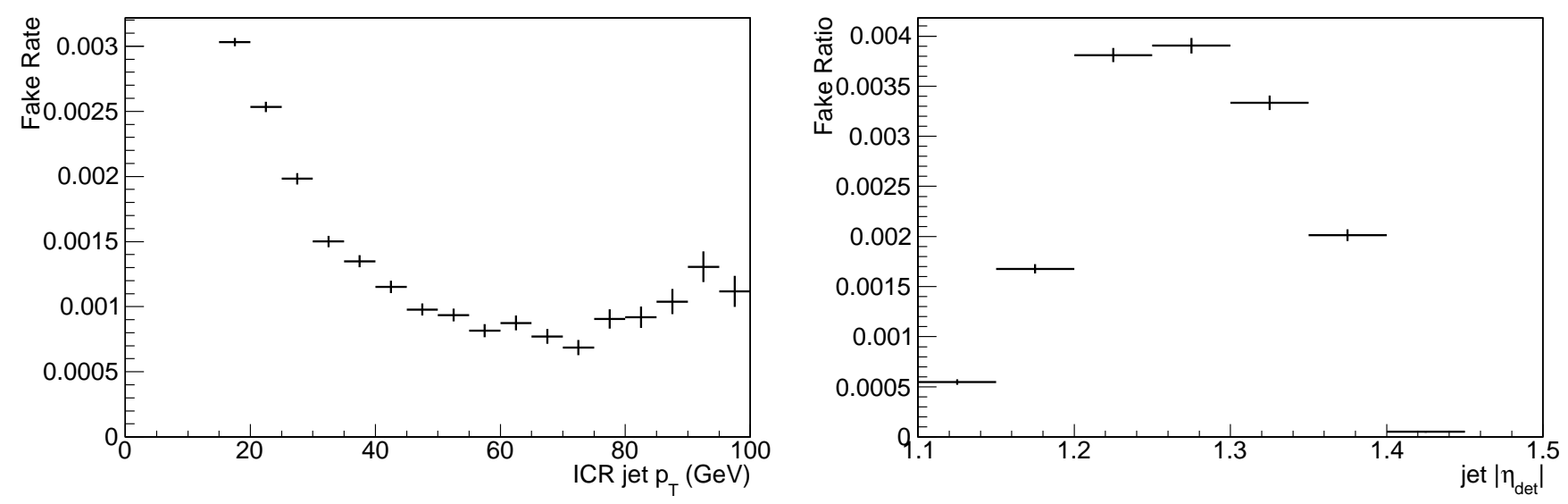

Figure A.4: RunIIa: Plot of the measured ICR electron fakerates. The left plot shows the fakerate as a function of jet $p_{T}$. The right plot shows shows the fakerate as a function of jet $\left|\eta_{\text {det }}\right|$.
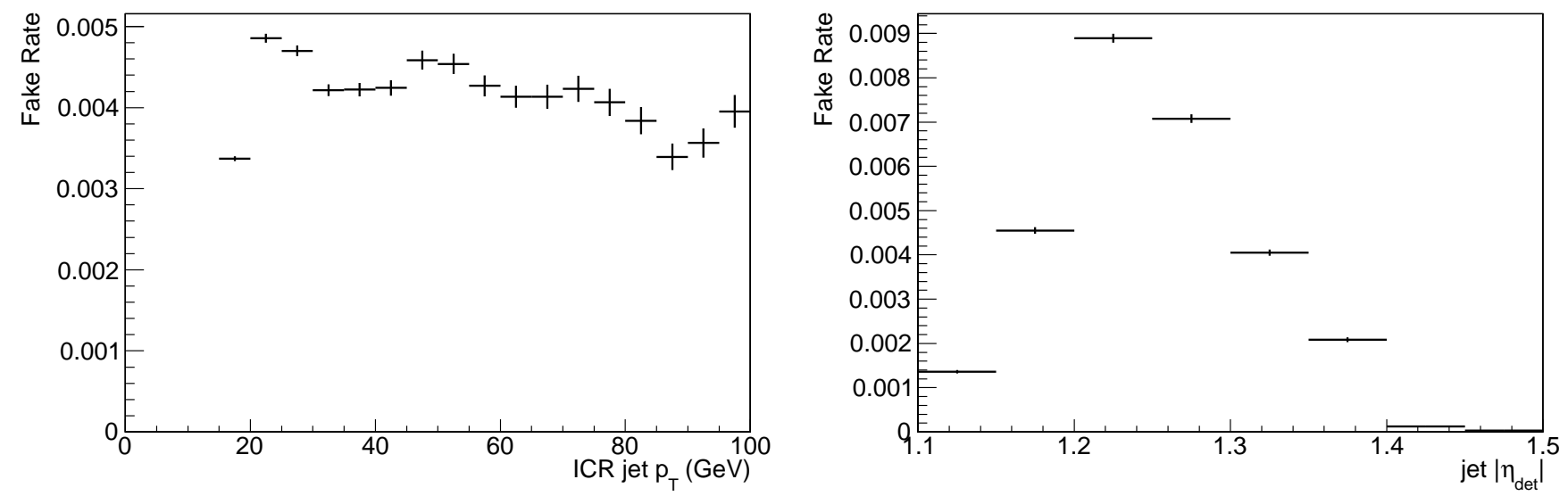

Figure A.5: RunIIb: Plot of the measured ICR electron fakerates. The left plot shows the fakerate as a function of jet $p_{T}$. The right plot shows shows the fakerate as a function of jet $\left|\eta_{\text {det }}\right|$. 
APPENDIX B

MUON FAKERATES 
Here are shown the plots of the measured muon fakerates.
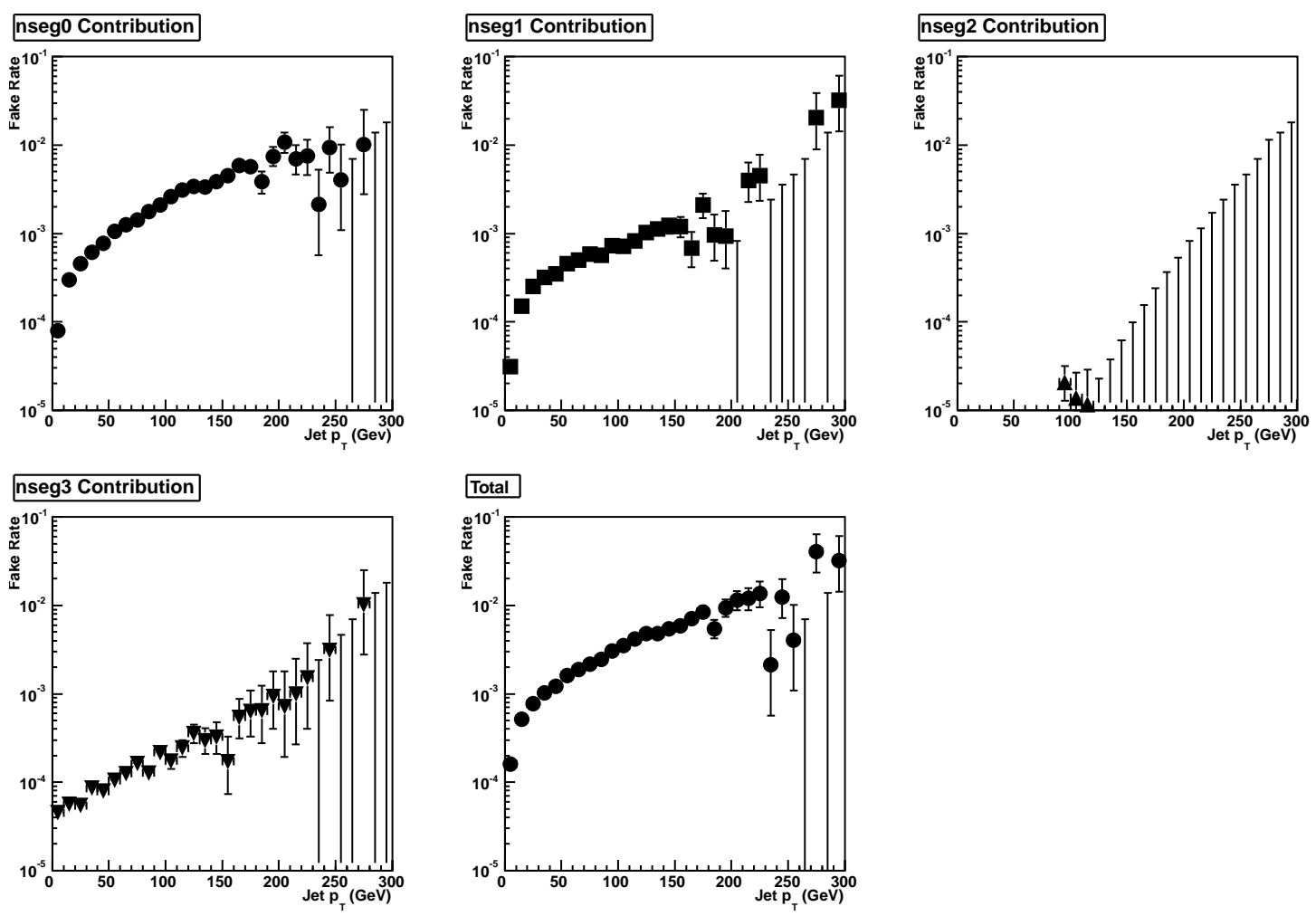

Figure B.1: RunIIa plots of the measured muon fakerates. The plots show the fakerate as a function of jet $p_{T}$ for muons with $p_{T}>15 \mathrm{GeV}$ when all muons are isolated for different values of nseg. 

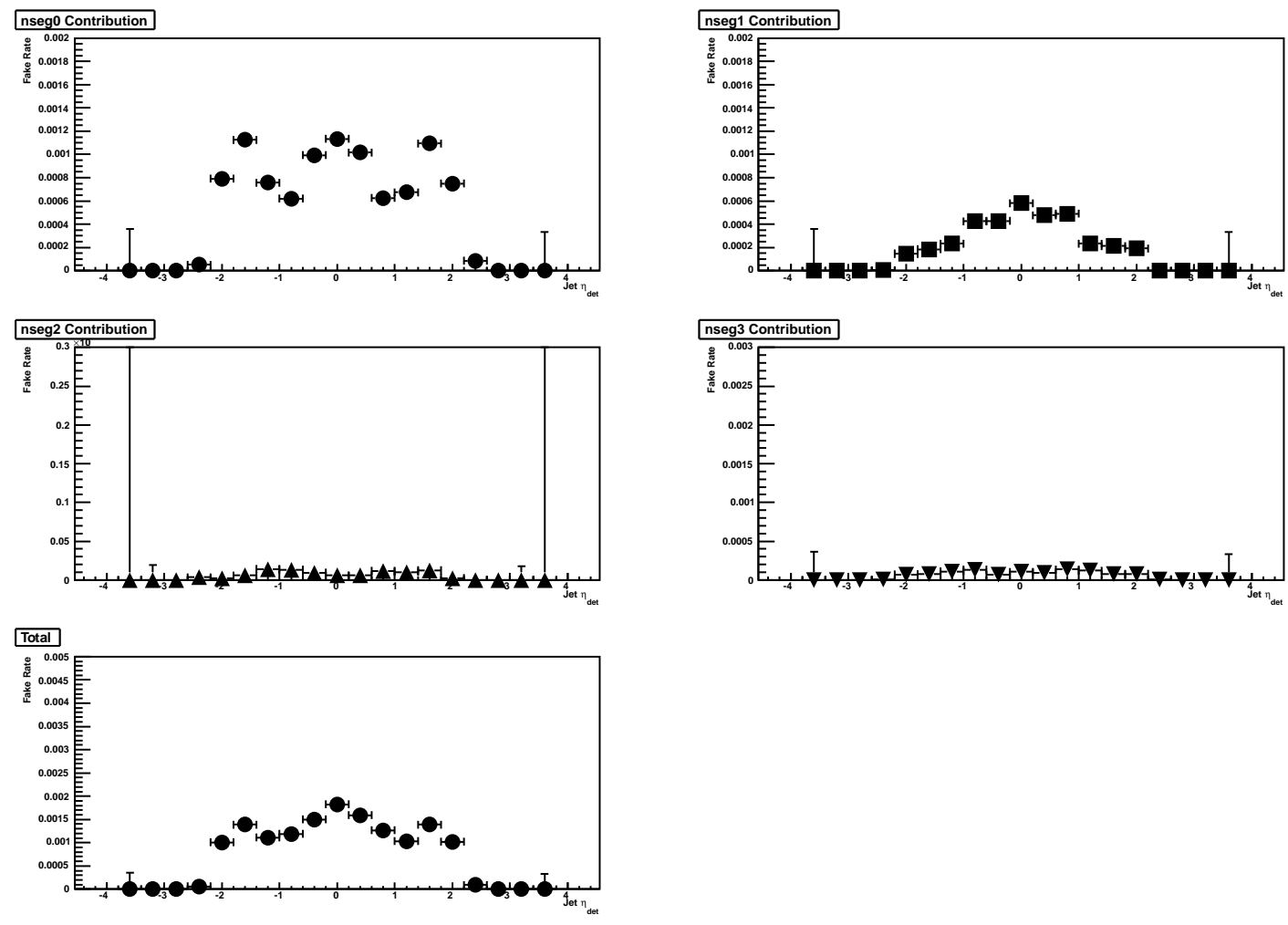

Figure B.2: RunIIa plots of the measured muon fakerates. The plots show the fakerate as a function of jet $\eta_{\text {det }}$ for muons with $p_{T}>15 \mathrm{GeV}$ when all muons are isolated for different values of nseg. 

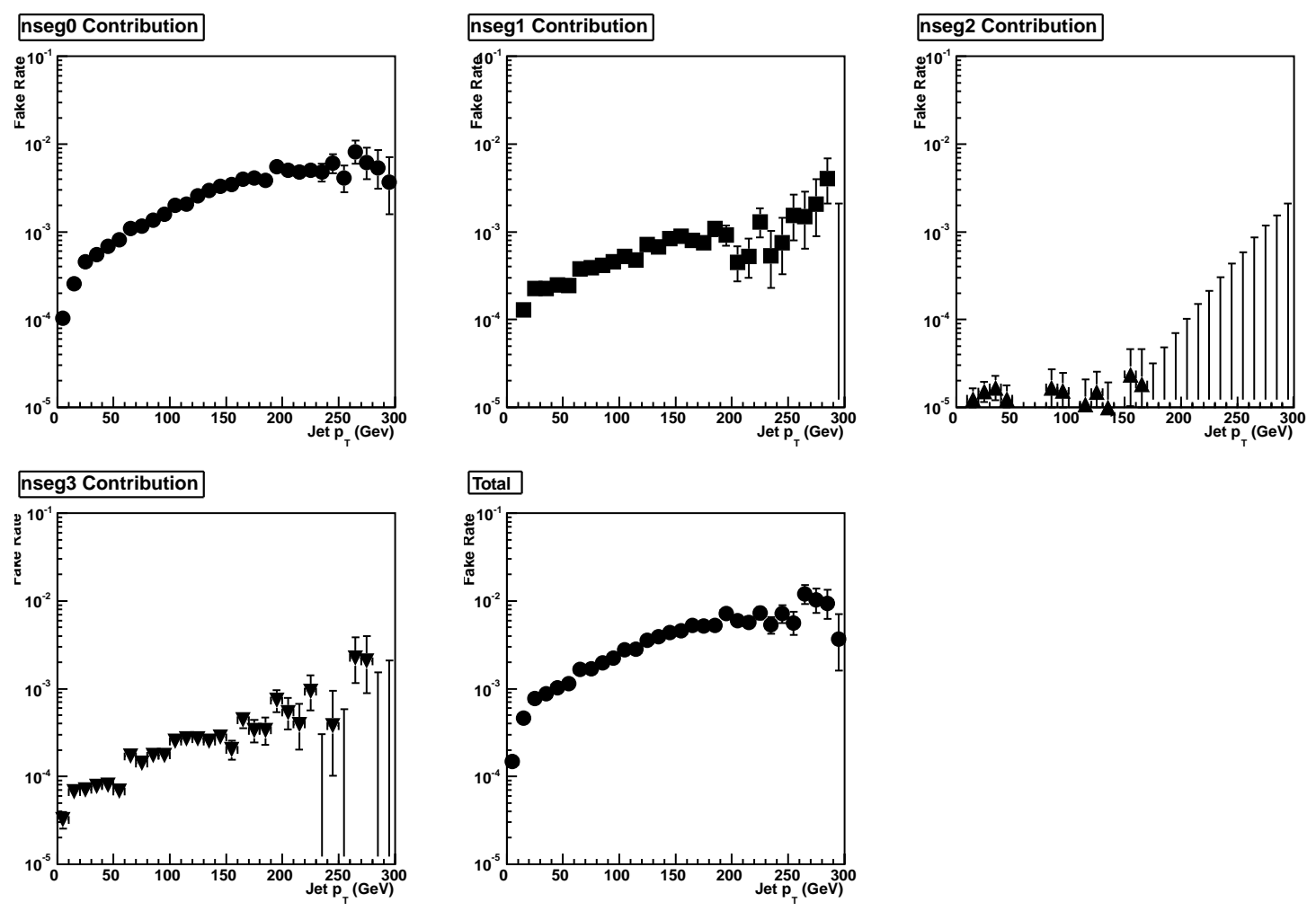

Figure B.3: RunIIb plots of the measured muon fakerates. The plots show the fakerate as a function of jet $p_{T}$ for muons with $p_{T}>15 \mathrm{GeV}$ when all muons are isolated for different values of nseg. 

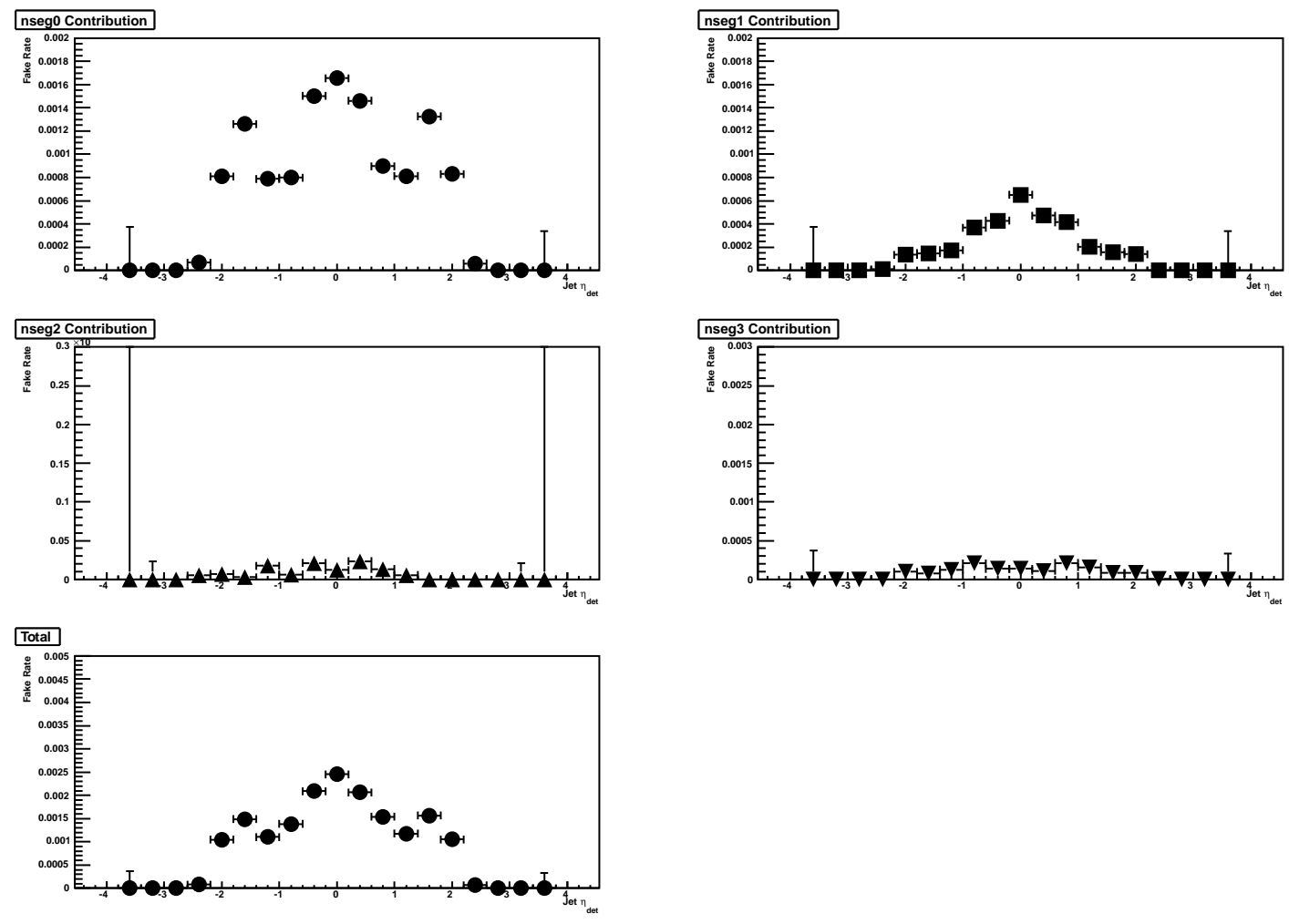

Figure B.4: RunIIb plots of the measured muon fakerates. The plots show the fakerate as a function of jet $\eta_{\text {det }}$ for muons with $p_{T}>15 \mathrm{GeV}$ when all muons are isolated for different values of nseg. 